\title{
Effects of Pristine and Nitrogen-Doped Multi-Walled Carbon Nanotubes on Human Lung Epithelial and Fibroblast Cells
}

Amy Mihalchik

Follow this and additional works at: https://researchrepository.wvu.edu/etd

\section{Recommended Citation}

Mihalchik, Amy, "Effects of Pristine and Nitrogen-Doped Multi-Walled Carbon Nanotubes on Human Lung Epithelial and Fibroblast Cells" (2016). Graduate Theses, Dissertations, and Problem Reports. 6230.

https://researchrepository.wvu.edu/etd/6230

This Dissertation is protected by copyright and/or related rights. It has been brought to you by the The Research Repository @ WVU with permission from the rights-holder(s). You are free to use this Dissertation in any way that is permitted by the copyright and related rights legislation that applies to your use. For other uses you must obtain permission from the rights-holder(s) directly, unless additional rights are indicated by a Creative Commons license in the record and/ or on the work itself. This Dissertation has been accepted for inclusion in WVU Graduate Theses, Dissertations, and Problem Reports collection by an authorized administrator of The Research Repository @ WVU.

For more information, please contact researchrepository@mail.wvu.edu. 
Effects of Pristine and Nitrogen-Doped Multi-Walled Carbon Nanotubes on Human Lung Epithelial and Fibroblast Cells

\author{
Amy Mihalchik \\ Dissertation submitted \\ to the School of Pharmacy \\ at West Virginia University
}

in partial fulfillment of the requirements for the degree of

Doctor in Philosophy in

Pharmaceutical and Pharmacological Sciences

Yong Qian, Ph.D., Advisor

Yon Rojanasakul, Ph.D., Chair

Vincent Castranova, Ph.D.

John Barnett, Ph.D.

Dale Porter, Ph.D.

Department of Pharmaceutical and Pharmacological Sciences

Morgantown, West Virginia

2016

Keywords: Multi-Walled Carbon Nanotubes, Nitrogen-Doped Multi-Walled

Carbon Nanotubes, Dosimetry, Fibrosis

Copyright 2016 Amy Mihalchik 


\title{
Abstract \\ Effects of Nitrogen-Doped and Pristine Multi-Walled Carbon Nanotubes in Human Lung Cells
}

\begin{abstract}
Amy Mihalchik
Multi-Walled Carbon Nanotubes (MWCNT) were first described by lijima in 1991 as "needle-like tubes" made up of concentric sheets of graphene lattice composed of hexagonal carbon units. MWCNT have been reported to be extremely strong, light-weight, and durable, thus making them a highly valued nanomaterial commercially. In order to improve their electrical conductivity and dispersibility for industrial and biomedical uses, materials scientists began functionalizing CNT in the mid-1990s. One type of functionalized MWCNT, nitrogen doped-MWCNT (ND-MWCNT), have nitrogen directly incorporated into the carbon lattice, disrupting $\mathrm{sp}^{2}$ bonding and decreasing the crystallinity of MWCNT. Nitrogen-doping significantly increases the brittleness, chemical reactivity, and n-type semiconductor activity of this material, and may also have interesting implications for its bioactivity. Alterations in physicochemical properties such as size, surface reactivity, agglomeration, and charge have been suggested to significantly impact the overall toxicity of MWCNT, but less in known on the effects of MWCNT functionalization. While the unique physicochemical properties of these materials generate exciting new possibilities for industrial and consumer products, the potential for unintended human exposure, especially and primarily through inhalation, has been of concern. Numerous animal studies have shown that MWCNT induce inflammation and pulmonary fibrosis at occupationally relevant exposure doses, but have yet to provide detailed information on the occupational risk of ND-MWCNT and other functionalized MWCNT. In recent years, the U.S. Environmental Protection Agency and National Toxicology Program have called for increased use and validation of predictive in vitro models to lessen the need for costly and time-consuming in vivo projects to determine general toxicity of nanomaterials. The studies presented here focused on developing and utilizing various in vitro models employing human Small Airway Epithelial Cells (SAEC), human bronchial epithelial cells (BEAS-2B), and normal human lung fibroblasts (WI-38) to study the bioactivities of ND-MWCNT and pristine Mitsui-7 MWCNT (MWCNT-7). However, it was found that the type of in vitro system utilized as well as the nanomaterial dosing schema could have serious and significant impact on the results and their subsequent interpretation and relevance to real-world exposures. Here, we provide an assessment of results on the inflammatory and fibrotic potential of ND-MWCNT and MWCNT-7 and the in vitro approaches we used to address these aims including monoculture and conditioned media, as well as concentration-based and surface area-based dosing. Results suggested that ND-MWCNT and MWCNT-7 induced an acute particle and dose-dependent inflammatory response in SAEC and BEAS-2B, suggesting that the physicochemical properties of these materials may impact their bioactivity. WI-38 directly exposed to ND-MWCNT and MWCNT-7 exhibited dose-dependent changes in collagen I production and gene expression of $\alpha$-SMA, which is suggestive of pro-fibrogenic signaling. WI-38 exposed to biologically relevant concentrations of epithelial-derived IL- 6 and IL- 8 in the absence of CNT exhibited more subtle responses than those directly exposed to CNT, but still demonstrates that multiple approaches may be used in vitro to study fibroblast responses. Overall, the work suggests that while in vitro approaches may be useful for assessing acute cellular responses, the ability to accurately predict the fibrogenic potential of MWCNT with different physicochemical properties has yet to be fully elucidated. However, the conclusions drawn from this work and critical analysis of current literature provides clear direction for the future of in vitro nanoparticle risk assessment.
\end{abstract}




\section{DEDICATION}

To my great-grandparents: You were all brave enough to put an ocean between you and your home countries in search of a better life for your descendants. You have succeeded and I will never be able to thank you enough. 


\section{ACKNOWLEDGEMENTS}

I would like to thank my advisor, Dr. Yong Qian, and each committee member, including Dr. Yon Rojanasakul, Dr. Vincent Castranova, Dr. Dale Porter, and Dr. John Barnett, for their time, advice, support, and knowledge throughout my graduate career. I am grateful for the experience of working with these scientists who have taught me so much.

I would also like to thank the people I worked with while at the Morgantown $\mathrm{NIOSH}$, especially those who inspired me with their commitment to occupational safety and health, and showed me a career path that can truly make a difference in the lives of those around me. I firmly believe that all people, regardless of educational level or economic status, have the right to work in an environment that allows them to maintain their health and ability to support themselves, their families, and their communities. Words cannot express how proud I am to have worked at a place committed to supporting this goal.

I would like to thank Dr. Weiqiang Ding, who was absolutely instrumental to characterizing my nitrogen-doped MWCNT, as well as Dr. Aleks Stefaniak for his assistance. Dr. Nikki Marshall, Dr. Donna Davidson, Dr. Bridget Hindman, Dr. Brandi Talkington, Dr. Jen Sisler, and Justine Shaffer provided insight on various techniques utilized in my work and constructive criticism that helped shape this project.

Most of all, I would like to thank my friends and family. The friends I have met here at WVU were some of the most interesting, quirky, and big-hearted people I will likely ever meet and I hope to continue these friendships long after school ends. My parents have been a constant source of support, love, and encouragement, and I would especially like to thank them for allowing me to explore, make messes, and always be curious about the world we live in since I was a little girl. To my husband TJ, I love you to the moon and back, and I cannot thank you enough for doing all the little things that allowed me the time and space to work towards this degree. You're my sounding board, my best friend, and if I do say so myself, the best spouse a girl could ever hope for. Last, but not least, I want to thank our dog Birch, who provided much comic relief and joy to the days I spent writing my dissertation, and like her mommy, is ALWAYS in need of a walk. We are, for sure, walking buddies for life. 


\section{Table of Contents}

Title...

Abstract

Dedication

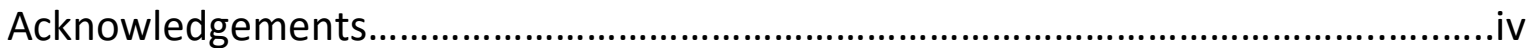

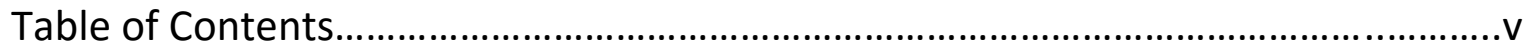

List of Figures and Tables....................................................................................

Abbreviations.........................................................................................................

Chapter 1

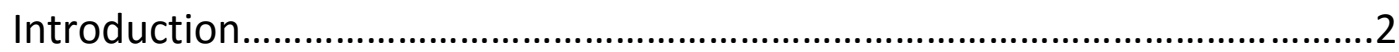

Alternative Testing Strategies in Nanotoxicology............................................

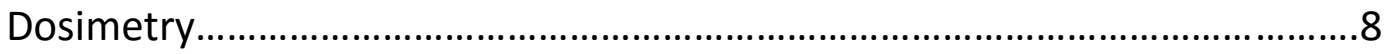

Multi-Walled Carbon Nanotubes..................................................................

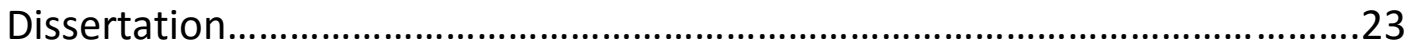

\section{Chapter 2}

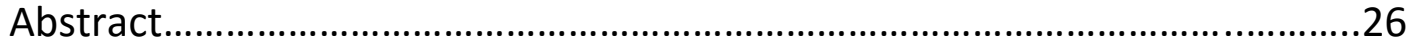

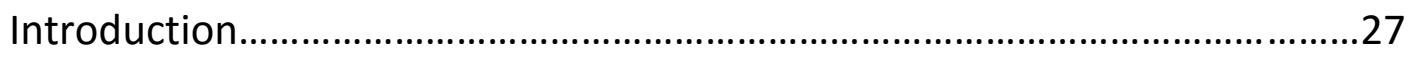

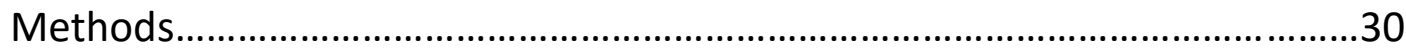

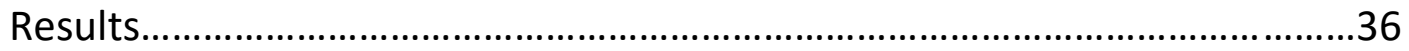

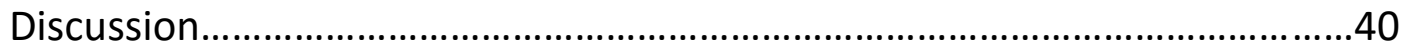

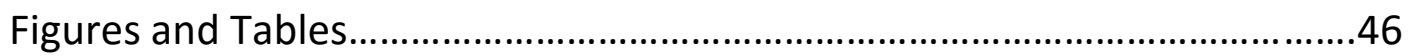

Chapter 3

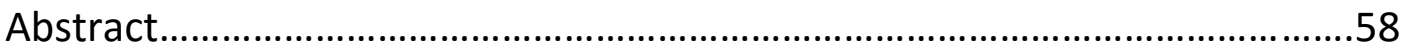

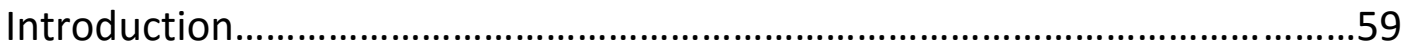

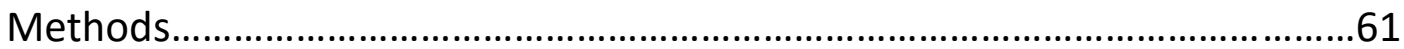

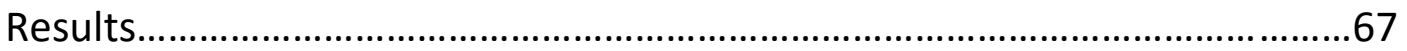

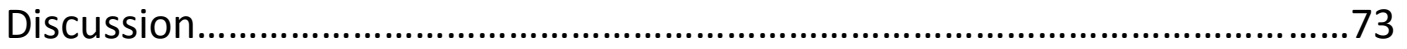

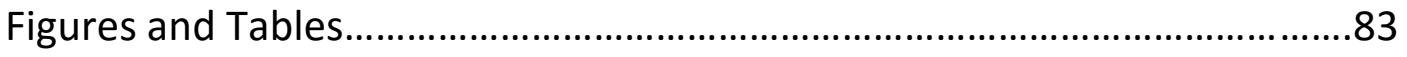

Chapter 4

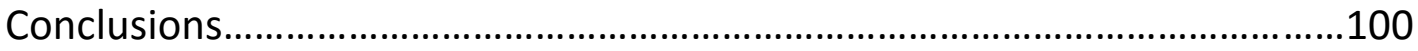

Appendix I

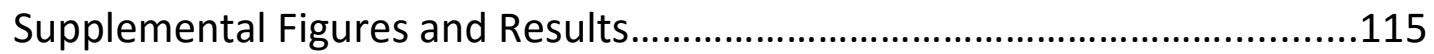

Supplemental Methods............................................................................134

Appendix II

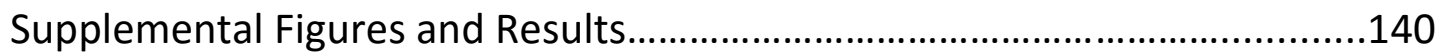

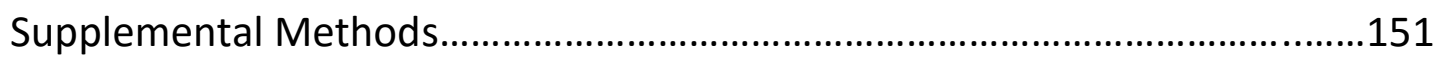

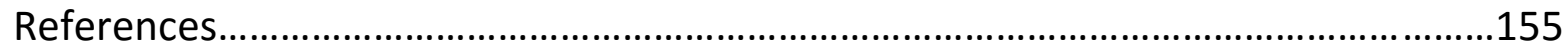

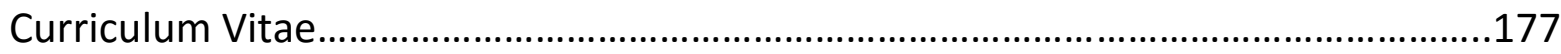




\section{Chapter 2}

\section{List of Figures and Tables}

\section{Figures:}

Figure 2.1: TEM and FESEM of ND-MWCNT.

Figure 2.2: XPS survey scan spectra of ND-MWCNT.

Figure 2.3: Raman spectra of ND-MWCNT and MWCNT-7.

Figure 2.4: XRD spectra of ND-MWCNT and MWCNT-7.

Figure 2.5: Cytotoxicity of ND-MWCNT and MWCNT over a dose range after $24 \mathrm{~h}$ exposure.

Figure 2.6: Uptake of $1.2 \mu \mathrm{g} / \mathrm{mL}$ ND-MWCNT and MWCNT-7 at 6 and $24 \mathrm{~h}$ in SAEC.

Figure 2.7: ND-MWCNT and MWCNT-7-induced ROS production in SAEC over 6 and $24 \mathrm{~h}$.

Figure 2.8: CDK4 western blot from SAEC exposed to ND-MWCNT or MWCNT-7 6 and $24 \mathrm{~h}$.

Figure 2.9. Total phospho-tyrosine and phospho-threonine western blots from SAEC exposed to NDMWCNT or MWCNT-7 6 and $24 \mathrm{~h}$.

Tables:

Table 2.1: Zeta potentials of ND-MWCNT and MWCNT-7

Table 2.2: SAEC cell cycle analysis after 6 and $24 \mathrm{~h}$ exposure to ND-MWCNT or MWCNT-7

\section{Chapter 3}

Figures:

Figure 3.1: Uptake of $1.25 \mu \mathrm{g} / \mathrm{cm}^{2}$ ND-MWCNT and MWCNT-7 at $24 \mathrm{~h}$ in BEAS-2B.

Figure 3.2: Uptake of $2.50 \mu \mathrm{g} / \mathrm{cm}^{2}$ ND-MWCNT and MWCNT-7 at $24 \mathrm{~h}$ in BEAS-2B.

Figure 3.3: Effect of ND-MWCNT and MWCNT-7 on BEAS-2B viability and proliferation.

Figure 3.4: ND-MWCNT and MWCNT-7-induced pro-inflammatory cytokines in BEAS-2B in a dosedependent manner. 
Figure 3.5: Experimental design to assess effects of biologically-relevant concentrations of IL-6 and IL-8 and direct exposure to ND-MWCNT or MWCNT-7 on WI-38 fibroblasts.

Figure 3.6: WI-38 cells engulf or are pierced by 0.125 to $2.50 \mu \mathrm{g} / \mathrm{cm}^{2}$ ND-MWCNT and MWCNT-7 after 48 h exposure.

Figure 3.7 Direct exposure of WI-38 to ND-MWCNT and MWCNT-7 alters cytotoxicity.

Figure 3.8 ND-MWCNT and MWCNT-7 interact with WI-38 at lower exposure dose $\left(0.0625 \mu \mathrm{g} / \mathrm{cm}^{2}\right)$ over $48 \mathrm{~h}$ exposure.

Figure 3.9 IL-6 and IL-8 induce limited effects on WI-38 viability and total cell counts but direct exposure reduces viability at high exposure doses.

Figure 3.10 Recombinant IL-6 and IL-8 and direct exposure to ND-MWCNT and MWCNT-7 significantly alter WI-38 proliferation assessed by CellTrace CFSE dye.

Figure 3.11 Recombinant IL-6 and IL-8 as well as direct exposure to ND-MWCNT and MWCNT-7 alter collagen I expression in WI-38 cells.

Figure 3.12 Induction of $\alpha$-SMA gene expression in WI-38 by ND-MWCNT and MWCNT-7.

Tables:

Table 3.1: P values associated with ND-MWCNT and MWCNT-7 induced cytotoxicity.

Table 3.2: P values associated with IL-6 and IL-8 induced changes in WI-38 proliferation

Table 3.3: $\mathrm{P}$ values associated with WI-38 proliferation after direct exposure to ND-MWCNT or MWCNT-

7.

Appendix I

Figures:

Supplemental Figure 1.1. SAEC expression of cell phenotype markers changes with culture conditions alone. 
Supplemental Figure 1.2. WI-38 expression of cell phenotype markers changes with culture conditions alone.

Supplemental Figure 1.3. CCL2 production is altered in SAEC and WI-38 under various co-culture conditions.

Supplemental Figure 1.4. Changes in dosimetry between monoculture and co-culture samples yield no significant changes in CCL2 production in SAEC and WI-38 within treatment groups.

Supplemental Figure 1.5. BEAS-2B in DMEM F-12 alter expression of cell phenotype markers changes with culture conditions alone.

Supplemental Figure 1.6. WI-38 in EMEM alter expression of cell phenotype markers changes with culture conditions alone.

Supplemental Figure 1.7. BEAS-2B in DMEM F-12 alter expression of cell phenotype markers changes with culture conditions alone.

Supplemental Figure 1.8. WI-38 in DMEM F-12 alter expression of cell phenotype markers changes with culture conditions alone.

Supplemental Figure 1.9. CCL2 production is altered in BEAS-2B and WI-38 under various culture conditions.

\section{Appendix II}

\section{Figures:}

Supplemental Figure 2.1. BEAS-2B proliferation is altered at high exposures to ND-MWCNT and MWCNT7.

Supplemental Figure 2.2. BEAS-2B engulf $4.8 \mu \mathrm{g} / \mathrm{mL}$ ND-MWCNT or MWCNT-7.

Supplemental Figure 2.3. BEAS-2B engulf $9.6 \mu \mathrm{g} / \mathrm{mL}$ ND-MWCNT or MWCNT-7.

Supplemental Figure 2.4. Experimental design for treating WI-38 with conditioned media. 
Supplemental Figure 2.5. WI-38 exposed to BEAS-2B-conditioned media show limited to no presence of ND-MWCNT or MWCNT-7 in TEM images.

Supplemental Figure 2.6. Direct exposure to ND-MWCNT or MWCNT-7 did not induce CCL2 production in BEAS-2B.

Supplemental Figure 2.7. BEAS-2B exposed to ND-MWCNT or MWCNT-7 increase gene expression of PDGF-A.

Supplemental Figure 2.8. BEAS-2B protein expression of PDGF-AA is not significant across concentrationbased and surface area-based exposures to ND-MWCNT or MWCNT-7.

Supplemental Figure 2.9. Changing doses did not lead to significant cell responses in WI-38. 


\section{Abbreviations}

\begin{tabular}{|c|c|}
\hline National Nanotechnology Initiative & NNI \\
\hline Reactive Oxygen Species & ROS \\
\hline National Toxicology Program & NTP \\
\hline Environmental Protection Agency & EPA \\
\hline Nation Institutes of Occupation Safety and Health & NIOSH \\
\hline National Research Council & NRC \\
\hline Food and Drug Administration & FDA \\
\hline Carbon Nanotube & CNT \\
\hline Multi-Walled Carbon Nanotubes & MWCNT \\
\hline Single-Walled Carbon Nanotubes & SWCNT \\
\hline Nitrogen-doped Multi-Walled Carbon Nanotubes & ND-MWCNT \\
\hline Mitsui-7 Multi-Walled Carbon Nanotubes & MWCNT-7 \\
\hline Enzyme Linked Immunosorbent Assay & ELISA \\
\hline In Vitro Sedimentation, Diffusion and Dosimetry & ISDD \\
\hline Mass Median Aerodynamic Diameter & MMAD \\
\hline Brunauer-Emmett-Teller & BET \\
\hline Chemical Vapor Discharge & CVD \\
\hline Dispersion Media & DM \\
\hline Air Liquid Interface & ALI \\
\hline Bronchoalveolar Lavage & BAL \\
\hline Lactate Dehydrogenase & LDH \\
\hline Field Emission Scanning Electron Microcopy & FESEM \\
\hline Tumor Necrosis Factor-alpha & TNF- $\alpha$ \\
\hline Interleukin-6 & IL-6 \\
\hline Interleukin-1-alpha & IL-1 $\alpha$ \\
\hline Interleukin-1-beta & IL-1 $\beta$ \\
\hline Transforming Growth Factor-beta & TGF $\beta$ \\
\hline Platelet-derived Growth Factor & PDGF \\
\hline Small Airway Epithelial Cells & SAEC \\
\hline C-C Chemokine Ligand 2 & CCL2 \\
\hline Vascular Endothelial Growth Factor-A & VEGFA \\
\hline Polymorphonuclear leukocytes & PMN \\
\hline Interleukin-8 & IL-8 \\
\hline Polyethylenimine & PEI \\
\hline Nod-like Receptor Family, Pryin Domain Containing-3 & NLRP3 \\
\hline Intercellular Adhesion Molecule 1 & ICAM-1 \\
\hline Vascular Cell Adhesion Molecule & VCAM-1 \\
\hline 1,2-dipalmitoyl-sn-glycero-3-phosphocholine & DPPC \\
\hline Phosphate Buffered Saline & PBS \\
\hline Double-distilled $\mathrm{H}_{2} \mathrm{O}$ & dd- $\mathrm{H}_{2} \mathrm{O}$ \\
\hline Transmission Electron Microscopy & TEM \\
\hline X-ray Photoelectron Spectroscopy & XPS \\
\hline
\end{tabular}


X-ray diffraction $\quad$ XRD

Dihydroethidium DHE

Sodium Dodecyl Sulfate-Polyacrylamide Gel Electrophoresis SDS-PAGE

Polyvinylidene Fluoride

PVDF

Tris Buffered Saline with Tween 20

TBS-T

Electron Spin Resonance Spectroscopy

ESR

Mitogen-activated Protein Kinase

MAPK

Phosphotidylinoside 3-kinase

PI3K

Idiopathic Pulmonary Fibrosis

IPF

Hydrogen Peroxide

$\mathrm{H}_{2} \mathrm{O}_{2}$

Recommended Exposure Limit

REL 
Chapter 1

Introduction 


\section{Introduction:}

Since Richard Feynman's talk in 1959, scientists and engineers have been designing new methods to create, manipulate, and utilize nano-sized materials [1]. According to the National Nanotechnology Initiative (NNI), a nanomaterial must have at least one dimension between 1 and 100 nanometers and can be made in a variety of structures for individual purposes (e.g. a particle for drug delivery purposes or a nanotube for products requiring tensile strength) [1]. It was expected that by 2015 , nanomaterials would be a \$1 trillion industry world-wide [2]. However, nanomaterials have unique characteristics that may pose a threat to workers and the public alike, necessitating further in-depth toxicological assessment [3]. As the field of nanotoxicology has rapidly expanded since its inception in 2005, researchers have been overwhelmed by the number of new products entering the market yearly, thus necessitating the identification of key parameters related to toxicity that can be applied across materials [4]. It has been determined that nanomaterial size, shape, surface area-to-volume ratio, reactive oxygen species (ROS) generating abilities, surface chemistry, and biopersistence all contribute to toxicity [3]. Additionally, it is important to note that "fine" and nano-sized materials of the same composition have different biological activities, suggesting that normally innocuous substances may became hazardous at the nanoscale.

As nanomaterials may be used for a wide variety of applications, government regulation has been spread across numerous agencies (e.g. NTP, EPA, NIOSH, FDA), and it has become clear to researchers across sectors that new methodology must be utilized to study nanomaterials. Unlike most chemicals or other soluble toxicants, nanomaterials require careful consideration of particokinetics to determine exposure doses within the model systems utilized. A strong collective push has been made in recent years to reduce reliance on in vivo models for toxicological testing in favor of high-throughput in vitro and in silico assays to create hazard rankings of materials based upon physicochemical properties, which the field has yet to fully adopt $[5,6]$. A dearth of validated and agreed upon in vitro models, 
assays, and dosimetry standards further complicate the ability of researchers and regulatory agencies to make this goal a reality. Although nanomaterials have the potential for wide-spread use in electronics, medical devices, industrial building materials, and environmental remediation [1], a major knowledge gap exists pertaining to the impact of nano-scale physicochemical properties and functionalizations on the study of toxicological mechanisms at the gross and cellular level of nanomaterials, especially nanofibers. One of the most widely studied nanofibers, multi-walled carbon nanotubes (MWCNTs), have been shown in vivo and in vitro to have inflammatory and fibrogenic potential. Little is known, however, on the relationship between the physicochemical properties and comparative toxicity of functionalized MWCNT and pristine MWCNT, despite the recent push in toxicological assessment of these particles. There are also methodological considerations that are of key importance. These issues, the comparative properties and toxicity of functionalized versus pristine MWCNT and the methodology of studying these particles, form the basis of this dissertation.

\section{Alternative Testing Strategies in Nanotoxicology:}

A wide variety of methods have been proposed to assess the general bioactivity, potential molecular mechanisms of action, and hazard ranking of nanomaterials. A primary issue surrounding nanotechnology is fear of the unknown. As new nanomaterials are synthesized, the backlog of untested materials also grows and the concerns of the public go unmet. The desire to be aware of potential toxicities before widespread use and human exposure has also increased, especially in light of public and occupational health disasters of the past century, such as the asbestos/mesothelioma connection. In 2005, the U.S. Environmental Protection Agency (EPA) and National Toxicology Program (NTP) jointly funded work by the National Research Council to generate a set of guidelines to direct the future of toxicology testing and address the immediate need for more efficient and reliable methods [6]. In 2007, the National Research Council published a bulletin with suggestions to increase reliance on in vitro methodologies to inform regulatory decision making while reducing the financial burden and time 
involved in risk assessment as compared to more time- and budget-consuming in vivo studies [7]. The bulletin also highlighted the need to place less reliance on animal testing due to potential idiosyncratic responses between test animals and humans, and push testing into advanced human in vitro models that may have more biological relevance. It stressed the importance of developing a tiered methodology utilizing relevant dosimetry between human exposures, animal, and human in vitro models to screen large numbers of toxicants and triage the most potent materials for further study in vivo to reduce the total number of animal studies required [8]. These suggestions are closely related to the " 3 R's" to Replace, Reduce, and Refine, a long-standing framework for humane scientific animal research proposed in 1959 [9]. With advances in genomics, in vitro methodology, and high-throughput screening research, it has nearly become within reach to begin applying the guidelines set forth to nanotoxicology $[6,10]$. Recently, Nel proposed the use and validation of robust, high-throughput human in vitro methods to study the new and unique hazards presented by nanomaterials to biological systems, and highlighted the great need for standardized assays [11]. Incremental progress has been made, and methods currently used for in vitro assessment are further described below.

Four primary methods have been utilized or proposed for in vitro nanotoxicology testing including monoculture, conditioned media approaches, co-culture, and three-dimensional culture. The paragraphs below provide brief sketches of the values and drawbacks of each system.

\section{Monoculture}

The use of monoculture assessment is the most basic in vitro model, featuring a single cell type cultured on a dish. This model is useful for quick assessment of a material, requiring the scientist to only select an appropriate cell line for the goals of the study and determine a dosing schema. There are a wide variety of primary and immortalized cell lines commercially available, and in some cases, cells can be directly collected from humans or rodents for further culture. The ease of monoculture assessment also allows for the potential use of multiple cell types side by side to confirm results and increase the 
robustness of the data. However, data from monoculture experiments may or may not be reflective of in vivo results or provide an accurate model of an in vivo exposure for various nanomaterials $[12,13]$. Monoculture systems alone also cannot address more complex research questions pertaining to cell-cell communication, the impact of tissue architecture (such as stretching of pulmonary tissues during inhalation and exhalation), or the roles of a multitude of other cell types on the in vivo response.

\section{Conditioned Media}

Two primary approaches fall within this category for nanotoxicology assessments. The first is to treat one cell type with the material of interest, collect media after exposure, centrifuge it to remove extraneous nanoparticles (if interested in only studying soluble cell mediators), and apply the conditioned media in empirically determined ratios to another cell line in order to address unidirectional cell-cell communication [14-16]. Although this method somewhat lessens the need to identify cell media-compatible cell lines compared to co-culture, it has limitations. The data from these systems may be more inconsistent experiment to experiment for several reasons. In order to achieve the same results, the nanomaterial exposed cells must produce a similar cytokine/chemokine protein response each time, the same amount of extraneous nanomaterial must be removed through centrifugation, and the proteins must be active and functional long enough to elicit a response in the cell line exposed to conditioned media, which also must have a similar response to previous experiments. Data analysis may be further complicated in the cell line receiving conditioned media for some assays, such as enzyme linked immunosorbent assays (ELISA) in which both cell types may produce the same protein (therefore necessitating additional experiments to determine if the nanomaterial exposed cell line is producing the signaling molecule of interest as well). It would also be difficult to study any potential autocrine mechanism in the cells exposed to conditioned media. A second alternative approach is to measure concentrations of cytokines and chemokines of interest in one cell type directly exposed to a nanomaterial and apply the same or similar concentration of commercially available recombinant 
protein to another cell type. However, this method has similar set-backs to the first approach. It would be extremely difficult to study the reverse effects of the second cell line on the first without using "twice" conditioned media, which could have real implications on the cellular responses and be just as interesting a relationship to study.

\section{Co-culture and Multi-culture}

Due to increased interest in the use of in vitro systems for regulatory decision making purposes, culture systems employing two or more cell lines have become de rigueur for analyzing the effects of cell-cell communication on a wide variety of endpoints. It is hoped that the use of these systems better reflect an in vivo response to a material from a molecular and genomic standpoint, which has been suggested by our lab and others $[17,18]$. Additionally, co-culture can be used to study bidirectional cell-cell communication between cell types which better recapitulates the in vivo setting and allows for a variety of research questions pertaining to cell behavior and responses to nanomaterials. These systems typically rely upon identifying two or more biologically relevant cell lines that would likely communicate in vivo, are compatible in culture, and maintain cell phenotypic activity. Proposed pulmonary culture systems have included alveolar epithelial-endothelial [19], epithelial-fibroblast [14, 18, 20-23], macrophage-epithelial [24, 25], and epithelial-macrophage-dendritic [26, 27], all of which ask and answer specific research questions. They also typically rely upon the use of removable Transwellß membranes or similar on which one or two cell types can be seeded, as well as another cell line in the basolateral chamber of the Transwell@ receiving dish. Transwell@ membrane composition can become a serious consideration, since not all cell lines will grow and form monolayers on the synthetic membranes available. The pore size (ranging from 0.4 to $8.0 \mu \mathrm{m}$ ) may allow the nanomaterial of interest to pass through the membrane into the basolateral chamber, which may be undesirable if one wishes to study the effects of soluble mediators only. Well-conceived experimental design is essential to success, such that cell seeding densities are reflective of in vivo, correct controls are added to 
compare between monoculture exposure, co-culture exposure, and each cell type alone within the coculture system, and that the correct dosimetry is utilized to ensure that the same exposure dose is given to monoculture and co-culture dishes. It is of importance to ensure that cell mediators can pass through the Transwell@ membrane, and that the cells under study are viable and also maintaining their phenotypes prior to nanomaterial exposure. Considering the scope and needs of each experiment, the cost alone can be prohibitively expensive, since a single Transwell@ plate costs more than ten times the cost of a standard six well dish. There will be increased need to prove that the questions these systems address are worth the cost now and in the future, especially if a less costly alternative could successfully triage nanomaterials for further study. The author has also observed a steady increase in very specific and novel systems to address pulmonary and other organ toxicology questions, and while admirable, there is an even stronger, yet unmet imperative for other laboratories to begin verifying these systems for reproducibility with the same and other nanomaterials [12, 19-23, 26, 28-30].

\section{Three-dimensional culture and advanced culture systems}

Although these systems are not a main focus of this dissertation, three-dimensional and other advanced culturing methods are growing in popularity as a system to model pulmonary disease and dysfunction [31-33]. These systems promote organotypic tissue organization and dynamic mechanical properties in 3-D models, but at the expense of easily assessing end points in each cell type alone [34]. One of the most interesting systems is an alveolar epithelial-endothelial model grown within a microdevice on each side of a flexible poly(dimethylsiloxane) membrane. The epithelial cells were maintained within AirLiquid Interface (ALI) conditions, and endothelial cells received a steady stream of circulating media and nutrients below. The microdevice also stretched to mimic normal inhalation and exhalation patterns, allowing for analysis of tissue barrier permeability and transport simultaneously. In order to assess functionality of the system, Huh et al. exposed the epithelial cells to aerosolized silica and found that the endothelial cells put under mechanical stretching increased expression of ICAM-1, which plays a role in 
vascular dysfunction, nearly 15 -fold compared to control and 3-fold more than stationary endothelial cells $[19,35]$. Despite promising results from these systems, it will remain important to further validate and ensure that the in vitro results are reflective of in vivo exposures. It also remains to be seen if the cost burden and lab equipment required to carry out experiments in advanced systems like these can be borne by enough labs to test a variety of materials.

\section{Dosimetry}

\section{Nanomaterial Dosimetry Models}

One of the most important, and historically most over-looked, considerations in nanotoxicology is dosimetry. While soluble chemicals and toxicants have traditionally been studied by concentration, nanomaterials require more careful reflection. In 2007, Teeguarden suggested, to little fanfare, a mathematical framework to delineate between the exposure dose and delivered dose of spherical nanoparticles, which takes diffusion, settling rate, and agglomeration of nanoparticles into account when determining the total deposited mass per cell or surface area of a culture dish [7]. Later called the In Vitro Sedimentation, Diffusion, and Dosimetry (ISDD) model, it was determined that not all spherical nanoparticles (polystyrene, silica, and iron oxide, in this study) exhibit the same behavior in cell culture media in vitro, and that the volume of media could significantly impact particle interactions with cells and cellular uptake [36]. It was also noted that changes in particle agglomeration and the composition of the protein corona, which is the protein coating found to form around nanoparticles, could impact particle delivery and uptake by cells [37]. DeLoid further suggested that nano-sized metal oxides with greater density and agglomerate diameter values were predicted by ISDD to deposit on the bottom of culture dishes quicker than smaller, less dense particles [38]. Together, these results suggest that the composition and characteristics of nanoparticles can decidedly alter the dose cells receive in vitro which could have interesting implications for nanotoxicology studies directly comparing two or more materials. It is important to note that models are still not available to distinguish between exposure and delivered 
doses of nanofibers such as MWCNT; hence, researchers are limited to normalizing nanofiber exposure dose between in vivo and in vitro studies at this point in time [37].

\section{Other Considerations in Nanomaterial Dosimetry}

In addition to potential confounding factors derived from particle behavior in vitro described above, there is a noticeable lack of consensus upon the definition of a "realistic" exposure dose for in vivo and in vitro studies. While in vivo studies are limited by a maximum dose an animal can withstand without dying during or shortly after aspiration, instillation, or inhalation, in vitro studies are far more open to interpretation and impractical exposure doses. For instance, many studies have used doses up to 100 to $200 \mu \mathrm{g} / \mathrm{mL}$ MWCNT, and even higher in some cases [39], which is of limited to no relevance to in vivo studies that utilized occupationally-relevant doses between 0.02 and $0.16 \mu \mathrm{g} / \mathrm{cm}^{2}$ [40-43]. To provide a clear example, the use of high doses have been justified by suggesting that the dose applied to a cell culture well with a small surface area (ranging between $0.32 \mathrm{~cm}^{2}$ to $55 \mathrm{~cm}^{2}$ for a 96 -well to $100 \mathrm{~mm}$ dish, respectively) could simply be extrapolated to the much larger mouse alveolar surface area as if the exact same dose was used as opposed to a true surface area-based dose [42]. Below is a visual representation of this concept:

Assuming the researcher would use a six well dish and apply $1.5 \mathrm{~mL}$ of media during the exposure

$\frac{100 \mu g}{m L} \times 1.5 m L=150 \mu g$

It can be projected that cells in vitro received the exposure dose below

$\frac{150 \mu g}{9.5 \mathrm{~cm}^{2}}=15.8 \frac{\mu g}{\mathrm{~cm}^{2}}$

And if considered in vivo, the exposure dose would be much lower based upon surface area.

$$
\frac{150 \mu g}{500 \mathrm{~cm}^{2}}=0.3 \frac{\mu g}{\mathrm{~cm}^{2}}
$$


Here, it is also important to note that altering the total amount of media used during exposure and the surface area of the dish could also impact the in vitro dose. While it is understandable to use a dose range that achieves the desired results in vitro, this method is perhaps not directly reflective of the in vivo state. A more relevant model would normalize the surface area dose between in vitro and in vivo using a standard ratio, such that the exposure doses were altered in tandem and remained constant based upon surface area regardless of model system, which was suggested at NIOSH (personal communication). Since in vitro methods have historically been used for molecular mechanism research instead of risk assessment, the selected dose range can also be crucial to understanding potential short and long-term risks of a material. Several years ago, Gangwal and Oberdörster separately suggested that occupationally-relevant in vitro dose ranges could be extrapolated from real-world human exposures that would span from an acute $24 \mathrm{~h}$ to working life-time exposure $[44,45]$. While the dissertation author has not come across a manuscript fully utilizing their suggestions for establishing such a dose range, it is certainly something to be considered from the standpoint of in vitro risk management.

\section{Current Methods of In Vitro Dosing}

Lack of standardization between using concentration, culture dish surface area, particle surface area, and particle number-based dosing between laboratories and studies has immensely complicated the ability of researchers to come to a consensus on how toxic a material is and which molecular mechanisms are involved, especially when exposure doses between in vivo and in vitro studies are often wildly different to achieve "significant" results in vitro. If in vitro systems are meant to be the future of regulatory testing, it is imperative that researchers understand dosimetry and the limitations of their model systems, and in turn, effectively communicate the methodology (including equations) and logic behind their selected doses in peer-reviewed published work. Below are explanations of common dosing methods and their limitations. 


\section{Surface Area Normalization for In Vitro Exposure}

Exposure doses for use in in vivo and in vitro pulmonary toxicology studies may be based upon potential human alveolar deposition values calculated from airborne concentrations collected during worksite assessments of labs and factories where MWCNT are produced [46, 47]. Alveolar deposition in a human can be calculated using the equation below, which accounts for exposure duration and the alveolar deposition rate based upon the mass median aerodynamic diameter (MMAD), a value which varies depending on the agglomeration and size of the particles and derived from studies using Micro-orifice Uniform Deposit Impactors [48]. Typically, particles with larger MMAD are predicted to deposit less in the alveolar region of the lung.

$$
\begin{aligned}
& \text { Human Alveolar Deposition }\left(\frac{\mu \mathrm{g}}{\text { time }}\right)=\text { Airborne concentration }\left(\frac{\mu \mathrm{g}}{\mathrm{m}^{3}}\right) \\
& \qquad \begin{array}{l}
\times \mathrm{E}\left(\frac{\mathrm{L}}{\mathrm{min}}\right) \\
\times \text { exposure duration }[\text { ex. }(8 \mathrm{~h} / \text { day }) \times(60 \mathrm{~min} / \mathrm{h})] \\
\times \text { alveolar deposition efficiency }(\%)
\end{array}
\end{aligned}
$$

Furthermore, the human deposition can be extrapolated to rodent deposition and in vitro cell culture dishes based upon the alveolar surface area and surface area of various cell culture dishes.

$\frac{\text { Human Deposition }}{\text { Human Alveolar Surface Area }}=\frac{\text { Mouse Deposition }}{\text { Mouse Alveolar Surface Area }}=\frac{\text { In Vitro Dose }}{\text { Culture Dish Surface Area }}$
A sample calculation for MWCNT based upon the work of Porter et al. for a six well culture dish is provided below [40]:

$$
\frac{226 \mu g M W C N T}{m^{2}}=\frac{\sim 10 \mu g M W C N T}{0.05 m^{2}}=\frac{0.19 \mu g M W C N T}{9.5 \times 10^{-4} m^{2}}
$$

It is important to make note of several caveats. 
1) Once calculated, the surface area based dose expressed in $\mu \mathrm{g} / \mathrm{cm}^{2}$ can easily be calculated for different cell culture dishes, thereby ensuring that each experiment, regardless of dish size, receives a more accurate exposure dose.

2) Nanoparticle settling has been considered in vitro studies, and therefore, the total administered dose in vitro may not reach the cells, which provides reasoning for why many scientists increase in vitro doses.

3) Computer-based particokinetic modeling programs specifically for fibers have yet to be fully developed, and much of what toxicologists have modeled about the delivered dose (as opposed to the exposure dose) have been based in spherical particles only. As discussed throughout, fibers have properties distinct from spherical particles that are not accounted for in these available models.

It is also important to note that surface area based dosing using this methodology was not originally a consideration in many in vitro nanotoxicology papers, and it is only within the past several years that such a paradigm has been considered more widely. Only this past year has this topic been considered at the annual Society of Toxicology conference. The use of concentration based $(\mu \mathrm{g} / \mathrm{mL})$ dosing, which historically originates from chemical toxicology work, is still widely published and used as a dosing paradigm by many laboratories. The concentration method is more accessible mathematically and straight-forward conceptually, which explains its continued prolific use.

\section{Particle Surface Area}

Unlike chemicals and many other materials previously studied by toxicologists, nanoparticles are unique in the strong inverse relationship observed between particle size and overall surface area. The smaller a nanomaterial is, the larger the surface area per unit mass when compared to fine sized or bulk counterparts. Additionally, the increased outer surface area of nanomaterials also increases the number 
and availability of reactive atoms or other moieties on the surface, which may increase their bioactivities [49]. Previous studies on a variety of materials including nanoscale $\mathrm{TiO}_{2}$ and ultrafine carbon black all suggested that the ultrafine materials induced greater toxic responses than fine sized materials when administered by mass [50-53]. Stoeger et al.'s study on six different ultrafine carbon based nanoparticles suggested that surface area normalization between materials best showed a doseresponse effect for pulmonary inflammation endpoints in mice [53]. However, it is difficult to accurately measure the surface area of fiber nanoparticles, and especially MWCNT, using standard characterization methods such as the Brunauer-Emmett-Teller (BET) gas adsorption method to derive specific surface areas of particles, though this technique has been previously used for spherical particles. The BET method can give varying results depending on if the MWCNT are agglomerated or single, and fully capped or open (which can occur during sonication or functionalization). Additionally, differences in the number of walls, diameter, and disruptions of the carbon lattice can all impact surface area [54]. Functionalized MWCNT and SWCNT have been shown to nearly double or triple in surface area measurements compared to untreated CNT, likely due to exposure of the inside of the tube, which can be inadvertently measured by BET if the tube becomes uncapped [54-56]. Methods to measure CNT surface area during occupational on-site worksite assessment are also lacking and may not accurately generate data on micrometer sized agglomerates of CNT typically found in the workplace, further eroding reliability of techniques available to determine CNT surface area [54,57]. Therefore, while particle surface area based exposure doses are interesting, it may be difficult to implement and ensure that reliable results are obtained from CNT sample to sample.

\section{Particle Number}

Particle number has also been considered as a potential parameter for nanotoxicology studies. However, the vast majority of research on this topic has pertained to spherical nanoparticles, which have been shown to behave differently from a toxicological standpoint compared to fibers. As of yet, 
computer-based particokinetic modeling has not been developed for fibers, making it a challenge to fully understand the behavior of these particles in standard in vitro systems. Fibers, especially MWCNT, have also been observed forming agglomerated bundles and multiple fibers often intertwine; these behaviors also change based upon the surface chemistry and charge of the MWCNT and further complicate counting true particle number per mass. Tools to quickly and accurately determine total fiber concentration in solution are also lacking. Nanoparticle Tracking Analysis, which measures light scattering and Brownian motion to compute the size distribution and concentration of nanoparticles in suspension, is based upon the assumption that the particle is a sphere within a size range that the length of most MWCNT fall outside of, and is therefore inaccurate for fibers. Newer technology found in the Malvern Archimedes instrument utilizing a micro Electro-Mechanical System sensor can determine the concentration and particle mass and size of large particles, but also may be limited in its ability to generate data for fibers.

\section{Multi-Walled Carbon Nanotubes}

\section{Overview of Multi-Walled Carbon Nanotubes}

MWCNT were reported as new carbon-based "needle-like tubes" made up of concentric sheets of graphene lattice composed of hexagonal carbon units by lijima in 1991 [58], although Oberlin likely synthesized and observed this nanomaterial first in 1976 [59]. MWCNT have been reported to be exceptionally strong and light-weight, with high electrical and thermal conductivity [60], thus making them a highly valued nanomaterial. The term MWCNT, however, is used to describe a wide variety of carbon structures composed of discrete concentric graphene tubes, the primary focus of this dissertation, as well as single graphene sheets rolled upon themselves like a scroll of paper. It is important to note that this encompassing term refers to MWCNT generally, without necessarily describing the length, diameter, wall number, purity, or surface chemistry, therefore increasing the importance of reporting characterization data in toxicological assessments. The specific MWCNT 
studied in this dissertation are Mitsui-7 pristine MWCNT, which have been well characterized and studied in a variety of contexts.

\section{Overview of Functionalized Multi-Walled Carbon Nanotubes}

Functionalized MWCNT were originally produced in order to enhance certain properties such as dispersibility, electrical conductivity, or ability to be functionalized again such that other groups could be added for use in biomedical purposes and other applications. Later, it became important that perhaps materials scientists could employ "safety by design" and purposefully synthesize CNT that have specific characteristics that make them less toxic in humans in the event that workers or consumers were exposed to these particles $[3,61]$. The phrase "functionalized MWCNT" is another catchall category in the literature, including MWCNT that have covalent attachment of chemical groups to the sides, tips, and defect sites (e.g. - $\mathrm{COOH},-\mathrm{NH}_{2}$ ) [62], noncovalent adsorption of surfactant molecules like polyethylene glycol or aromatic compounds $[62,63]$, doping with intercalated electron donors $(K, R b, N)$ or acceptors (F, B) [64], or attachment of siRNA or drug compounds [65]. It has been shown that the modification of MWCNT with various functionalizations has a significant impact on bioactivity in vivo and in vitro [66-71], but inconsistencies and gaps in the data exist which must be addressed. On a more positive note, the wide variety of functionalized MWCNT available for study give researchers an excellent opportunity to begin developing hazard rankings on which physicochemical properties alter MWCNT toxicity. The functionalized MWCNT primarily studied in this dissertation are nitrogen-doped MWCNT (ND-MWCNT), which have been shown to have increased surface defects and in vitro bioactivity compared to pristine Mitsui-7 MWCNT [72].

\section{Methods typically used to synthesize CNT}

Several primary methods are used to synthesize CNT which include arc discharge, laser ablation, and chemical vapor discharge (CVD). Briefly, the arc discharge method utilizes high temperatures and an electrical current to generate a large quantity of CNT from graphite electrodes in the presence of a 
metal catalyst (i.e. Ni or Fe). The ability to control CNT alignment is limited using this method, and follow-up purification to remove the metal catalyst is also required. The laser ablation method relies upon the use of high-powered lasers to vaporize graphite within a quartz tube. Metals tend to vaporize and are not incorporated into the CNT as much compared to other methods, however, this method is typically not capable of large scale CNT production and produces fewer uniform CNT. CVD, the most commonly used method for CNT synthesis, uses metal catalysts in the presence of gaseous hydrocarbon sources (such as ethylene or methane) to control the formation of CNT from the tip or base dependent upon the catalyst used $[73,74]$. This method is the most cost-effective method for large scale production of relatively pure CNT, and is also often used to generate functionalized CNT [74].

\section{Properties of MWCNT}

The $\mathrm{sp}^{2}$ bonds between the carbon atoms of a MWCNT have a higher tensile strength than those of steel or Kevlar, making them one of the strongest known materials [74]. Van der Waals forces act between the successive layers of a MWCNT, and free electrons present in the p orbitals of the $z$ axis are also present in the space between layers and therefore available for electrical conduction [75]. Depending on how the CNT are synthesized, the carbon lattice can be in a "zig-zag," "armchair," or chiral conformation, which provides information on theoretical electronic properties [75]. MWCNT can also be described using the Russian Doll model, in which concentric layers of tubes in increasing diameter compose the overall tube or the parchment model in which the graphene sheet is rolled upon itself. The multiple layers of MWCNT can shield the inner layers from chemical interactions assuming a cap is present on the end of the CNT and increase the tensile strength of the CNT [74]. This can also change their bioactivity, dependent upon the total number of reactive sites present on the surface of the fiber, which is closely related to the presence of surface defects and functional groups attached to MWCNT surface. 


\section{MWCNT Induced Bioactivity}

The study of MWCNT is multi-faceted and complex, drawing from and bridging nanotoxicology, fiber toxicology, inhalation toxicology, and materials science. MWCNT are extremely small and easily aerosolized; their proposed primary route of exposure is inhalation, especially in workplaces where they are synthesized. They truly are a unique family of nanoparticles that have been shown to induce a variety of biological responses including inflammation, pulmonary fibrosis, cancer, cardiovascular dysfunction, and potential teratogenic effects in vivo and in vitro $[40,57,72,76-87]$. When toxicologists first began studying MWCNT, their high aspect ratio and ability to settle in the alveolar region of the lungs were of primary concern. MWCNT were often compared to asbestos, arguably one of the most widely known public health concerns of the $20^{\text {th }}$ century, providing further support to determine their risks to human health $[11,88,89]$. As studies began to increase throughout the mid-2000's to present day, it became increasingly clear that the physicochemical characteristics of MWCNT played a key role in determining their overall toxicity.

\section{Pulmonary Effects of MWCNT-7}

Much of what is known on the occupational risk of MWCNT is based upon in vivo studies carried out within the past several years at NIOSH. These studies provided a conceptual framework that the author used to understand the issues surrounding MWCNT in relation to my dissertation work carried out at NIOSH. MWCNT-7, acquired from Mitsui \& Co., were determined to have 20-50 layers, with a mean width of $49 \pm 13.4 \mathrm{~nm}$ and mean length of $3.86 \mu \mathrm{m}$ indicative of a high aspect ratio also characteristic of crocidolite fibers $[40,90]$. In 2010, Porter et al carried out a time- and dose-dependent mouse study, treating groups with dispersion media (DM), which is reflective of bronchoalveolar lavage (BAL) fluid, or MWCNT-7 via single-dose aspiration at 10, 20, 40, or $80 \mu \mathrm{g}$; doses were derived from concentrations observed during a workplace assessment where CNT were synthesized [46]. BAL fluid was collected from mice at $1,7,40$, and 56 days post-exposure, and additional groups of mice were sacrificed to 
assess dispersion through pulmonary tissues, biopersistence, and fibrotic response of MWCNT [40].

From the same study, Mercer's group carried out MWCNT-distribution studies upon the fixed lungs with light microscopy and field emission scanning electron microscopy (FESEM) on slides stained with Sirius Red (to enhance contrast of MWCNTs and assess collagen deposition) or hematoxylin and eosin for pathology assessment [77]. Results suggested time and dose-dependent pulmonary damage and an acute inflammatory response marked by significant increases of lactate dehydrogenase (LDH) and albumin, as well as total polymorphnuclear (PMN) leukocytes in BAL fluid. Persistent granulomas were observed. FESEM images showed distribution of MWCNTs into alveolar epithelial cells and macrophages, appearing to "pierce" the cells, much like a needle [40, 77]. After 56 days, mice exposed to $80 \mu \mathrm{g}$ MWCNT-7 exhibited increased alveolar collagen deposition and tissue thickness, especially at sites of MWCNT deposition. In addition, MWCNT were able to enter the mesothelium, cross the endothelium into systemic circulation, and use macrophages to be ferried across the alevolar barrier [77]. Follow-up inhalation studies utilizing a different source and lot number of MWCNT-7 suggested similar experimental outcomes, and confirmed that nearly 65\% of MWCNT remained within the lung 336 days after exposure [78]. Several key comparisons were also made to the aspiration study: 1) although the aspiration lung burden was nearly 3 -fold higher than the inhalation burden, both induced the same level of increase in alveolar thickness, 2) 8 percent versus 21 percent of MWCNT were found in the alveolar interstitium post-aspiration or inhalation, respectively, and 3) less MWCNT agglomeration and accompanying granuloma formation was observed in the inhalation study $[40,77,78]$. These results likely differed due to route of administration; unlike aspiration, the inhalation exposure was extended over multiple days, allowing for more uniform and realistic deposition in the alveolar region while minimizing potential interaction between MWCNT at time of exposure [78]. Together, these data provided a frame of reference for understanding the impact of MWCNT at occupationally relevant doses. A more recent study by Dong et al. using the same MWCNT-7 as the aspiration study began to 
assess the molecular mechanisms elicited by aspiration exposures of 5,20 , and $40 \mu \mathrm{g}$ per mouse over a total of 14 days. Results suggested a dose-dependent induction of fibrogenic responses including: collagen deposition; increased expression of Col1a1, Col1a2, Fn1, and CCL-2 in alveolar tissue; acute inflammatory response measured by TNF- $\alpha$, IL-6, IL-1 $\alpha$, and IL-1 $\beta$ protein in BAL fluid; and timedependent changes in TGF $\beta$ and PDGF-AA, which are key to fibrogenesis [76].

\section{In Vitro Effects of MWCNT-7}

Several in vitro studies have also been completed using occupationally relevant doses of the MWCNT-7 described above. Snyder-Talkington worked extensively with these particles in Small Airway Epithelial Cells (SAEC) in monoculture and in an alveolar epithelial-endothelial co-culture system designed to recapitulate the alveolar-capillary interaction found in vivo. Monoculture results suggested that $24 \mathrm{~h}$ exposure to an occupationally relevant dose of MWCNT-7 induced ROS production, cell signaling activation through general phospho-tyrosine and phospho-threonine mediated pathways, and significant production of soluble CCL-2 and VEGFA [91]. The co-culture system, which utilized Transwells $\AA^{\circledR}$, showed that exposure to a low dose of MWCNT-7 increased the production of soluble cell signaling proteins including VEGFA, ICAM-1, and VCAM-1 which are related to inflammatory and angiogenic processes, and loss of endothelial barrier integrity concurrent with increased angiogenic behavior. Co-culture results also demonstrated increased global gene expression in accordance with in vivo gene expression, especially in endothelial cells, which suggested that the co-culture system may be more predictive of in vivo results. Wang et al. utilized an alternative approach to Snyder-Talkington, and instead treated SAEC with $0.02 \mu \mathrm{g} / \mathrm{cm}^{2} \mathrm{MWCNT}-7$ (same as $10 \mu \mathrm{g}$ dose in vivo in mice) continuously over 6 months. Results suggested that chronic exposure to MWCNT-7 increased the proliferative rate of SAEC compared to passage matched controls, invasive and migratory behaviors, and proto-oncogene signaling [92]. 


\section{Physicochemical Characteristics Impact MWCNT-Induced Bioactivity}

Physicochemical characteristics refer to the size, shape, agglomeration, surface area, charge, presence of defects, functionalization, and chemical composition, amongst other additional traits, of a material. Understanding these characteristics allows researchers to better recognize which ones appear to drive toxicity, allowing for "safety by design" to strategically produce MWCNT that should be less bioactive and harmful in humans. Below are brief discussions of select physicochemical characteristics and their impact on MWCNT-induced toxicity.

\section{Shape and Length}

Jia et al. compared the toxicity of carbon nanomaterials including SWCNT, MWCNT, and spherical $\mathrm{C}_{60}$ fullerenes in guinea pig alveolar macrophages and showed that SWCNT $>$ MWCNT $>C_{60}$ when assessed for cytotoxicity across a range of doses. The $\mathrm{C}_{60}$ showed nearly no toxic response, suggesting that the shape of a nanomaterial can significantly impact toxicity, even when composed of the same base material [93]. The length of MWCNT has also been shown to impact toxicity, such that longer MWCNT were typically more toxic than shorter MWCNT. To determine if MWCNT behaved similarly to asbestos in the mesothelium, Poland et al. injected long and short MWCNT samples into the peritoneal cavity of mice. Results showed that long, but not short, MWCNT induced acute PMN and protein influx into the peritoneal cavity, followed by production of granulomatous lesions [89]. Furthermore, Murphy et al. demonstrated length-dependent inflammatory and fibrotic responses over a 24-week period in mice after injection of long and short MWCNT directly into the pleural space. The authors concluded that perhaps long fibers were trapped and retained in the pleural space and unable to exit in the flow of pleural fluid, unlike short MWCNT that could negotiate through stomata and enter lymphatic circulation [94]. A follow-up in vitro study using the same MWCNT panel showed that THP-1 macrophages exposed to long MWCNT released IL-1ß, IL-6, and IL-8, and exhibited signs of "frustrated phagocytosis," meaning that the macrophages were unable to fully engulf the long MWCNT, prolonging the inflammatory 
response [95]. Together, the results argued that a two-pronged mechanism involving long MWCNT retention and prolonged macrophage activation could induce problematic pulmonary issues [94, 95].

\section{Agglomeration}

The agglomeration state of MWCNT can impact the overall response and toxicity observed in in vivo and in vitro studies, and is an important consideration if attempting to address occupational exposures [57]. Li et al. showed that agglomerated MWCNT delivered by intratracheal instillation in mice induced bronchial inflammation and severe destruction of the alveoli after 24 days, while concurrent inhalation studies (which delivered particles primarily in the smaller-sized respirable fraction) induced less of a negative pulmonary response, suggesting that deposition of large agglomerates, similar to long MWCNT, are perhaps less likely to break down and be cleared from the lung. Pauluhn's study of rats exposed to nose-only inhalation of MWCNT (from Baytubes) found that agglomerates did not disaggregate into individual nanotubes and exhibited less pulmonary clearance after 6 months, but did not appear to induce severe fibrosis [96]. In an aspiration study in mice using Mitsui-7, Mercer et al. observed granulomatous inflammation walling off areas with MWCNT agglomerates in alveolar airspace, while single or small diameter MWCNT bundles induced collagen deposition and thickening of the alveolar wall after 56 days [77]. A follow-up long-term inhalation study in mice showed that smaller, nonagglomerated MWCNT induced fibrotic responses and agglomerated MWCNT were either cleared or disaggregated over 168 days, unlike Pauluhn's study over a similar time span suggesting that physicochemical characteristics of MWCNT could impact the agglomeration/disaggregation of MWCNT in vivo. With consideration to all data presented above, it appears that agglomerated MWCNT are more likely to induce a persistent inflammatory response, while disaggregated MWCNT are more likely to produce a fibrotic response. 


\section{Surface Defects}

Surface defects may result from mechanical abrasion, incorporation of heteroatoms into the carbon lattice, acid-washing, and synthesis temperature $[97,98]$. Fenoglio et al. showed that grinding pristine MWCNT generated defects through disruption of the carbon lattice that increased dangling bonds, significantly increased the hydrophilicity, and scavenged external ROS better than pristine MWCNT [99]. The companion paper which assessed the toxicity of the same MWCNTs in Wistar rats, however, showed that the ground MWCNT increased LDH activity, total protein content, the number of macrophage and neutrophils, and granuloma formation in the rat lungs compared to those treated with heat-treated MWCNT (which reduced defects). Ground MWCNT also increased IL-1 $\beta$ and TNF- $\alpha$ in BAL fluid, therefore suggesting that the presence of surface defects and dangling bonds could indeed induce more severe toxicity regardless of ability to quench ROS in an acellular system. This indicates that ROS generation/scavenging is not, perhaps, the best framework to predict MWCNT toxicity as suggested by Tsuruoka $[100,101]$.

\section{Functionalization}

Although functionalized carbon nanotubes have been in production since the early 2000 's, not as much is known on their toxicity and impact on biological systems compared to pristine MWCNT. Li et al. carried out a concurrent in vivo/in vitro study assessing MWCNT functionalized with - $\mathrm{COOH}, \mathrm{PEG},-\mathrm{NH}_{2}$, sidewall $-\mathrm{NH}_{2}$, and $\mathrm{PEI}$; results suggested that surface charge may impact fibrogenic potential, with the most positively charged, PEI-MWCNT, eliciting the greatest toxicity in vitro and in vivo, and anionic MWCNT-COOH conversely eliciting the least toxicity [68]. Several studies corroborated that carboxylation of MWCNT, which greatly improved hydrophilicity, reduced bioactivity in vivo and in vitro, and also reduced NLRP3 inflammasome activation [71, 102, 103]. Tabet et al. also showed that coating MWCNT with a polystyerene-based polymer significantly reduced in vivo and in vitro bioactivity, suggesting that it is possible to design and functionalize MWCNT to be less toxic [104]. The effects of 
nitrogen-doping on bioactivity are extensively discussed in the introductory material of Chapter 2 , and therefore not discussed here.

\section{Specific Research Objective}

The purpose of this study was to better understand the role of physicochemical properties on the bioactivity of ND-MWCNT and pristine MWCNT through the use of multiple human lung cell in vitro models.

Aim 1: Observe the effects of ND-MWCNT and MWCNT-7 on inflammatory and fibrogenic processes in human lung in vitro models

Aim 2: Examine the role of in vitro dosimetry and model system in the study of ND-MWCNT and MWCNT-7

\section{Conceptual Outline of Dissertation}

\section{Chapter 2: Assess effects of nitrogen-doped multi-walled carbon nanotubes compared to pristine} multi-walled carbon nanotubes on human small airway epithelial cells

ND-MWCNT are functionalized MWCNT with enhanced electrical conductivity, hydrophilicity, and surface reactivity for further functionalization steps. In order to address the impact of physicochemical properties on bioactivity, ND-MWCNT were first characterized to determine size, structure, and composition as compared to pristine MWCNT-7, followed by analysis in Human Small Airway Epithelial Cells at occupationally relevant doses to assess ROS production, cellular proliferation, cell cycle distribution, and general cell signaling pathways.

Chapter 3: Assess the impact of direct and indirect exposure to ND-MWCNT and MWCNT-7 on human lung fibroblasts

It has been established that MWCNT can pass through the epithelium of the lung and into the interstitial space where they may interact with resident fibroblasts as well as translocate into systemic circulation [79]. In order to determine the effects of direct exposure of ND-MWCNT and MWCNT-7 on WI-38, a 
series of dose response assays were carried out to assess cytotoxicity, proliferation, collagen 1

production, and expression of genes related to myofibroblast activation and fibrosis. Additionally, it has been previously established that relationship between the epithelial cells and fibroblasts is one of great importance to understanding the fibrotic potential of MWCNT as epithelial cells are the second most likely cell type to be penetrated and provide the barrier into the interstitial space [105]. In order to study this relationship, a model of human bronchial epithelial cells (Beas-2B) and WI-38 was conceived to determine the impact of ND-MWCNT and MWCNT-7 induced epithelial-derived interleukin 6 (IL-6) and interleukin 8 (IL-8) on fibroblast proliferation and collagen 1 production.

\section{Chapter 4: Conclusions and Future Directions}

General conclusions on the body of work will be discussed here as well as future directions to carry the project forward.

\section{Supplemental Data}

Despite the desire of regulatory agencies to transfer the bulk of toxicology risk assessment to in vitro models, these systems have shown a wide variety of responses that yield both false positives and false negatives. This necessitates further study of how toxicologists carry them out and generate meaningful data predictive of in vitro exposures. Many attempts were personally made to create an epithelial:fibroblast co-culture system designed to study the fibrogenic potential of MWCNT; the various iterations of this system provided both an excellent learning opportunity and future starting point for other scientists. A conditioned media approach utilizing epithelial cells and fibroblasts was also attempted. There were many considerations put to question when developing these systems and analyzing the systems of others, namely: 1) cell types utilized, 2) growing and exposure conditions, 3) changes in cell phenotype during the experiment, 4) appropriate controls, and 5) physiological relevance. 


\section{Chapter 2}

\section{Effects of nitrogen-doped multi-walled carbon nanotubes compared to pristine multi-walled carbon nanotubes on human small airway epithelial cells}

Citation: Mihalchik, A.L., Ding, W., Porter, D. W., McLoughlin, C., Schwegler-Berry, D., Sisler, J., Stefaniak, A. B., Snyder-Talkington, B., Cruz-Silva, R., Terrones, M., Tsuruoka, S., Endo, M., Castranova, V., and Qian, Y. Effects of nitrogen-doped multi-walled carbon nanotubes compared to pristine multi-walled carbon nanotubes on human small airway epithelial cells. Toxicology, 2015. (333):25-36.

doi:10.1016/j.tox.2015.03.008

Adapted from Toxicology, 2015, (333):25-36 


\begin{abstract}
Nitrogen-doped multi-walled carbon nanotubes (ND-MWCNT) are modified multi-walled carbon nanotubes (MWCNT) with enhanced electrical properties that are used in a variety of applications, including fuel cells and sensors; however, the mode of toxic action of ND-MWCNT has yet to be fully elucidated. In the present study, we compared the interaction of ND-MWCNT or pristine MWCNT-7 with human small airway epithelial cells (SAEC) and evaluated their subsequent bioactive effects. Transmission electron microscopy, X-ray photoelectron spectroscopy, Raman spectroscopy, and X-ray diffraction suggested the presence of $\mathrm{N}$-containing defects in the lattice of the nanotube. The NDMWCNTs were determined to be $93.3 \%$ carbon, $3.8 \%$ oxygen, and $2.9 \%$ nitrogen. A dose-response cell proliferation assay showed that low doses of ND-MWCNT $(1.2 \mu \mathrm{g} / \mathrm{ml})$ or MWCNT-7 $(0.12 \mu \mathrm{g} / \mathrm{ml})$ increased cellular proliferation, while the highest dose of $120 \mu \mathrm{g} / \mathrm{ml}$ of either material decreased proliferation. ND-MWCNT and MWCNT-7 appeared to interact with SAEC at $6 \mathrm{~h}$ and were internalized by $24 \mathrm{~h}$. ROS were elevated at 6 and $24 \mathrm{~h}$ in ND-MWCNT exposed cells, but only at $6 \mathrm{~h}$ in MWCNT-7 exposed cells. Significant alterations to the cell cycle were observed in SAEC exposed to either $1.2 \mu \mathrm{g} / \mathrm{ml}$ of ND-MWCNT or MWCNT-7 in a time and material-dependent manner, possibly suggesting potential damage or alterations to cell cycle machinery. Our results indicate that ND-MWCNT induce effects in SAEC over a time and dose-related manner which differ from MWCNT-7. Therefore, the physicochemical characteristics of the materials appear to alter their biological effects.

Keywords: Multi-walled carbon nanotubes, functionalized multi-walled carbon nanotubes, reactive oxygen species
\end{abstract}




\section{Introduction}

As the field of nanotoxicology rapidly expands, researchers are working towards the identification of key toxicity parameters that can be applied across materials. Since their introduction, MWCNT have been used in a wide variety of industrial applications due to their unique physicochemical properties; however, these unique characteristics may pose a threat to workers and the public during production, use, and disposal $[59,106]$. The high aspect ratio, surface characteristics, durability, and redox potential of MWCNT contribute to their bioactivity, therefore suggesting potential routes for improving their safety profile [106]. Pristine MWCNT (including MWCNT-7) have been shown in numerous studies to induce acute inflammatory and chronic fibrogenic responses both in vitro and in vivo $[40,77,83,91,107]$. In order to improve MWCNT safety and biocompatibility, material scientists have begun implementing "safety by design" practices in the synthesis of carbon nanotubes (CNT) with alternative physicochemical properties. With this in mind, next-generation MWCNT may be more or less toxic compared to their first generation counterparts and may cause toxicity through alternative mechanisms.

MWCNT-7 have been shown to induce inflammation and fibrosis in vivo at occupationally relevant doses in mice and rats $[40,78,80,105]$. At an inhalation exposure of $5 \mathrm{mg} / \mathrm{m}^{3}, \mathrm{MWCNT}-7$ were capable of bypassing the defenses of the mouse lung due to their small size, with approximately $84 \%$ of the MWCNT-7 found in the alveolar region one day post-exposure [78]. In a 2010 study, Porter et al. showed that mice exposed to $10,20,40$ or $80 \mu \mathrm{g}$ of pristine MWCNT-7 exhibited short-term polymorphonuclear leukocyte infiltration and lactate dehydrogenase release, and early signs of pulmonary fibrosis at 7 days post-exposure [40]. A recent inhalation study in mice suggests that MWCNT-7 are biopersistent, can remain in the lung, and may travel throughout the periphery approximately one year post-exposure [79]. 
A review of MWCNT in vitro studies indicated that exposures up to $200 \mu \mathrm{g} / \mathrm{mL}$ of MWCNT are commonly used which are irrelavent to doses achieved within the mouse and human lung [10]. However, some in vitro studies utilize lower exposures ranging from $0.1 \mu \mathrm{g} / \mathrm{mL}$ to $6 \mu \mathrm{g} / \mathrm{mL}$ that further support the in vivo results, suggesting that pristine MWCNT, including MWCNT-7, are capable of inducing molecular signals potentially responsible for the toxic effects observed in vivo $[19,91,92,108$ 111]. In this study, the dosage of $1.2 \mu \mathrm{g} / \mathrm{ml}$ of MWCNT-7 or ND-MWCNT was selected to be reflective of previously conducted in vivo studies, roughly correlating with a $60 \mu \mathrm{g}$ dose of MWCNT in mice based upon the alveolar surface area of the mouse lung and surface area of a cell culture dish (Porter et al. 2010). These in vivo doses were roughly reflective of lung burdens due to concentrations of airborne MWCNT measured in actual workplaces and account for MWCNT mass median aerodynamic diameter, minute ventilation, and human alveolar epithelium surface area [46, 91].

The addition of defects or heteroatoms, such as nitrogen, to MWCNT can change their crystallinity and reactivity for industrial use, and may also impact their bioactivity. ND-MWCNT have been synthesized using a variety of starting materials, catalysts, and methods, resulting in CNT with varying percentages of nitrogen and levels of disruption to the graphene lattice. ND-MWCNT are suspected to grow by a base-growth model limited by diffusion of carbon atoms towards the catalyst molecules, coverage of the catalyst sites by amorphous carbon, or constraints brought on by pyridinic $\mathrm{N}$ incorporation $[75,112]$. $\mathrm{N}$-incorporation can be pyridinic $\left(\mathrm{sp}^{2}\right.$ coordinated, $\mathrm{N}$ atom part of hexagon ring connecting two $\mathrm{C}$ atoms) or pyrrolic ( $\mathrm{sp}^{3}$ coordinated, $\mathrm{N}$ atom part of pentagon ring connecting two $\mathrm{C}$ atoms) depending on the percentage of nitrogen in the CNT and the doping conditions. Therefore, since ND-MWCNT fabrication is not standardized, physicochemical characterization is of great importance for understanding their biological effects and potential toxicities.

Traditional pristine MWCNT have limited electrical conductance compared to doped variants; however, the addition of nitrogen to the carbon lattice confers MWCNT n-type semiconductor 
properties [113]. ND-MWCNT typically lack a hollow core common to pristine MWCNT and have bamboo-like sections walled off by layers of graphene. Nitrogen creates defects in the MWCNT by forcing rearrangement of the carbons around the heteroatoms, thereby introducing disorder into the graphene layers and curvature of the CNT [113]. Since nitrogen has one additional electron as compared to carbon, it is believed that this extra electron can carry current and also potentially interact with ROS, which are highly unstable radicals containing at least one oxygen atom. Tsuruoka et al. suggested that unpaired electrons on the surface of MWCNT may participate in reactions with ROS [114]. They assessed a variety of MWCNT with different modifications for ROS quenching through electron spin resonance studies, and showed that doping and surface chemistry altered the ability of CNT to quench ROS. The ability to produce ROS is a key characteristic of MWCNT that can be controlled by surface modification and composition and directly alters MWCNT-induced potential toxicity in biological systems. Addition of functional groups to the surface of MWCNT, such as nitrogen groups, carboxyl groups, polyethelene glycol, or others, can also alter the uptake and biocompatibility of MWCNT as recently shown by Li et al. [68].

ND-MWCNT are used in a variety of applications including gas sensors, matrix fillers in composite materials, field emission devices, and improved lithium storage in lithium batteries; however, information on ND-MWCNT bioactivity is limited [113]. Carrero-Sanchez et al. showed that ND-MWCNT (2-4\% nitrogen) are associated with lesser toxicities and pathological conditions compared to pristine CNT in an in vivo rat model after nasal, oral, intratracheal, and intraperitoneal exposures up to $5 \mathrm{mg} / \mathrm{kg}$. Only the highest dose $(5 \mathrm{mg} / \mathrm{kg}$ ) led to hyperplasia, inflammatory cell infiltration, fibroblastic proliferation, and some granulomas in ND-MWCNT treated rats, but was lethal in pristine CNT-exposed animals [70]. Limited in vitro results suggest their biocompatibility with blood cells with minimal toxicity at higher doses $(20 \mu \mathrm{g} / \mathrm{ml})$, which could be useful for potential intravenous drug delivery [115]. Elias et al. also demonstrated that ND-MWCNT are the only type of CNT tested that do not reduce cell viability 
in amoeba (even at higher doses), thus indicating that these ND-MWCNT are less toxic when compared to other types [116]. However, others have shown that tube length may play a key role in toxicity, with longer ND-MWCNT being more toxic than other functionalized CNT [117].

The present study assessed the bioactivity of ND-MWCNT and MWCNT-7 at low exposures in hTERT immortalized human small airway epithelial cells (SAEC) derived from the lower bronchioles. Through assessment of physical characteristics, uptake into SAEC, cell viability, ROS production, and cell cycle analysis, we provide an in vitro benchmark for ND-MWCNT toxicity as compared to MWCNT-7 in a model of lung epithelium.

\section{Materials and Methods}

\subsection{ND-MWCNT and MWCNT-7}

The ND-MWCNT used in this study were a gift from Mauricio Terrones (Pennsylvania State University, University Park, PA), and Morinobu Endo, and Shuji Tsuruoka (Shinshu University, Nagano, Japan). NDMWCNT were characterized at the Morgantown National Institute for Occupational Safety and Health and West Virginia University Shared Research Facilities. The MWCNT-7 used in this study were originally obtained through the Mitsui \& Co., Ltd. (MWCNT-7, lot \#05072001K28) and previously characterized (Porter et al. 2010).

\subsection{ND-MWCNT and MWCNT-7 preparation}

For cell culture studies, ND-MWCNT were prepared in dispersion media (DM) consisting of PBS (pH 7.4, $\mathrm{Ca} / \mathrm{Mg}$-free) supplemented with $0.6 \mathrm{mg} / \mathrm{ml}$ mouse serum albumin and $0.01 \mathrm{mg} / \mathrm{ml}$ 1,2-dipalmitoyl-snglycero-3-phosphocholine (DPPC) as previously described [118]. DPPC was prepared fresh as a $1 \mathrm{mg} / \mathrm{ml}$ stock solution in $100 \%$ ethanol. Transmission electron microscopy (TEM) micrographs of MWCNT dispersed in DM demonstrated that DM promotes significant dispersion of MWCNT [118]. ND-MWCNT and MWCNT-7 were prepared in DM by indirect sonication at $4^{\circ} \mathrm{C}$ for 5 min (Hielscher ultrasonic processor, UIS259L; Ringwood, NJ) at amplitude 100\% and cycle 1. Following indirect sonication, the 
suspension was directly sonicated for 5 min at $5 \mathrm{~W}$ output and $30 \%$ duty cycle in 1 min increments (Branson Sonifier 450; Danbury, CT). The stock solution $(0.5 \mathrm{mg} / \mathrm{ml}$ ) of ND-MWCNT or MWCNT-7 was kept at $4^{\circ} \mathrm{C}$ and used within 2-3 weeks. The ND-MWCNT and MWCNT-7 stock solutions were directly sonicated for $1 \mathrm{~min}$ at $5 \mathrm{~W}$ output and 30\% duty cycle prior to cell culture experiments. Cells were treated with DM, ND-MWCNT, or MWCNT-7 at $1.2 \mu \mathrm{g} / \mathrm{ml}$, a concentration which was extrapolated from previous murine in vivo exposure experiments based upon alveolar surface area [40].

\subsection{Cell culture}

SAEC were a gift from Tom K. Hei (Columbia University, New York, NY) [119]. SAEC were cultured in serum-free SABM medium supplemented with a SAGM SingleQuot kit of growth factors, cytokines, and supplements (Lonza Walkersville, Inc.; Walkersville, MD). SAEC were maintained in an incubator at $37^{\circ} \mathrm{C}$ with $5 \% \mathrm{CO}_{2}$. For cell culture experiments, SAEC were serum starved $24 \mathrm{~h}$ prior to ND-MWCNT or MWCNT-7 exposure in serum-free SABM medium.

\subsection{Transmission Electron Microscopy (TEM)}

Samples of ND-MWCNT were diluted in double distilled $\mathrm{H}_{2} \mathrm{O}\left(\mathrm{dd}_{2} \mathrm{O}\right)$ followed by vortexing, and a drop of solution was placed on a formvar-coated copper grid and allowed to air dry. Images were photographed under a JEOL 1220 Transmission Electron Microscope (Peabody, MA). Additionally, SAEC interaction with and engulfment of ND-MWCNT and MWCNT-7 were then analyzed by TEM. SAEC were grown to confluence and exposed to $1.2 \mu \mathrm{g} / \mathrm{ml} \mathrm{DM}$, ND-MWCNT, or MWCNT-7 for 6 or $24 \mathrm{~h}$. Cells were trypsinized with ReagentPack subculturing reagents (Lonza Walkersville, Inc., Walkersville, MD) per manufacturer guidelines and harvested by centrifugation at $400 \times \mathrm{g}$ for $5 \mathrm{~min}$. Cells were fixed in Karnovksy's fixative (2.5\% glutaraldehyde and 3\% paraformaldehyde in $0.1 \mathrm{M}$ sodium cacodylate, $\mathrm{pH}$ 7.4), washed three times in $0.1 \mathrm{M}$ sodium cacodylate, and postfixed in $1 \%$ osmium tetraoxide, followed by washing with $0.1 \mathrm{M}$ sodium cacodylate and distilled water. The cells were dehydrated by sequential 
washings in 25, 50, and 100\% ethanol and embedded in LX-112 (Ladd; Williston, VT). Ultrathin sections were stained with uranyl acetate and lead citrate and examined with a JEOL 1220 transmission electron microscope.

\subsection{Field Emission Scanning Electron Microscopy (FESEM)}

The particles were dispersed in $\mathrm{ddH}_{2} \mathrm{O}$ and filtered with a $0.2 \mu \mathrm{m}$ nucleopore filter. The filter was attached with double-stick carbon tape on an aluminum mount and sputter coated with gold/palladium. Images were collected on a Hitachi (Tokyo, Japan) S-4800 field emission scanning electron microscope.

\subsection{X-ray Photoelectron Spectroscopy (XPS)}

XPS analysis was carried out with a Physical Electronics VersaProbe 5000 XPS (Chanhassen, MN). NDMWCNT powder was pressed into a small pellet and evacuated in an entry chamber for approximately 2 $\mathrm{h}$ before being transferred to an ultra-high vacuum chamber for analysis. The sample was stimulated by a focused Aluminum K- $\alpha$ X-ray source at $1486 \mathrm{eV}$ energy and $25 \mathrm{~W}$ power with an X-ray spot size of 100 ?m. A survey scan was carried out using an analyzer pass energy of $117.40 \mathrm{eV}$, and high-resolution scans for carbon, oxygen, and nitrogen elements were carried out at an analyzer pass energy of $23.50 \mathrm{eV}$. The spectra were referenced to the C1s peak at a binding energy of $284.8 \mathrm{eV}$.

\subsection{Raman Spectroscopy}

Raman spectra of the MWCNT were collected using a Renshaw Invia Raman Spectrometer with a CCD detector (Hoffman Estates, IL). ND-MWCNT or MWCNT-7 powders were directly placed on a microscope glass slide, which was then mounted under the $50 \times$ objective lens of the Raman microscope. Samples were excited with a $532 \mathrm{~nm}$ green laser at around $0.23 \mathrm{~mW}$ laser power for data collection.

\subsection{X-Ray Diffraction (XRD)}

XRD patterns of the MWCNT were collected with a PANanalytical X'Pert Pro powder X-ray diffractometer. ND-MWCNT or MWCNT-7 powders were filled into stainless steel powder sample holders and radiated with a Cu K- $\alpha$ X-ray source at $1.8 \mathrm{~kW}$ power in a theta/theta scan mode. The XRD 
spectra of the samples were collected over a 2-theta range of $15^{\circ}$ to $90^{\circ}$ at a step size of $0.05^{\circ}$ with a solid state X-ray detector.

\subsection{Zeta Potentials}

The electrophoretic mobility (motion of particles relative to a fluid under the influence of an electric field) of particles was determined using light scattering in an applied electric field and converted to values of zeta potential (the potential difference between a dispersion medium and stationary layer of fluid attached to a particle in the medium) using the Henry equation. For each material, an independent sample was drawn for determination of electrophoretic mobility. The instrument operability was previously verified using U.S. National Institute of Standards and Technology Standard Reference Material 1980: Positive Electrophoretic Mobility, having a certified value for mobility of $2.53 \pm 0.12$ $\mu \mathrm{m} \cdot \mathrm{cm} /(\mathrm{V} \cdot \mathrm{s})$, corresponding zeta potential of $\sim 32.5 \mathrm{mV}$ at $25^{\circ} \mathrm{C}$, and prepared using the protocol described in the SRM certificate. The $\mathrm{pH}$ of the experimental samples was determined after measurement of electrophoretic mobility using a calibrated electrode attached to a volt meter. The $\mathrm{pH}$ probe tip was rinsed thoroughly with $18 \mathrm{M} \Omega-\mathrm{cm}$ ddH ${ }_{2} \mathrm{O}$ and blotted dry before measurements. The PBS had a pH of 7.2, and SAEC serum-free media had a pH of 7.6. The parameters for the dispersants were based on PBS (refractive index $=1.334$, viscosity $=0.9110 \mathrm{CP}$, dielectric constant $=79.0$, and Smoluchowski approximation, $\mathrm{f}(к а)$ value $=1.5)$. Zeta potential is used as an indicator of the stability characteristics of a dispersion. Riddick developed categories to describe the stability of dispersions based on zeta potential: threshold of agglomeration (-10 to $-15 \mathrm{mV})$, threshold of delicate dispersion (16 to $-30 \mathrm{mV})$, moderate stability (-31 to $-41 \mathrm{mV}$ ), fairly good stability (-41 to $-60 \mathrm{mV})$, and very good stability (-61 to $-80 \mathrm{mV}$ ) (Riddick 1968). All measurements were performed at $25^{\circ} \mathrm{C}$ using a Malvern Zetasizer Nano ZS90 (Worcestershire, UK) equipped with a $633 \mathrm{~nm}$ laser at a $90^{\circ}$ scattering angle. Samples were equilibrated inside the instrument for two min, and five measurements (10 sec 
delay between measurements), each consisting of five runs (two sec delay between runs), were recorded.

\subsection{ROS production}

ROS measurements by confocal microscopy were performed according to methods previously described (Qian et al. 2010). SAEC were cultured on glass cover slips, serum starved $24 \mathrm{~h}$, and exposed to 1.2

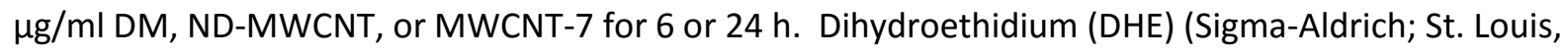
$\mathrm{MO}$ ) was added at a final concentration of $5 \mu \mathrm{M}$ for the last $30 \mathrm{~min}$ of exposure in the dark. After incubation, cells were washed twice with PBS ( $\mathrm{pH} 7.4$ ), fixed with $4 \%$ paraformaldehyde, washed three times over 15 min with PBS, and mounted on slides with ProLong Gold anti-fade (Invitrogen; Carlsbad, CA). A Zeiss LSM 510 microscope was used to obtain images with a 20x objective. DHE staining was determined by fluorescence at $546 \mathrm{~nm}$. Five independent experiments were carried out with representative images taken from each slide. Images from a single experiment overall reflective of these experiments are found in Figure 7.

\subsection{Cytotoxicity assay}

A dose-response assessment of cytotoxicity was carried out by seeding SAEC ( $1 \times 10^{4}$ cells) in $100 \mu l$ complete media in 96-well plates, followed by $24 \mathrm{~h}$ serum starvation prior to exposure to DM, NDMWCNT, or MWCNT-7 for $24 \mathrm{~h}$ at $0,0.12,1.2,12$, or $120 \mu \mathrm{g} / \mathrm{ml}$. Twenty microliters of CellTiter $96^{\circledR}$ Aqueous One Solution (Promega; Madison, WI) was added to each well during the last 4 hours of exposure. Absorbance was read at $490 \mathrm{~nm}$ using a BioTek Synergy H1 plate reader (Winooski, VT), and statistical analyses were carried out using the Data Analysis pack in Microsoft Excel (Redmond, WA). Three experiments were performed in triplicate; treatment groups were averaged by triplicate and compared using two sample t-tests assuming unequal variances. Error bars are reflective of standard error. 


\subsection{Cell Cycle Assessment}

SAEC were grown to subconfluency, serum starved for $24 \mathrm{~h}$, and treated with $1.2 \mu \mathrm{g} / \mathrm{ml} \mathrm{DM}, \mathrm{MWCNT}-7$, or ND-MWCNT. Cells were collected by trypsinization, followed by centrifugation at $1100 \times \mathrm{g}$ for $7 \mathrm{~min}$. Cell pellets were washed in PBS and subsequently resuspended in $100 \mu \mathrm{L}$ PBS. Cells were fixed in $70 \%$ ethanol and stored at $4^{\circ} \mathrm{C}$ until assessed by flow cytometry. Fixed SAEC were centrifuged and washed twice with PBS, treated with $50 \mu \mathrm{L}$ of $100 \mu \mathrm{g} / \mathrm{ml}$ RNase (Sigma-Aldrich, St. Louis, MO), and stained with a final concentration of $10 \mu \mathrm{g} / \mathrm{ml}$ propidium iodide (Sigma-Aldrich, St. Louis, MO). Samples were run on an LSRII cell analyzer (BD Biosciences, San Jose, CA). Ten thousand events were collected per sample, and analysis was carried out using FlowJo software (Ashland, OR). Gating was set to exclude debris and only include cells by forward and side scatter. Gating for singlet discrimination was through pulse width versus area for phycoerythrin, forward scatter, and side scatter. The Watson model was used to determine the percentage of cells in each phase. Cells in each phase were analyzed from two independent trials in biological triplicate $(n=6)$ from which 10,000 events were collected from each individual sample. Statistical analysis was done using the Data Analysis pack in Microsoft Excel (Redmond, WA). Treatment groups were compared using two sample t-tests assuming unequal variances and error is presented as standard error in Table 2.

\subsection{Western Blotting}

Whole cell protein extraction was carried out using RIPA buffer (150 mM NaCl, 10 mM Tris pH 7.4, 2 mM EDTA, 1\% IGEPAL, $1 \%$ sodium deoxycholate, $0.1 \%$ sodium dodecyl sulfate) supplemented with a 10 $\mu \mathrm{g} / \mathrm{ml}$ protease inhibitor cocktail and $10 \mu \mathrm{g} / \mathrm{ml}$ phosphatase inhibitor (Thermo Fisher Scientific; Waltham, MA). Protein concentration was measured using a BCA protein assay kit (Thermo Fisher Scientific, Waltham, MA). Twenty micrograms of protein per sample was resolved using sodium dodecyl sulfate-polyacrylamide gel electrophoresis (SDS-PAGE) and transferred to polyvinylidene fluoride membranes (PVDF, Pall Corporation; Pensacola, FL). Membranes were blocked for $1 \mathrm{~h}$ in $5 \%$ bovine 
serum albumin (Thermo Fisher Scientific, Waltham, MA) in Tris Buffered Saline with Tween-20 (TBST) (62.5mM Tris pH 7.4, 150mM NaCl, 0.05\% Tween 20) (Thermo Fisher Scientific, Waltham, MA) and incubated with CDK4, phospho-tyrosine, or phospho-threonine (Cell Signaling Technology, Boston, MA) primary antibodies overnight at $4^{\circ} \mathrm{C}$. Membranes were washed three times with TBST for ten min each and incubated with species-specific secondary antibodies for $1 \mathrm{~h}$ at room temperature. Analysis was carried out using ECL Western Blotting Substrate (Thermo Fisher Scientific, Waltham, MA). Representative western blots are shown in Figure 8 and 9 from a minimum of three independent experiments per antibody. Densitometry was carried out using ImageJ software (NIH, Bethesda, MD) on three representative western blots normalized to respective $\beta$-actin blots per antibody. Error bars in the bar graphs show standard error.

\section{Results}

\subsection{Characteristics of ND-MWCNT and MWCNT-7 in suspension}

Low magnification TEM (Figure 2.1 A, B) and FESEM (Figure 2.1 C, D) indicated that the ND-MWCNT were $5.28 \pm 2.07 \mu \mathrm{m}$ long and $79.7 \pm 19.4 \mathrm{~nm}$ wide, after assessment for size in Adobe Photoshop CS5 (San Jose, CA). The ruler tool was used to measure single nanotubes from the TEM and SEM images. As previously determined, the MWCNT-7 were determined to be 3.86 (GSD 1.94) $\mu \mathrm{m}$ long and $49 \pm 13.4 \mathrm{~nm}$ wide [40] (Table 1). Zeta potentials were measured for ND-MWCNT and MWCNT-7 in DM diluted in PBS and serum free SAEC media. In PBS ( $\mathrm{pH} 7.2$ ), values were determined to be $-13.9 \pm 0.485 \mathrm{mV}$ and $-15.8 \pm$ $0.450 \mathrm{mV}$, respectively. In serum free media $(\mathrm{pH} 7.6)$, values were determined to be $-12.9 \pm 0.835 \mathrm{mV}$ and $-12.2 \pm 0.283 \mathrm{mV}$, respectively (Table 2.1), and there was no appreciable difference in zeta potentials between particles in PBS and those in serum free SAEC media. 


\subsection{Physical characteristics of ND-MWCNT and MWCNT-7}

Several analytic methods were used to assess the physical characteristics of the ND-MWCNT and MWCNT-7, including XPS, Raman spectroscopy, and XRD. The MWCNT-7 were previously characterized by Porter et al. [40] as pristine MWCNT with 20 to 50 walls per nanotube and with minimal trace metal contamination, including sodium (0.41\%) and iron (0.32\%). XPS determined the elemental composition and surface chemistry of the ND-MWCNT. The XPS survey scan revealed the existence of carbon, oxygen, and nitrogen elements within ND-MWCNT. The corresponding atomic concentration was determined from high resolution spectra of carbon, oxygen, and nitrogen elements, showing that the ND-MWCNT were composed of approximately 93.3\% carbon, 3.8\% oxygen and $2.9 \%$ nitrogen (Figure $\mathbf{2 . 2}$ A). The spectra of these elements were analyzed, and different chemical states were identified. The carbon atoms were found to be in three functional groups: $\mathrm{sp}^{2}$-hybridized graphitic carbon $(284.8 \mathrm{eV}), \mathrm{C}-$ $\mathrm{N}(285.9 \mathrm{eV})$, and $\mathrm{C}=\mathrm{O}(287.3 \mathrm{eV})$ (Figure 2.2 B). The nitrogen atoms were found to be in two chemical states: amino $-\mathrm{NH}_{2}(399.0 \mathrm{eV})$ and pyrrolic C-N (400.3 eV) types (Seran et al. 2012; Zhang et al. 2013) (Figure 2.2 D). The oxygen atoms were found to be in two chemical states: $\mathrm{C}=\mathrm{O}(531.8 \mathrm{eV})$ and $-\mathrm{OH}$ (533.6 eV) (Figure 2.2 C). These XPS results further showed the inclusion of nitrogen atoms into the graphitic carbon lattice of ND-MWCNT (pyrrolic C-N bonding) and the existence of lattice structural disorder.

To further characterize these materials, Raman spectroscopy was used to assess the carbon lattice structure condition of ND-MWCNT and MWCNT-7 samples. Raman peaks at around $1358 \mathrm{~cm}^{-1}, 1590 \mathrm{~cm}$ ${ }^{1}$, and $2700 \mathrm{~cm}^{-1}$ were identified and assigned to the D-band, G-band, and $2 \mathrm{D}\left(\mathrm{G}^{\prime}\right)$-band of carbon structure (Ferrari and Basko 2013). The intensity ratio of the defect-derived D-band to the G-band (ID/IG), an indicator of the structural integrity of the graphitic carbon lattice, was calculated based on Raman peak fitting results. A D-band to G-band intensity ratio of 0.132 was obtained for MWCNT-7, 
while the ND-MWCNT had a ratio of 1.440, indicating significant structural disorder induced in the graphitic carbon lattice of the ND-MWCNT due to nitrogen doping (Figure 2.3).

XRD was performed to study the crystallinity condition of the MWCNT. The strong diffraction peaks at $26.3^{\circ}$ and $42.5^{\circ}$ were assigned to the (002) and (100) diffractions of the graphitic lattice of the MWCNT (Zhao et al. 2014). The full width at half maximum (FWHM) values of the (002) peak in MWCNT-7 and ND-MWCNT spectra were $0.91^{\circ}$ and $1.21^{\circ}$, respectively. Compared to MWCNT-7, the ND-MWCNT had a broader (002) peak with lower intensity, indicating a decrease of graphene crystallinity due to nitrogen doping induced structural damage (Figure 2.4).

\subsection{ND-MWCNT and MWCNT-7 exposure alters SAEC cytotoxicity}

A CellTiter $96^{\circledR}$ Aqueous One assay was carried out to determine the effects of ND-MWCNT and MWCNT-7 on SAEC proliferation and cytotoxicity. SAEC were treated with DM or $0.12,1.2,12$, and 120 $\mu \mathrm{g} / \mathrm{ml}$ of ND-MWCNT or MWCNT-7 for $24 \mathrm{~h}$. SAEC treated with $0.12,1.2$, and $12 \mu \mathrm{g} / \mathrm{ml}$ ND-MWCNT exhibited a trend towards increased proliferation, which decreased at $120 \mu \mathrm{g} / \mathrm{ml}$ when compared to DM. Treatment of SAEC with MWCNT-7 at $0.12 \mu \mathrm{g} / \mathrm{ml}$ significantly increased proliferation compared to DM and $0.12 \mu \mathrm{g} / \mathrm{mL}$ ND-MWCNT, while treatment with $1.2 \mu \mathrm{g} / \mathrm{ml}$ and $12 \mu \mathrm{g} / \mathrm{ml}$ exhibited a lessened proliferative effect compared to DM. At $120 \mu \mathrm{g} / \mathrm{ml}$, MWCNT-7 significantly induced cytotoxicity below that of DM (Figure 2.5). In summary, MWCNT-7 significantly enhanced epithelial cell proliferation at a lower dose than ND-MWCNT. Additionally, a high dose of $120 \mu \mathrm{g} / \mathrm{mL}$ of MWCNT-7 was cytotoxic to the SAEC, while ND-MWCNT was not. Therefore, MWCNT-7 appeared more potent in inducing proliferation and cytotoxicity than ND-MWCNT. A dose of $1.2 \mu \mathrm{g} / \mathrm{ml}$ was selected for further studies as it was previously established as an occupationally relevant dose capable of inducing cellular effects in SAEC upon treatment with the particles studied here $[19,91]$. Also, no significant difference was noted between proliferation of SAEC treated with $1.2 \mu \mathrm{g} / \mathrm{ml}$ of ND-MWCNT or MWCNT-7. 


\subsection{ND-MWCNT and MWCNT-7 uptake by SAEC}

SAEC were treated with DM (Figure 6A), ND-MWCNT (Figure 2.6 B, C, D), or MWCNT-7 (Figure 2. 6 E, F, G) and imaged for particle interaction and uptake through TEM. ND-MWCNT and MWCNT-7 varieties were found at the cell surface at $6 \mathrm{~h}$ (Figure 2.6 B, C or E, F, respectively), followed by internalization at 24 h (Figure 2.6 D or G, respectively).

\subsection{ND-MWCNT and MWCNT-7 exposure induces ROS production in SAEC}

Some nanomaterials, including MWCNT-7, are capable of inducing the production of ROS upon interaction with various cell types [91, 120-123]. In this experiment, ND-MWCNT and MWCNT-7 were examined to determine their ability to induce ROS production in SAEC. SAEC exposed to DM (Figure 2.7 A), ND-MWCNT (Figure 2.7 B, C), or MWCNT-7 (Figure 2.7 D, E) were stained with DHE to assess ROS production at 6 and $24 \mathrm{~h}$. ROS production peaked at $6 \mathrm{~h}$ and remained higher than control at $24 \mathrm{~h}$ in ND-MWCNT treated cells (Figure 2.7 B, C). MWCNT-7 appeared to induce ROS production at $6 \mathrm{~h}$ that resolved to baseline levels at $24 \mathrm{~h}$ (Figure 2.7 D, E). The more rapid response to MWCNT-7 is consistent with greater potency compared to ND-MWCNT.

\subsection{ND-MWCNT and MWCNT-7 exposure affects SAEC cell cycle and CDK4 expression}

The cell cycle, which regulates cell duplication and division, can be altered by MWCNT [82, 108, 124]. Here, the effects of ND-MWCNT and MWCNT-7 on the cell cycle were compared. SAEC were treated with DM, ND-MWCNT, and MWCNT-7 for 6 or $24 \mathrm{~h}$ prior to fixation and cell cycle analysis. Propidium iodide staining was used to examine potential ND-MWCNT and MWCNT-7-related changes to the cell cycle. Results are presented as percentage of cells in each phase in Table 2.2. Results indicate that MWCNT-7 induce a significant change in the percentage of cells in G2 compared to DM at $6 \mathrm{~h}$, suggestive of cell cycle dysfunction. At $24 \mathrm{~h}$, a significant difference in percentage of cells in $\mathrm{G} 1$ and $\mathrm{G} 2$ was noted in SAEC exposed to ND-MWCNT compared to MWCNT-7. A significant increase was noted between 6 and $24 \mathrm{~h}$ in G2 in ND-MWCNT exposed cells. Significant changes were noted between 6 and 
$24 \mathrm{~h}$ in G1 and S phase in MWCNT-7 exposed cells (Table 2.2). To gain a better understanding of a molecular basis for the cell cycle dysfunction, whole cell lysates were subjected to SDS-PAGE and probed for CDK4, a serine/threonine cyclin dependent kinase important to the G1 transition prior to synthesis of new DNA in S phase (Baker and Reddy 2012). We observed an increase in CDK4 expression in SAEC exposed to ND-MWCNT over $6 \mathrm{~h}$, followed by a decrease at $24 \mathrm{~h}$, which is reflective of the propidium iodide cell cycle analysis results. CDK4 does not appear to play a role in the molecular mechanism employed by MWCNT-7 to induce cell cycle arrest, which was expected because CDK4 should not be active during G2 (Figure 2.8).

\subsection{ND-MWCNT and MWCNT-7 exposure induces phospho-tyrosine and phospho-threonine}

The majority of cell signaling processes involve protein phosphorylation, especially signal transduction pathways and those essential to cell cycle regulation, cell differentiation, and cell maintenance [125, 126]. To better understand how ND-MWCNT and MWCNT-7 may affect these processes, general cell signaling pathways were probed using pan-phospho-tyrosine and phospho-threonine antibodies. SAEC were exposed to DM, ND-MWCNT, or MWCNT-7 over 6 or 24 h, and whole cell lysates were collected using RIPA buffer with protease and phosphatase inhibitors. Lysates were subjected to SDS-PAGE, transferred to PVDF membranes, and exposed to phospho-antibodies. Phospho-tyrosine expression increased at $6 \mathrm{~h}$ for MWCNT-7 exposed SAEC, suggesting a time-dependent activation of signaling pathways (Figure 2.9 A). Changes in phospho-threonine expression following MWCNT exposure were less obvious (Figure 2.9 B).

\section{Discussion}

Although MWCNT have been in production for over twenty years, functionalization offers an avenue to increase their dispersibility and industrial applications across a variety of fields. Surface functionalizations and substitution reactions (with nitrogen or boron, as examples) to the carbon lattice 
may increase nanotube reactivity at sites of curvature and breaks in the lattice $[127,128]$. The increase in porosity greatly increases the surface area (and therefore, reactive surface) of ND-MWCNT, which could have interesting implications for biocompatibility [128]. Nitrogen functionalization (or "doping") of CNT has been shown to significantly increase the brittleness, chemical reactivity, and n-type semiconductor activity of this material [129]. Varying amounts of nitrogen may be incorporated into the carbon lattice, disrupting $\mathrm{sp}^{2}$ bonding and creating potential active sites for further functionalization (e.g., drug molecules) or participation in oxygen reduction reactions $[129,130]$. Nitrogen-containing functional groups, such as amines, can also be attached to the outermost layer of graphene in CNT for use in biosensors and epoxies [131-133]. However, limited information is available on how functionalization of CNT with nitrogen alters their bioactivity.

Both pyridinic and pyrrolic nitrogen atoms are located at either the structural defect sites within the graphene network or at the sheet edges of the MWCNT walls. Specifically, our ND-MWCNT appear to have pyrrolic N-incorporation. The nitrogen atoms in MWCNT, especially at the outermost layer, become attraction sites for oxygen atoms and other functional groups and significantly modify MWCNT surface chemistry. The lengths of ND-MWCNT are often shorter than pristine MWCNT due to different growth kinetics, but was not observed in this study. Fiber length can play a role in toxicity and longer fibers have often been shown to be more toxic than their shorter counterparts $[89,134]$. Data from the present study suggesting that ND-MWCNT are less bioactive than pristine MWCNT are as follows: 1) In the CellTiter $96^{\circledR}$ Aqueous One assay, MWCNT-7 enhanced epithelial cell proliferation at a 10 -fold lower dose than ND-MWCNT; 2) MWCNT-7 caused significant cytotoxicity in the MTS assay at a dose of 120 $\mu \mathrm{g} / \mathrm{ml}$, while ND-MWCNT did not; and 3) MWCNT-7 induced an increase in phospho-tyrosine signaling at $6 \mathrm{~h}$ which may be linked to signaling cascades such as the MAPK and PI3K cascades [135]. Indications that ND-MWCNT may be less damaging than pristine MWCNT in vivo and in vitro have also been reported by other labs. Due to the inert character and agglomeration of pristine MWCNT, it is possible 
that the ND-MWCNT remain better dispersed in vivo and are therefore less likely to block the small airways in the lungs [70]. In vitro, the hydrophilic nature and surface charge of functionalized MWCNT, including ND-MWCNT, can alter their uptake and subsequent toxicity in pulmonary epithelial and macrophage cells. Previous studies have shown no significant activation of the NRLP3 inflammasome in cells exposed to nitrogen-functionalized MWCNT when compared to pristine MWCNT, making it a potentially better choice for use in industry to protect workers and the general public [68]. Boncel et al. studied the cytotoxicity of 3 wt. \% ND-MWCNT compared to oxidized MWCNT and pristine MWCNT and suggested that ND-MWCNT are of intermediate toxicity, but less toxic than the pristine variant [117].

In the present study, the bioactivities of ND-MWCNT and pristine MWCNT in short-term exposure of SAEC were compared. Our data suggests that ROS could potentially initiate the bioactivity noted in our study. It has been previously shown that MWCNT-7 produce no detectable hydroxyl radical alone or in the presence of $\mathrm{H}_{2} \mathrm{O}_{2}$ from the acellular Fenton reaction and instead scavenged ROS similar to ND-MWCNT which also scavenged ROS in the acellular Fenton reaction [40,91]. This implies that the SAEC were generating ROS in response to ND-MWCNT exposure. Within the past decade, ROS has been increasingly linked to a variety of cellular functions, including growth factor activation, cytoskeletal changes, and cell cycle progression, as well as contributing to inflammation, oxidative stress, and general cell signaling [135-138]. It has previously been shown that MWCNT are capable of inducing ROS in a variety of cell types $[41,91,139,140]$. Here, we showed that ND-MWCNT are capable of inducing ROS in SAEC in a time-dependent manner. ROS are an important part of cellular immunity, act as cell signaling agents, play a role in cytoskeletal modifications and cell cycle regulation, and can lead to the development of cancer [138]. Our results suggest that ND-MWCNT induced sustained production of ROS over a $24 \mathrm{~h}$ period, while MWCNT-7 induced transient production of ROS at $6 \mathrm{~h}$ that resolved by $24 \mathrm{~h}$. This difference may be due to the physicochemical properties of the particles and their ability to scavenge ROS, which has been shown to vary with nanotube composition, surface characteristics, and 
redox potential in acellular systems $[61,114]$. As suggested by Tsuruoka, surface electrons of MWCNT may be able to scavenge ROS in vivo through electron donation that could be limited by the ability of cells in the body to resupply electrons to MWCNT to scavenge additional ROS. When this theory is applied to our results, SAEC may be better able to resupply electrons on the surface of MWCNT-7 or MWCNT-7 may have more surface electrons available to donate to and quench ROS present in our cellular system over a $24 \mathrm{~h}$ period [100].

Although ROS can cause oxidative damage to DNA, lipids, and proteins, we believe that the sustained levels of ROS induced by ND-MWCNT treatment may be alternatively acting as a signaling molecule as proposed by Boonstra [137]. ROS can influence the cell cycle through the activation of growth factor receptors and phosphorylation and ubiquitination of cell cycle regulators [138]. The cell cycle is regulated through a series of "checkpoints" mediated by cyclins and serine/threonine cyclindependent kinases that tightly block or promote cycling. Several recent publications have discussed functionalized MWCNT-induced cell cycle alterations. Siegrist et al. studied carboxylated MWCNT and showed that exposure of BEAS-2B bronchial epithelial cells with $24 \mu \mathrm{g} / \mathrm{cm}^{2}$ MWCNT induced a significant increase in cells in S phase, suggesting a block at the G1/S checkpoint [82]. Zhang and Yan recently showed in several cell lines that carboxylated MWCNT caused cell cycle arrest at the transition between G1/S and a slight arrest in G2 in mouse mesenchymal stem cells and human neuroblastoma cells [124]. Our findings suggest that ND-MWCNT and MWCNT-7 may be inducing cell cycle aberrations between 6 and $24 \mathrm{~h}$ at $\mathrm{G} 2$ phase and at $6 \mathrm{~h}$ in $\mathrm{G} 2$ phase, respectively. Our findings also show that CDK4 levels distinctly vary between 6 and $24 \mathrm{~h}$ in SAEC exposed to ND-MWCNT or MWCNT-7, further suggesting that they may act through alternative mechanisms to alter the cell cycle. Total phospho-threonine levels are similar to CDK4 levels for each time point and exposure, suggesting that the cellular activation may be related. Phospho-tyrosine kinases are important for a variety of different cell signaling pathways including mitogen-activated protein kinase pathways, Jak/STAT pathways, and phosphotidylinoside 3- 
kinase [126]. These signaling pathways are related to cell cycle regulation, cell differentiation, cell migration, and apoptosis [141]. Since dysregulation of these pathways has been associated with inflammation, cancer, and diabetes, our results warrant further studies of these particles' specific molecular mechanisms. Our lab has previously shown significant increases in phospho-NF-kB p65, phospho-p38, and phospho-Stat3 in human microvascular endothelial cells indirectly exposed to MWCNT-7 in an alveolar-capillary co-culture system [19]. Others have also shown activation of p38 MAPK in BEAS-2B lung epithelial cells and normal mesothelial cells in response to MWCNT exposure, which may provide direction for future work $[142,143]$.

\section{Conclusions}

In this study, we present the first in-depth physicochemical characterization of these ND-MWCNT, suggesting that during synthesis, nitrogen atoms were incorporated into the MWCNT, introducing structural disorder and altering their crystallinity. Our major finding is that the differences in physicochemical properties, especially disruption of the carbon crystalline lattice by incorporation of nitrogen atoms, impacts the bioactivity of MWCNT. Overall, our findings suggest that the bioactivity of ND-MWCNT in SAEC is somewhat lower than that exhibited by pristine MWCNT due to differences in physicochemical properties. The time and dose-dependent nature of observed proliferation, cytotoxicity, ROS production, cell cycle alterations, and total phospho-tyrosine and phospho-threoninealtered proteins suggests that the physicochemical properties of MWCNT affect their subsequent biological effects.

\section{Acknowledgements}

A. L. Mihalchik acknowledges support from the National Science Foundation through the IGERT program under grant DGE-1144676. We would also like to acknowledge use of the WVU Shared Research Facilities. 


\section{Disclaimer}

The findings and conclusions in this report are those of the authors and do not necessarily represent the views of the National Institute for Occupational Safety and Health. The mention of Mitsui \& Co., Ltd. And MWCNT-7 does not constitute product endorsement. 

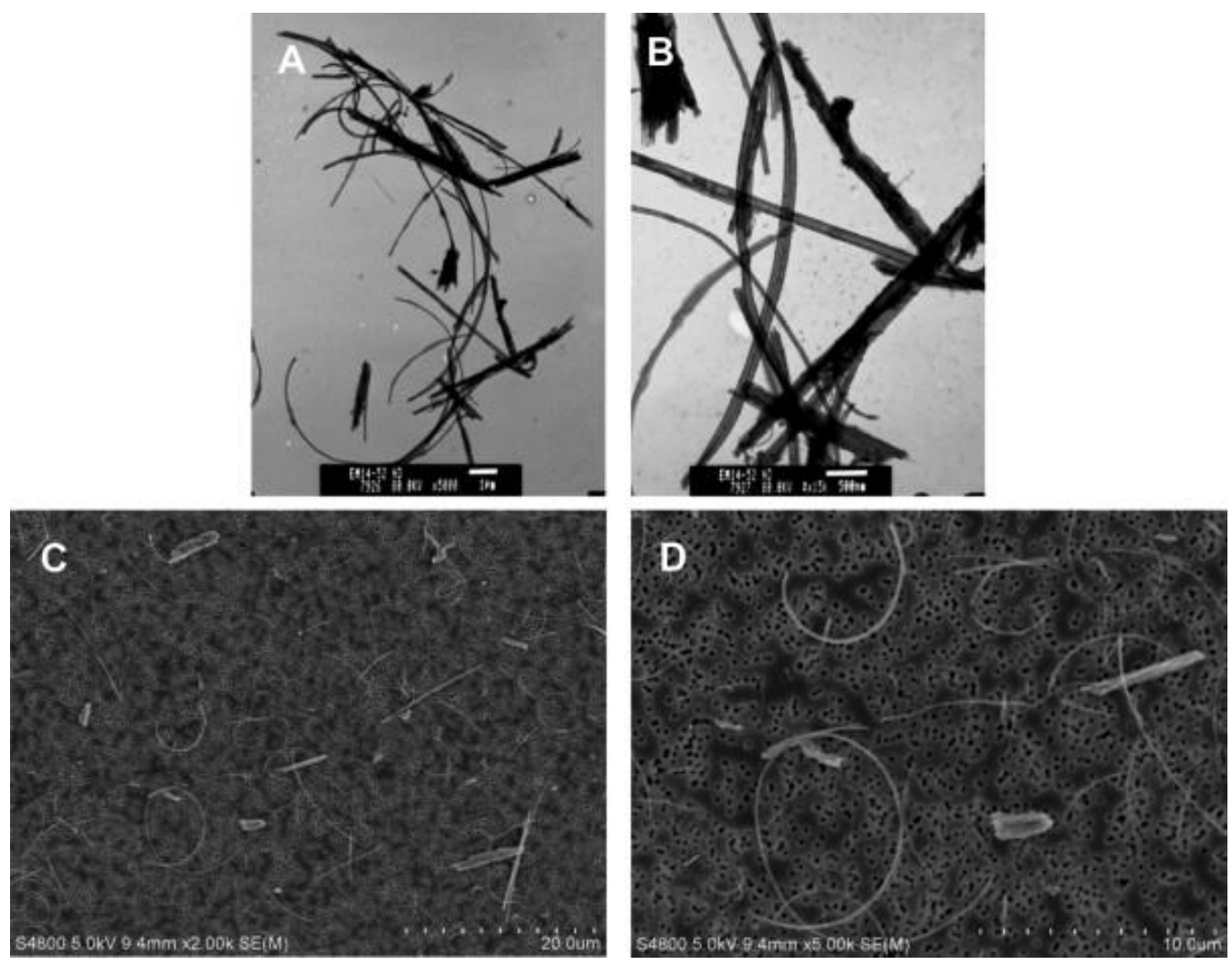

Figure 2.1: TEM of ND-MWCNT at 5000x (A) and 15000x (B) and field emission scanning electron micrographs of ND-MWCNT at 2000x (C) and 5000x (D). 

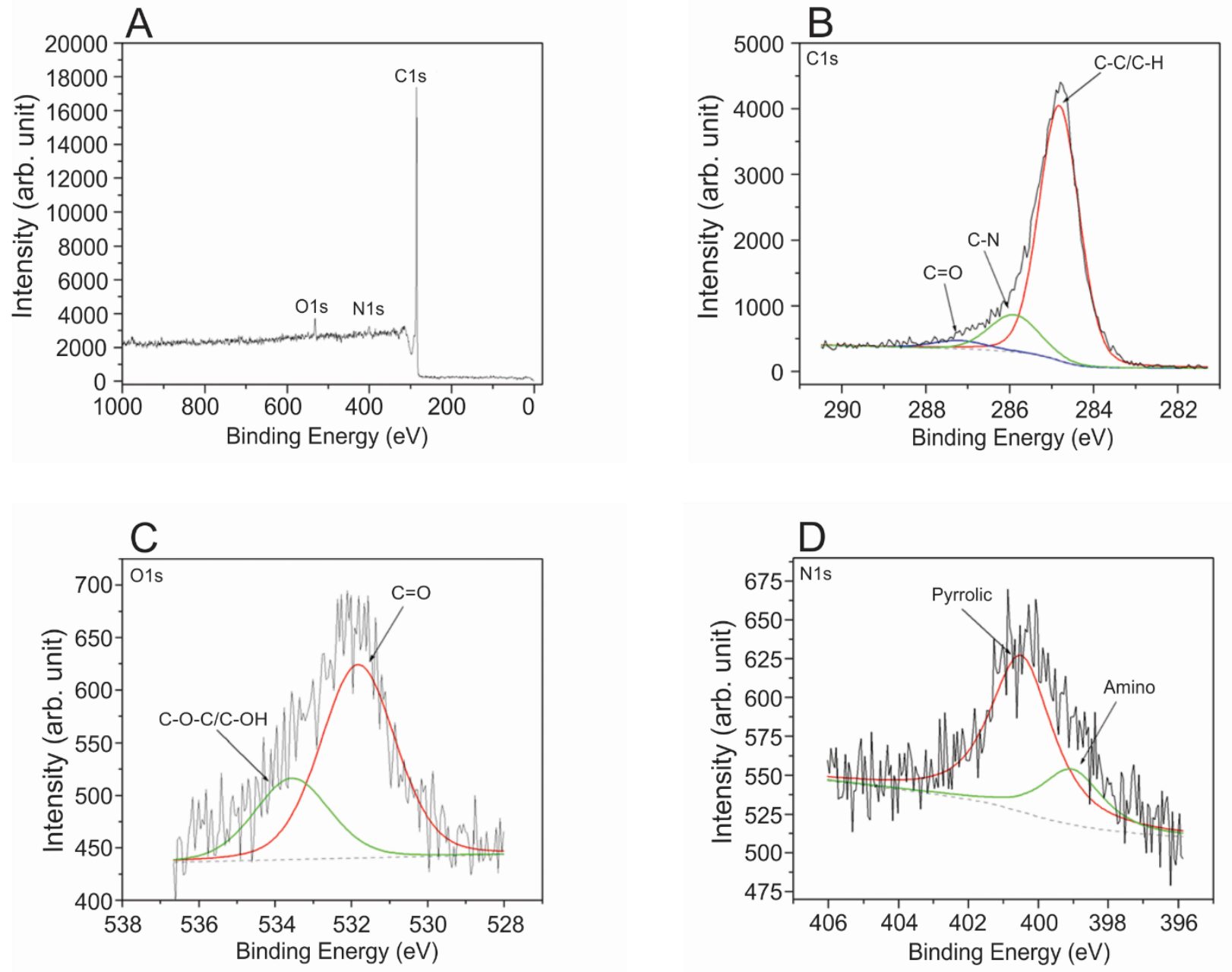

Figure 2.2: X-ray photoelectron spectroscopy survey scan spectrum of ND-MWCNT sample (A); high resolution X-ray photoelectron spectroscopy spectra for carbon (B), nitrogen (C), and oxygen elements (D). 


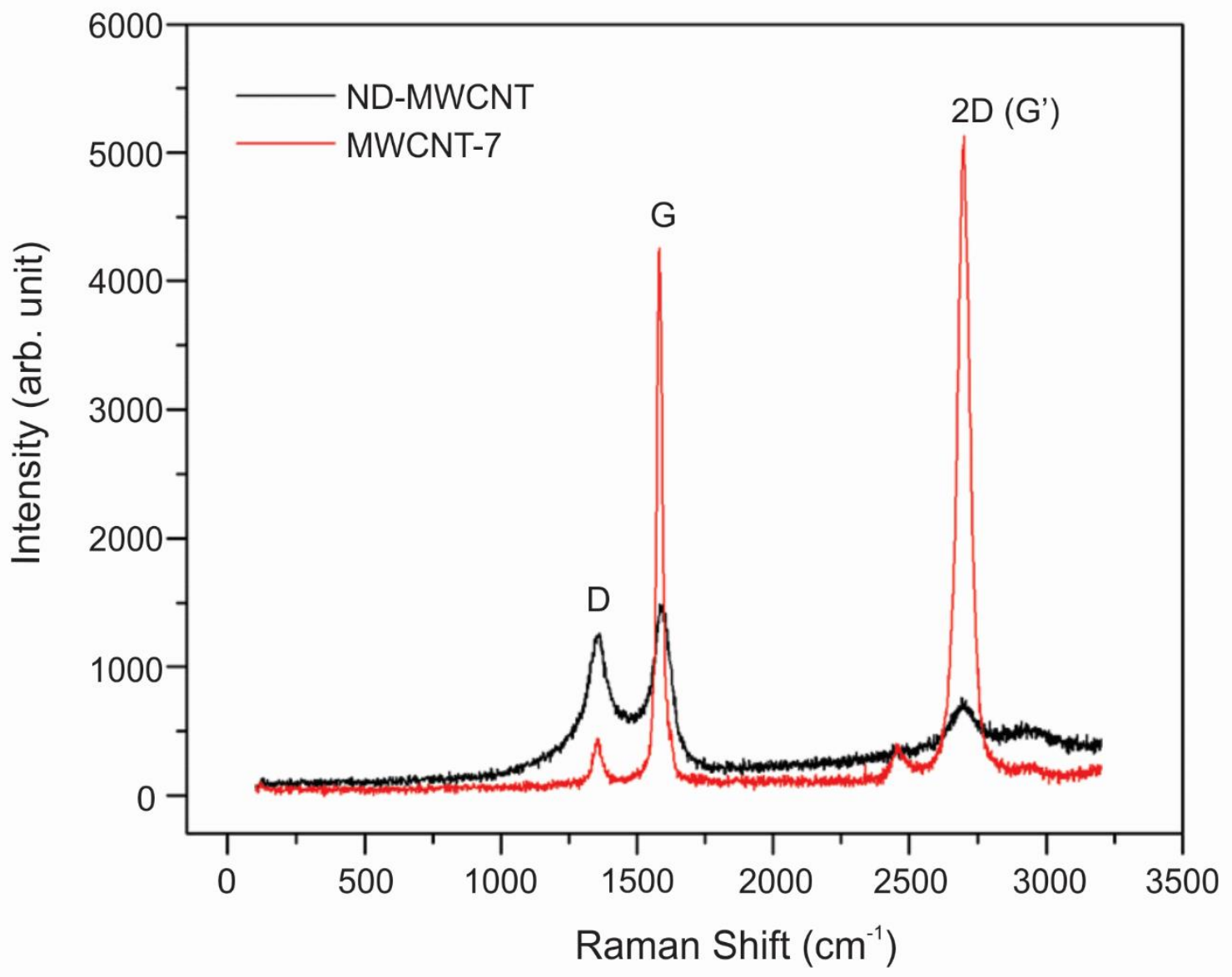

Figure 2.3: Raman spectra of ND-MWCNT and MWCNT-7 samples. 


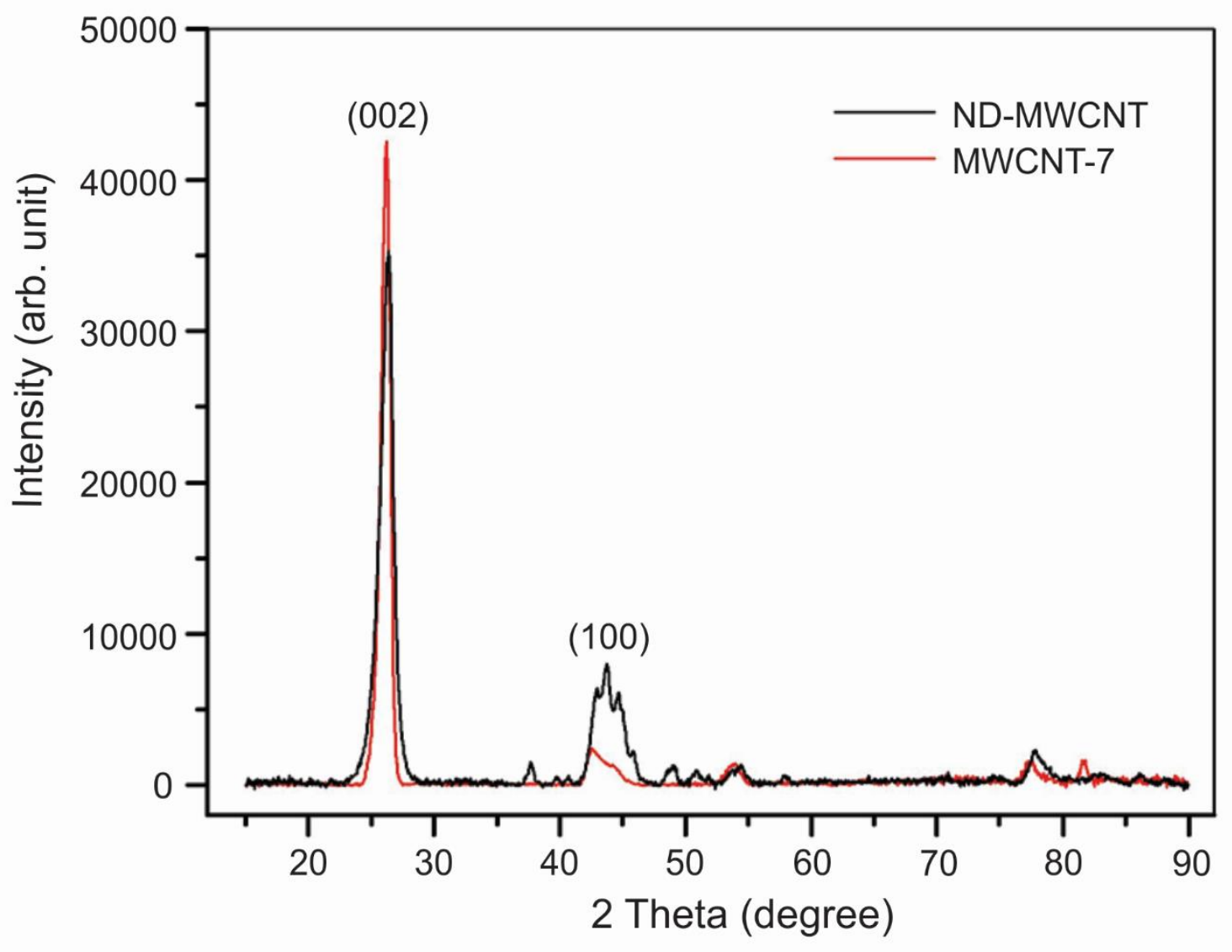

Figure 2.4: X-ray diffraction spectra of ND-MWCNT and MWCNT-7 samples. 


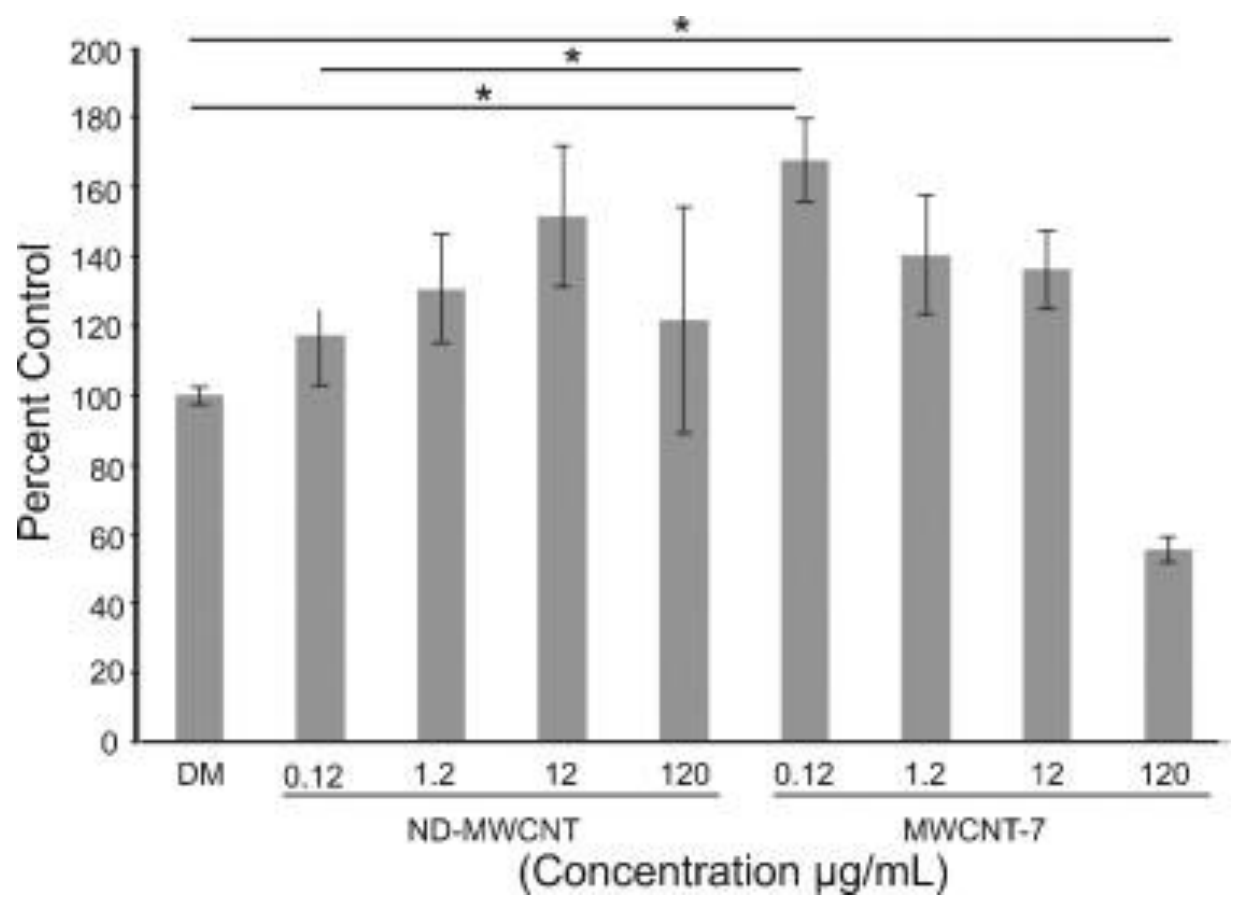

Figure 2.5: SAEC were treated with varying concentrations of DM, ND-MWCNT, or MWCNT-7 for a $24 \mathrm{~h}$ period. CellTiter $96^{\circledR}$ Aqueous One Solution was added $4 \mathrm{~h}$ prior to end of exposure and absorbance was measured at 490nm. Results indicate that MWCNT-7 induces a significant increase in cell proliferation at $0.12 \mu \mathrm{g} / \mathrm{ml}$ compared to DM and ND-MWCNT at $1.2 \mu \mathrm{g} / \mathrm{mL}$ and decrease at $120 \mu \mathrm{g} / \mathrm{mL}$ compared to $\mathrm{DM}(* p \leq 0.05)$. 

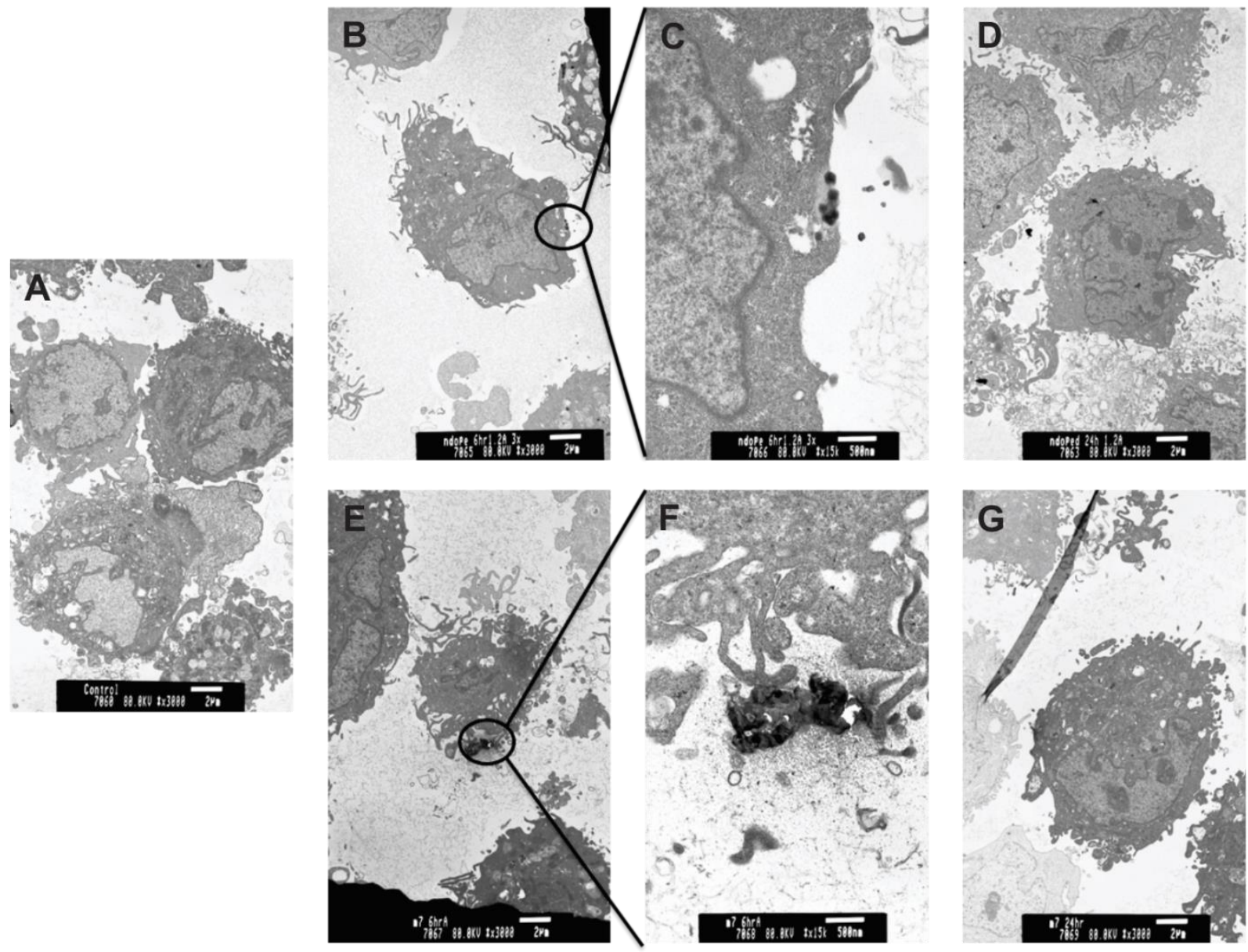

Figure 2.6: TEM images of SAEC treated with (A) $1.2 \mu \mathrm{g} / \mathrm{ml} \mathrm{DM,} \mathrm{(B,} \mathrm{C,} \mathrm{D)} \mathrm{ND-MWCNT,} \mathrm{or} \mathrm{(E,} \mathrm{F,} \mathrm{G)}$ MWCNT-7 for $6 \mathrm{~h}$ (column 2, 3) or $24 \mathrm{~h}$ (column 4). 


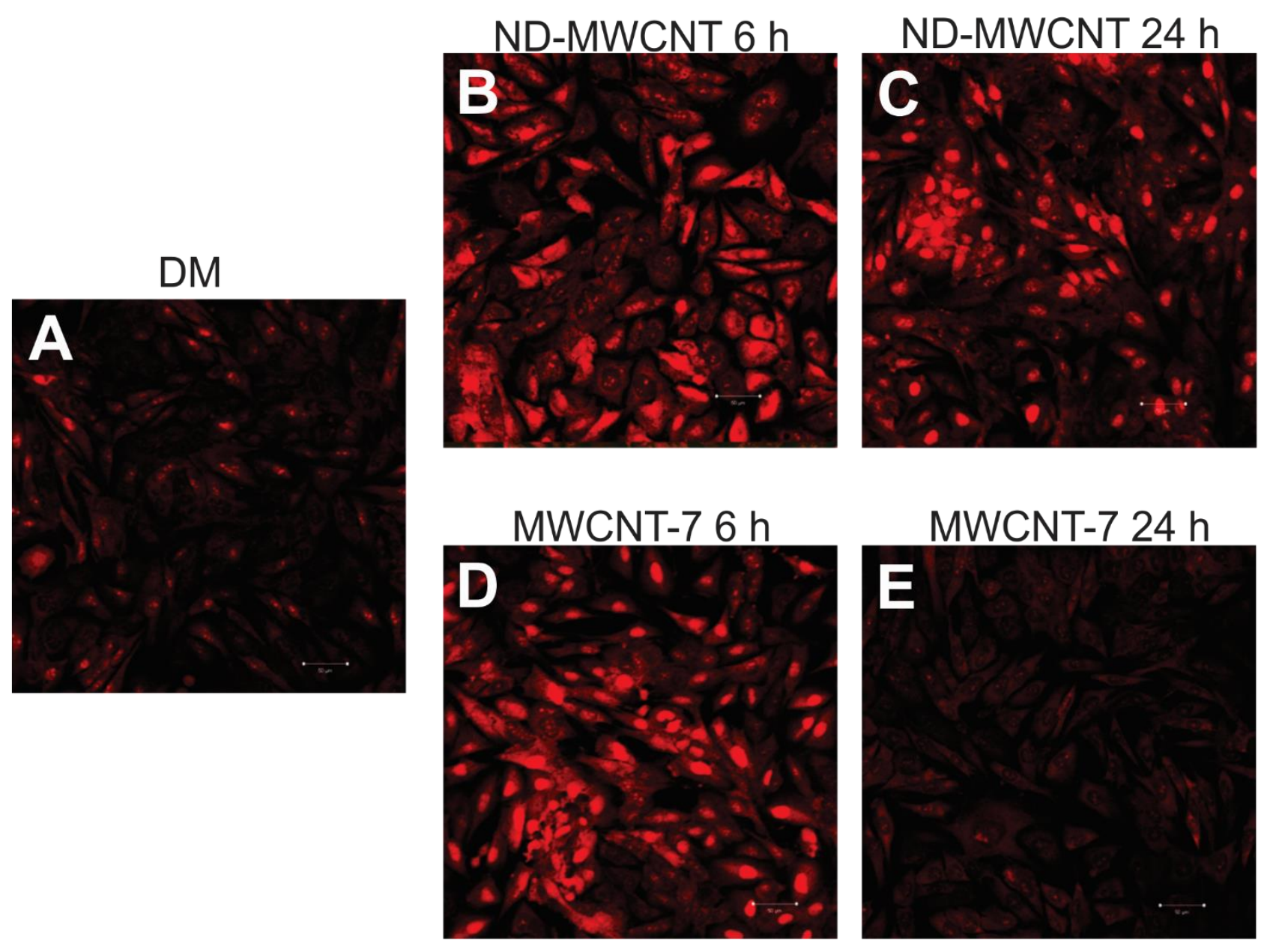

Figure 2.7: Confocal images of SAEC treated with $1.2 \mu \mathrm{g} / \mathrm{ml} \mathrm{DM} \mathrm{(A),} \mathrm{ND-MWCNT} \mathrm{(B,} \mathrm{C),} \mathrm{or} \mathrm{MWCNT-7} \mathrm{(D,}$ E) over $6(\mathrm{~B}, \mathrm{D})$ or $24 \mathrm{~h}(\mathrm{C}, \mathrm{E})$ followed by staining with DHE for presence of ROS. 

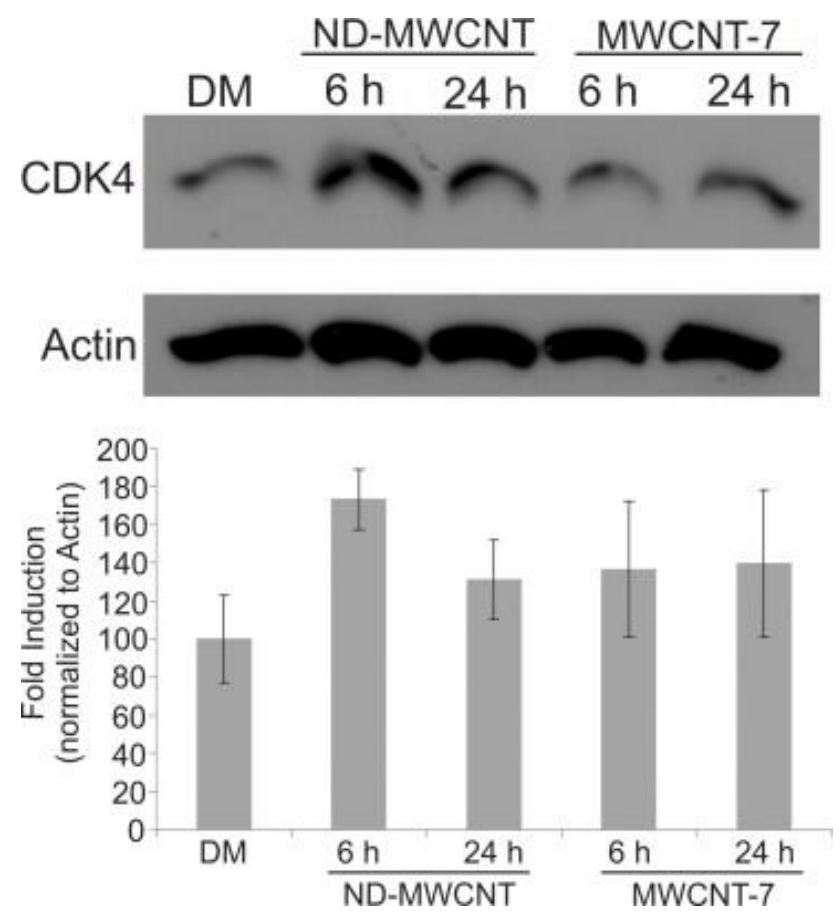

Figure 2.8: SAEC were grown to subconfluence and treated with $1.2 \mu \mathrm{g} / \mathrm{ml} \mathrm{DM}$, ND-MWCNT, or MWCNT-7 over 6 or $24 \mathrm{~h}$. Whole cell lysates were resolved by SDS-PAGE on ten percent gels and probed for CDK4, which appeared to be increased at $6 \mathrm{~h}$ in ND-MWCNT-treated SAEC. $\beta$-actin was used as a loading control. Densitometry results $(n=3)$ are presented below the blot. 

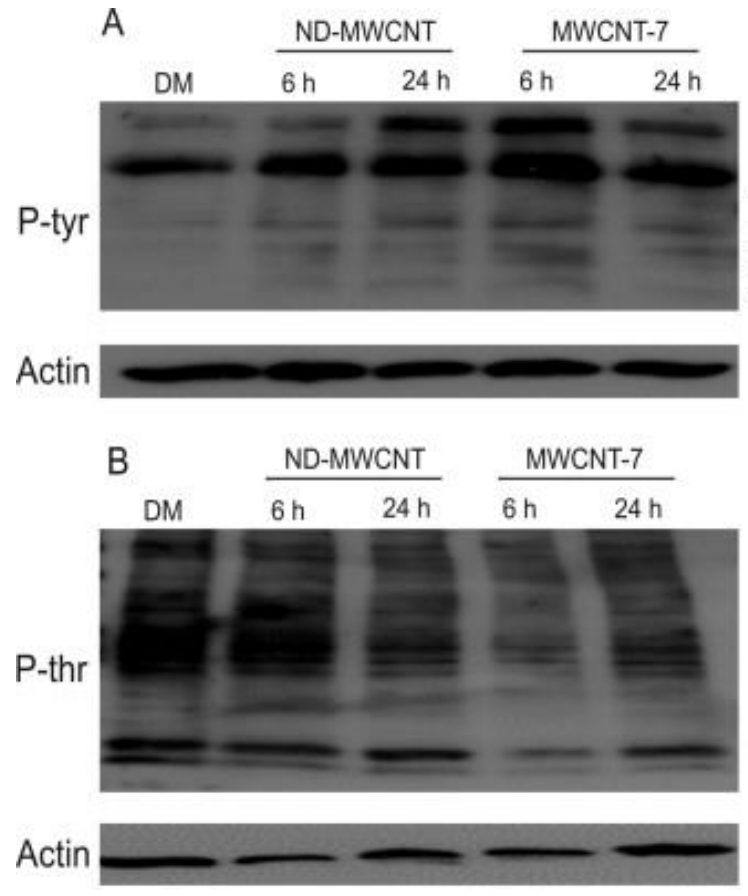
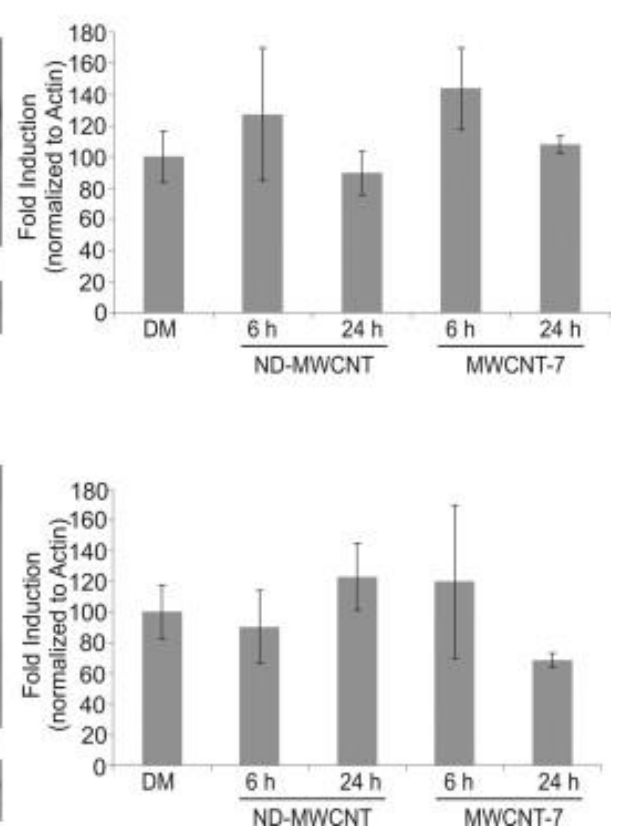

Figure 2.9: SAEC were treated with $1.2 \mu \mathrm{g} / \mathrm{ml}$ DM, ND-MWCNT, or MWCNT-7 over 6 or $24 \mathrm{~h}$. Whole cell lysates were resolved by SDS-PAGE on ten percent gels and probed for phospho-tyrosine (A) or phospho-threonine (B) indicating that cell signaling response may be time and particle dependent. Bactin was used as a loading control. Densitometry results $(n=3)$ are presented to the right of each blot. 
Table 2.1: Zeta potentials of ND-MWCNT and MWCNT-7 in PBS (pH 7.2) and serum-free media (pH 7.6)

\begin{tabular}{|l|l|l|l|l|}
\hline & Dimensions & & Zeta Potential $(\mathrm{mV})$ & (mean \pm standard error) \\
\hline ND-MWCNT & Length $(\mu \mathrm{m})$ & $5.28 \pm 2.07$ & PBS $(\mathrm{pH} 7.2)$ & $-13.9 \pm 0.483$ \\
\hline & Width $(\mathrm{nm})$ & $79.7 \pm 19.4$ & $\begin{array}{l}\text { Serum-free media }(\mathrm{pH} \\
7.6)\end{array}$ & $-12.9 \pm 0.835$ \\
\hline MWCNT-7 & Length $(\mu \mathrm{m})$ & $\begin{array}{l}3.86(\mathrm{GSD} \\
1.94)\end{array}$ & PBS $(\mathrm{pH} 7.2)$ & $-15.8 \pm 0.450$ \\
\hline & Width $(\mathrm{nm})$ & $49 \pm 13.4 \mathrm{~nm}$ & $\begin{array}{l}\text { Serum-free media }(\mathrm{pH} \\
7.6)\end{array}$ & $-12.2 \pm 0.283$ \\
\hline
\end{tabular}


Table 2.2: SAEC were grown to subconfluence and treated with $1.2 \mu \mathrm{g} / \mathrm{ml} \mathrm{DM}$, ND-MWCNT, or MWCNT7 over 6 or 24 h prior to ethanol fixation, staining with propidium iodide, and assessment using a FACS

SR instrument.. Results presented indicate an increased percentage of cells in G2 compared to DM and significant changes between 6 and $24 \mathrm{~h}$ in $\mathrm{G} 1$ and S phase in MWCNT-7 exposed SAEC, while NDMWCNT induced significant changes between 6 and $24 \mathrm{~h}$ in G2. Significant changes are also noted at 24 h between MWCNT-7 and ND-MWCNT exposed SAEC at G1 and S $(* p \leq 0.05)$.

\begin{tabular}{|c|c|c|c|}
\hline Treatment & \% G1 \pm std error & \% S \pm std error & \% G2 \pm std error \\
\hline MWCNT-7 DM & $79.3 \pm 2.03$ & $14.4 \pm 1.70$ & $3.37 \pm 0.232$ \\
\hline MWCNT-7 6 h & $\mathbf{7 7 . 8} \pm \mathbf{0 . 4 9 2 *}$ & $\mathbf{1 5 . 1} \pm \mathbf{0 . 3 2 5 *}$ & $\mathbf{4 . 2 5} \pm \mathbf{0 . 3 0 7 *}$ \\
\hline MWCNT-7 24 h & $\mathbf{7 9 . 6 \pm 0 . 5 8 7 *}$ & $\mathbf{1 4 . 2} \pm \mathbf{0 . 1 5 2 *}$ & $\mathbf{3 . 8 0} \pm \mathbf{0 . 3 4 4} *$ \\
\hline ND-MWCNT DM & $76.1 \pm 2.13$ & $16.1 \pm 1.9$ & $4.16 \pm 0.091$ \\
\hline ND-MWCNT 6 h & $79.1 \pm 2.59$ & $14.4 \pm 2.06$ & $\mathbf{3 . 4 5} \pm \mathbf{0 . 3 5 0 *}$ \\
\hline ND-MWCNT 24 h & $\mathbf{7 6 . 2} \pm \mathbf{0 . 8 4 8 *}$ & $16.6 \pm 0.848$ & $\mathbf{4 . 6 7} \pm \mathbf{0 . 2 2 2} *$ \\
\hline
\end{tabular}




\section{Chapter 3}

Effects of direct and indirect exposure to nitrogen-doped multi-walled carbon nanotubes and pristine multi-walled carbon nanotubes on lung fibroblasts 


\section{Abstract:}

It has previously been established that MWCNT-7 and ND-MWCNT induce pulmonary fibrosis in mice. ND-MWCNT were shown to induce less of an inflammatory and fibrotic response compared to MWCNT7 in vivo, but limited work has been carried out to support these data with a valid in vitro model that can be used for further assessment. Here, we compared the effects of indirect or direct exposure to NDMWCNT or MWCNT-7 on WI-38 fibroblasts over $48 \mathrm{~h}$ in monoculture by two distinct methods. WI-38 were either exposed to biologically relevant concentrations of interleukin-6 (IL-6) and interleukin-8 (IL-8) or by direct exposure to 0.0625 to $2.50 \mu \mathrm{g} / \mathrm{cm}^{2}$ ND-MWCNT or MWCNT-7. IL-6 and IL-8 concentrations were derived from a pro-inflammatory multiplex ELISA carried out on BEAS-2B conditioned media exposed to 1.25 or $2.50 \mu \mathrm{g} / \mathrm{cm}^{2}$ ND-MWCNT or MWCNT-7 over $24 \mathrm{~h}$ to assess an acute inflammatory response. A CFSE dye dilution proliferation assay suggested that MWCNT-7 associated concentrations of IL-6 and IL-6 plus IL-8 in combination as well as $.0 .250 \mu \mathrm{g} / \mathrm{cm}^{2}$ ND-MWCNT and $1.25 \mu \mathrm{g} / \mathrm{cm}^{2}$ of NDMWCNT or MWCNT-7 decreased cellular proliferation. Trypan Blue cell viability assays and total live cell counts further supported this conclusion. Collagen I production measured by western blot suggested a dose and particle-dependent response, with ND-MWCNT inducing more collagen I production at 0.0625 and $0.125 \mu \mathrm{g} / \mathrm{cm}^{2}$, while IL-6 and IL-8 treatment induced more subtle effects. Quantitative RT-PCR carried out on CDNA from ND-MWCNT and MWCNT-7 exposed WI-38 showed a similar ND-MWCNT induced response pattern for $\alpha$-SMA gene expression, suggesting a potential early stage transition towards myfibroblast activation. Overall, our results indicated that ND-MWCNT and MWCNT-7 induce effects in WI38 in a particle and dose-related manner, suggesting that the physicochemical characteristics of the materials may alter their biological effects, and that perhaps IL-6 and IL-8 are not primary drivers in pro-fibrogenic responses in WI-38. We also noted the limitations of the in vitro models and provide potential reasons for why ND-MWCNT appear to be more bioactive here. 


\section{Introduction:}

With increased development, production, and utilization of MWCNT and functionalized MWCNT in occupational settings and within consumer products, it has become progressively more important to determine the impact of these materials on the human body across a range of exposure doses and potential adverse outcomes. While many assessments have been carried out in vivo to assess the proinflammatory and fibrotic outcomes generally associated with MWCNT exposure in rodents, less is known on the relationship between toxicity and nitrogen doping of MWCNT in addition to the use of in vitro systems to accurately reflect these outcomes especially for complex multi-dimensional endpoints such as pulmonary fibrosis.

Pulmonary fibrosis is a group of disease states marked by progressive and irreversible destruction of lung tissues that lead to organ malfunction, disruption of gas exchange in the alveolar region, and ultimately, death by respiratory failure. The by-product of dysregulated wound repair, fibrosis leads to an overabundance of extracellular matrix deposition and other abnormal molecular changes that impact cell proliferation, cell death, and chronic cell signaling changes [144, 145]. It has been proposed that activated pulmonary epithelium at the sites of repetitive, on-going injury plays a key role in initializing dysregulated fibroblast proliferation and myofibroblast generation via paracrine signals. Myofibroblasts, which are activated contractile fibroblasts that express $\alpha$-SMA, disrupt the basement membrane underlying the epithelium and promote epithelial apoptosis, thus preventing normal re-epithelialization and wound repair in the fibrotic lung, followed by excessive ECM deposition and formation of scar tissue [146]. Many cytokines have clinically been associated with Idiopathic Pulmonary Fibrosis (IPF) and other fibrotic disorders such as IL-1, IL-4, IL-6, IL-8, IL-9, IL-13, monocyte chemoattractant protein-1 (MCP-1/CCL2), tumor necrosis factor (TNF), transforming growth factors (TGF), and platelet-derived growth factor (PDGF) $[147,148]$. A recent study of MWCNT-7 induced fibrosis in mice showed increased TNF- $\alpha$, IL-1, IL-6, and CCL2 in BAL fluid, as well as the presence of TGF $\beta$ 
and PDGF-A in lung tissue, which builds upon the work of Porter et al. and Mercer et al. that first identified an inflammatory and fibrotic response to MWCNT-7 several years ago [40, 76, 77]. However, it is unknown as to which cell type was primarily responsible for producing the cell signaling molecules, nor is it simple to study complex cell-cell communication in vivo with commonly utilized techniques. In vitro studies, which may be better suited to the study of specific cell signaling processes within and between cell types, have suggested that direct exposure of fibroblasts to pristine variants of MWCNT induce a length-dependent response including increased fibroblast proliferation, differentiation into activated myofibroblasts, and increased collagen production $[41,110,149,150]$. Several in vitro studies have begun addressing the responses of epithelial cells as well, which are physiologically relevant to the process of fibrogenesis and MWCNT exposure $[41,110]$. Following macrophage, epithelial cells within the lung are the primary defense between the outside world and inhaled particles, typically excluding foreign material from the interstitial space [151]. However, it has been shown in vivo that MWCNT can traverse the epithelial barrier by directly piercing the cells dependent upon the CNT stiffness and crystallinity $[105,152]$ are endocytosed as agglomerates, or are carried across the epithelium within macrophage [105], after which they are likely free to interact with resident fibroblasts and other cell types underlying the epithelium, thus providing the impetus to treat fibroblasts directly in vitro.

Not as much research has been carried out on the distribution, uptake, and fibrotic bioactivity of ND-MWCNT. Others have shown that nitrogen-functionalized MWCNT decrease BEAS-2B viability and increase DNA damage compared to pristine MWCNT at moderate exposure doses [66], and increase or induce similar release of soluble IL-1 $\beta$, PDGF-AA, and TGF $\beta$ compared to control or pristine MWCNT, respectively, suggesting that ND-MWCNT may not significantly decrease overall toxicity [68]. Conversely, an in vivo study assessing ND-MWCNT by intratracheal instillation in rats suggested that NDMWCNT could be fibrotic, but only at very high doses, which provided limited information on the fibrotic potential of ND-MWCNT at occupationally relevant exposures [70]. However, an unpublished in vivo 
mouse study using the same ND-MWCNT used in this study at occupational exposure doses suggested decreased inflammatory and fibrotic compared to MWCNT-7, also suggesting that they may be less

bioactive. In addition to surface chemistry properties that may significantly impact their toxicity, uptake into macrophage and other cell types, and reactivity based upon particle surface area, biopersistence also plays an important role in understanding the long-term effects of ND-MWCNT exposure. It has been shown ex vivo that short ND-MWCNT can be broken down completely within 80 days layer by layer in the presence of a biologically relevant concentration of $\mathrm{H}_{2} \mathrm{O}_{2}$ found within cellular lysosomes due to the presence of nitrogen-induced defects in the carbon lattice [153].

Based on previous in vivo studies that have shown MWCNT-7 and ND-MWCNT-induced fibrogenesis, and the aforementioned interaction with epithelial and fibroblast cells, we hypothesized that the physicochemical properties of these particles would elicit differing responses in two independent in vitro systems utilizing BEAS-2B and WI-38. We assessed specific paracrine signaling between BEAS-2B and WI-38 by supplying CNT-induced epithelial-derived IL- 6 and IL- 8 at biologicallyrelevant concentrations as well as the impact of exposing WI-38 to ND-MWCNT and MWCNT-7 directly. WI-38 were assessed by determining changes in cellular proliferation, collagen I production, and gene expression suggesting early fibroblast to myofibroblast transition.

\section{Materials and Methods:}

\subsection{ND-MWCNT and MWCNT-7}

The ND-MWCNT used in this study were a gift from Mauricio Terrones (Pennsylvania State University, University Park, PA), and Morinobu Endo, and Shuji Tsuruoka (Shinshu University, Nagano, Japan). NDMWCNT were characterized at the Morgantown National Institute for Occupational Safety and Health and West Virginia University Shared Research Facilities. The MWCNT-7 used in this study were originally obtained through the Mitsui \& Co., Ltd. (MWCNT-7, lot \#05072001K28) and previously characterized (Porter et al. 2010). 


\subsection{ND-MWCNT and MWCNT-7 preparation}

For cell culture studies, ND-MWCNT were prepared in dispersion media (DM) consisting of PBS (pH 7.4, $\mathrm{Ca} / \mathrm{Mg}$-free) supplemented with $0.6 \mathrm{mg} / \mathrm{ml}$ mouse serum albumin and $0.01 \mathrm{mg} / \mathrm{ml}$ 1,2-dipalmitoyl-snglycero-3-phosphocholine (DPPC) as previously described [118]. DPPC was prepared fresh as a $1 \mathrm{mg} / \mathrm{ml}$ stock solution in $100 \%$ ethanol. Transmission electron microscopy (TEM) micrographs of MWCNT dispersed in DM demonstrated that DM promotes significant dispersion of MWCNT [118]. ND-MWCNT and MWCNT-7 were prepared in DM by indirect sonication at $4^{\circ} \mathrm{C}$ for 5 min (Hielscher ultrasonic processor, UIS259L; Ringwood, NJ) at amplitude 100\% and cycle 1. Following indirect sonication, the suspension was directly sonicated for 5 min at $5 \mathrm{~W}$ output and $30 \%$ duty cycle in 1 min increments (Branson Sonifier 450; Danbury, CT). The stock solution $(0.5 \mathrm{mg} / \mathrm{ml}$ ) of ND-MWCNT or MWCNT-7 was kept at $4^{\circ} \mathrm{C}$ and used within 2-3 weeks. The ND-MWCNT and MWCNT-7 stock solutions were directly sonicated for $1 \mathrm{~min}$ at $5 \mathrm{~W}$ output and $30 \%$ duty cycle prior to cell culture experiments. Cells were treated with $1.25 \mu \mathrm{g} / \mathrm{cm}^{2}$ or $2.50 \mu \mathrm{g} / \mathrm{cm}^{2} \mathrm{DM}$, ND-MWCNT, or MWCNT-7.

\subsection{Cell Culture}

BEAS-2B bronchial epithelial cells were purchased from ATCC and maintained in DMEM: F-12 (ATCC, Manassas, VA) with 10\% FBS (Atlanta Biologicals, Norcross, GA) and 5\% percent penicillin-streptomycin (Lonza, Walkersville, MD), and all experiments were carried out using cells below passage 15 . WI-38 normal human lung fibroblasts were purchased from ATCC and maintained in EMEM (ATCC) with 10\% FBS (Atlanta Biologicals) and 5\% penicillin-streptomycin (Lonza), and all experiments were carried out using cells below passage 9 . All cell types were maintained in an incubator at $37^{\circ} \mathrm{C}$ with $5 \% \mathrm{CO}_{2}$. Recombinant IL-6 and IL-8 were purchased from R\&D Systems (Minneapolis, MN) and added in biologically-relevant concentrations to reflect IL- 6 and IL-8 produced by BEAS-2B that were treated with $1.25 \mu \mathrm{g} / \mathrm{cm}^{2}$ or $2.50 \mu \mathrm{g} / \mathrm{cm}^{2}$ ND-MWCNT or MWCNT-7 as assessed by a pro-inflammatory multiplex. IL13 was excluded from further analysis as its $\mathrm{pg} / \mathrm{mL}$ concentration in BEAS-2B media was extremely low 
and available recombinant IL-13 was shown to induce biological effects only at concentrations nearly 100-fold higher.

\subsection{Transmission Electron Microscopy}

BEAS-2B interaction with and engulfment of ND-MWCNT and MWCNT-7 were analyzed by TEM. BEAS2B were grown to confluence and exposed to $1.25 \mu \mathrm{g} / \mathrm{cm}^{2}$ or $2.50 \mu \mathrm{g} / \mathrm{cm}^{2} \mathrm{DM}$, ND-MWCNT, or MWCNT7 for 24 h. Additionally, WI-38 interaction with and engulfment of ND-MWCNT and MWCNT-7 were analyzed by TEM. WI-38 were grown to confluence and exposed to $0.0625,0.125,0.250,1.25$, or 2.50 $\mu \mathrm{g} / \mathrm{cm}^{2}$ DM, ND-MWCNT, or MWCNT-7 for $48 \mathrm{~h}$. Cells were trypsinized with Trypsin-EDTA (Gibco, Waltham, MA) per manufacturer guidelines and harvested by centrifugation at $400 \times \mathrm{g}$ for $5 \mathrm{~min}$. Cells were fixed in Karnovksy's fixative (2.5\% glutaraldehyde and 3\% paraformaldehyde in $0.1 \mathrm{M}$ sodium cacodylate, $\mathrm{pH} 7.4$ ), and $4 \%$ agarose was added to the pellet and lightly agitated such that agarose surrounded the pellet. Excess solid agarose was trimmed away and placed in fresh Karnovsky's fixative over a day. Pellets were post-fixed in $1 \%$ osmium tetraoxide, and stained with $1 \%$ tannic acid and $0.5 \%$ uranyl acetate. The cells were dehydrated by sequential washings in $50,70,90$, and $100 \%$ ethanol and embedded in LX-112 (Ladd; Williston, VT). Ultrathin sections $70 \mathrm{~nm}$ thick were cut using a Leica UC7 ultramicrotome, placed on copper mesh grids, stained with $4 \%$ uranyl acetate ad lead citrate, and examined with a JEOL JEM-1400 transmission electron microscope (Peabody, MA). Images were taken using a mid-mount CCD digital camera system (Advanced Microscopy Techniques Corporation XR81M-B camera, Woburn, MA).

\subsection{Cell viability and Total Live Cell Counting}

WI-38 were plated in six well dishes and exposed to biologically-relevant and manufacturer's suggested concentrations of IL-6, IL-8, or IL-6 + IL-8 or exposed to $0.0625,0.125,0.250$, or $1.25 \mu \mathrm{g} / \mathrm{cm}^{2} \mathrm{DM}$, NDMWCNT, or MWCNT-7 for $48 \mathrm{~h}$. At the end of the exposure, WI-38 were trypsinized, centrifuged at 400xg 5 minutes, resuspended in PBS, and kept on ice while assessing all of the samples. A Countess 
Automated Cell Counter (Invitrogen, Carlsbad, CA) and manufacturer supplied Trypan Blue were used to assess total live cell counts and percent viability for three independent experiments. Treatment groups were compared by One Way ANOVA analysis and Tukey's Multiple Comparison test.

\subsection{Meso Scale Discovery Pro-inflammatory Cytokine Kit}

A Meso Scale Discovery V-PLEX Human Pro-inflammatory Panel 1 Kit was used to assess conditioned media for IFN- $\Upsilon$, IL-1 $\beta$, IL-2, IL-4, IL-6, IL-8, IL-10, IL-12p70, IL-13, and TNF- $\alpha$ from BEAS-2B treated $24 \mathrm{~h}$ with $1.25 \mu \mathrm{g} / \mathrm{cm}^{2}$ or $2.50 \mu \mathrm{g} / \mathrm{cm}^{2}$ DM, ND-MWCNT, or MWCNT-7 in 48 well plate from three independent experiments. Media was collected, frozen at $-80^{\circ} \mathrm{C}$, thawed on ice, and centrifuged to pellet excess CNT and debris. Manufacturer's protocol was followed with each independent sample plated in technical duplicate, and the plate was read on an MSD Sector Imager 2400.

\subsection{Dose response proliferation assay using CellTiter 96 Aqueous One Solution}

A dose-response assessment of cellular proliferation was carried out by seeding WI-38 in 96-well plates for $24 \mathrm{~h}$, followed by exposure to DM, ND-MWCNT, or MWCNT-7 for $48 \mathrm{~h}$ at $0.125,0.250,1.25$, or 2.50 $\mu \mathrm{g} / \mathrm{cm}^{2}$. Twenty microliters of CellTiter $96^{\circledR}$ Aqueous One Solution (Promega; Madison, WI) was added to each well during the last 2 hours of exposure. Absorbance was read at $490 \mathrm{~nm}$ using a BioTek Synergy H1 plate reader (Winooski, VT), and statistical analyses were carried out using GraphPad Prism (Redmond, WA). Three independent experiments were performed; treatment groups were averaged by technical triplicate and compared by One Way ANOVA analysis and Tukey's Multiple Comparison test.

\subsection{Cell Proliferation}

A CellTrace CFSE Cell Proliferation Kit (Life Technologies, Carlsbad, CA) was utilized along with a LIVE/DEAD Fixable Violet Dead Cell Stain Kit (Life Technologies, Carlsbad, CA) to assess cell proliferation in WI-38 exposed to various concentrations of IL- 6 , IL-8, or IL- $6+$ IL-8 or $0.062,0.125,0.250$, and 1.25 $\mu \mathrm{g} / \mathrm{cm}^{2} 48 \mathrm{~h}$. $60 \mathrm{~mm}$ dishes were used for these experiments. Cells were labeled with CFSE according to manufacturer's protocol, and time 0 was taken 30 minutes after plating cells, and an additional time 0 
was taken upon cell exposure $24 \mathrm{~h}$ later and used for analyses. At the end of the total exposure times described above, cells were trypsinized, assessed for viability and total number of live cells using Trypan Blue as described above, and stained using Violet LIVE/DEAD staining per manufacturer's protocol. Cells were fixed in $4 \%$ paraformaldehyde for 10 minutes, washed twice with PBS+1\%BSA, resuspended, and stored at $4^{\circ} \mathrm{C}$ until read on a BD LSRII Flow Cytometer (Franklin Lakes, NJ) utilizing BD FACSDiva Software. $488 \mathrm{~nm}$ and $405 \mathrm{~nm}$ lasers were utilized; CFSE had an excitation wavelength of $492 \mathrm{~nm}$ and emission wavelength of $517 \mathrm{~nm}$ and LIVE/DEAD had an excitation wavelength of $416 \mathrm{~nm}$ and emission wavelength of $451 \mathrm{~nm}$. Preliminary experiments also indicated that compensation was not needed for these dyes. 10,000 events were collected per individual sample three independent biological experiments. Cells were gated using forward and side scatter analysis of unstained cells, as well as for singlets. Single dye controls were used to set gating for dead cells and laser settings for CFSE (cell aliquot collected 20 minutes after initial labeling with CFSE). Data were analyzed based upon the percentage of cells remaining in time 0 after the full exposure time, FITC median (which correlates with the CFSE dye intensity per sample), and percentage of cells dead. Median intensity values were determined using FlowJo Software (Ashland, OR), and analyzed by One Way ANOVA and Tukey's Multiple Comparison test.

\subsection{Western blotting}

WI-38 were exposed to various concentrations of IL-6, IL-8, or IL-6 + IL-8 or $0.062,0.125,0.250$, and 1.25 $\mu \mathrm{g} / \mathrm{cm}^{2} 48 \mathrm{~h}$ prior to making lysates. Whole cell protein extraction was carried out using RIPA buffer (150 mM NaCl, 10 mM Tris pH 7.4, 2 mM EDTA, 1\% IGEPAL, 1\% sodium deoxycholate, 0.1\% sodium dodecyl sulfate) supplemented with a $10 \mu \mathrm{g} / \mathrm{ml}$ protease inhibitor cocktail and $10 \mu \mathrm{g} / \mathrm{ml}$ phosphatase inhibitor (Thermo Fisher Scientific; Waltham, MA). Protein concentration was measured using a BCA protein assay kit (Thermo Fisher Scientific, Waltham, MA). Twenty micrograms of protein per sample was resolved using sodium dodecyl sulfate-polyacrylamide gel electrophoresis (SDS-PAGE) and 
transferred to polyvinylidene fluoride membranes (PVDF, Pall Corporation; Pensacola, FL). Membranes were blocked for $1 \mathrm{~h}$ in 5\% milk in Tris Buffered Saline with Tween-20 (TBST) (62.5mM Tris pH 7.4, $150 \mathrm{mM} \mathrm{NaCl}, 0.05 \%$ Tween 20) (Thermo Fisher Scientific, Waltham, MA) and incubated with Collagen I antibody (Fitzgerald, Acton, MA) or $\beta$-actin (Sigma-Aldrich, St. Louis, MO) primary antibodies overnight at $4^{\circ} \mathrm{C}$. Membranes were washed three times with TBST for ten min each and incubated with speciesspecific secondary antibodies for $1 \mathrm{~h}$ at room temperature. Analysis was carried out using ECL Western Blotting Substrate (Thermo Fisher Scientific, Waltham, MA). Representative western blots are from three independent experiments per antibody.

\subsection{Quantitative Real-Time PCR}

RNA was isolated from WI-38 using a Qiagen RNeasy Mini Kit according to the manufacturer's protocol (Qiagen, Hilden, Germany), and concentration was determined using a NanoDrop 1000 Spectrophotometer (NanoDrop Technologies, Wilmington, DE). 1 g of RNA per sample was converted into complementary DNA (cDNA) using a High Capacity cDNA Reverse Transcription Kit from Applied Biosystems (Life Technologies, Carlsbad, CA). The manufacturer's suggested thermal cycling conditions were used as follows: $25^{\circ} \mathrm{C}$ for 10 minutes, 2 cycles of $37^{\circ} \mathrm{C}$ for 60 minutes, and $85^{\circ} \mathrm{C}$ for 5 seconds, and held at $4^{\circ} \mathrm{C}$. Quantitative real-time PCR reactions were performed using an Applied Biosystems 7500 Real-Time PCR instrument. As per manufacturer guidelines, cDNA was diluted 1:10 in nuclease free water and mixed with TaqMan Gene Expression Assays diluted 1:10 in TaqMan Universal PCR Master Mix, all from Applied Biosystems (Fosters City, CA). Gene expression assay numbers utilized are provided as follows: 18S (Hs99999901_s1), ACT2A (Hs00426835_g1), and COL1A1 (Hs00164004_m1). qRT-PCR analysis for each sample was carried out in duplicate and normalized to the $18 \mathrm{~S}$ housekeeping gene. Thermal cycling conditions were as follows: $50^{\circ} \mathrm{C}$ for $2 \mathrm{~min}, 95^{\circ} \mathrm{C}$ for $10 \mathrm{~min}$, followed by 40 cycles of $95^{\circ} \mathrm{C}$ for $15 \mathrm{~s}$ and $60^{\circ} \mathrm{C}$ for $10 \mathrm{~min}$. Fold change was determined using the $2^{-\Delta \Delta \mathrm{ct}}$ method. 


\subsection{Statistical Analysis}

Statistical analyses for the data were performed using GraphPad Prism 7.00. One Way ANOVA tests comparing across all means for sample groups or between the control only and exposure groups were utilized, and Tukey's Multiple Comparison's Test or Dunnett's's test, respectively, were used to determine significance between treatment groups.

Results:

3.1 TEM assessment suggests that BEAS-2B interact with ND-MWCNT and MWCNT-7. BEAS-2B were exposed to 1.25 or $2.50 \mu \mathrm{g} / \mathrm{cm}^{2}$ DM, ND-MWCNT or MWCNT-7 for $24 \mathrm{~h}$ and prepared for assessment by TEM. CNT were primarily found in bundles or agglomerates that primarily appeared to be endocytosed (Figure 3.1 C, D; Figure 3.2 C, D, H). ND-MWCNT were also observed piercing the BEAS-2B, providing support that MWCNT are taken up into cells by multiple pathways regardless of functionalization in this case.

\subsection{ND-MWCNT and MWCNT-7 induce particle and dose-dependent changes in BEAS-2B viability.}

BEAS-2B were assessed for cell viability and number of total live cells using Trypan Blue and a Countess Automated Cell Counter after $24 \mathrm{~h}$ exposure to 1.25 or $2.50 \mu \mathrm{g} / \mathrm{cm}^{2} \mathrm{DM}$, ND-MWCNT, or MWCNT-7. Results from three experiments were normalized to percent control, and a one-way ANOVA test, followed by Tukey's post hoc analysis comparing means from all treatment groups, was carried out. Results suggest that BEAS-2B exposed to $2.50 \mu \mathrm{g} / \mathrm{cm}^{2} \mathrm{ND}-\mathrm{MWCNT}$ were less viable when compared to DM control $(p<0.0089)$ or cells treated with $1.25 \mu \mathrm{g} / \mathrm{cm}^{2}$ MWCNT-7 $(p<0.0027)$, suggesting that both dose and particle may significantly impact BEAS-2B viability. A significant difference in viability was also observed by comparison of BEAS-2B exposed to $2.50 \mu \mathrm{g} / \mathrm{cm}^{2}$ ND-MWCNT or $2.50 \mu \mathrm{g} / \mathrm{cm}^{2}$ MWCNT-7 $(\mathrm{p}<$ 0.0442) (Figure 3.3 A). BEAS-2B were simultaneously counted for total number of live; results suggested that there was no significant change between any exposures (Figure 3.3 B). 
3.3 ND-MWCNT and MWCNT-7 induce pro-inflammatory cytokines in BEAS-2B in a dose and particledependent manner

In order to characterize ND-MWCNT and MWCNT-7 induced pro-inflammatory responses in BEAS-2B exposed to 1.25 or $2.50 \mu \mathrm{g} / \mathrm{cm}^{2}$ DM, ND-MWCNT, or MWCNT-7, a Meso Scale Discovery Human V-PLEX Pro-inflammatory Panel Kit was used to assess production of 10 cytokines and chemokines present in conditioned media. After $24 \mathrm{~h}$ exposure, BEAS-2B conditioned media had significantly elevated levels of IL-13, IL-6, and IL-8. IL-13 was shown to be significantly elevated to $4.29 \mathrm{pg} / \mathrm{mL}$ in BEAS-2B exposed to $2.50 \mu \mathrm{g} / \mathrm{cm}^{2}$ MWCNT-7 compared to control at $1.68 \mathrm{pg} / \mathrm{mL}(\mathrm{p}<0.0282)$ (Figure 3.4 A). IL-6 was shown to be significantly elevated to $44.9 \mathrm{pg} / \mathrm{mL}$ in cells again exposed to $2.50 \mu \mathrm{g} / \mathrm{cm}^{2} \mathrm{MWCNT}-7$ compared to control at $21.1 \mathrm{pg} / \mathrm{mL}(\mathrm{p}<0.0011)$. Additionally, IL-6 was significantly increased between BEAS-2B exposed to $1.25 \mu \mathrm{g} / \mathrm{cm}^{2}$ ND-MWCNT compared to $2.50 \mu \mathrm{g} / \mathrm{cm}^{2}$ MWCNT-7 $(\mathrm{p}<0.0002)$, suggesting that IL-6 production may be particle-related. A significant increase was also noted between cells exposed to $1.25 \mu \mathrm{g} / \mathrm{cm}^{2}$ and $2.50 \mu \mathrm{g} / \mathrm{cm}^{2}$ MWCNT-7 ( $<$.0218), suggesting that IL-6 production may also be doserelated by particle (Figure 3.4 B). IL-8 appeared to have the most pronounced activity in conditioned media samples from cells treated with ND-MWCNT or MWCNT-7. BEAS-2B exposed to $2.50 \mathrm{\mu g} / \mathrm{cm}^{2} \mathrm{ND}-$ MWCNT and MWCNT-7 produced $772.8 \mathrm{pg} / \mathrm{mL}$ and $944.9 \mathrm{pg} / \mathrm{mL}$ of IL-8, respectively, compared to control at $497.3 \mathrm{pg} / \mathrm{mL}(\mathrm{p}<0.0016 ; \mathrm{p}<0.0001)$. Differences were also observed between cells exposed to 1.25 and $2.50 \mu \mathrm{g} / \mathrm{cm}^{2}$ ND-MWCNT ( $<$ < 0.0005), $1.25 \mu \mathrm{g} / \mathrm{cm}^{2}$ ND-MWCNT and $2.50 \mu \mathrm{g} / \mathrm{cm}^{2}$ MWCNT-7 ( $p<0.0001), 1.25 \mu \mathrm{g} / \mathrm{cm}^{2}$ and $2.50 \mu \mathrm{g} / \mathrm{cm}^{2}$ MWCNT-7 $(p<0.0001)$, and $1.25 \mu \mathrm{g} / \mathrm{cm}^{2}$ MWCNT-7 and 2.50 $\mu \mathrm{g} / \mathrm{cm}^{2}$ ND-MWCNT $(\mathrm{p}<0.0402)$, again suggesting a dose and particle-dependent effect on cytokine production (Figure $\mathbf{3 . 4} \mathrm{C}$ ).

\subsection{Experimental design addresses two possible exposure scenarios in vitro.}

In order to address the indirect effects of epithelial-derived cell signaling molecules on fibroblast activity compared to the impact of direct exposure to ND-MWCNT or MWCNT-7, parallel experiments were 
carried out to monitor WI-38 proliferation and collagen I production (Figure 3.5.). We were interested in studying paracrine signaling between epithelial and fibroblast cells, which has been investigated in IPF in vitro models and patients [154]. A co-culture approach and conditioned media approach using media directly from exposed BEAS-2B was initially attempted (see Appendix 2), but rejected in favor of using recombinant IL-6 and IL-8 to study effects on WI-38, thus minimizing variability between experiments. Direct exposure of WI-38 over a dose range from 0.0625 to $2.50 \mu \mathrm{g} / \mathrm{cm}^{2}$ ND-MWCNT or MWCNT-7 was also utilized. Both experimental designs were rooted in in vivo observations that MWCNT can penetrate and pass through the alveolar epithelium to the interstitial space where resident fibroblasts are located soon after exposure. In addition, it has been shown that MWCNT-7 remain within the overall lung burden and is slowly released to the epithelial layer and interstitial space over extended periods of time [78].

\subsection{TEM assessment suggests that ND-MWCNT and MWCNT-7 interact with WI-38.}

WI-38 were exposed to $0.125,0.250,1.25$, or $2.50 \mu \mathrm{g} / \mathrm{cm}^{2} \mathrm{DM}$, ND-MWCNT or MWCNT-7 for $48 \mathrm{~h}$ and prepared for assessment by TEM to address particle-cell interactions and better understand how fibroblasts may take up the particles in question. CNT were observed interacting with the cells in two primary ways: engulfment and piercing. Areas of the micrographs where white "holes" are apparent were likely to be sites of CNT penetration observed in cells exposed to $0.125 \mu \mathrm{g} / \mathrm{cm}^{2}$ MWCNT-7 (Figure 3.6. D, E), $0.250 \mu \mathrm{g} / \mathrm{cm}^{2}$ MWCNT-7 (Figure 3.6. H, I), $1.25 \mu \mathrm{g} / \mathrm{cm}^{2}$ MWCNT-7 (Figure 3.6. L, M), 2.50 $\mu \mathrm{g} / \mathrm{cm}^{2}$ MWCNT-7 (Figure 3.6. P, Q) and $1.25 \mu \mathrm{g} / \mathrm{cm}^{2}$ ND-MWCNT (Figure 3.6. J, K). Generally, NDMWCNT appeared to be found in smaller agglomerates or isometric forms (Figure 3.6. G, 0), while MWCNT-7 were found as singlets, bundles, and diffuse agglomerates (Figure 3.6. E, I, M).

\subsection{Direct exposure of WI-38 to ND-MWCNT and MWCNT-7 are cytotoxic at high exposures.}

An MTS assay was carried out to determine the effects of ND-MWCNT and MWCNT-7 on WI-38 toxicity and establish a dose range for further study. WI-38 were exposed to $0.125,0.250,1.25$, or $2.50 \mu \mathrm{g} / \mathrm{cm}^{2}$ 
DM, ND-MWCNT or MWCNT-7 for $48 \mathrm{~h}$. WI-38 treated with $2.50 \mu \mathrm{g} / \mathrm{cm}^{2}$ ND-MWCNT exhibited significant cytotoxicity $(p<0.0195)$ and this dose was subsequently removed from further study since our aims were to assess the fibrogenic potential of CNT and not cytotoxicity resulting in death (Figure 3.7). No significant difference was observed between particles within the same dose. Significant differences were also observed between $0.125 \mu \mathrm{g} / \mathrm{cm}^{2}$ ND-MWCNT and $2.50 \mu \mathrm{g} / \mathrm{cm}^{2}$ ND-MWCNT or MWCNT-7 ( $p<0.0009 ; p<0.0107), 0.125 \mu \mathrm{g} / \mathrm{cm}^{2}$ MWCNT-7 and $2.50 \mu \mathrm{g} / \mathrm{cm}^{2}$ ND-MWCNT or MWCNT-7 $(\mathrm{p}<0.0021, \mathrm{p}<0.0241), 0.250 \mu \mathrm{g} / \mathrm{cm}^{2}$ ND-MWCNT and $2.50 \mu \mathrm{g} / \mathrm{cm}^{2}$ ND-MWCNT or MWCNT-7 $(\mathrm{p}<$ 0.0039; $\mathrm{p}<0.0418$ ), and 0.250 MWCNT-7 and $2.50 \mu \mathrm{g} / \mathrm{cm}^{2}$ ND-MWCNT or MWCNT-7 $(\mathrm{p}<0.0044 ; \mathrm{p}<$ 0.0469), all suggesting that the highest dose of ND-MWCNT and MWCNT-7 was cytotoxic across all doses assessed (Table 3.1).

\subsection{Recombinant IL-6 and IL-8 at biologically relevant concentrations nor direct exposure to ND-} MWCNT or MWCNT-7 induce effects on WI-38 viability and total cell counts.

To determine the impact of epithelial-derived pro-inflammatory cytokine production on fibroblasts, WI38 were exposed to recombinant IL-6, IL-8, and IL- 6 and 8 in combination in concentrations derived from data presented in Figure 3.4. To proximate biologically relative concentrations, $\mathrm{pg} / \mathrm{mL}$ values for IL-6 and IL-8 from BEAS-2B exposed to $2.50 \mu \mathrm{g} / \mathrm{cm}^{2}$ ND-MWCNT or MWCNT-7 were converted into $\mathrm{ng} / \mathrm{mL}$ concentrations that could be administered to WI-38 directly. WI-38 were exposed to 0.03 (IL-6 “ND"), 0.045 (IL-6 “M7”), and $0.1 \mathrm{ng} / \mathrm{mL}$ (manufacturer's suggestion) of IL-6, 0.77 (IL-8 “ND”), 0.94 (IL-8 “M7”), and $2.0 \mathrm{ng} / \mathrm{mL}$ (manufacturer's suggestion) of IL-8. Combination exposures matched IL-6 and IL-8 concentrations per particle or manufacturer's suggestion. Trypan Blue and a Countess Automated Cell Counter were utilized to determine if IL- 6 or IL-8 exposure had an impact on cell viability or total cell counts. Treatment with IL-6 or IL-8 over 48 hours were shown to have no effect upon cell viability or total live cell counts compared to control and between groups (Figure 3.9 A, B). 
Based upon results presented in Figure 3.7, a lower dose of $0.0625 \mu \mathrm{g} / \mathrm{cm}^{2}$ was added for assessment through the rest of the studies, as higher doses appeared to be cytotoxic. Doses below $0.0625 \mu \mathrm{g} / \mathrm{cm}^{2}$ were not assessed, although others have done so [110]. An exposure of $0.0625 \mu \mathrm{g} / \mathrm{cm}^{2}$ was roughly equivalent to a $30 \mu \mathrm{g}$ dose of MWCNT in mice based upon the alveolar surface area which is quite close to $28.1 \mu \mathrm{g}$ and $40 \mu \mathrm{g}$ lung burdens previously observed to induce inflammatory and fibrogenic effects in vivo $[40,78,105]$. Additional TEM images suggested that $0.0625 \mu \mathrm{g} / \mathrm{cm}^{2}$ ND-MWCNT and MWCNT-7 pierced or were taken up by WI-38 over $48 \mathrm{~h}$ (Figure 3.8). WI-38 directly exposed to $0.0625,0.125$, 0.250 , or $1.25 \mu \mathrm{g} / \mathrm{cm}^{2}$ ND-MWCNT or MWCNT-7 over a $48 \mathrm{~h}$ period showed that viability trended downward for all ND-MWCNT exposures beginning with $0.125 \mu \mathrm{g} / \mathrm{cm}^{2}$ and that exposure to $1.25 \mu \mathrm{g} / \mathrm{cm}^{2}$ ND-MWCNT significantly decreased WI-38 viability compared to control and $0.125 \mu \mathrm{g} / \mathrm{cm}^{2}(\mathrm{p}<0.0247, \mathrm{p}$ $<0.0197$ ) (Figure 3.9 C). Total live cell counts trended parallel to cell viability; additionally, total live cell numbers were significantly lower in WI-38 exposed to $1.25 \mu \mathrm{g} / \mathrm{cm}^{2}$ ND-MWCNT $(p<0.0235)$ (Figure 3.9 D). A one-way ANOVA and Tukey's post hoc test was used to compare between treatment groups.

\subsection{Recombinant IL-6 and IL-8 at biologically relevant concentrations significantly alter WI-38} proliferation.

CellTrace CFSE dye dilution assays were carried out to determine the impact of IL- 6 and IL-6 + IL-8 on WI38 proliferation. IL-6 has traditionally been linked to regulating cell proliferation and was therefore the focus of these experiments. Interestingly, IL-6 and the combination of IL- 6 and IL-8 were shown to significantly decrease proliferation of WI-38 after a $48 \mathrm{~h}$ exposure. MFI values used in Figure 3.8 are reported as the average of three independent FITC median values. Compared to control (MFI: $639 \pm$ 40.71), IL-6 “M7” (MFI: $760 \pm$ 19.97), IL-6 0.1 ng/mL (MFI: $839.7 \pm 11.92)$, IL-6 + IL-8 “ND” (MFI: $768.3 \pm$ 24.83), IL-6 + IL-8 “M7" (MFI: $792 \pm 19.6)$, and IL-6 + IL-8 $0.1+2.0$ ng/mL (MFI: $902.5 \pm 19.5$ ) exposed WI38 were believed to proliferate slower as suggested by significant increases in FITC median values which suggests fewer cells progressed beyond time 0 (Figure 3.10 A). Significant differences were also noted 
between IL-6 “ND” and IL-6 $0.1 \mathrm{ng}(\mathrm{p}<0.0028)$, IL-6 “ND” and IL-6 + IL-8 “M7” ( $p<0.0356)$, IL-6 “ND”, IL6 "M7", and IL-6 + IL-8 “ND" and IL-6 + IL-8 $0.1+2.0 \mathrm{ng}(\mathrm{p}<0.0004, \mathrm{p}<0.0222, \mathrm{p}<0.0332)$ (Table 3.2). These results suggest that biologically relevant concentrations of IL- 6 can impact WI-38 proliferation, and that the addition of IL-8 has no impact on proliferation when compared to IL-6 "M7" alone, further supporting that IL- 6 is the primary driver of the decrease in cell proliferation. Additionally, a time 0 sample was collected concurrent with WI-38 exposure to $0.0625,0.125,0.250$, or $1.25 \mu \mathrm{g} / \mathrm{cm}^{2}$ of NDMWCNT or MWCNT-7 to determine if cell proliferation changed during the duration of exposure of $48 \mathrm{~h}$. Data was collected from three biological replicates and averaged FITC median values were analyzed by one-way ANOVA and Tukey's post hoc test, with higher means suggesting that more cells did not divide. Results suggested that exposure to ND-MWCNT at 0.250 (MFI: $1205 \pm 26.01$ ) and $1.25 \mu \mathrm{g} / \mathrm{cm}^{2}$ (MFI: $1535 \pm 28.39$ ) significantly decreased WI-38 proliferation compared to control (MFI: $1075 \pm 72.42$ ), suggesting that the ND-MWCNT have greater impact on WI-38 activity $(p<0.0463, p<0.0001)$ (Figure 3.10 B). $1.25 \mu \mathrm{g} / \mathrm{cm}^{2}$ MWCNT-7 (MFI: $1484 \pm 13.30$ ) also significantly decreased WI-38 proliferation, but not lower exposure doses of MWCNT-7 ( $p<0.0001)$ (Figure 3.10 B). Additionally, no significance was noted between particles for the same exposure dose, but statistical significance was observed between all doses and particles compared to $1.25 \mu \mathrm{g} / \mathrm{cm}^{2}$ ND-MWCNT and MWCNT-7 (Table 3.3). These data suggest that the exposure dose plays a large role in limiting proliferation, possibly due to progressive loss of cell function at higher doses.

\subsection{Recombinant IL-6 and IL-8 at biologically relevant concentrations and direct exposure to ND- MWCNT significantly alter Collagen I production in WI-38}

To better understand how IL-6, IL-8, and the combination thereof may affect fibroblast activity, collagen was probed for using a total Collagen I antibody. WI-38 were exposed to IL-6, IL-8, or in combination or $0.0625,0.125,0.250$, or $1.25 \mu \mathrm{g} / \mathrm{cm}^{2}$ ND-MWCNT or MWCNT-7 over $48 \mathrm{~h}$, and whole cell lysates were collected using RIPA buffer with protease and phosphatase inhibitors. Lysates were subjected to SDS- 
PAGE, transferred to PVDF membranes, and exposed to Collagen I antibody and $\beta$-actin antibody. Collagen I expression increased at $48 \mathrm{~h}$ in cells exposed to "ND" and "M7" concentrations of IL-6, IL-8, and IL-6 + IL-8 (Figure 3.11 A). These results suggest that treatment with biologically relevant concentrations of IL- 6 and IL-8 can induce subtle alterations in WI-38 activity. Collagen I expression was increased at $48 \mathrm{~h}$ in cells exposed to $0.0625 \mu \mathrm{g} / \mathrm{cm}^{2}$ ND-MWCNT, while exposure to MWCNT-7 at all doses appeared to have no effect or decreased expression of collagen I (Figure 3.11 B). The highest exposures appeared to significantly decrease collagen I, but is most likely related to a decrease in cell function at such a high exposure. These results suggest that a very low exposure to CNT may be more pro-fibrogenic than high exposures. qRT-PCR was also utilized to assess col1A1 gene expression which was found to be significantly decreased in WI-38 exposed to $1.25 \mu \mathrm{g} / \mathrm{cm}^{2}$ ND-MWCNT and MWCNT-7 compared to control, further supporting the western blot results and decrease in cell function at high exposure doses (Figure 3.11 C). A one-way ANOVA and Dunnett's post hoc test was used for statistical analysis.

\subsection{0 $\alpha$-SMA gene expression is increased by low exposures to ND-MWCNT}

During fibrosis, fibroblasts may differentiate into more active myofibroblasts which have been linked to increased contractility, wound healing, and increased collagen production [155]. WI-38 were exposed to $0.0625,0.125,0.250$, or $1.25 \mu \mathrm{g} / \mathrm{cm}^{2}$ ND-MWCNT or MWCNT-7 over $48 \mathrm{~h}$. qRT-PCR was used to determine gene expression of $\alpha$-SMA, which was found to be significantly increased in WI-38 exposed to 0.0625 and $0.125 \mu \mathrm{g} / \mathrm{cm}^{2}$ ND-MWCNT, suggesting again that lower exposure doses induced a greater response in WI-38 $(p<0.0258, p<0.0005)$. A one-way ANOVA and Dunnett's post hoc test was used to compare between treatments (Figure 3.12).

\section{Discussion:}

A wide variety of functionalized MWCNT are now available, each with a unique set of physicochemical characteristics that allow for a multitude of uses in industrial and consumer products, 
but may also imbue them with equally unique toxicities within the human lung. Streamlined, efficient, and realistic assessment of these nanofibers for potential toxicity has been hampered by a lack of standardized in vitro methods, exposure doses, and understanding of how physicochemical properties can impact CNT interactions with cells and tissues that generate toxicity. This study focused on understanding the in vitro pro-fibrogenic potential of ND-MWCNT and MWCNT-7 through the use of two in vitro methods that addressed specific epithelial-fibroblast communication and direct exposure of fibroblasts to ND-MWCNT and MWCNT-7 in order to begin addressing these issues.

The role of epithelial-fibroblast communication plays an important part in the development and maintenance of pulmonary fibrosis. It is hypothesized that fibrosis may be the result of alveolar epithelial injury that induces resident fibroblasts to migrate, proliferate, and become active myofibroblasts which deposit ECM and can act through paracrine signals to maintain alveolar epithelium damage [154]. We chose to focus on the role of epithelial-driven signaling on WI-38 fibroblasts, electing to focus on IL-6 and IL-8, which have been shown to promote pulmonary fibrotic outcomes in vitro and in human patients [156-159]. Additionally, although foreign material is typically excluded from the interstitial space by the protective epithelial layer, the needle-like qualities of MWCNT as well as their surface characteristics promote their passage into and beyond alveolar epithelial cells where they could directly interact with fibroblasts $[78,79,105]$. For this reason, we also chose to independently assess the impact of direct exposure to ND-MWCNT and MWCNT-7 on WI-38 to determine if their physicochemical differences would alter their bioactivity (Figure 3.5).

While inhaled foreign material is typically whisked away by macrophage and the mucociliary escalator within the lung, the aerodynamic diameter of the material determines where it will settle, with particles below $5 \mu \mathrm{m}$ typically depositing in the bronchioles and alveoli $[160,161]$. For MWCNT, the MMAD can significantly impact the alveolar deposition rate inversely; the higher the MMAD, the lower the deposition [57]. These considerations are important to the design of an in vivo study, and 
consequently, to in vitro studies. Based upon values utilized by Porter et al. in 2010 [40], assuming 400 $\mu \mathrm{g} / \mathrm{m}^{3} \mathrm{MWCNT}$ aerosol concentration, MMAD of $1.5 \mu \mathrm{m}$ for MWCNT-7, minute ventilation of $20 \mathrm{~L} / \mathrm{min}$, and alveolar deposition of 30 percent, the in vitro exposure doses to BEAS-2B of 1.25 and $2.50 \mu \mathrm{g} / \mathrm{cm}^{2}$ would roughly correlate with a human working 4.6 to 9.2 years, respectively, under such conditions. Similarly, if one used the same calculation for the WI-38 exposure dose range from 0.0625 to 1.25 $\mu \mathrm{g} / \mathrm{cm}^{2}$, the correlation to a human exposure would range from about 2.8 months to 4.6 years under the above working conditions described. It is important to note that these values are extrapolations based upon specific conditions in a lab prior to installation of engineering controls [46]; a more recent publication suggested that workers in the CNT industry are exposed to an average of $10.6 \mu \mathrm{g} / \mathrm{m}^{3}$ [57]. Recalculating the human extrapolation doses based upon this airborne concentration and $11 \%$ alveolar deposition, the same dose range $\left(0.0625\right.$ to $\left.1.25 \mu \mathrm{g} / \mathrm{cm}^{2}\right)$ is reflective of 23.7 to nearly 475 years, highlighting the difficulty of determining the relevancy of an exposure dose as the field moves forward and workplaces begin adhering to the Recommended Exposure Limit (REL) of $1.0 \mathrm{\mu g} / \mathrm{m}^{3}$ [106]. Information on the MMAD and alveolar deposition rate was unavailable for the specific ND-MWCNT used in this study; however, it was suggested that the MMAD of ND-MWCNT and MWCNT-7 were similar (personal communication).

As assessed by TEM, ND-MWCNT and MWCNT-7 were observed piercing BEAS-2B human bronchial epithelial cells and in agglomerates within vesicles at 1.25 and $2.50 \mu \mathrm{g} / \mathrm{cm}^{2}$ (Figure 3.1, 3.2). Similarly, ND-MWCNT and MWCNT-7 were observed in agglomerates and piercing WI-38 fibroblasts over a dose range from 0.0625 to $2.50 \mu \mathrm{g} / \mathrm{cm}^{2}$ (Figures 3.6, 3.8). Although Briand et al. suggested that pristine and amino-functionalized MWCNT were capable of similar uptake in various epithelial and fibroblast cell lines regardless of surface charge or functionalization [162], others have shown that CNT hydrophilicity can significantly enhance cytotoxicity in vitro due to increased interaction with and uptake into cells compared to hydrophobic MWCNT [163]. Personal observation and those made by colleagues 
suggest that the ND-MWCNT used in this study are slightly more hydrophilic that MWCNT-7 by the comparative ease with which they are suspended, thus providing one potential reason for why NDMWCNT appeared more bioactive in assays assessing cytotoxicity (Figure 3.3 A, Figure 3.7, Figure 3.9 C, D). Although it has been published that one can more specifically determine the hydrophobicity of MWCNT using $n$-octanol/water partitioning [164], OECD guidelines suggest that typical protocols to determine Kow values are inappropriate and unreliable since MWCNT have difficulty crossing the octanol-water boundary $[165,166]$, which was also personally verified (unpublished data). Additionally, hydrophilicity is typically correlated with increased dispersibility and less formation of large agglomerates or bundled CNT; since agglomerated MWCNT are known to be less toxic in vitro and in vivo, it is possible that the ND-MWCNT generally were better dispersed and reached higher concentrations of free single fibers thereby inducing increased bioactivity in WI-38. Although not a primary focus of this study, particle settling and agglomeration may also have been altered in solution over the $48 \mathrm{~h}$ exposure depending upon the total number of ND-MWCNT or MWCNT-7 delivered, especially at the highest doses in which increased MWCNT-7 hydrophobicity could increase subsequent formation of agglomerates with lower surface area-to-mass ratios (and consequently, less reactive surface area) [7].

A pro-inflammatory multiplex showed significant increases in IL-13, IL-6, and IL-8 in conditioned media from BEAS-2B exposed to ND-MWCNT or MWCNT-7 for $24 \mathrm{~h}$. Although IL-13 has been implicated in pulmonary fibrosis, the concentration of this cytokine was prohibitively low to justify further testing at biologically relevant concentrations with available recombinant IL-13 (Figure 3.4 A). Instead, we chose to further study the effects of IL- 6 and IL-8 on WI-38 using biologically relevant concentrations derived directly from the multiplex results for ND-MWCNT and MWCNT-7 exposure at $2.50 \mu \mathrm{g} / \mathrm{cm}^{2}$ in BEAS-2B as well as the manufacturer's suggested dose. Results suggested dose and particle-dependent increases in IL-6 and IL-8 in BEAS-2B, with only the $2.50 \mu \mathrm{g} / \mathrm{cm}^{2}$ exposure dose inducing significant 
production of these cytokines compared to DM only (Figure 3.4 B, C). Together, IL-6 and IL-8 have been implicated in the pulmonary inflammatory and fibrotic response [156, 157, 167-169], and also shown to play an important role in the biological response to MWCNT [76, 95, 142, 170-172]. Dong et al. noted significant increases in IL-6 gene expression and production in mouse lung tissue and BAL fluid shortly after an occupationally relevant exposure to MWCNT-7. More critically, a recently published epidemiological study comparing a control group to workers exposed to MWCNT on the job for at least one year showed a 5-fold increase in IL-6 and 2-fold increase in IL-8 in sputum samples, suggesting that these cytokines may participate in the development of pulmonary disease in workers chronically exposed to MWCNT [170].

WI-38 exposure to IL- 6 and IL-8 only over $48 \mathrm{~h}$ indicated that in the absence of other cell signaling molecules such as TGF $\beta$ or PDGF typically associated with MWCNT exposure and pulmonary fibrosis, WI-38 did not generate a strong fibrogenic response. These data suggest that IL-6 and IL-8 may play a supporting, but not primary, role in MWCNT-induced fibrosis. IL-6 and IL-8, regardless of exposure concentration, did not alter WI-38 viability or total cell counts (Figure 3.9 A, B). However, exposure to IL- 6 alone at higher concentrations or IL-6 and IL-8 together significantly decreased WI-38 proliferation (Figure 3.10 A). While fibroblast proliferation is usually considered a hallmark of the disease state, the role of IL-6 in fibroblast proliferation has been contested since the early 1990s. Early studies on the role of IL- 6 in pulmonary fibrotic diseases suggested that IL- 6 inhibited fibroblast proliferation. Yoshida et al. showed that overexpression of IL-6 in rat lungs induced an inflammatory response with limited changes in fibroblast proliferation, while TGF $\beta$ and PDGF induced a fibrotic phenotype with enhanced fibroblast proliferation, suggesting that perhaps IL-6 does not have a direct role in fibrogenesis [173]. Alveolar fibroblasts collected from patients with sarcoidosis produced high levels of IL-6, which through an autocrine manner, inhibited fibroblast proliferation and therefore limited the extent of the fibrotic response that could occur in these patients [174]. Other studies have 
shown that attenuating IL-6 signaling limits pulmonary fibrosis $[156,168]$. However, it is more likely that IL-6 has pleiotropic effects, and can suppress or increase proliferation dependent upon the cell line studied and presence of other cell signaling molecules. For instance, Rose-John observed that IL-6 inhibited growth of NIH/3T3 fibroblasts [175], while others observed IL-6 upregulation was critical to fibroblast activation and proliferation $[176,177]$. In this context, the role of IL-6 is still undetermined and will require further study by others to fully elucidate its effects and correlation between in vitro and in vivo studies.

WI-38 were also exposed to ND-MWCNT and MWCNT-7 directly over $48 \mathrm{~h}$ and assessed for proliferation. Our results showed an inverse relationship between exposure dose and proliferation, such that the highest dose $\left(1.25 \mu \mathrm{g} / \mathrm{cm}^{2}\right)$ of each CNT decreased proliferation, as well as the 0.250 $\mu \mathrm{g} / \mathrm{cm}^{2}$ ND-MWCNT dose. These data suggest that the higher doses utilized in this study (which are still remarkably low compared to a majority of in vitro studies on MWCNT) significantly impact the proliferative ability of WI-38, while low doses appeared to have no effect (Figure 3.10 B). Although we expected to see enhanced WI-38 proliferation after direct exposure to show a "hallmark of fibrosis" in vitro, we determined that this endpoint, while relatively straightforward to study, is perhaps not best suited for the in vitro model systems utilized here. The complex molecular events that occur over a period of weeks in vivo is difficult to recapitulate over a $48 \mathrm{~h}$ time period using normal lung fibroblasts such as WI-38. However, Mishra et al. studied MWCNT-7 induced proliferation using CRL-1490 fibroblasts and noted that the lowest doses between 0.002 and $0.06 \mu \mathrm{g} / \mathrm{cm}^{2}$ increased proliferation, while exposure to 0.6 and $2.0 \mathrm{\mu g} / \mathrm{cm}^{2}$ significantly decreased proliferation, which generally matches our results \{Mishra, $2012 \# 297\}$. It is important to note that WI-38 and CRL-1490 were originally derived from very different donors (fetus and 7 y.o. child), have different DNA profiles, and may also have different population doubling times. It is also of interest to note that an exposure of $0.002 \mu \mathrm{g} / \mathrm{cm}^{2}$ would be the equivalent of exposing a mouse to $1 \mu \mathrm{g}$ MWCNT, which has not been observed to cause 
fibrosis in vivo; however, this in vitro data suggests that perhaps the lowest concentrations possible, even with limited correlation with negative effects in vivo, may be necessary for nanotoxicology studies [110]. It should also be noted, nonetheless, that others have shown increased fibroblast proliferation at up to nearly 20-fold higher exposure doses compared to our study in other fibroblast cell lines, further supporting that the choice of cell line and assay utilized is essential to achieving desirable results, but may not necessarily be reflective of a feasible real-life exposure especially delivered as a short-term, one-time exposure dose in vitro [150].

Collagen I is important because it is one of the primary extracellular matrix proteins deposited by activated fibroblasts and contributes to the fibrotic phenotype. While IL- 6 and IL-8 appeared to have a more subtle effect upon collagen I production (Figure 3.11 A), $0.0625 \mu \mathrm{g} / \mathrm{cm}^{2}$ ND-MWCNT consistently induced protein expression higher than that of MWCNT-7 at the same exposure dose as well as compared to higher doses of either CNT (Figure 3.11 B). qRT-PCR to assess changes in col1A1 gene expression roughly matched protein expression, and also supported our conclusion that WI-38 exposed to the highest doses of ND-MWCNT or MWCNT-7 possibly begin to lose their typical function (Figure 3.11 C). Gene expression of $\alpha$-SMA was also assessed in WI-38 directly exposed to CNT, and results suggested that the lowest doses $\left(0.0625\right.$ and $\left.0.125 \mu \mathrm{g} / \mathrm{cm}^{2}\right)$ of ND-MWCNT induced higher expression of this protein typically associated with myofibroblast activation (Figure 3.12). Myofibroblasts are often found in the fibroblastic foci within IPF lungs, and are predominately responsible for excessive extracellular matrix deposition [154]. It is believed that TGF $\beta$ is a primary driver of this process and maintenance of the phenotype, but was not significantly increased in our in vitro system (data not shown), suggesting that perhaps the ND-MWCNT were inducing $\alpha$-SMA gene expression through an alternative mechanism. Others have shown that MWCNT exposure induced $\alpha$-SMA production as well in NIH 3 T3 murine fibroblasts at 30 or $60 \mu \mathrm{g} / \mathrm{mL}$ of MWCNT and WI-38 VA-13 (which are SV-40 
transformed with an unstable karyotype) at $20 \mu \mathrm{g} / \mathrm{mL}$, which are significantly higher doses than what we used in our study.

Together, the results suggest that direct treatment with ND-MWCNT or MWCNT-7 induced increased bioactivity in WI-38 suggestive of a potential fibrotic phenotype as opposed to exposure to biologically relevant concentrations of epithelial-derived IL-6 or IL-8. Additionally, ND-MWCNT were observed to induce greater responses in WI-38 which is not in concordance with an unpublished in vivo study suggesting that the ND-MWCNT were less fibrogenic than MWCNT-7 at occupationally relevant doses. We find this to be an important point of discussion, especially with increased desire for reliance on in vitro studies to make regulatory decisions. Several possible reasons exist for the discordance between in vitro and in vivo results, which will be discussed below:

1. Effects of dispersion and hydrophobicity: It has been well established that agglomerated MWCNT are less capable of inducing toxic effects in vitro and in vivo, and that surface chemistry influences the degree of agglomeration. It was assumed that MWCNT-7 were more hydrophobic than ND-MWCNT, and therefore, within an aqueous environment, more prone to agglomerating over the duration of exposure. It is likely that the more hydrophilic ND-MWCNT were present as single fibers instead of large agglomerates, suggesting that the WI-38 despite receiving the same exposure dose of ND-MWCNT and MWCNT-7 potentially received a more potent delivered dose of ND-MWCNT.

2. Protein corona: MWCNT are known to associate with proteins found within the lung and in cell culture media which may impart changes on their uptake and biodistribution. The composition of the protein corona, composed of proteins adsorbed on the particles' surface, is strongly dependent upon the surface, size, and concentration of particles studied, as well as the total protein concentration in the bodily fluid or cell media the particles are within [178]. It has been observed that the numerous defect sites usually present in ND-MWCNT 
allow for quite more extensive protein adsorption compared to pristine MWCNT with limited defects [179], which suggests that differences in the quantity and type of proteins on the surface could alter their bioactivity and change their "biologically active identity" (how cells "see" a nanomaterial). Work by Maiorano et al. also showed that cell media composition (comparing DMEM to RPMI) significantly altered protein corona formation on the same particles and subsequent bioactivity, which is important to further understanding the wide breadth of divergent in vitro results in nanotoxicology [178]. It also may be possible that corona formation could be quite different in vitro and in vivo, hence providing another possible reason for why ND-MWCNT in vivo/in vitro results are not in complete concordance compared to pristine MWCNT-7.

3. Presence of surface defects: In addition to potentially changing protein adsorption, surface defects also provide increased reactive surface area. Since the ND-MWCNT are known to have more defective sites, it is possible that these sites provide increased surface area to interact with cells and enhance their uptake and subsequent bioactivity. Within the monoculture WI-38 system used here, it is within reason to assume that increased cellular interaction and uptake could also increase bioactivity. However, this system could not account for interactions with other cell types such as macrophage prior to reaching fibroblasts directly, which could have a drastic impact on the total number of particles that would reach and activate the fibroblasts in vivo. Additionally, ND-MWCNT with surface defects were shown to completely degrade in the presence of biologically-relevant concentrations of $\mathrm{HRP} / \mathrm{H}_{2} \mathrm{O}_{2}$ compared to pristine or carboxylated MWCNT, thus suggesting that over the course of an in vivo study, the ND-MWCNT would likely also break down much faster than MWCNT-7, which clearly could not be a consideration over $48 \mathrm{~h}$ in vitro [153]. 
4. Choice of cell line: The WI-38 used in this study are considered "normal" human lung fibroblasts and were treated with a one-time exposure over $48 \mathrm{~h}$. In vivo, fibrosis is not typically observed until at least 14 to 28 days (or longer) post-exposure to MWCNT, suggesting that an enormous number of changes must occur involving numerous cell types to induce the observed fibrotic phenotype. While it is understandable to want to observe nanomaterial induced toxicity on cells which are considered normal, it would perhaps also be useful to study their effects in cells representative of a disease state as well, such as by using IPF fibroblasts. While we could not discern de novo fibrotic signaling events induced by MWCNT in these cells, they would perhaps be a more effective model for comparing the fibrogenic potential of a panel of functionalized and pristine MWCNT under a compressed time frame without chronic exposure, and thus be more reflective of the in vivo state under which fibrosis is observed. 

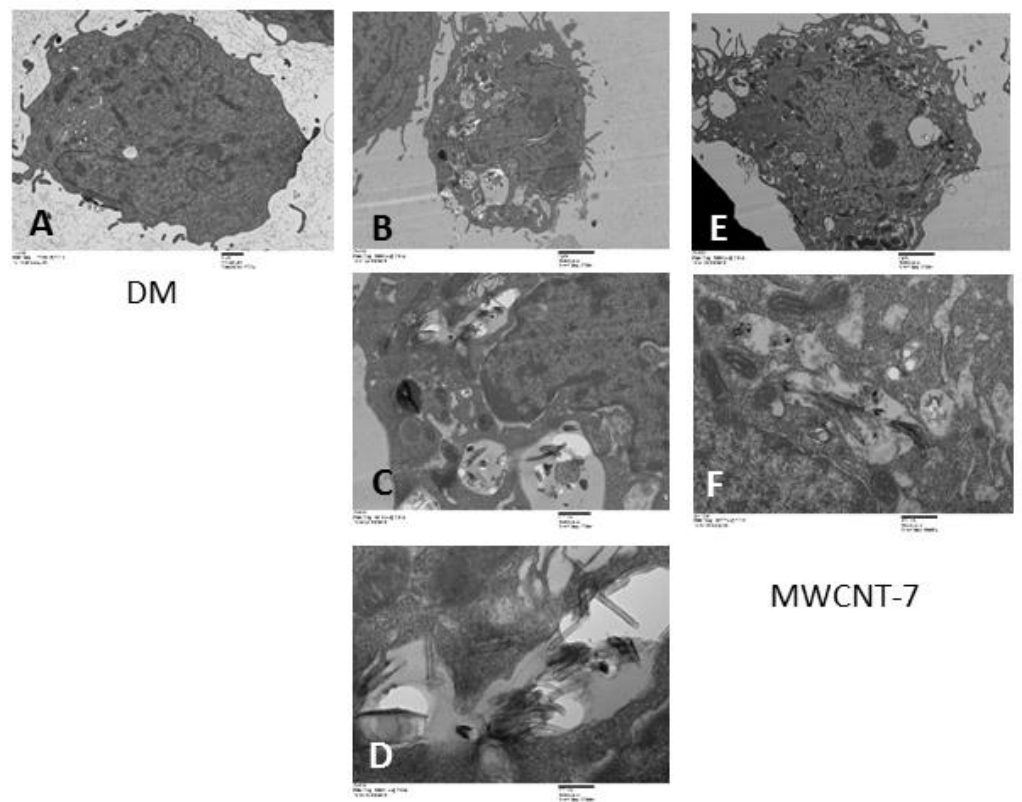

MWCNT-7

ND-MWCNT

$1.25 \mu \mathrm{g} / \mathrm{cm}^{2}$

Figure 3.1 BEAS-2B cells engulf ND-MWCNT and MWCNT-7. TEM analysis of BEAS-2B exposed to 1.25

$\mu \mathrm{g} / \mathrm{cm}^{2}$ of either ND-MWCNT (B, C, D) or MWCNT-7 (E, F) compared to dispersion media control over 24 hours. Images in $\mathrm{C}$ and $\mathrm{D}$, and $\mathrm{F}$ feature enhanced magnification to show detail from $\mathrm{B}$ and $\mathrm{E}$, respectively. 


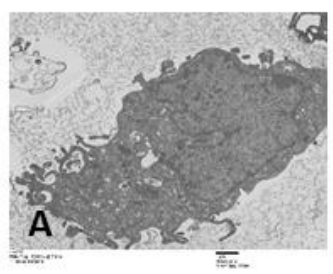

DM
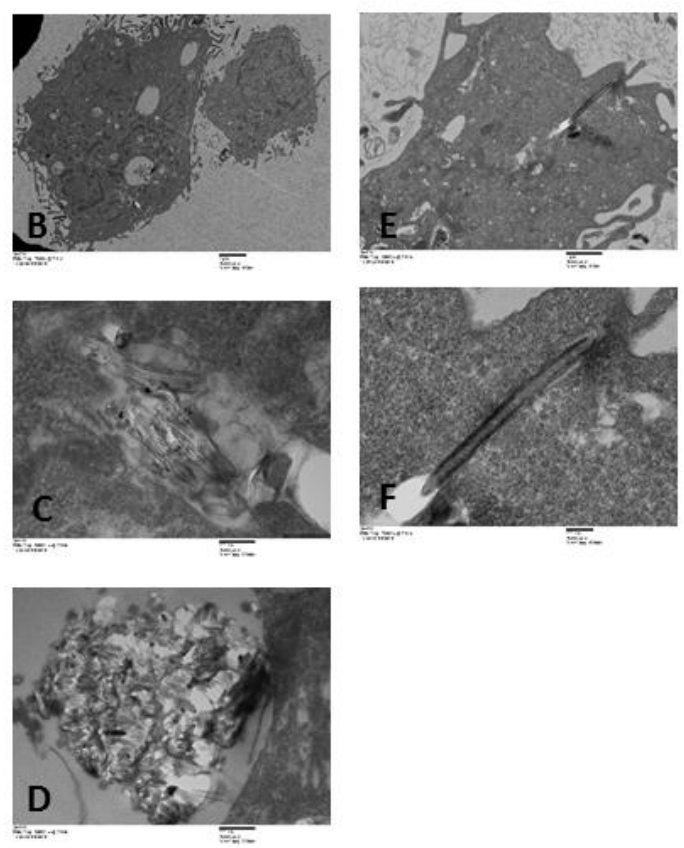

ND-MWCNT
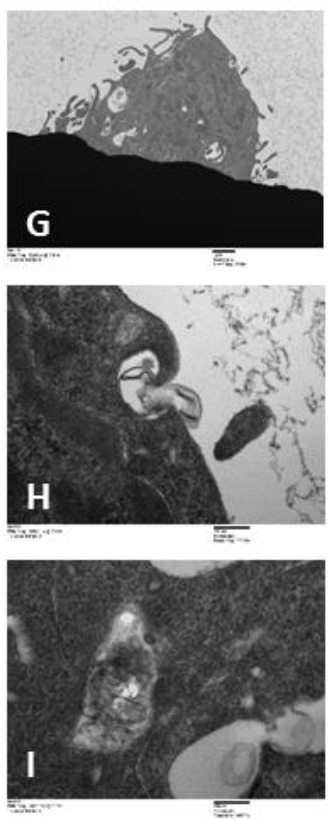

MWCNT-7

\section{$2.50 \mu \mathrm{g} / \mathrm{cm}^{2}$}

Figure 3.2 BEAS-2B cells engulf ND-MWCNT and MWCNT-7. TEM analysis of BEAS-2B exposed to 2.50 $\mu \mathrm{g} / \mathrm{cm}^{2}$ of either ND-MWCNT (B, C, D, E, F) or MWCNT-7 (G, H, I) compared to dispersion media control over 24 hours. Images in $\mathrm{C}$ and $\mathrm{D}, \mathrm{F}$, and $\mathrm{H}$ and I feature enhanced magnification to show detail from $\mathrm{B}$, $\mathrm{E}$, and $\mathrm{G}$ respectively. 


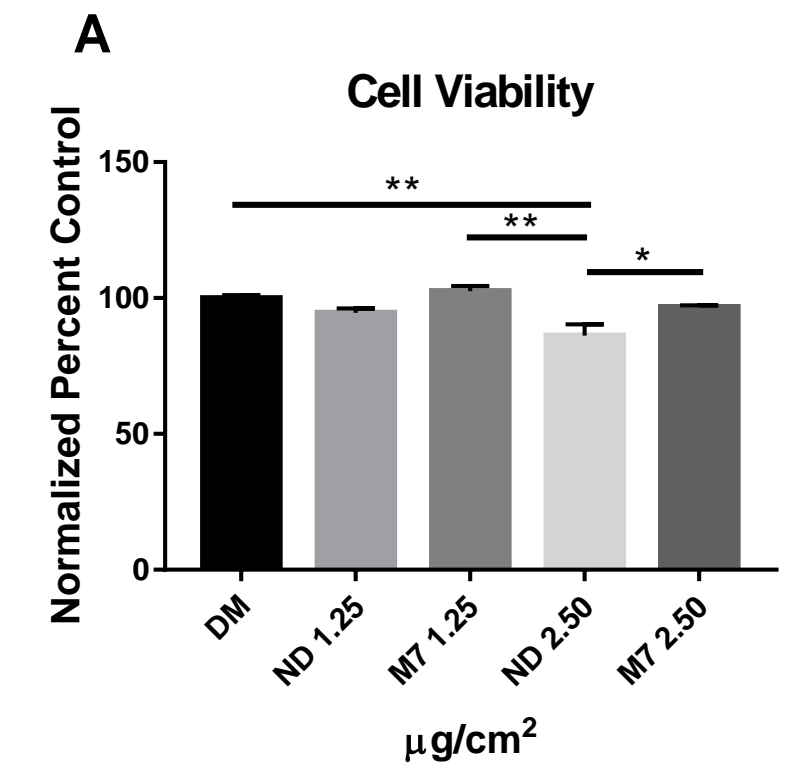

B

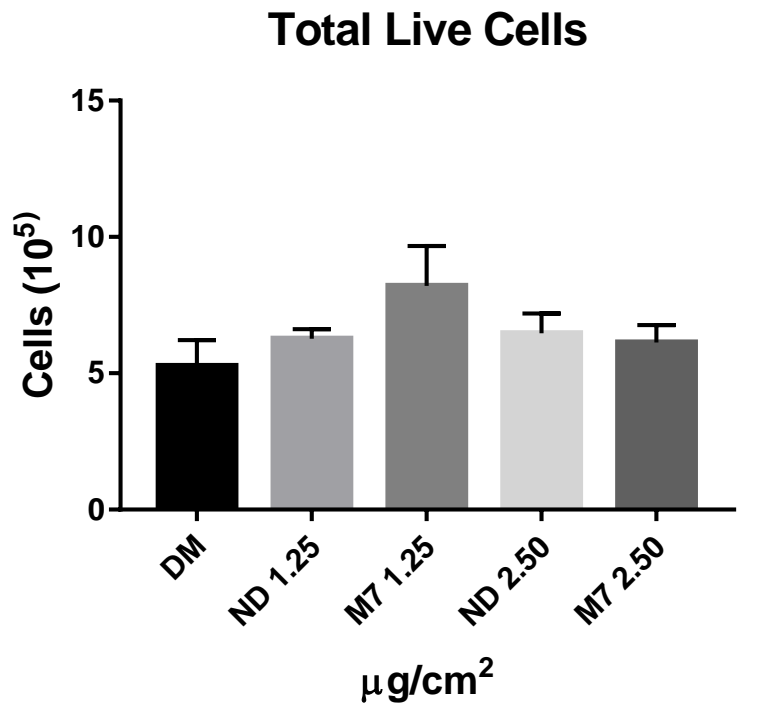

Figure 3.3 Effect of ND-MWCNT and MWCNT-7 on BEAS-2B viability and proliferation. Trypan Blue staining and a Countess Automated Cell Counter were used to assess BEAS-2B viability and proliferation over a $24 \mathrm{~h}$ period. Three independent biological samples were collected and results are presented here. A. Bars represent percent viability normalized to control and are presented as means \pm SEM $(*, p$ $<0.05^{* *}, \mathrm{p}<0.01$ ) compared between all groups. B. Bars represent total number of live cells counted and suggest no significant change in proliferation. 
A

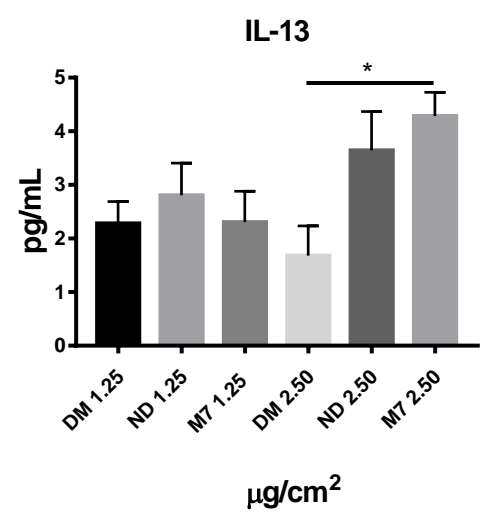

B

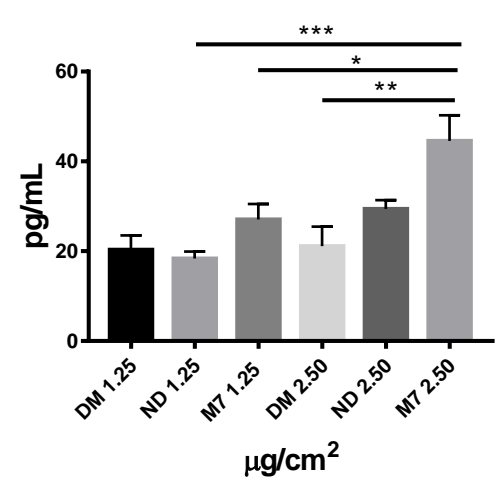

C

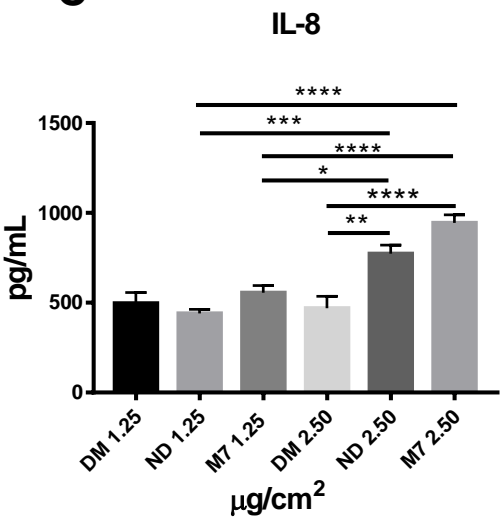

Figure 3.4. ND-MWCNT and MWCNT-7 induce pro-inflammatory cytokines in BEAS-2B in a dosedependent manner. BEAS-2B were seeded in 24 well plates in three independent experiments and treated with DM, ND-MWCNT, or MWCNT-7 over a $24 \mathrm{~h}$ period. Conditioned medium samples were collected and centrifuged to pellet CNT and applied to a Meso Scale Discovery V-PLEX Pro-inflammatory Panel 1 Kit. Significant increases were observed in A. IL-13, B. IL-6, and C. IL-8 production compared between controls and between exposure doses. Bars represent $\mathrm{pg} / \mathrm{mL}$ mean values of each cytokine \pm SEM. 
A 1.25 or $2.50 \mu \mathrm{g} / \mathrm{cm}^{2}$ NDMWCNT or MWCNT-7

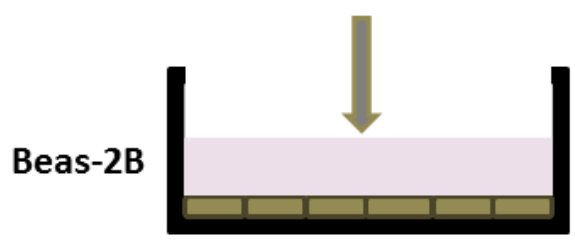

B

Recombinant IL-6

WI-38

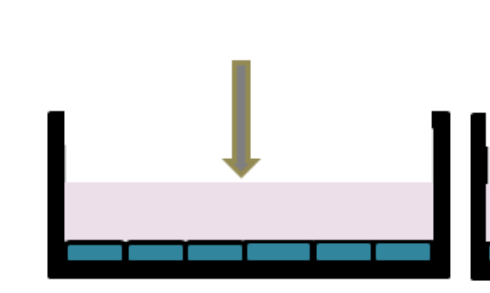

C

DM

Recombinant IL-8

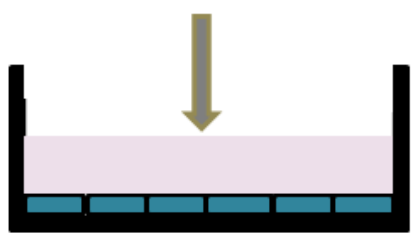

WI-38
ND-MWCNT

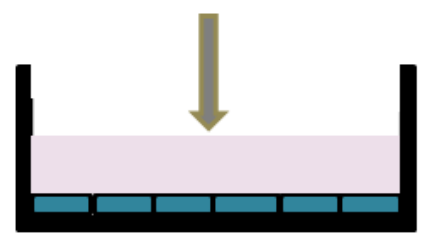

חים

Recombinant

IL-6 + IL-8

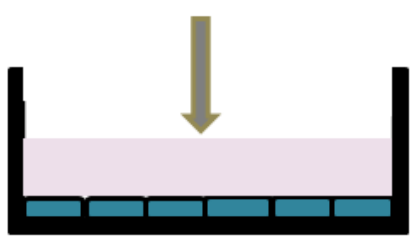

MWCNT-7

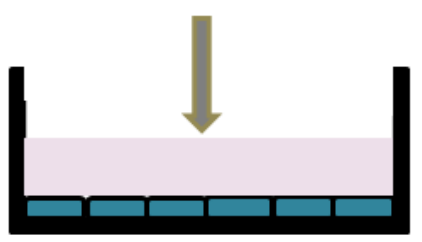

Figure 3.5 Experimental design to assess effects of biologically-relevant concentrations of IL-6 and IL-8 and direct exposure to ND-MWCNT or MWCNT-7 on WI-38 fibroblasts. A. Beas-2B were treated with 1.25 or $2.50 \mu \mathrm{g} / \mathrm{cm}^{2}$ and assessed for cytokine production. B. Concentrations of IL-6 and IL-8 present after ND-MWCNT and MWCNT-7 exposure at the $2.50 \mu \mathrm{g} / \mathrm{cm}^{2}$ dose were converted into $\mathrm{ng} / \mathrm{mL}$ concentrations and purchased recombinant IL-6, IL-8, or a combination of both were added to WI-38 for $48 \mathrm{~h}$ and downstream effects were monitored. C. In order to assess the effect of direct exposure to NDMWCNT or MWCNT-7 on WI-38, cells were exposed to DM, ND-MWCNT, or MWCNT-7 over $48 \mathrm{~h}$ prior to further study. 

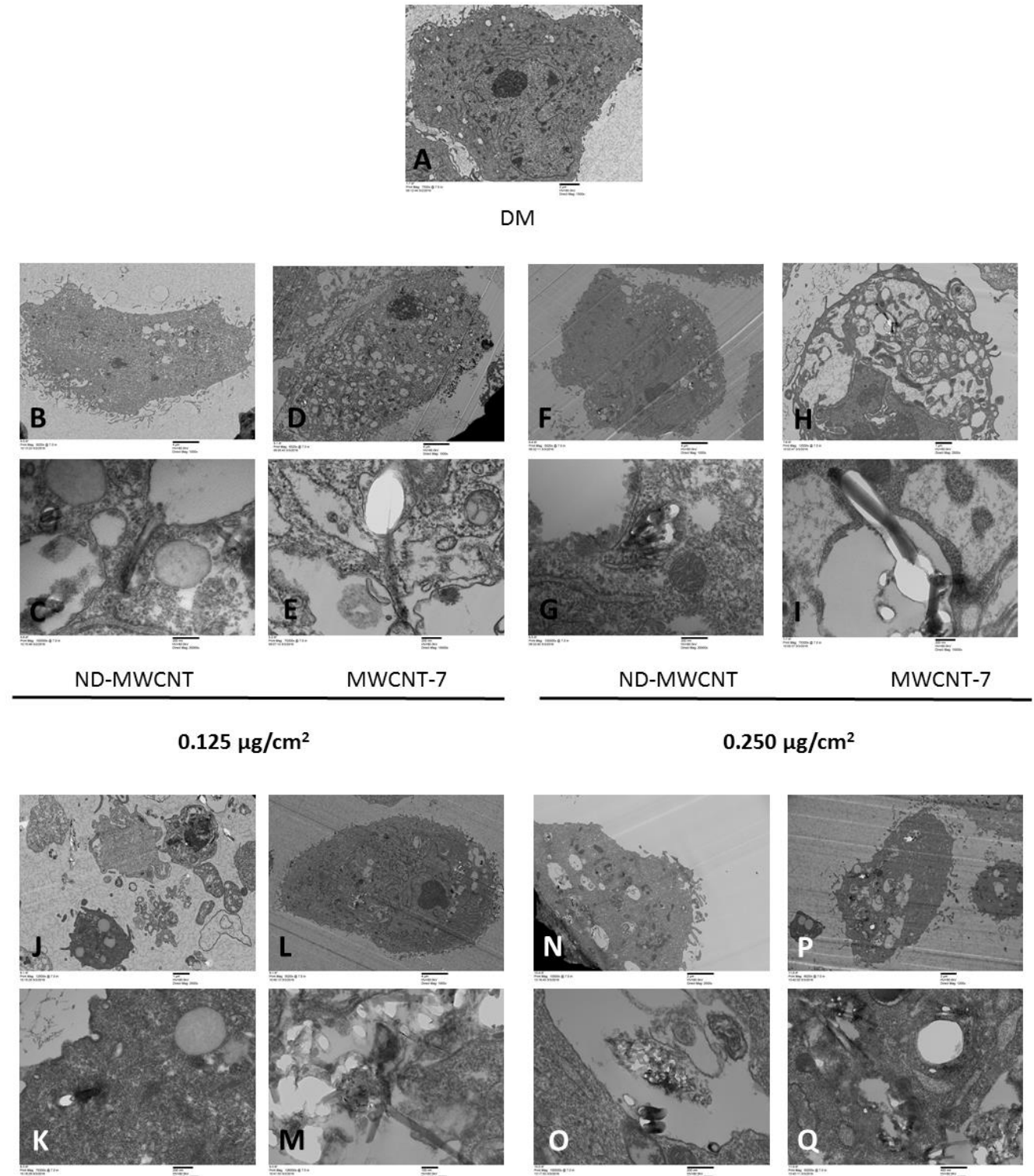

ND-MWCNT

MWCNT-7
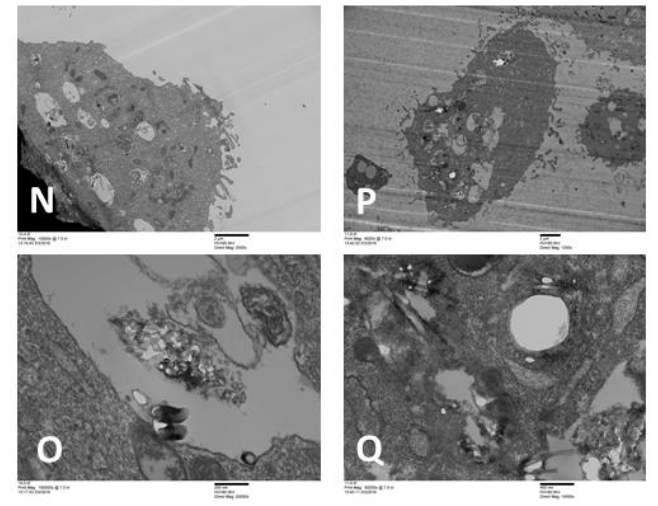

ND-MWCNT

MWCNT-7

$1.25 \mu \mathrm{g} / \mathrm{cm}^{2}$

$2.50 \mu \mathrm{g} / \mathrm{cm}^{2}$

Figure 3.6 WI-38 cells engulf or are pierced by ND-MWCNT and MWCNT-7. TEM analysis of WI-38 across a range of doses over 48 hours. 


\section{Cytotoxicity}

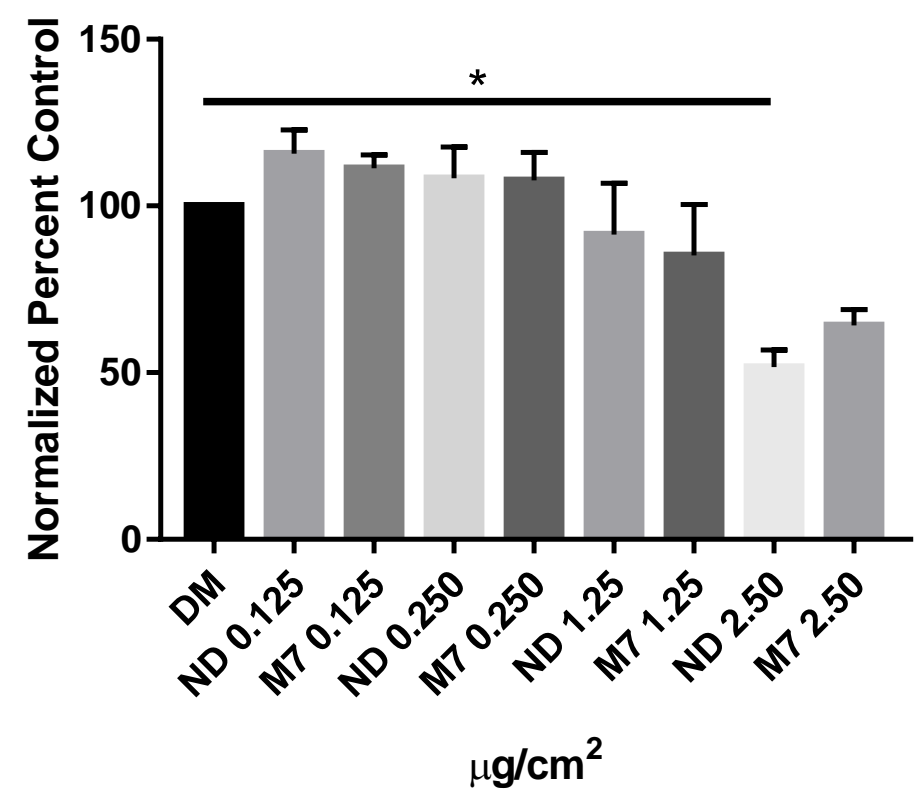

Figure 3.7 Direct exposure of WI-38 to ND-MWCNT and MWCNT-7 alters cytotoxicity assessed by MTS assay. WI-38 were exposed to DM, ND-MWCNT, or MWCNT-7 for 48 hours and assessed by CellTiter 96 Aqueous One (MTS) assay using a BioTek plate reader at 490nm. Results shown are from three independent experiments. A. Results suggest that the highest exposure of ND-MWCNT significantly induces cytotoxicity $(*, p<0.05)$. 

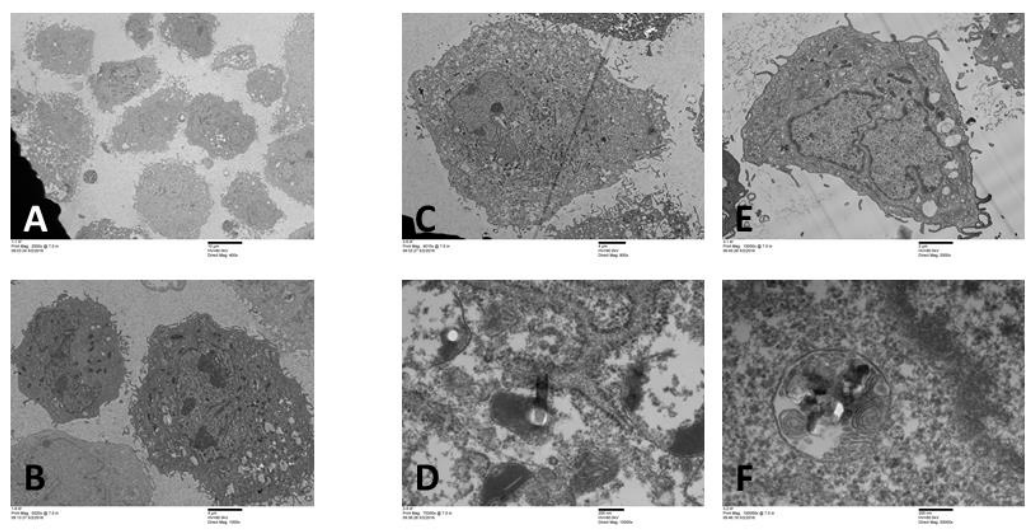

DM

ND-MWCNT

MWCNT-7

$0.0625 \mu \mathrm{g} / \mathrm{cm}^{2}$

Figure 3.8 ND-MWCNT and MWCNT-7 interact with WI-38 at lower exposure dose. TEM analysis of WI38 exposed to $0.0625 \mu \mathrm{g} / \mathrm{cm}^{2}$ to verify cellular uptake. 

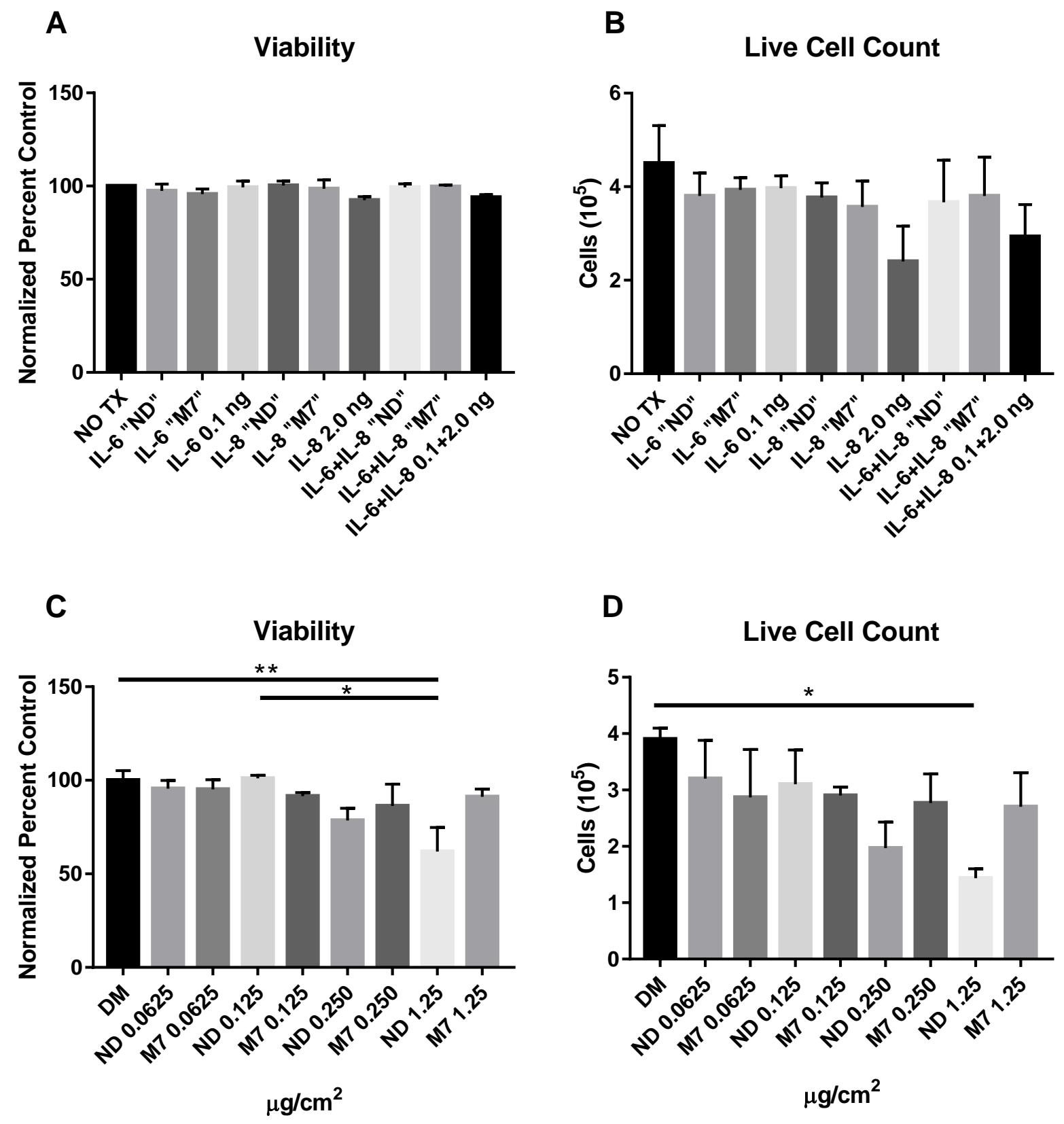

Figure 3.9 IL-6 and IL-8 induce limited effects on WI-38 viability and total cell counts but direct exposure reduces viability at high exposure doses. WI-38 were treated with biologically relevant concentrations of IL-6, IL-8, or a combination of IL- 6 + IL-8 as well as manufacturer's suggested concentrations. A. Bars represent percent viability normalized to control across three independent experiments and presented as means $\pm \mathrm{SEM}$; no significant difference was noted over $48 \mathrm{~h}$. B. Bars represent total number of live cells averaged across three independent experiments as means $\pm S E M$; 
similarly, no significant difference was noted. C. WI-38 were exposed to DM, ND-MWCNT, or MWCNT-7 across a range of doses over $48 \mathrm{~h}$. Bars represent percent viability normalized to control across three independent experiments and presented as means \pm SEM. A decrease in cell viability was observed at $1.25 \mu \mathrm{g} / \mathrm{cm}^{2}$ ND-MWCNT. D. Similarly, a decrease in total live cells at the same dose was observed. Bars

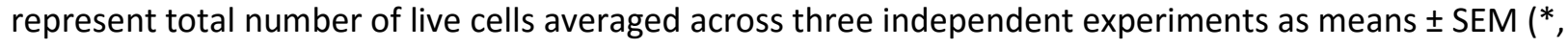
$\mathrm{p}<0.05, * *, \mathrm{p}<0.01)$. 
A

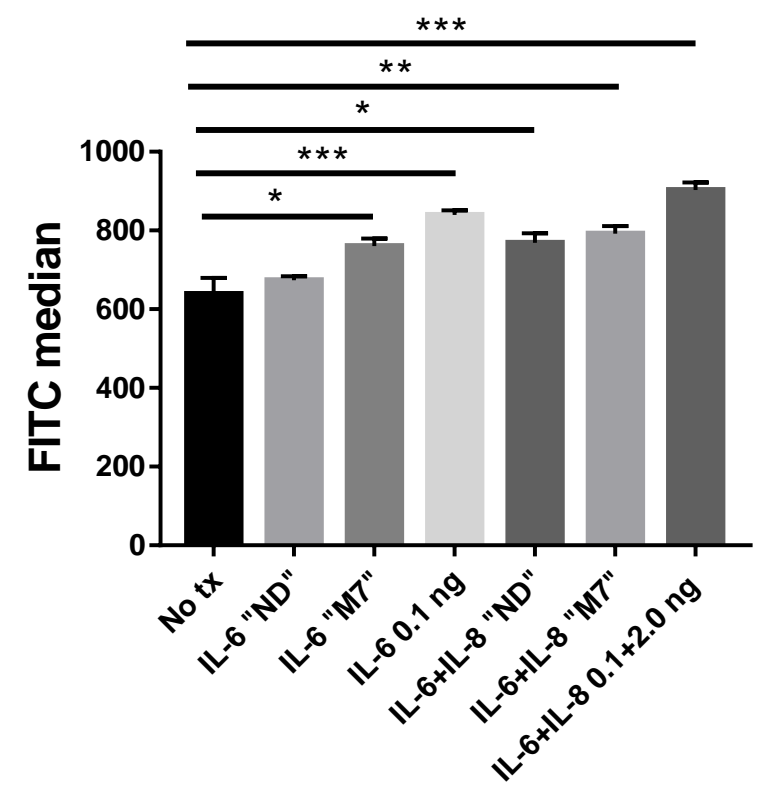

B

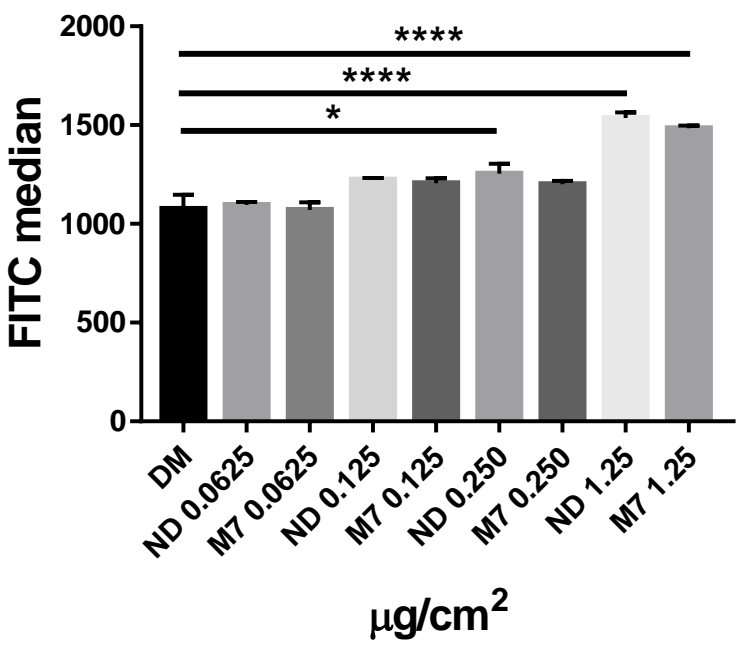

Figure 3.10 Recombinant IL-6 and IL-8 and direct exposure to ND-MWCNT and MWCNT-7 significantly alter WI-38 proliferation assessed by CellTrace CFSE dye. WI-38 were treated with various concentrations of IL-6, IL-8, or IL6 + IL-8 over a $48 \mathrm{~h}$ time period. Results are from a representative experiment in biological triplicate. A. Bars represent averages of FITC median MFI values \pm SEM. Compared to a control receiving no treatment with IL-6 or IL-8, results suggest that all treatments (aside from IL-6 "ND") induce a significant reduction in WI-38 proliferation. Table 3.8. shows additional significant relationships not shown on the bar graph. WI-38 were also exposed to DM, ND-MWCNT, or MWCNT-7 over a 48 hour time period. Results are from a representative experiment in biological triplicate. B. Bars represent averages of FITC median MFI values \pm SEM. Table 3.9. shows other significant relationships not shown on the bar graph. Compared to DM control, results suggest that WI38 exposure to 0.250 or $1.25 \mu \mathrm{g} / \mathrm{cm}^{2}$ ND-MWCNT or $1.25 \mu \mathrm{g} / \mathrm{cm}^{2}$ MWCNT-7 induce a significant reduction in WI-38 proliferation $\left(*, p<0.05,{ }^{* *}, \mathrm{p}<0.01,{ }^{* * *}, \mathrm{p}<0.001, * * * *, \mathrm{p}<0.0001\right)$. 
A
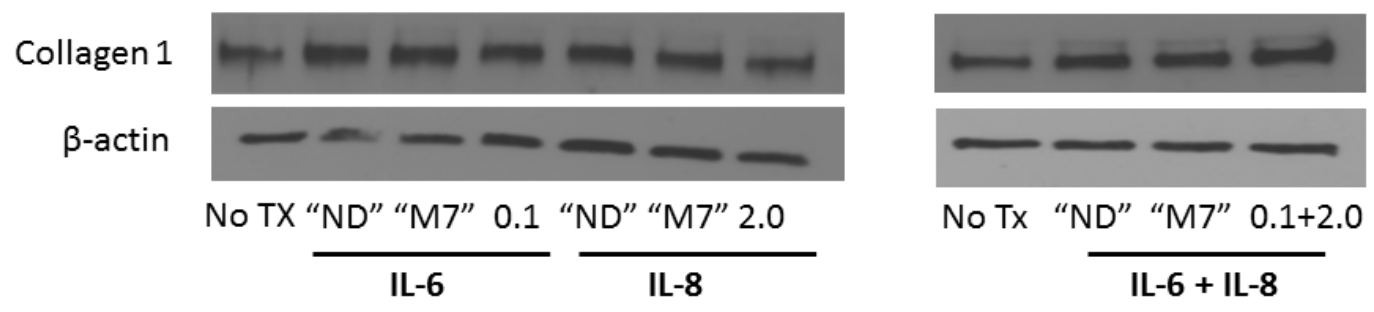

B
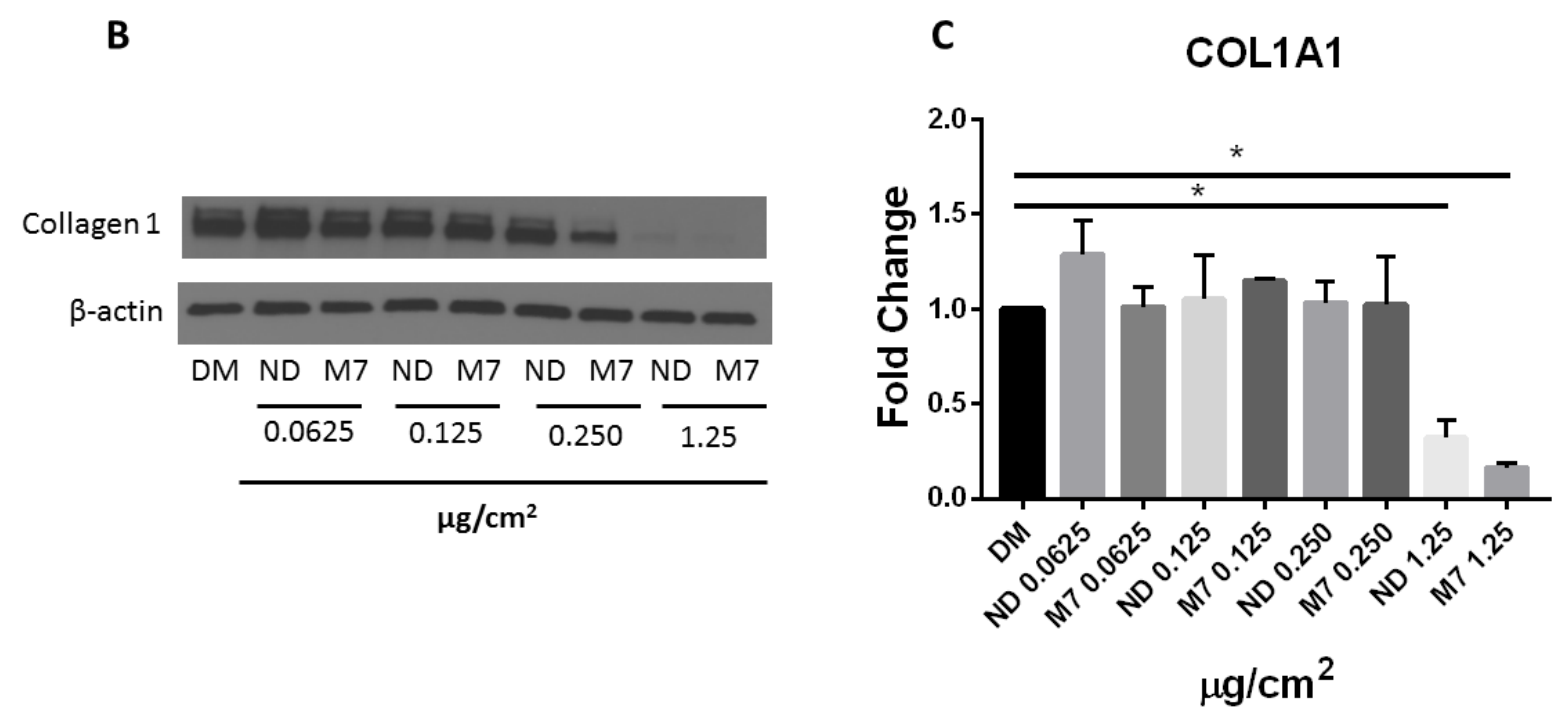

Figure 3.11 Recombinant IL-6 and IL-8 as well as direct exposure to ND-MWCNT and MWCNT-7 alter collagen I expression in WI-38 cells. WI-38 were treated with IL-6, IL-8, or a combination of both over 48 hours. A. Whole cell lysates were resolved by SDS-PAGE on gradient gels and probed for collagen I, suggesting that low concentrations of IL- 6 and IL-8 or in combination induce collagen I production. $\beta$ actin was used as a loading control. B. WI-38 were treated with DM, ND-MWCNT, or MWCNT-7 over a 48 hour period, and results suggested dose-dependent collagen I production by WI-38. C. Additionally, total RNA was collected, cDNA created, and qRT-PCR performed to analyze gene expression changes after CNT exposure from two independent experiments that reflect western blot results $\left({ }^{*}, p<0.05\right)$. 


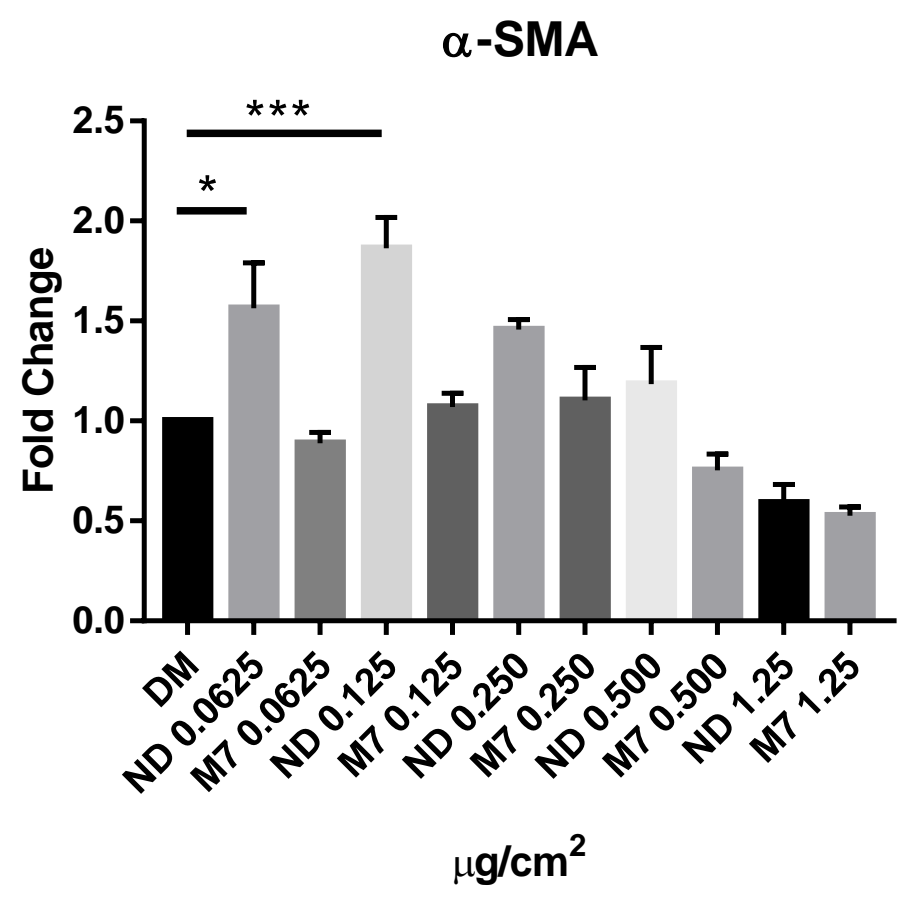

Figure 3.12 Induction of $\alpha$-SMA gene expression by ND-MWCNT and MWCNT-7. WI-38 were exposed to DM, ND-MWCNT, or MWCNT-7 over $48 \mathrm{~h}$. Total RNA was collected, cDNA created, and qRT-PCR performed to analyze gene expression changes after CNT exposure in three independent experiments. $(*, p<0.05, * * *, p<0.001)$. 
Table 3.1 P values associated with ND-MWCNT and MWCNT-7 induced cytotoxicity.

\begin{tabular}{|c|c|c|}
\hline Comparison & Significance & P Values \\
\hline DM vs. ND 2.50 & $*$ & 0.0195 \\
\hline ND 0.125 vs. M7 2.50 & $* * *$ & 0.0009 \\
\hline M7 0.125 vs. ND 2.50 & $* *$ & 0.0107 \\
\hline M7 0.125 vs. M7 2.50 & $*$ & 0.0021 \\
\hline ND 0.250 vs. ND 2.50 & $* *$ & 0.0241 \\
\hline ND 0.250 vs. M7 2.50 & $*$ & 0.0418 \\
\hline M7 0.250 vs. ND 2.50 & $* *$ & 0.0044 \\
\hline M7 0.250 vs. M7 2.50 & $*$ & 0.0469 \\
\hline
\end{tabular}

$(*, \mathrm{p}<0.05, * *, \mathrm{p}<0.01, * * *, \mathrm{p}<0.001)$ 
Table 3.2 P values associated with IL-6 and IL-8 induced changes in WI-38 proliferation

\begin{tabular}{|c|c|c|}
\hline Comparison & Significance & P Values \\
\hline No tx vs. IL-6 "M7" & $*$ & 0.0314 \\
\hline No tx vs. IL-6+IL-8 "ND" IL-6 0.1 ng & $* * *$ & 0.0005 \\
\hline No tx vs. IL-6+IL-8 "M7" & $*$ & 0.0201 \\
\hline No tx vs. IL-6+IL-8 0.1+2.0 ng & $* *$ & 0.0057 \\
\hline IL-6 "ND" vs. IL-6 0.1 ng & $* *$ & 0.0001 \\
\hline IL-6 "ND" vs. IL-6+IL-8 "M7" & $*$ & 0.0028 \\
\hline IL-6 "ND" vs. IL-6+IL-8 0.1+2.0 ng & $* * *$ & 0.0356 \\
\hline IL-6 "M7" vs. IL-6+IL-8 0.1+2.0 ng & $*$ & 0.0222 \\
\hline IL-6+IL-8 "ND" vs. IL-6+IL-8 0.1+2.0 ng & $*$ & 0.0332 \\
\hline
\end{tabular}

$\left({ }^{*}, \mathrm{p}<0.05, * *, \mathrm{p}<0.01, * * *, \mathrm{p}<0.001\right)$ 
Table 3.3 P values associated with WI-38 proliferation after direct exposure to ND-MWCNT or MWCNT-7.

\begin{tabular}{|c|c|c|}
\hline Comparison & Significance & P-values \\
\hline DM vs. ND 0.250 & $*$ & 0.0463 \\
\hline DM vs. ND 1.25 & $* * * *$ & $<0.0001$ \\
\hline DM vs. M7 1.25 & $* * * *$ & $<0.0001$ \\
\hline ND 0.0625 vs. ND 1.25 & $* * * *$ & $<0.0001$ \\
\hline ND 0.0625 vs. M7 1.25 & $* * * *$ & $<0.0001$ \\
\hline M7 0.0625 vs. ND 0.250 & $*$ & 0.0376 \\
\hline M7 0.0625 vs. ND 1.25 & $* * * *$ & $<0.0001$ \\
\hline M7 0.0625 vs. M7 1.25 & $* * * *$ & $<0.0001$ \\
\hline ND 0.125 vs. ND 1.25 & $* * *$ & 0.0002 \\
\hline ND 0.125 vs. M7 1.25 & $* *$ & 0.0018 \\
\hline M7 0.125 vs. ND 1.25 & $* * *$ & 0.0001 \\
\hline M7 0.125 vs. M7 1.25 & $* * *$ & 0.0008 \\
\hline ND 0.250 vs. ND 1.25 & $* * *$ & 0.0007 \\
\hline ND 0.250 vs. M7 1.25 & $* *$ & 0.0061 \\
\hline M7 0.250 vs. ND 1.25 & $* * * *$ & $<0.0001$ \\
\hline M7 0.250 vs. M7 1.25 & $* * *$ & 0.0007 \\
\hline
\end{tabular}

$(*, \mathrm{p}<0.05, * *, \mathrm{p}<0.01, * * *, \mathrm{p}<0.001, * * * *, \mathrm{p}<0.0001)$ 
Chapter 4

General Discussion 


\subsection{Conclusions}

This dissertation presents studies that describe the in vitro bioactivities induced by ND-MWCNT and MWCNT-7 in human lung epithelial and fibroblast cells in the context of their physicochemical properties and the model systems utilized. The unique physicochemical properties of these materials have created new possibilities for industrial and consumer products, as well as increased risk of occupational exposure and toxicity at the nano-bio interface. While numerous functionalized MWCNT are currently in production, our studies focused on the comparison of pristine MWCNT with NDMWCNT, which provided an excellent example to study the role of surface reactivity and presence of defects within the carbon lattice on subsequent bioactivity. ND-MWCNT were determined to have decreased crystallinity in conjunction with increased surface defects (and therefore, biologically-reactive sites). However, results suggested that ND-MWCNT induced an acute inflammatory response in SAEC to a lesser extent than MWCNT-7, but conversely induced greater pro-fibrogenic signaling in fibroblasts, highlighting the need to develop a full understanding of not only how MWCNT physicochemical properties affect cells directly, but of how these properties may impact in vitro assessments compared to in vivo as well. It is possible that the often over-looked properties of pristine and functionalized MWCNT alter their agglomeration, settling, and deposition in submerged in vitro cultures, which is a point of great importance to interpreting in vitro results, especially in future pre-screening studies that simultaneously compare multiple MWCNT. Our studies, which utilized occupationally relevant exposure doses, as well as analysis of the literature, further suggested that the exposure dose(s) utilized in in vitro assessments of insoluble MWCNT are crucial for comparison of results between experiments, other laboratories, and in relation to in vivo and human exposures. This is especially important for the purposes of hazard ranking and regulatory assessment, which often rely upon meta-analysis of numerous completed studies. Taken together, our assessment presented here contributes to improved 
understanding of in vitro-based nanotoxicology, supported by thorough analysis of our results and current in vitro practices in the field.

While the study of MWCNT shares some aspects with general fiber toxicology and ultrafine particle studies, their extremely small diameters and unique composition set them apart from asbestos, silica, ultrafine carbon black, and a myriad of other materials previously studied. They present unique challenges, especially for toxicological study design and predictive in vitro risk assessment. MWCNT are biopersistent fibers, and in the absence of defects, resistant to fully breaking down or dissolving as observed with soluble chemicals and some other nanomaterials [180]. When comparing in vivo and in vitro study methods, it is important to realize that MWCNT behave differently when aerosolized (i.e. a likely real-life occupational exposure scenario) compared to suspension in cell culture media. The size, density, and orientation of MWCNT fibers all contribute to their deposition in the lung when inhaled, such that those with the smallest aerodynamic diameter align parallel to the airflow, bypass the nasopharyngeal and tracheobronchial regions, and settle within the alveolar region [161, 181]. Although not all MWCNT settle within the deep recesses of the alveoli, those that do are of greatest concern since the alveolar region does not have extensive mucociliary clearance nor thick protective mucus found within other regions of the lung [161]. Mimicking this process is difficult in submerged in vitro studies, in which the relative hydrophobicity and surface charge of MWCNT significantly dictates agglomeration behavior and the media itself limits nanomaterial diffusion and settling rates, which may impact observed toxicity $[7,68]$. In our studies, ND-MWCNT and MWCNT-7 appeared to have similar zeta potential measurements in media and PBS (Table 2.1). In 1968, Riddick proposed categories of zeta potential ranges that describe the stability of dispersed particles, suggesting that values between -10 to -15 were within the threshold of agglomeration, thus suggesting that the ND-MWCNT and MWCNT-7 we studied were not in stable suspension [182]. Additionally, although surface charge is important to cellular uptake, it is also driven by agglomerate size, which is also influenced by the total concentration 
of particles available in suspension. Based upon observation, it is likely that agglomeration state and settling varied between exposure doses used in Chapter $\mathbf{2}$ and 3, which may have impacted results and cellular uptake.

The ND-MWCNT used in this study were thoroughly characterized for the first time for the purposes of the manuscript presented in Chapter 2. Results suggested that the ND-MWCNT primarily had pyrrolic incorporation of $\mathrm{N}$ into the carbon lattice (introduced pentagons into hexagonal carbon lattice), which introduced distinct defects and decreased the overall crystallinity of the CNT compared to MWCNT-7 (Figure 2.2, 2.3, and 2.4) Others have also shown that ND-MWCNT are likely to be more brittle and degrade more quickly than pristine MWCNT due to the defects which disrupt the $\mathrm{sp}^{2}$ bonds that give MWCNT their extreme strength and durability. It is important to note the degradation process is not immediate, and therefore, difficult to study in a standard short-term in vitro experiment. Defect sites are also known to be areas of increased reactivity; hence, it may be possible that in the short-term, these particles could appear to be more bioactive than their pristine counterparts in an in vitro setting, and possibly have altered deposition, uptake by macrophage, and persistence in vivo contributing to reduced bioactivity observed in animal studies.

Several in vitro methods were utilized or attempted in order to better understand the bioactivity of ND-MWCNT and MWCNT-7, and presented in this dissertation. SAEC were used to study acute inflammatory responses induced by CNT exposure, which suggested that the two nanofibers elicited in vitro responses of different magnitude and effect. Figure $\mathbf{2 . 5}$ showed a dose response cytotoxicity assay in SAEC suggesting that ND-MWCNT induced limited cell death even at the highest dose $(120 \mu \mathrm{g} / \mathrm{mL})$, while MWCNT-7 caused significant cytotoxicity at the same dose. An exposure dose of $1.2 \mu \mathrm{g} / \mathrm{mL}$ was carried through the rest of the experiments, selected due to its occupational relevance and previous use within the lab $[19,91]$. Results in Figure 2.7. suggest that ND-MWCNT induced sustained ROS production at 6 and $24 \mathrm{~h}$, while MWCNT-7 induced ROS at $6 \mathrm{~h}$ which was resolved at $24 \mathrm{~h}$. 
It is likely that surface defects and reactive sites were present in greater quantity on the surface of NDMWCNT comparatively, thus providing ample opportunity for sustained ROS production. It is important to note that both MWCNT-7 and ND-MWCNT were not found to produce radicals on their own in an acellular system; quite the contrary, it has been shown by multiple groups that pristine and functionalized MWCNT quench ROS effectively in various acellular systems assessed by electron spin resonance spectroscopy $(\operatorname{ESR})[99,100,183]$. However, whether the ROS quenching observed ex vivo is predictive of and translates to MWCNT bioactivity in vivo and in vitro has yet to be thoroughly studied. MWCNT-induced ROS production in vitro has been observed in numerous cell types $[43,91,139,184-$ 186], spurring interest in the role of oxidative stress in MWCNT-related toxicities. We concluded that the ROS induced by ND-MWCNT and MWCNT-7 perhaps acted as signaling molecules that impacted cell cycle progression [137]. ROS can influence the cell cycle through the activation of growth factor receptors and phosphorylation and ubiquitination of cell cycle regulators [138], such as CDK4, which was observed to be elevated in ND-MWCNT exposed SAEC at 6 and $24 \mathrm{~h}$ (similar to production of ROS)

(Figure 2.8). Cell cycle analysis also revealed time- and particle-dependent aberrations primarily within G1 and G2, suggesting that at $24 \mathrm{~h}, \mathrm{ND}-\mathrm{MWCNT}$ and MWCNT-7 were altering cell cycle progression differently, similar to results observed by others showing MWCNT-induced cell cycle aberrations (Table 2.2) $[82,124]$. The use of pan-phospho-tyrosine and phospho-threonine antibodies suggested time and particle-dependent changes in general cell signaling processes, specifically that MWCNT-7 induced a pronounced increase in phospho-tyrosine expression at $6 \mathrm{~h}$ (Figure 2.9). Tyrosine phosphorylation is a widely studied cell signal associated with molecular pathways such as mitogen-activated protein kinase (MAPK) pathways, Jak/STAT pathways, and phosphotidylinoside 3-kinase (PI3K) [126], all of which have been linked to MWCNT exposure $[19,41,187-189]$. Together, the results ultimately led us to conclude that the physicochemical properties of ND-MWCNT and MWCNT-7 were interacting with and modulating alternative in vitro responses in SAEC. 
While useful to know that ND-MWCNT and MWCNT-7 induced an acute inflammatory response in epithelial cells, we wished to build upon these results and aim to develop a predictive model of MWCNT-induced pulmonary fibrosis in vitro. In vivo studies suggested that pulmonary fibrosis, which is often irreversible and incurable at diagnosis in humans, is likely one of the greatest adverse outcomes associated with MWCNT exposure and therefore of pressing need to study thoroughly. Within the alveolar region, epithelial cells create a barrier against foreign material and produce surfactant that aids in gas exchange. Underneath this layer, resident fibroblasts are found within the interstitial space, ready to act in the event that the epithelium is damaged. Fibrosis occurs when these wound repair processes become dysregulated and fibroblasts are unable to stop proliferating and depositing ECM, increasingly believed to be the result of unresolved epithelial injury and dysregulated epithelial-derived signaling [146]. Therefore, we attempted to develop an epithelial-fibroblast co-culture model aimed to study the paracrine and autocrine signaling at play which leads to this response.

Numerous attempts to develop a cohesive co-culture model with SAEC or BEAS-2B epithelial cells and WI-38 fibroblasts using Transwells ${ }^{\circledR}$ were made to limited avail and are presented at length in Appendix I for further reference. Limiting issues pertained to cell media compatibility, cell viability, cell phenotype, and practical feasibility to maintain both cell types together long enough to observe profibrogenic signaling. Due to persistent and insurmountable difficulties with the SAEC in the presence of WI-38, BEAS-2B bronchial epithelial cells were selected as an alternative epithelial model due to their hardiness, widespread use in in vitro toxicological assessments, and relevance to the study of cellular airway injury. Each growth condition and cell line selection impacted cell marker expression (and this without any exposure to MWCNT), providing one possible reason for why in vitro results often differ between mono- and co-culture assessment (Supplemental Figure 1.1, 1.2, 1.5, 1.6, 1.7, 1.8). In these studies, CCL2, a chemokine known to contribute to pulmonary inflammation and fibrosis, only differed between growth conditions, but not between DM and MWCNT-7 exposed cells (Supplemental Figures 
1.3, 1.4, and 1.9). Together, these results suggested that 1) growth conditions and cell line could significantly impact chemokine production and 2) CCL2 was unlikely to be an ideal chemokine for study in MWCNT-7 induced fibrosis in this in vitro system.

Upon closer inspection while attempting to figure out why CCL2 ELISA results were not in concordance with previously achieved results in our lab and by others [76, 91, 172], especially within monoculture SAEC, it became clear that perhaps the exposure dose metric used could inadvertently impact the results. Unless the surface area of the culture dish is taken into consideration during exposure dosing, one can introduce over a 2-fold difference in MWCNT available for deposition on cells comparing between a Transwell $^{\circledast}$ and monoculture dish. CCL2 ELISAs previously carried out in the lab in SAEC monoculture with an exposure dose of $1.2 \mu \mathrm{g} / \mathrm{mL}$ were carried out in 24 well dishes with a surface area of $1.9 \mathrm{~cm}^{2}$ with $0.5 \mathrm{~mL}$ media used during exposure, which when converted into a surface area based dose as shown in the Introduction, is equivalent to $0.32 \mu \mathrm{g} / \mathrm{cm}^{2}$. Using similar logic, a 6 well Transwell ${ }^{\circledast}$ has a surface area of $4.67 \mathrm{~cm}^{2}$ compared to a standard 6 well dish with a surface of $9.5 \mathrm{~cm}^{2}$. Since exposures of $1.2 \mu \mathrm{g} / \mathrm{mL}$ were carried out in $1.5 \mathrm{~mL}$ of media in both Transwells ${ }^{\circledR}$ and monoculture, SAEC on Transwells ${ }^{\circledR}$ received a surface area-based exposure dose of $0.38 \mu \mathrm{g} / \mathrm{cm}^{2}$ while concurrent monoculture experiments received $0.18 \mu \mathrm{g} / \mathrm{cm}^{2}$, which could explain the less robust CCL2 production observed in SAEC monoculture here. Since the co-culture system appeared to unreasonably stress the cells at well, that likely also resulted in less robust CCL2 production (Supplemental Figure 1.3). In order to normalize dose between mono- and co-culture and directly to in vivo exposure, we converted an 80 $\mu \mathrm{g}$ in vivo dose previously shown to induce fibrosis in mice $[40,77]$ to a surface area based dose for both Transwell ${ }^{\circledR}$ and monoculture exposures, which was $0.16 \mu \mathrm{g} / \mathrm{cm}^{2}$ and therefore 2 -fold lower than that previously used in the lab. Supplemental Figure 1.4. and 1.9. suggested that this dose, while occupationally relevant and directly relevant to in vivo exposure, elicited limited results in vitro in both SAEC and BEAS-2B. Therefore, this work demonstrated how crucial it is to understand dosing between 
culture systems, and also that perhaps occupationally relevant doses may not always elicit as robust results in vitro.

Despite the difficulties experienced within our lab with epithelial-fibroblast co-culture, others have published similar co-culture systems using alternative cell lines (often requiring the use of lab animals or humans to harvest the cells) primarily for the purpose of studying conditions such as asthma, chronic bronchitis [21], pulmonary injury [18, 22, 32, 190], and pulmonary fibrosis [20,191]. At this point in time, it appears that epithelial-fibroblast co-culture systems utilizing well-known and widely available immortalized cells for the purposes of predictive or mechanistic toxicology assessment are not generally available. It will also remain important to understand the impact of the model system itself on cellular responses to better understand results, especially for toxicology assessment and comparisons between mono- and co-culture models.

A conditioned media approach was subsequently utilized as an alternative approach to coculture to reduce cell-cell compatibility issues and find a dose that would be more bioactive than those used in co-culture. As direct exposure to SAEC media was found to be highly toxic to WI-38 in early experiments, BEAS-2B, a hardier alternative epithelial cell line, was utilized in these studies. Experiments were designed such that BEAS-2B were exposed to ND-MWCNT or MWCNT-7 over $24 \mathrm{~h}$, media was collected and centrifuged to remove extraneous CNT and debris, and added 1:1 to WI-38 for $48 \mathrm{~h}$ (Supplemental Figure 2.4). Although BEAS-2B engulfed ND-MWCNT and MWCNT-7 (Supplemental Figures 2.2 and 2.3), WI-38 exposed to conditioned media were shown to have limited to no presence of CNT after $48 \mathrm{~h}$ as assessed by TEM (Supplemental Figure 2.5). qRT-PCR results suggested that 9.6 $\mu \mathrm{g} / \mathrm{mL}\left(1.37 \mu \mathrm{g} / \mathrm{cm}^{2}\right)$ ND-MWCNT significantly increased PDGF-A gene expression in BEAS-2B, which has been closely implicated in pulmonary fibrosis, as well as pristine and functionalized MWCNT exposure $[68,76,192-194]$ (Supplemental Figure 2.7). However, protein expression of PDGF-AA did not follow suit at the same dose (Supplemental Figure 2.8). Several preliminary proliferation assays in WI-38 
exposed to conditioned media suggested no significant change, regardless of cell density. We then asked why BEAS-2B exposed to 4.8 and $9.6 \mu \mathrm{g} / \mathrm{mL}\left(1.25\right.$ and $\left.2.50 \mu \mathrm{g} / \mathrm{cm}^{2}\right)$ ND-MWCNT and MWCNT-7 released IL-13, IL-6, and IL-8, and likely other cell signaling mediators (Figure 3.4), while BEAS-2B conditioned media had limited effects on WI-38 exposed over $48 \mathrm{~h}$. Small ancillary experiments suggested that the media type, duration of exposure, nor cell densities played a role in the WI-38 results. Much like comparison between the Transwell ${ }^{\circledR}$ and monoculture models, we determined that changes in the surface area of various monoculture cell dishes could alter the exposure dose over 2-fold. In essence, every time a different dish size was used, which is clearly quite common in research, the exposure dose also changed; it is critical to note that a majority of early and relatively current nanotoxicology assessments were performed using concentration based dosing, which may have interesting implications for overall data interpretation.

The use of conditioned media, as discussed in the Introduction, requires that cells respond with the same magnitude each time to each particle and each dose, with protein production occurring such that their half-lives are the same each time in the conditioned media applied to the WI-38. Hence, after performing an additional PDGF-AA ELISA using BEAS-2B media (Supplemental Figure 2.8) and qRT-PCR for genes known to be associated with fibrosis and fibroblast activation including PDGF-1, TGFB, VIM, and $\alpha$-SMA in WI-38 without clear-cut results even after using conditioned media from BEAS-2B exposed to 1.25 or $2.50 \mu \mathrm{g} / \mathrm{cm}^{2}$ (Supplemental Figure 2.9), additional alternative approaches were assessed. It became clear that before we could focus on comparing the bioactivity of ND-MWCNT and MWCNT-7, finding a reliable model system was of greater precedence.

Two additional methods were developed and utilized in Chapter $\mathbf{3}$ in order to address the indirect and direct effects of ND-MWCNT and MWCNT-7 on pro-fibrogenic processes in WI-38 concurrently. WI-38 were exposed to biologically relevant concentrations of exogeneous IL-6 and IL-8 directly derived from the $\mathrm{pg} / \mathrm{mL}$ concentrations found in BEAS2-2B conditioned media after $24 \mathrm{~h}$ 
exposure to 1.25 or $2.50 \mu \mathrm{g} / \mathrm{cm}^{2}$ ND-MWCNT or MWCNT-7 (Figure 3.4). WI-38 were additionally exposed to a range of exposure doses from 0.0625 to $1.25 \mu \mathrm{g} / \mathrm{cm}^{2}$ ND-MWCNT or MWCNT-7 directly, which correlate with 2.8 month and 4.6 year occupational exposure in humans calculated using values as previously described $[40,46]$. These in vitro methods were employed to circumvent the issues we faced with co-culture and conditioned media approaches, while still addressing specific epithelialfibroblast communication and the effects of direct exposure to fibroblasts likely to occur to some extent in vivo. IL-6 and IL-8 administered for $48 \mathrm{~h}$ at biologically relevant concentrations and the manufacturer's suggested dose had limited effect on WI-38 viability or total cell number (Figure 3.9 A, B), but IL-6 and IL6 in combination with IL-8 appeared to significantly reduce WI-38 proliferation (Figure 3.10 A). While IL-6 and IL-8 have traditionally been associated with supporting fibrogenic processes in vivo and in vitro $[159,167,168,195,196]$, it is especially important to note the pleiotropic nature of IL-6 and the study designs traditionally employed for this purpose $[156,197,198]$. A series of papers by Moodley et al. showed that primary lines of normal fibroblasts and IPF fibroblasts derived from patients exhibited alternative responses to IL-6, such that IL-6 inhibited proliferation of normal fibroblasts and induced apoptosis with exact opposite effects on IPF fibroblasts $[197,198]$. This work suggested that signaling pathways in normal vs. activated disease-state fibroblasts are altered, with IPF signaling pathways supporting increased collagen deposition, increased proliferation, and resistance to apoptosis typically associated with fibrosis. It may be possible that the WI-38, which are considered normal fibroblasts, lacked the necessary phenotype prone to true fibrogenesis (especially within $48 \mathrm{~h}$ ), and therefore, would not exhibit robust results suggestive of fibrotic signaling despite exogenous exposure to IL-6 and IL-8. Additionally, cell-cell communication typically does not occur in the absence of other mediators and signaling molecules. In vivo, MWCNT-7 have been shown to induce production of TNF- $\alpha$, IL-1 $\alpha, I L-1 \beta$, and IL- 6 in BAL fluid of mice exposed to a $40 \mu \mathrm{g}$ exposure dose and subsequent expression of TGF $\beta$ and PDGF-A in fibrotic lung tissue, suggesting that perhaps IL-6 is likely not the primary singular 
driver behind MWCNT-induced pulmonary fibrosis as well, but requires the presence of other cytokines to support fibrogenesis [76].

WI-38 directly exposed to CNT in monoculture, while the most basic of the in vitro methods utilized, achieved results more closely suggestive of pro-fibrogenic signaling. However, the ND-MWCNT appeared to be more bioactive than MWCNT-7, which was not in concordance with the limited published information on similar ND-MWCNT nor an unpublished in vivo study using the ND-MWCNT studied here. Collagen I production appeared more robust in WI-38 directly exposed to CNT compared to IL-6 or IL-8, especially those exposed to 0.0625 or $0.125 \mu \mathrm{g} / \mathrm{cm}^{2}$ ND-MWCNT compared to control (Figure 3.11 A, B), which was further supported by qRT-PCR (Figure 3.11 C). Gene expression for $\alpha$-SMA also generally followed collagen I production, which is suggestive of early stages of fibroblast-tomyofibroblast activation (Figure 3.12). While we did not observe increased WI-38 proliferation after direct exposure to ND-MWCNT or MWCNT-7 regardless of exposure dose (Figure 3.10 B), this may be related to the cell line and dose range. Without any established, widely held guidelines governing in vitro exposure doses, their correlation to real-life exposures, or their relevance to predicting potential toxicity, it can be challenging to compare between studies, many of which claim to use occupationally relevant doses (even those upwards of $100 \mu \mathrm{g} / \mathrm{mL}$, which equate to quite high and variable surface area based doses nearly impossible to translate in vivo, let alone in humans lest they be on the job for centuries). Manuscripts suggesting that MWCNT induced proliferation in vitro used doses up to 30 $\mu \mathrm{g} / \mathrm{cm}^{2}$, far higher than what we used $[43,150]$, while Mishra et al. presented one of the only available MWCNT in vitro studies in fibroblasts using quite low exposure doses between 0.002 and $0.6 \mu \mathrm{g} / \mathrm{cm}^{2}$ (roughly 1.8 days to 2.2 years for human exposure) [110]. However, only the lowest doses ranging between 0.002 and $0.06 \mu \mathrm{g} / \mathrm{cm}^{2}$ induced proliferation in CRL1490 fibroblasts. It may be possible that the doses selected in our study were simply not within the "window of opportunity" noted between extremely low and quite high exposures in vitro. If so, that poses interesting questions for the future of 
in vitro toxicology, especially as risk assessment shifts to increased reliance on in vitro models as part of a proposed tiered approach $[5,11]$.

The work presented here addressed the strong need identified for development of in vitro models that provide results which are in concordance with in vivo and human exposures, especially with the urge to implement "safety-by-design" to guide industrial production prior to widespread use and potential human exposure. Although in vitro models have provided a mixed bag of data for MWCNT assessment, primarily due to lack of standardization between laboratories in assays, exposure dosing, and adequate model systems capable of addressing the potential long-term impact of MWCNT exposure, our results helped bring nanotoxicology one step closer to developing such models. It is also important to sharpen what we as toxicologists want from in vitro studies - in-depth assessment of molecular mechanism or high-throughput prediction of toxicity. While both are possible, it is unlikely the same in vitro model will adequately fulfill these needs in the future. Although the use of "normal" cells such as SAEC or BEAS-2B were used here for the purpose of assessing MWCNT-7 and ND-MWCNTinduced acute inflammatory responses in pulmonary epithelium, in vitro models of fibrosis that are more reflective of the disease state and its progression for the purpose of understanding the inherent long-term risks associated with MWCNT exposure may be hampered by the use of "normal" fibroblasts in short-term exposures, and will therefore require further study.

The results presented here are valuable because they draw attention to the issue of in vitro and in vivo concordance in toxicology research. If regulatory agencies plan to rely upon high-throughput in vitro methods to rank nanomaterials by bioactivity, provide QSAR data for predictive in silico modeling, and triage materials for further in vivo study, it is essential to ensure that the model systems utilized are capable of logically and accurately reflecting in vivo responses. The unpublished in vivo aspiration study utilizing ND-MWCNT at occupationally relevant exposure doses in mice suggested that ND-MWCNT were expected to have similar alveolar deposition to MWCNT-7, but were shown to elicit a dampened 
inflammatory and fibrotic response comparatively. It was concluded that on a mass basis, ND-MWCNT were therefore less toxic. Results presented in WI-38 monoculture experiments, however, suggested that the ND-MWCNT were potentially more bioactive. Thus far, few studies have been carried out comparing a panel of functionalized MWCNT (or even two) in vitro; those that have typically used in vitro models of macrophage or epithelial cells, extrapolating those results to possible fibrogenesis without directly showing it in vitro in fibroblasts. Additionally, limited information is available on how functionalization of MWCNT impacts the relationships between physicochemical characteristics, toxicity, and dosimetry in vitro. The behavior and characteristics of a dry nanomaterial aerosolized in a factory is incomparable to the same material suspended in aqueous solution, and without an accurate ISDD model available for nanofibers including MWCNT, limited experimental data is available on how MWCNT functionalization alters deposition, availability, and the actual exposure dose cells receive in vitro (which may have differed between ND-MWCNT and MWCNT-7 in these studies). ND-MWCNT, due to the known surface defects present within their structure, likely have more reactive sites available that can enhance cellular responses in vitro, and perhaps alter their activity in vivo. Perhaps in vivo, ND-MWCNT, due to their surface reactivity, are more likely to be engulfed by macrophage and other immune cells, therefore limiting the number of fibers that reach the alveolar epithelium, subsequently decreasing the number of fibers that can pass into the interstitial space. Another often overlooked consideration is MWCNT degradation and debundling of agglomerates, which is likely to occur in vivo. Zhao et al. showed complete degradation of ND-MWCNT in the presence of HRP and $\mathrm{H}_{2} \mathrm{O}_{2}$ over 80 days, suggesting that HRP can more easily bind to defect sites nitrogen introduces into the graphene lattice [153]. While a direct comparison cannot be made between our studies and this work, it is possible that in vivo, NDMWCNT may be broken down more efficiently than pristine MWCNT into less toxic degradation products in macrophage, which we obviously could not model in a fibroblast monoculture system. In order to more accurately reflect the richness of an in vivo exposure and better understand cell-cell 
communication, future studies utilizing human macrophage, epithelial cells, and carefully selected fibroblasts in culture together could provide great insight into the signaling processes that elegantly orchestrate pulmonary inflammation and pro-fibrogenic signaling.

In closing, this work suggests that the risk assessment for MWCNT and functionalized MWCNT may not be as straightforward as originally assumed. As one of the most widely studied nanomaterials with high potential for use in industrial, consumer, and biomedical products, it is essential to understand the possible risks to human and environmental health. In order to effectively address these risks, the model systems used to study these materials as well as the physicochemical properties of MWCNT must be carefully considered while analyzing results and developing hazard ranking parameters that may be used one day to predict the toxicity of other nanomaterials. It is important to note that while great strides have been made, it is unlikely that in vitro systems currently in use could replace the use of in vivo models at this point in time.

The quest for accurate, predictive in vitro models for nanotoxicology assessment is still on-going, and will likely continue on in the future as the field moves away from reliance on in vivo experimentation. The work presented here is one piece of the puzzle; having a clear understanding of the possibilities and limitations of in vitro study will allow researchers in the future to refine and develop new hypotheses that will move us towards models that will one day enable toxicologists to make regulatory decisions that create safer, healthier workplaces. Overall, we showed that ND-MWCNT and MWCNT-7 are capable of inducing acute inflammatory responses in SAEC and BEAS-2B lung epithelial cells and provided an assessment of in vitro methodologies that can be employed to study the complexities of MWCNT-induced pulmonary fibrosis, with especial attention to the impact physicochemical properties have on bioactivity and in vitro risk assessment. We found that it likely that the presence of surface defects in MWCNT plays a key role in predicting bioactivity, and will require further elucidation in the future using a variety of in vitro and in vivo methods. We also anticipate that 
the future will hold solutions to the limitations of today, which is only possible when we communicate our full findings to other scientists, not as failures, but simply as obstacles that can be collectively overcome. 


\section{Appendix I}

Supplemental Figures, Results, and Methods 


\section{Supplemental Figures:}

SAEC Pre Co-Culture

SAEC WI-38 Co-Culture
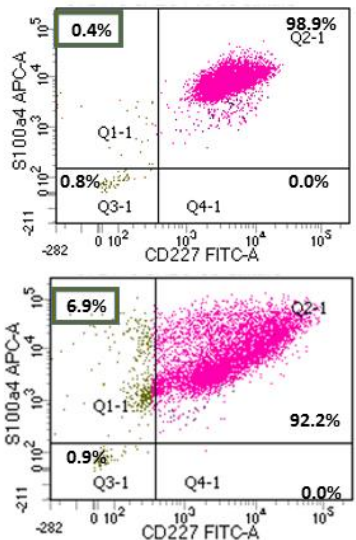

SAEC

Co-Culture Without WI-38

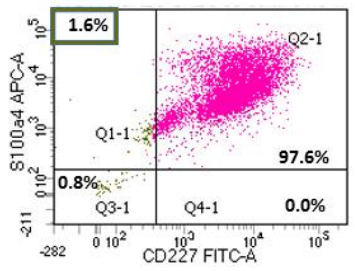

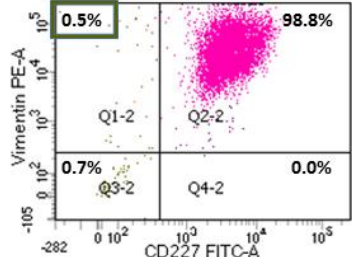
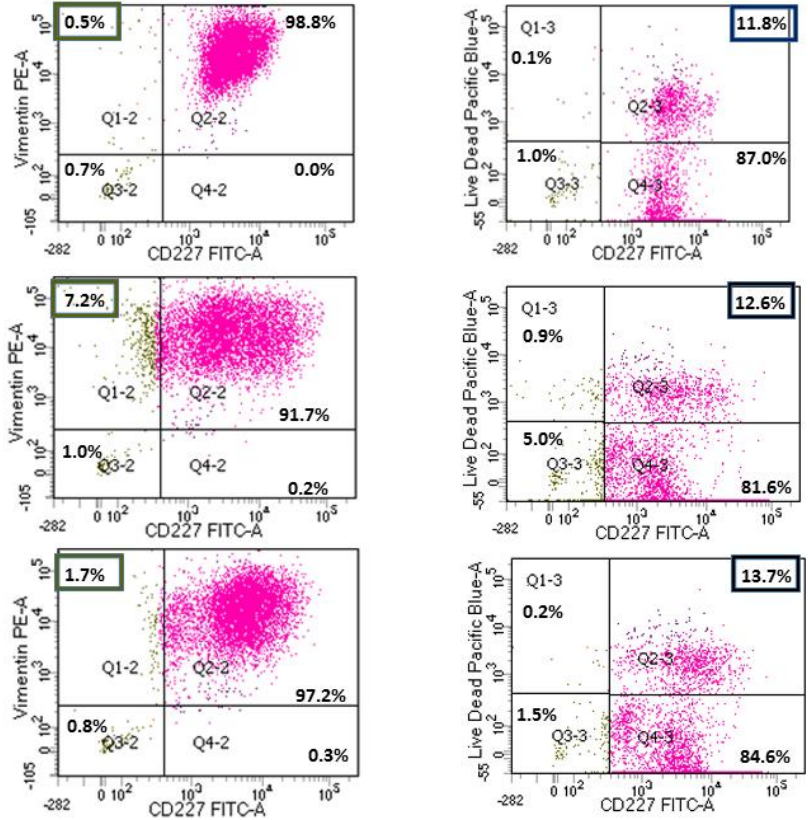

Supplemental Figure 1.1. SAEC expression of cell phenotype markers changes with culture conditions

alone. Flow cytometry was used to monitor changes in cell phenotype markers vimentin, S100A4, and CD-227 as well as cell death by live/dead staining in SAEC seeded on Transwells ${ }^{\circledR}$ before co-culture, after 48 hours in co-culture, and after 48 hours alone on Transwells ${ }^{\circledR}$ with WI-38 EMEM media only below. Results suggested that over $10 \%$ of SAEC were dying simply by being on the Transwells ${ }^{\circledR}$, and co-culture conditions increased the percentage of SAEC expressing vimentin and S100A4. 

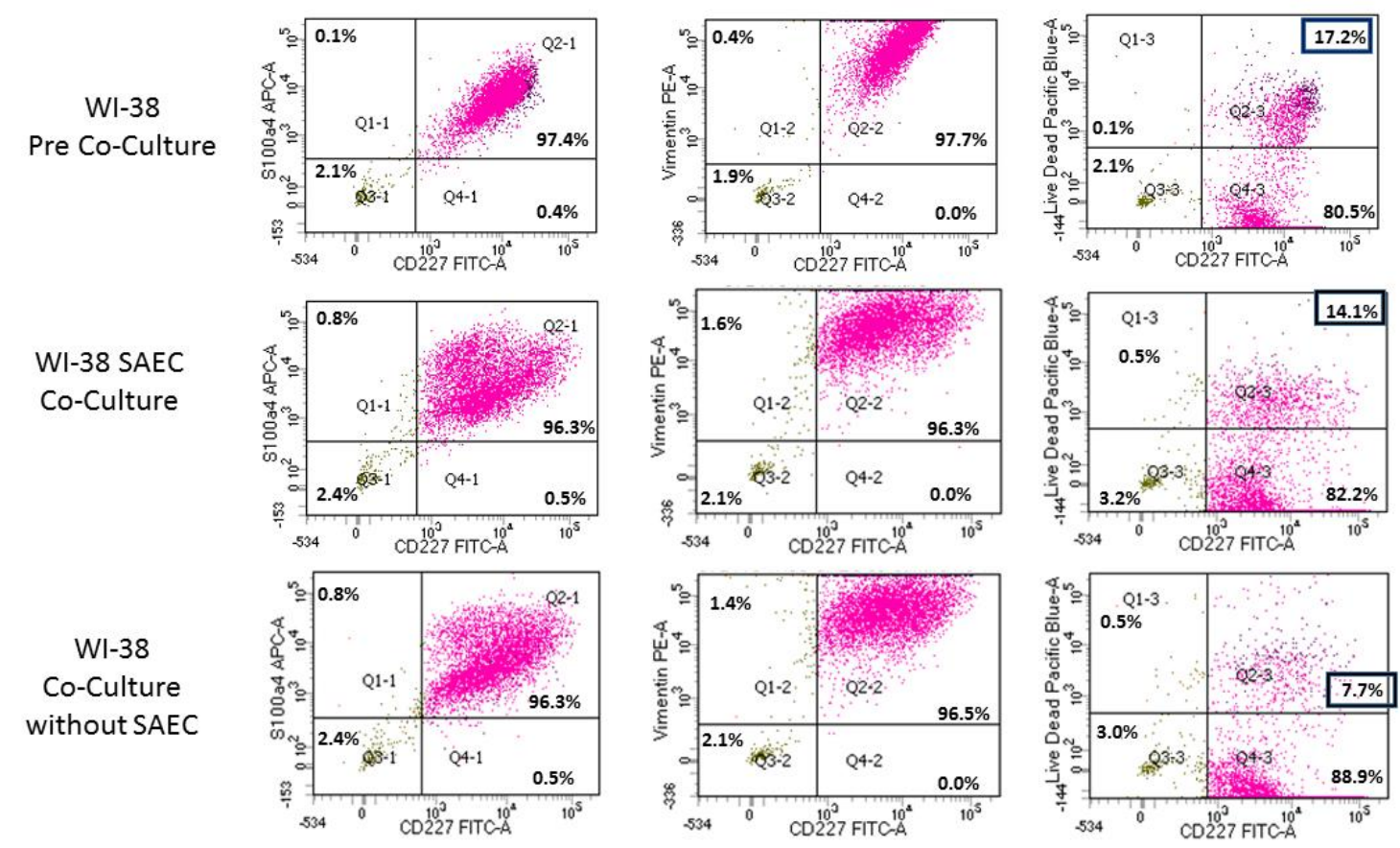

Supplemental Figure 1.2. WI-38 expression of cell phenotype markers changes with culture conditions

alone. Flow cytometry was used to monitor changes in cell phenotype markers vimentin, S100A4, and CD-227 as well as cell death by live/dead staining in WI-38 seeded beneath Transwells ${ }^{\circledR}$ before coculture, after 48 hours in co-culture, and after 48 hours alone beneath Transwells ${ }^{\circledR}$ with SAEC media only above. Results suggested that WI-38 were dying simply by being within the system, but limited phenotype marker changes were noted. 


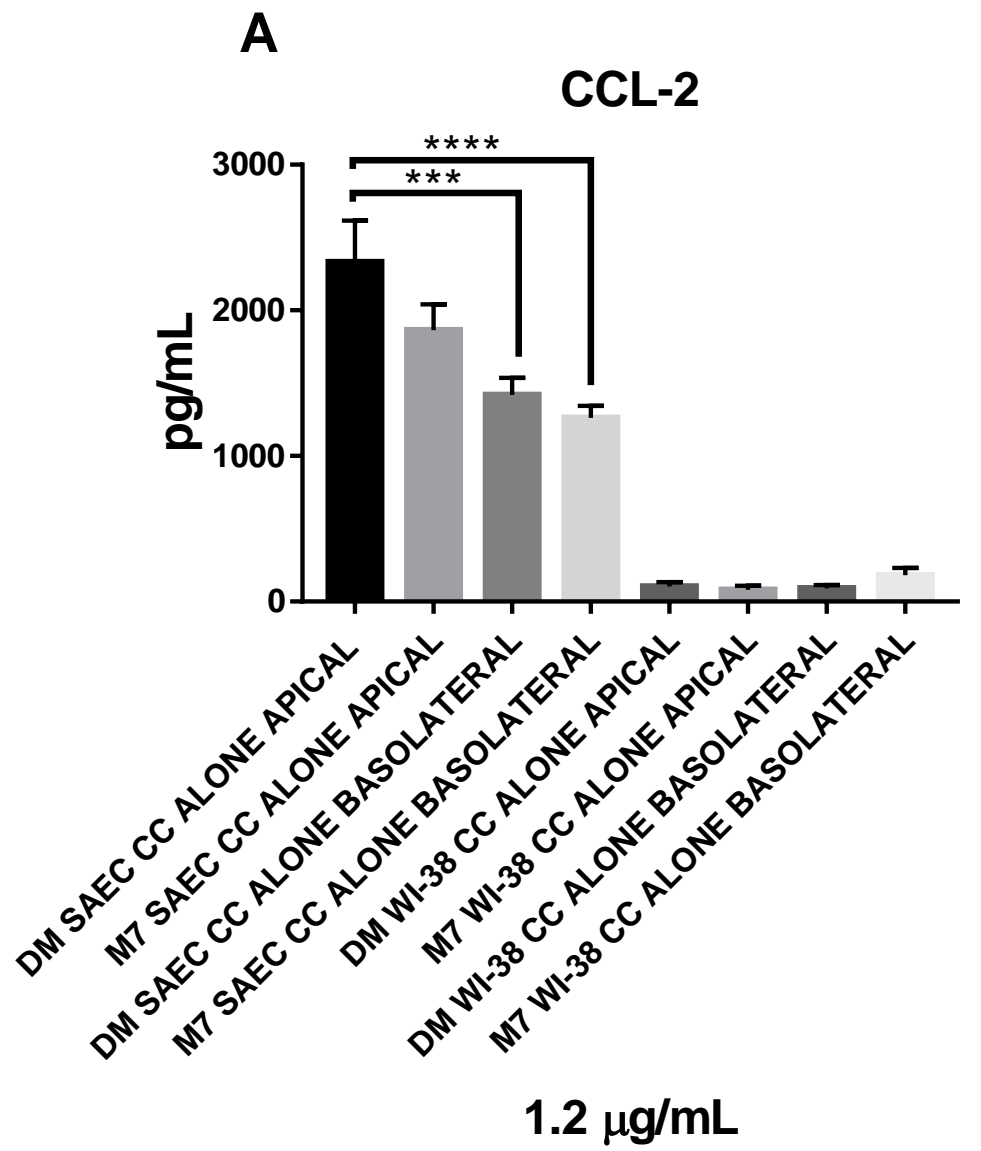

B

CCL-2

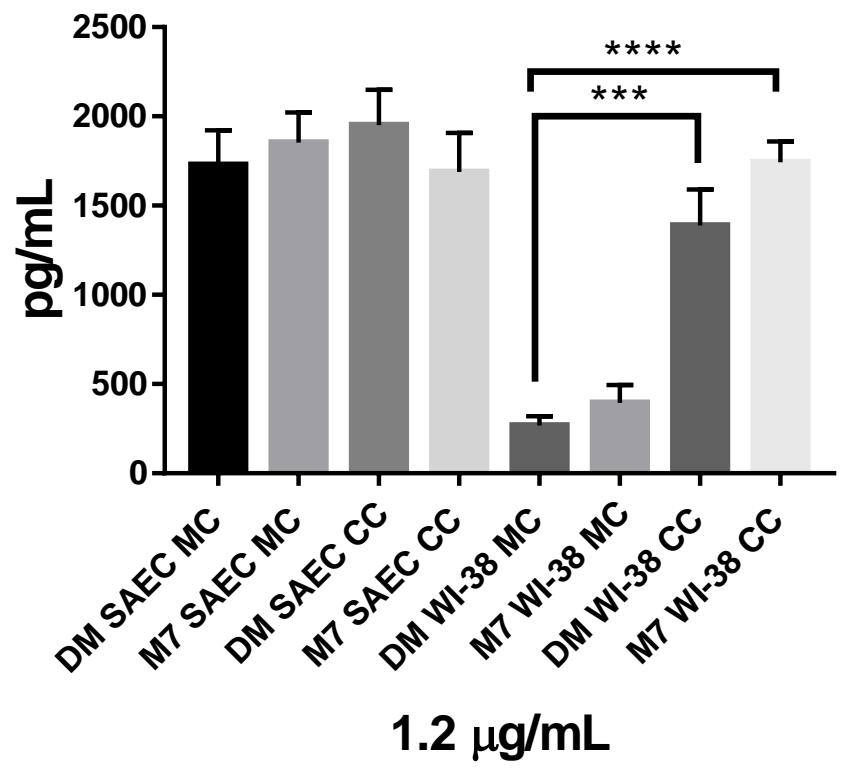




\section{Supplemental Figure 1.3. CCL2 production is altered in SAEC and WI-38 under various co-culture}

conditions. SAEC and WI-38 were seeded in monoculture, in co-culture, and alone in the apical or basolateral chamber of a Transwell ${ }^{\circledR}$ respectively. Media samples from the respective wells were collected 24 hours after exposure to $1.2 \mu \mathrm{g} / \mathrm{mL}$ DM or MWCNT-7, spun down to remove debris and extraneous MWCNT-7, and assayed by ELISA in two independent experiments. Results suggest that there are no significant changes in CCL2 between DM and MWCNT-7 treated samples within the same

culture conditions (A, B). However, the difference between apical and basolateral wells of SAEC alone suggested that CCL2 passed through the $\operatorname{Transwe}^{\circledR}(\mathbf{A})$ and the difference between WI-38 in monoculture and co-culture was also significant (B). One-Way ANOVA statistical testing was carried out followed by Tukey's test for multiple comparisons ( $\left.{ }^{* * *}, \mathrm{p}<0.001, * * * *, p<0.0001\right)$ 


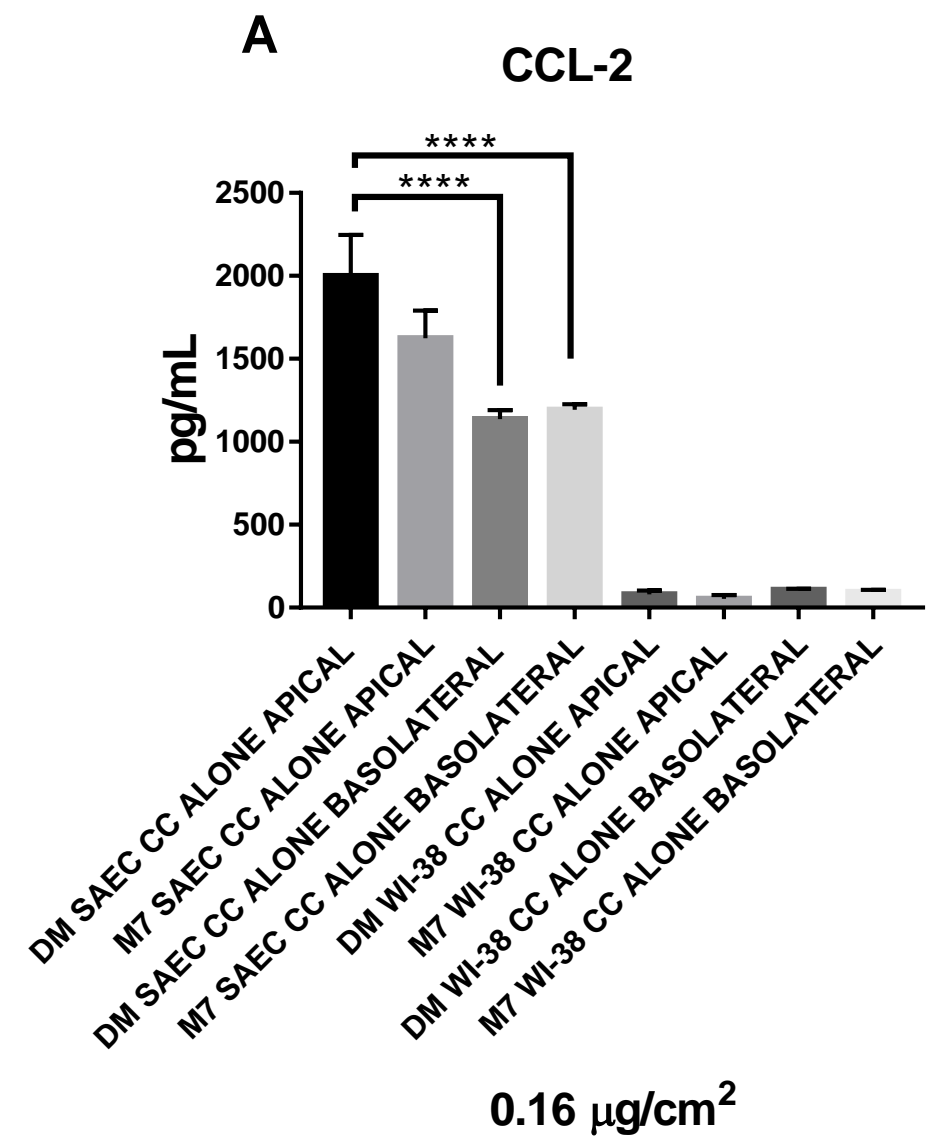

B

CCL-2

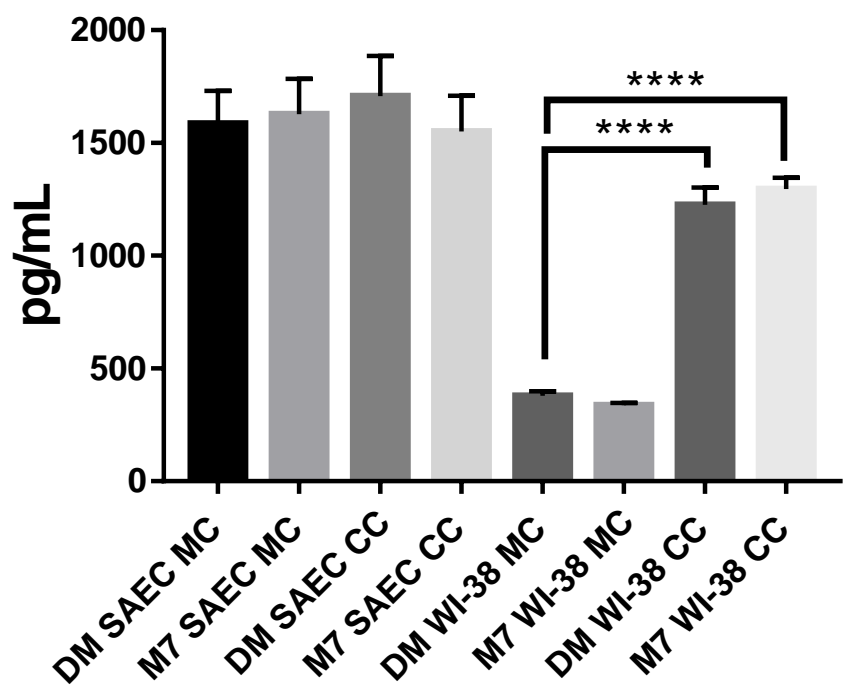

$0.16 \mu \mathrm{g} / \mathrm{cm}^{2}$ 
Supplemental Figure 1.4. Changes in dosimetry between monoculture and co-culture samples yield no significant changes in CCL2 production in SAEC and WI-38 within treatment groups. SAEC and WI-38

were seeded as described above and media was collected from the apical chamber of all treatment groups for assessment by ELISA for CCL2 in three independent experiments. In an attempt to normalize the total $\mu \mathrm{g}$ dose SAEC would receive, doses were recalculated to an occupationally relevant 0.16 $\mu \mathrm{g} / \mathrm{cm}^{2}$ to reflect differences in cell growth surface area between Transwells ${ }^{\circledR}$ and monoculture 6-well dishes. Results suggest that there are no significant changes in CCL2 between DM and MWCNT-7 treated samples within the same culture conditions as assessed by One-Way ANOVA between all means. However, the difference between apical and basolateral wells of SAEC alone suggested that CCL2 passed through the Transwells ${ }^{\circledR}(\mathbf{A})$ and the difference between WI-38 in monoculture and co-culture was also significant $(\mathrm{B})(* * *, \mathrm{p}<0.001, * * * *, \mathrm{P}<0.0001)$. 




Supplemental Figure 1.5. BEAS-2B in DMEM F-12 alter expression of cell phenotype markers changes

with culture conditions alone. Flow cytometry was used to monitor changes in cell phenotype markers vimentin, S100A4, and CD-227 as well as cell death by live/dead staining in BEAS-2B in DMEM-F12 media seeded on Transwells ${ }^{\circledR}$ before co-culture, after 72 hours in co-culture, and after 72 hours alone on Transwells ${ }^{\circledR}$ with WI-38 EMEM media only below. Results suggested that limited cell death occurred, but conditions significantly altered the percentage of BEAS-2B co-expressing S100A4 and vimentin and altered co-expression of S100A4, vimentin, and CD-227 expression in co-culture and co-culture without the presence of WI-38. 
WI-38

Pre Co-Culture

WI-38 Beas-2B

Co-Culture

WI-38 Co-Culture without Beas-2B
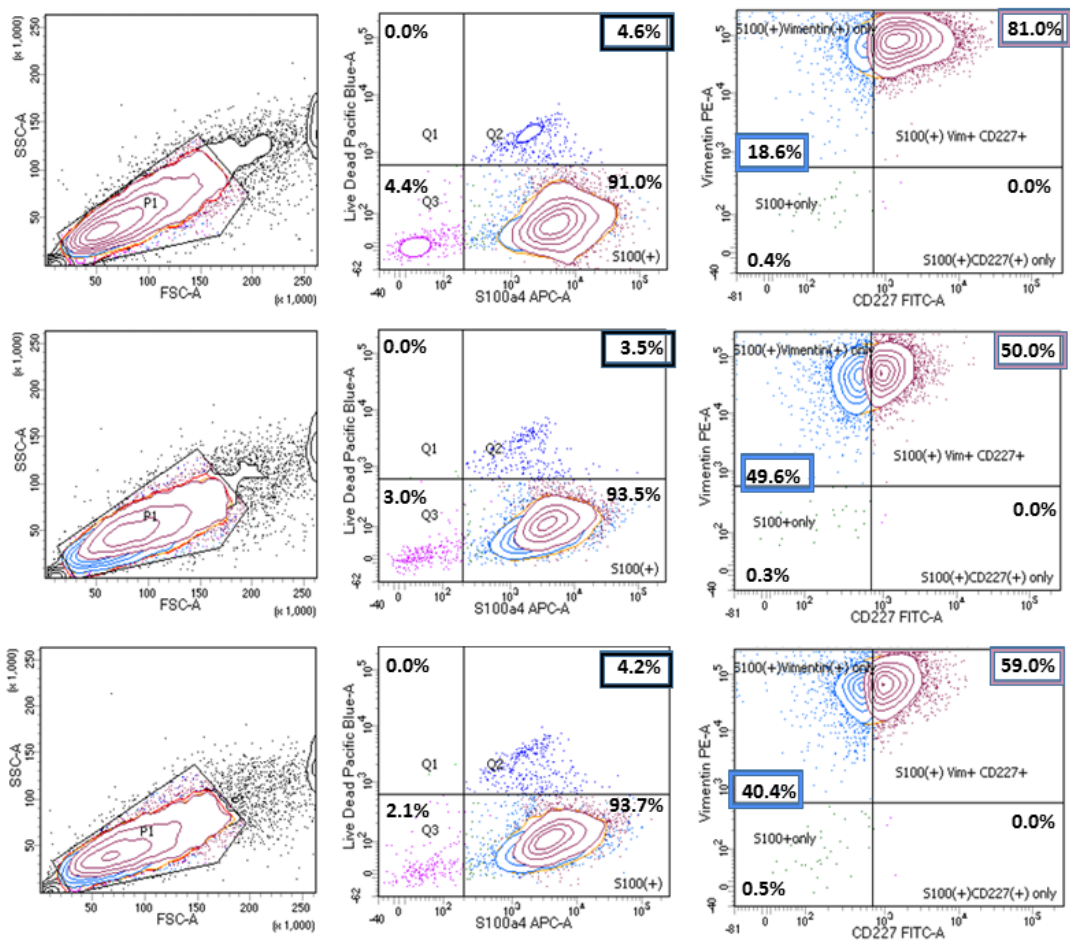

Supplemental Figure 1.6. WI-38 in EMEM alter expression of cell phenotype markers changes with

culture conditions alone. Flow cytometry was used to monitor changes in cell phenotype markers vimentin, S100A4, and CD-227 as well as cell death by live/dead staining in WI-38 in EMEM media seeded beneath Transwells ${ }^{\circledR}$ before co-culture, after 72 hours in co-culture, and after 72 hours alone beneath Transwells ${ }^{\circledR}$ with BEAS-2B DMEM-F12 media only above. Results suggested that limited cell death occurred, but conditions increased the percentage of WI-38 co-expressing S100A4 and vimentin and decreased co-expression of S100A4, vimentin, and CD-227 expression in co-culture and co-culture without the presence of BEAS-2B. 


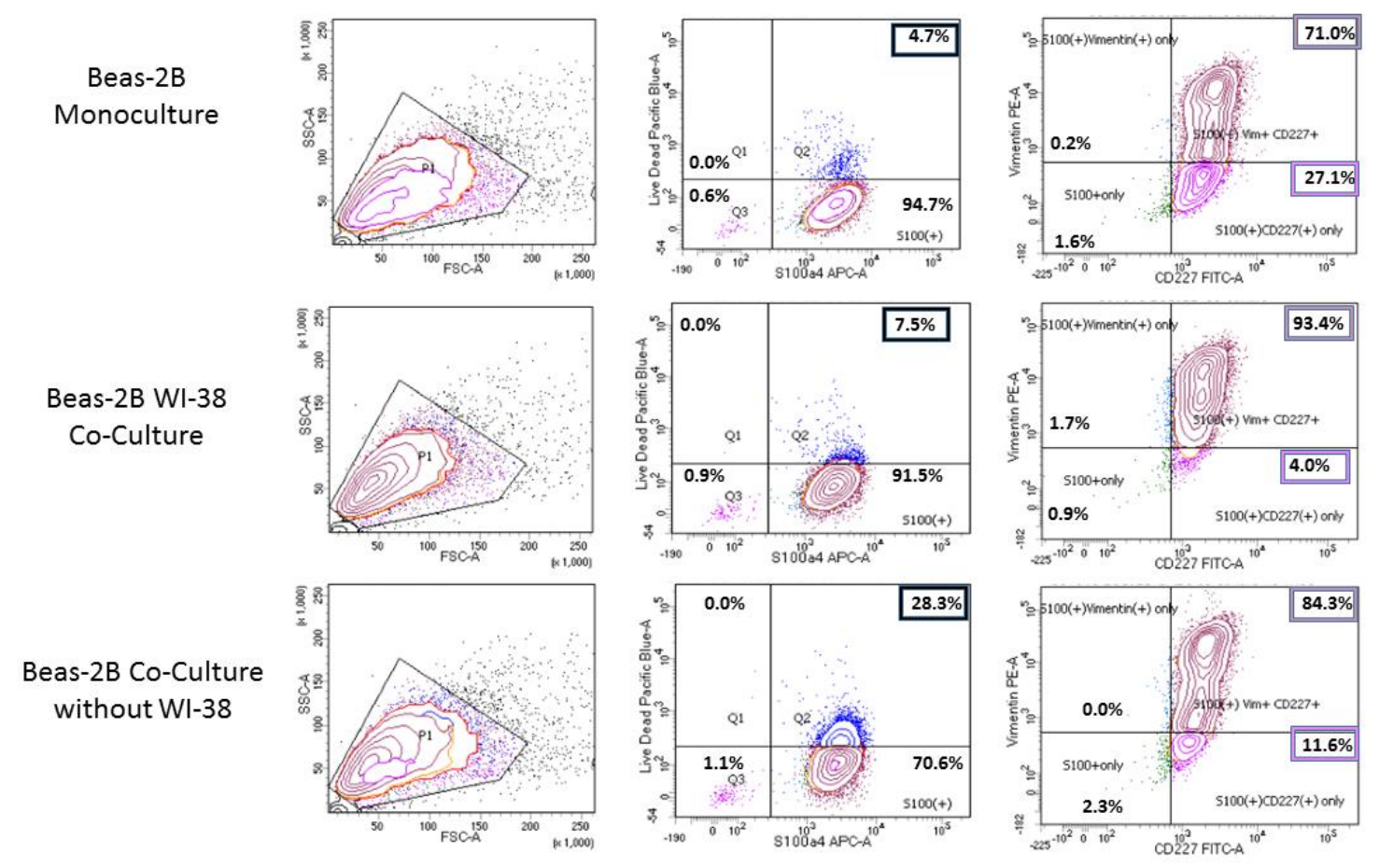

Supplemental Figure 1.7. BEAS-2B in DMEM F-12 alter expression of cell phenotype markers changes

with culture conditions alone. Flow cytometry was used to monitor changes in cell phenotype markers vimentin, S100A4, and CD-227 as well as cell death by live/dead staining in BEAS-2B seeded in monoculture, after 72 hours in co-culture, and after 72 hours alone on Transwells ${ }^{\circledR}$ with DMEM-F12 media only below. Results suggested that some cell death occurred in BEAS-2B alone on Transwells ${ }^{\circledR}$, but co-culture conditions increased the percentage of BEAS-2B co-expressing S100A4, vimentin, and CD227 and decreased the percentage of cells co-expressing S100A4 and CD-227 in co-culture and coculture without WI-38. 

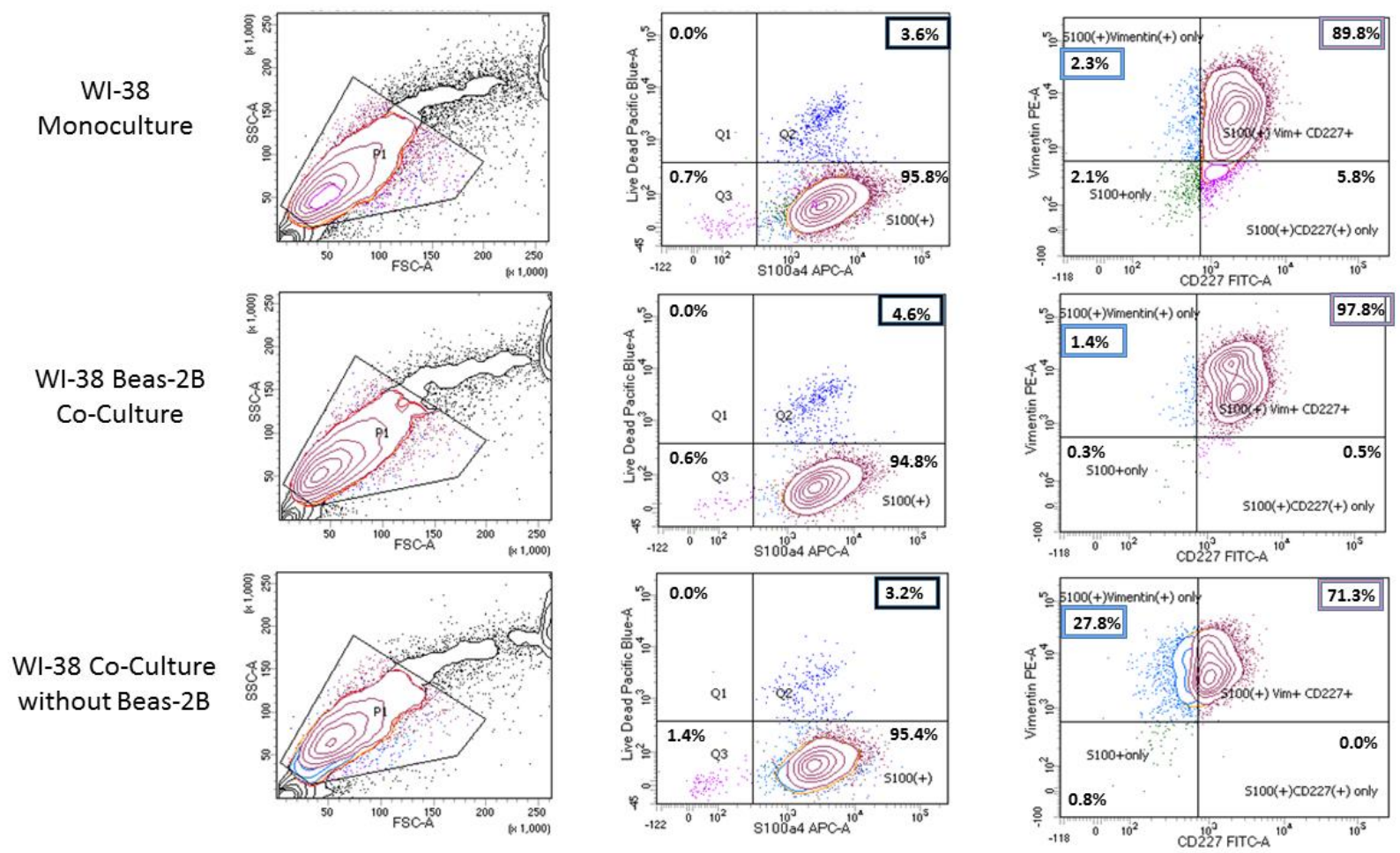

Supplemental Figure 1.8. WI-38 in DMEM F-12 alter expression of cell phenotype markers changes

with culture conditions alone. Flow cytometry was used to monitor changes in cell phenotype markers vimentin, S100A4, and CD-227 as well as cell death by live/dead staining in WI-38 seeded in monoculture, after 72 hours in co-culture, and after 72 hours alone beneath Transwells ${ }^{\circledR}$ with BEAS-2B DMEM-F12 media only above. Results suggested that limited cell death occurred, and the percentage of WI-38 co-expressing S100A4 and vimentin in co-culture without the presence of BEAS-2B increased, and altered co-expression ofS100A4, vimentin, and CD-227 in co-culture and co-culture without the presence of BEAS-2B. 


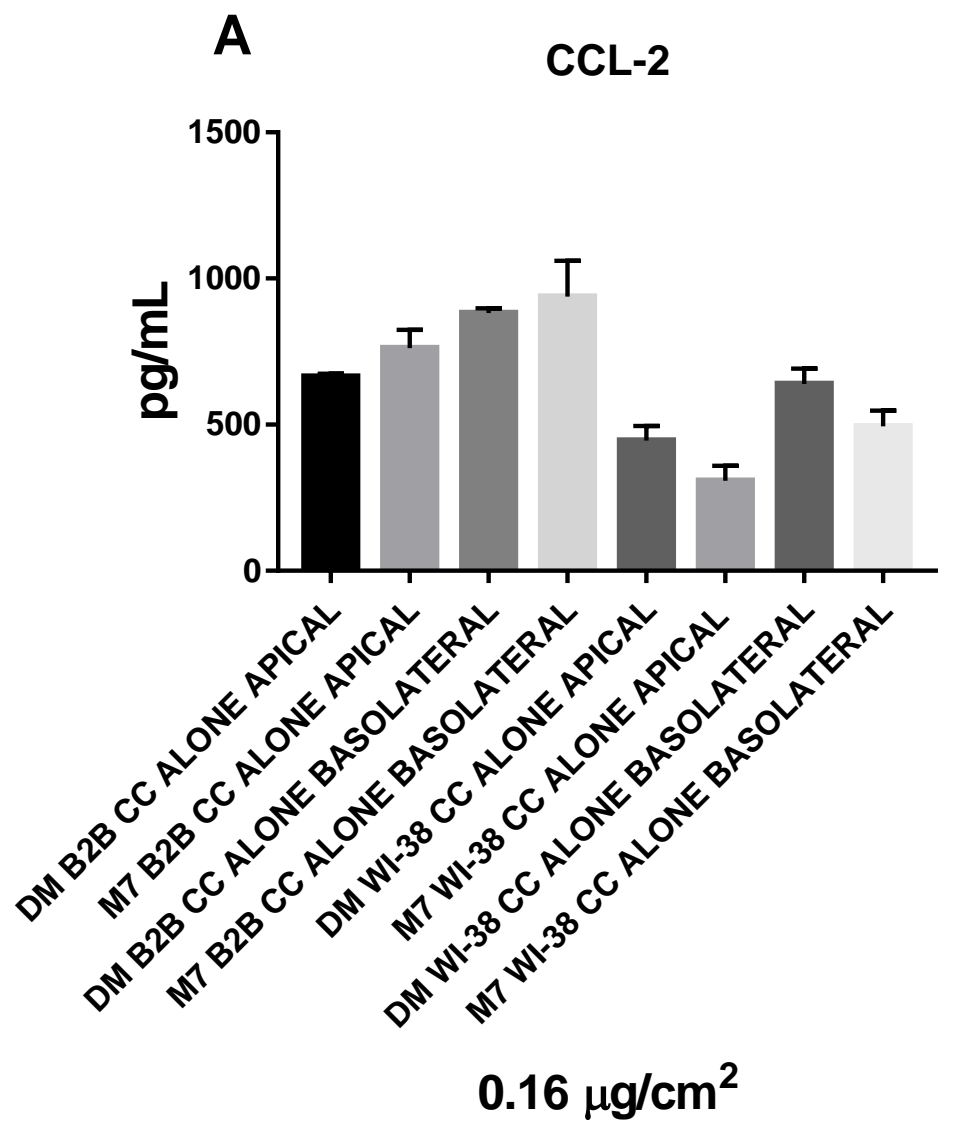

B

CCL-2

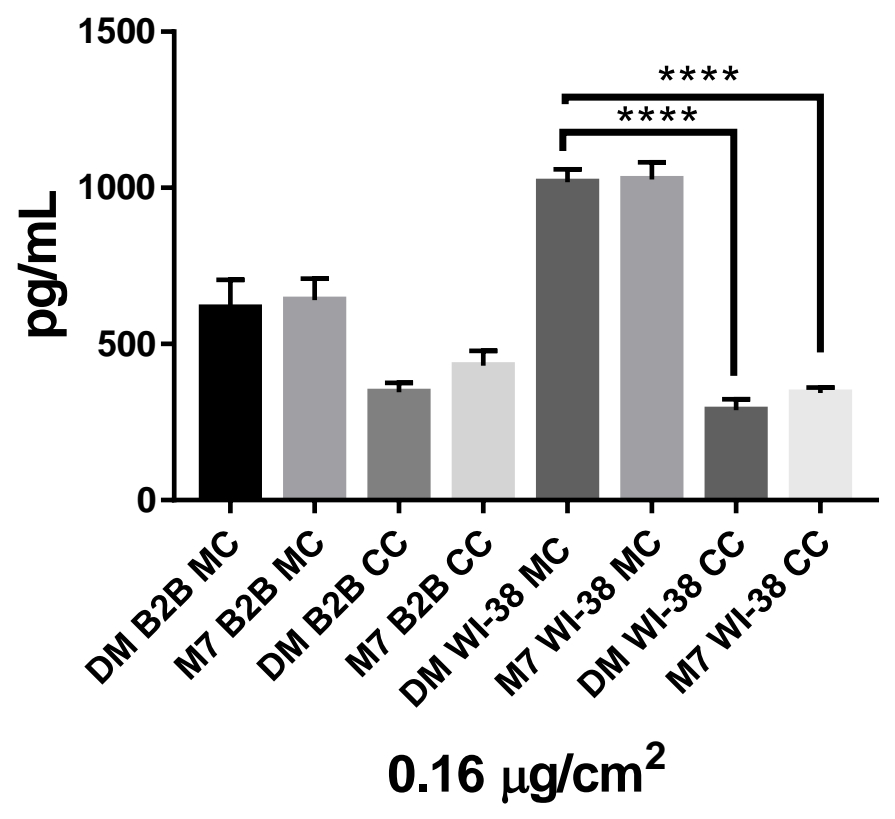




\section{Supplemental Figure 1.9. CCL2 production is altered in BEAS-2B and WI-38 under various culture}

conditions. BEAS-2B and WI-38 were seeded in monoculture, in co-culture, and alone in the apical or basolateral chamber of a Transwell ${ }^{\circledR}$, respectively. Media samples from apical and basolateral wells were collected 24 hours after exposure to $0.16 \mu \mathrm{g} / \mathrm{cm}^{2}$ DM or MWCNT-7, spun down to remove debris and extraneous MWCNT-7, and assayed by ELISA in a pilot experiment of the BEAS-2B-WI-38 co-culture system. Results suggest that there are no significant changes in CCL2 between DM and MWCNT-7 treated samples within the same culture conditions. Results also argued against pursuing further study of CCL2 within the system, especially due to significant decreases in CCL2 production in BEAS-2B and WI38 in co-culture when compared with monoculture in $\mathbf{B}(* * * *, \mathrm{p}<0.0001)$. 


\section{Supplemental Results:}

\subsection{SAEC expression of cell phenotype markers changes with culture conditions alone.}

During the early phases of this project, the author found it important to understand the influence of the Transwell ${ }^{\circledR}$ system and presence of another cell type and cell media on phenotype and viability of the SAEC and WI-38 used without the presence of MWCNT-7. Since WI-38 were found to not be amenable to growth on coverslips for confocal microscopy and we did not want to coat the coverslips with an extracellular matrix protein that could alter WI-38 phenotypic behavior, we decided to use flow cytometry for a preliminary experiment. Three epithelial and fibroblast markers including CD-227 (epithelial), vimentin (fibroblast), and S100A4 (also known as Fibroblast Specific Protein 1) were assessed to monitor changes in cell phenotype over the course of experimentation. LIVE/DEAD labeling was also carried out to determine if culture conditions altered cell viability. SAEC samples were collected under "pre co-culture" conditions, meaning that the SAEC were seeded on Transwells ${ }^{\circledR}$ but not exposed to WI-38 or EMEM media in the basolateral chamber, co-culture conditions with WI-38 in the basolateral chamber, and alone on Transwells $^{\circledR}$ with only WI-38 EMEM media in the basolateral chamber. 11.8 percent SAEC died under pre co-culture conditions, 12.6 percent under co-culture conditions, and 13.7 percent in co-culture without WI-38 (Supplemental Figure 1.1). Nearly all cells assessed were double-positive for CD-227 and vimentin or S100A4, suggesting that our fibroblast markers were not just specific for fibroblasts. Despite this, vimentin-only expressing SAEC increased to 7.2 percent under co-culture conditions concomitant with an increase in S100A4-only expressing to 6.9 percent, which was resolved in the absence of WI-38 (even if EMEM media was present). Together, these data suggested that culture conditions alone could potentially affect cell behavior and viability.

\subsection{WI-38 expression of cell phenotype markers changes with culture conditions alone}

Similar to studies in SAEC, WI-38 were assessed by flow cytometry to monitor changes in cell phenotype using CD-227, Vimentin, and S100A4. Nearly all WI-38 were double positive for CD-227 and vimentin or 
S100A4, and less than 2 percent were labeled with vimentin or S100A4 only. 17.2 percent WI-38 died under pre co-culture conditions, 14.1 percent under co-culture conditions, and 7.7 percent in co-culture without SAEC (Supplemental Figure 1.2.).

\subsection{CCL2 production is altered in SAEC and WI-38 exposed to $1.2 \mu \mathrm{g} / \mathrm{mL} M W C N T-7$ under various culture conditions.}

CCL2, a CC-chemokine that is a potent chemotactic factor for macrophage to sites of injury, is produced by a variety of cell types including epithelial, endothelial, fibroblast, and monocytic cells [199]. CCL2 was elevated in BAL fluid and fibrotic lung tissues of patients with idiopathic pulmonary fibrosis, which was also corroborated by mouse studies $[200,201]$. In vivo studies carried out in CCR2-/- mice showed decreases in macrophage infiltration and MMP activity; bleomycin-induced fibrosis was also attenuated by overexpression of a CCL2 dominant negative inhibitor [202, 203]. Pulmonary epithelium was identified as a primary source of CCL2 in fibrotic lungs, and previous in vitro results obtained in our lab suggested that SAEC released CCL2 after $24 \mathrm{~h}$ exposure to $1.2 \mu \mathrm{g} / \mathrm{mL}$ MWCNT-7 [91, 201]. Based upon this evidence, we believed that CCL2 could play a significant role in a co-culture system of SAEC and WI38 fibroblasts as part of a relatively novel mechanism of action of MWCNT-7 induced fibrosis. The experiment was designed to assess CCL2 production of SAEC or WI-38 in monoculture, co-culture, SAEC in the apical chamber of a Transwell ${ }^{\circledR}$, and WI-38 alone in basolateral chamber of a Transwell ${ }^{\circledR}$ to: 1) compare between culture conditions, 2) determine if CCL2 passed through the Transwell ${ }^{\circledR}$ membrane, and 3) determine if MWCNT-7 exposure significantly altered CCL2 production. Each culture system was exposed to $1.2 \mu \mathrm{g} / \mathrm{mL}$ DM or MWCNT-7 in the apical well for $24 \mathrm{~h}$, and conditioned media was collected from the apical and basolateral chambers of each condition (monoculture samples were collected from a typical 6 well dish). Expression levels of CCL2 are presented for apical and basolateral wells of SAEC and WI-38 alone in Supplemental Figure 1.3. A and SAEC and WI-38 in monoculture and co-culture in Supplemental Figure 1.3.B. Results suggest that when grown alone, SAEC produce CCL2 that can pass 
through the Transwell ${ }^{\circledR}$ membrane into the basolateral chamber, however with significantly less CCL2 present compared to the apical SAEC control well. As expected, WI-38 seeded alone in the basolateral chamber showed limited presence of CCL2 in conditioned media collected from the apical or basolateral chambers, which suggested that the presence of SAEC media or MWCNT-7 above WI-38 had limited effects on CCL2 production (Supplemental Figure 1.3. A). In Supplemental Figure 1.3. B, monoculture and co-culture conditions were compared for SAEC and WI-38. No significant difference in CCL2 production was noted between monoculture and co-culture conditions of SAEC. However, CCL2 levels were significantly increased in WI-38 co-culture samples compared to WI-38 monoculture. We believe the majority of the CCL2 in WI-38 co-culture samples came from SAEC. For all samples assayed, there was no difference between DM and MWCNT-7 exposed samples as well.

\subsection{Changes in dose between monoculture and co-culture samples yield no significant changes in CCL2 production in SAEC and WI-38 within exposure groups.}

An additional study to determine the impact of surface area based dosimetry between monoculture and co-culture exposures on CCL2 was carried out. Since the surface area of a Transwell ${ }^{\circledR}$ is $4.67 \mathrm{~cm}^{2}$ and a six well dish is $9.5 \mathrm{~cm}^{2}$, exposing cells based upon concentration alone would in essence deliver a greater dose of MWCNT-7 to Transwells ${ }^{\circledR}$ compared to monoculture experiments, and therefore make the comparison of monoculture to co-culture more challenging. To be in accord with Porter's mouse study, an $80 \mu \mathrm{g} /$ mouse dose was converted into $0.16 \mu \mathrm{g} / \mathrm{cm}^{2}$ in vitro dose that could be normalized between the Transwell ${ }^{\circledR}$ and six well dish exposures. The goals of this experiment were similar to those above, namely: 1) compare between culture conditions, 2) determine if CCL2 passed through the Transwell ${ }^{\circledR}$

membrane, and 3) determine if MWCNT-7 exposure significantly altered CCL2 production. Results were quite similar to those observed in the experiments where all exposures were carried out at $1.2 \mathrm{\mu g} / \mathrm{mL}$ (which exposed cells to $0.38 \mu \mathrm{g} / \mathrm{cm}^{2}$ and $0.19 \mu \mathrm{g} / \mathrm{cm}^{2}$ in Transwells ${ }^{\circledR}$ and monoculture, respectively) 
(Supplemental Figure 1.4. A). Again, no significant differences were observed between DM and MWCNT-7 exposed samples within the same culture condition (Supplemental Figure 1.4. A, B).

\subsection{BEAS-2B expression of cell phenotype markers changes with culture conditions in a system using DMEM F-12 and EMEM media.}

Due to technical difficulties with maintaining consistent SAEC and WI-38 cell viability in co-culture, BEAS2B human bronchial epithelial cells were selected as a potential replacement. BEAS-2B have been used in a wide variety of pulmonary disease studies and are more hardy than SAEC, providing a logical choice for additional attempts at developing a co-culture system. Three markers including CD-227, vimentin, and S100A4 were selected to monitor changes in cell phenotype over the course of experimentation. LIVE/DEAD labeling was also carried out to determine if culture conditions altered cell viability. BEAS-2B samples were collected under "pre co-culture" conditions, such that BEAS-2B were seeded on Transwells ${ }^{\circledR}$ in DMEM F-12 but not exposed to WI-38 or EMEM media, co-culture conditions with WI-38 in the basolateral chamber, and on Transwells ${ }^{\circledR}$ with only EMEM media in the basolateral chamber. Results suggested that growth on $\operatorname{Transwells}^{\circledR}$, even before the introduction of fibroblasts or their media, altered BEAS-2B marker expression. Nearly all BEAS-2B were S100A4 positive, and cells were gated off of this population. In pre co-culture BEAS-2B, 79.2 percent of cells co-expressed S100A4 and vimentin, while 20.7 percent co-expressed S100A4, vimentin, and CD-227. Conversely, 30.8 percent of BEAS-2B in co-culture exhibited co-expression of S100A4 and vimentin, and 69.0 percent co-expressed S100A4, vimentin, and CD-227. BEAS-2B grown on Transwells ${ }^{\circledR}$ in the presence of EMEM only in the basolateral chamber exhibited labeling similar to that of pre co-culture condition, with 68.7 percent coexpressing S100A4 and vimentin and 31.2 percent co-expressing S100A4, vimentin, and CD-227

(Supplemental Figure 1.5.). These data suggested that a significant shift in cell phenotype occurred in the co-culture condition compared to others, and that the shift could potentially be of important consideration when analyzing results. 


\subsection{WI-38 expression of cell phenotype markers changes with culture conditions in a system using}

DMEM F-12 and EMEM media.

In order to address changes in WI-38 marker expression from a co-culture system of BEAS-2B and WI-38 using DMEM F-12 and EMEM medias, respectively, CD-227, vimentin, and S100A4 were analyzed. WI-38 samples were collected under "pre co-culture" conditions, such that WI-38 were seeded in the basolateral chamber of a Transwell ${ }^{\circledR}$ in EMEM only, co-culture conditions, and in the basolateral chamber of Transwells ${ }^{\circledR}$ with DMEM F-12 media only in the apical chamber. Results suggested that coculture conditions with or without the presence of BEAS-2B altered marker expression in WI-38. WI-38 were gated off of the S100A4 positive population for further analysis. In pre co-culture WI-38, 18.6 percent of cells co-expressed S100A4 and vimentin, while 81 percent co-expressed S100A4, vimentin, and CD-227. Conversely, $\mathrm{WI}-38$ in co-culture were 49.6 percent co-expressed S100A4 and vimentin and 50.0 percent co-expressed S100A4, vimentin, and CD-227. WI-38 grown in the basolateral chamber of Transwells $^{\circledast}$ in the presence of DMEM F-12 only in the apical chamber exhibited labeling similar to an almost intermediary phenotype between pre co-culture and co-culture, with 40.4 percent co-expressing S100A4 and vimentin and 59.0 percent coexpressing S100A4, vimentin, and CD-227 (Supplemental Figure 1.5.). These data suggested that a significant shift in cell phenotype occurred in the co-culture conditions compared to pre co-culture, and that the shift could potentially be of important consideration when analyzing results.

\subsection{BEAS-2B expression of cell phenotype markers changes with culture conditions alone in the presence of DMEM F-12 only.}

Another iteration of the BEAS-2B:WI-38 co-culture system was assessed for cell phenotype markers CD227, vimentin, and S100A4. In these experiments, BEAS-2B and WI-38 were only grown in DMEM F-12 media. In the absence of EMEM, BEAS-2B phenotypes were quite changed from the experiment shown in Supplemental Figure 1.4. In this preliminary experiment, a monoculture control in normal six well 
dishes were used in favor of the pre co-culture control in order to compare between cells that should be comfortably growing on their typical cell culture substrate. BEAS-2B were gated off of the S100A4 positive population for further analysis. In monoculture BEAS-2B, 27.1 percent of cells co-expressed S100A4 and CD-227, while 71.0 percent co-expressed S100A4, Vimentin, and CD-227. 4.0 percent of BEAS-2B in co-culture co-expressed S100A4 and CD-227 and 93.4 percent co-expressed S100A4, vimentin, and CD-227. BEAS-2B grown in the apical chamber of Transwells ${ }^{\circledR}$ in the presence of DMEM F12 only in the basolateral chamber exhibited labeling similar to an almost intermediary phenotype between monoculture and co-culture, with 11.6 percent co-expressing S100A4 and CD-227 and 84.3 percent co-expressing S100A4, vimentin, and CD-227 (Supplemental Figure 1.7.). These results suggested that the selection of media for use in a co-culture system could have a large impact on the cell phenotypes and marker expression of the cell lines we chose.

1.8 WI-38 expression of cell phenotype markers changes with culture conditions alone in the presence of DMEM F-12 alone.

As the companion experiment shown in Supplemental Figure 1.7., WI-38 were assessed in the same experiment for marker expression of CD-227, vimentin, and S100A4 in the presence of DMEM F-12 media. WI-38 were gated off of the S100A4 positive population for further analysis. In monoculture WI38, 2.3 percent of cells co-expressed S100A4 and vimentin, 5.8 percent co-expressed S100A4 and CD227, and 89.8 percent co-expressed S100A4, vimentin, and CD-227. 1.4 percent of WI-38 in co-culture co-expressed S100A4 and vimentin, 0.5 percent co-expressed S100A4 and CD-227, and 97.8 percent coexpressed S100A4, vimentin, and CD-227. WI-38 grown in the basolateral chamber of Transwells ${ }^{\circledR}$ in the presence of DMEM F-12 only exhibited significant changes, with 27.8 percent coexpressing S100A4 and vimentin, 0.0 percent co-expressing S100A4 and CD-227, and 71.3 co-expressing S100A4, vimentin, and CD-227 (Supplemental Figure 1.8.). These results suggested that the presence of DMEM F-12 as 
opposed to EMEM generally pushed WI-38 towards a triple positive phenotype and reiterated the impact of media selection for use in co-culture systems.

\subsection{CCL2 production is altered in BEAS-2B and WI-38 under various culture conditions in the presence of DMEM F-12 only.}

In an effort to develop an epithelial-fibroblast co-culture system despite issues with SAEC, BEAS-2B bronchial epithelial cells were studied as a potential replacement. As previously described, BEAS-2B and WI-38 were seeded in monoculture, co-culture, BEAS-2B alone in the apical chamber of a Transwell ${ }^{\circledR}$, and WI-38 alone in the basolateral chamber of a Transwell ${ }^{\circledR}$. All cell types were monitored in DMEM F12. Each culture system was exposed to $0.16 \mu \mathrm{g} / \mathrm{cm}^{2}$ DM or MWCNT-7 in the apical well for $24 \mathrm{~h}$, and conditioned media was collected from the apical and basolateral chambers of each condition (monoculture samples were collected from a typical 6 well dish). No significant changes in CCL2 levels were observed in the apical or basolateral wells of BEAS-2B cultured alone. However, CCL2 trended higher in basolateral media of BEAS-2B alone, suggesting that a larger concentration of CCL2 distributed into the basolateral chamber. Additionally, CCL2 protein levels were higher in the apical and basolateral chambers of WI-38 alone, suggesting that perhaps the DMEM F-12 media used in the experiments spurred some degree of WI-38 activity compared to SABM (Supplemental Figure 1.9. A). No significant changes were observed between DM and MWCNT-7 exposures per culture condition. Monoculture and co-culture conditions were also assessed for CCL2 production, and unlike results obtained with SAEC, media collected from BEAS-2B in co-culture had decreased CCL2 production (Supplemental Figure 1.9. B). Differences between WI-38 monoculture and co-culture conditions suggested the exact opposite of SAEC results; CCL2 production in WI-38 co-culture samples was significantly diminished compared to monoculture. Together, these results led us to believe that perhaps studying CCL2 mediated pulmonary fibrogenic signaling was unlikely in the co-culture system, and that pervasive, recurring issues with 
cellular viability and phenotype issues within the co-culture systems attempted would suggest utilizing a different method of study.

\section{Supplemental Methods:}

\subsection{Cell Culture}

SAEC were a gift from Tom K. Hei (Columbia University, New York, NY) (Piao et al. 2005). SAEC were cultured in serum-free SABM medium supplemented with a SAGM SingleQuot kit of growth factors, cytokines, and supplements (Lonza Walkersville, Inc.; Walkersville, MD). BEAS-2B bronchial epithelial cells were purchased from ATCC and maintained in DMEM: F-12 (ATCC, Manassas, VA) with 10\% FBS (Atlanta Biologicals, Norcross, GA) and 5\% percent penicillin-streptomycin (Lonza, Walkersville, MD), and all experiments were carried out using cells below passage 15 . WI-38 normal human lung fibroblasts were purchased from ATCC and maintained in EMEM (ATCC) with 10\% FBS (Atlanta Biologicals) and 5\% penicillin-streptomycin (Lonza), and all experiments were carried out using cells below passage 9. All cell types were maintained in an incubator at $37^{\circ} \mathrm{C}$ with $5 \% \mathrm{CO}_{2}$.

\subsection{Co-Culture Attempts and Methodology}

Numerous attempts were made to develop an epithelial-fibroblast co-culture system that would be functional. Preliminary experiments suggested that significant cell death occurred if SAEC or WI-38 were grown in each other's respective medias, SABM or EMEM, in monoculture. SAEC were also not amenable to growth in alternative media such as DMEM which may have suited the WI-38 in short-term exposures. The earliest iteration of the project attempted to seed SAEC on one side of a Millicell $0.4 \mu \mathrm{m}$ PET (polyethylene terephthalate) Insert (EMD Millipore, Billerica, MA) with WI-38 on the other side of the membrane. WI-38 were not amenable to growth on the underside of the insert, even after coating with manufacturer's suggested concentrations of VitroCol (Advanced Biomatrix, San Diego, CA) or fibronectin

(BD Bioscience). This idea was abandoned in favor of using PET Transwells ${ }^{\circledR}$ with $0.4 \mu \mathrm{m}$ pore size (Corning, Corning, NY) previously used in the lab [19]. To prepare a co-culture plate, the Transwell ${ }^{\circledR}$ inserts 
were hydrated in SABM with serum for an hour, and 125,000 SAEC were seeded on Transwells ${ }^{\circledR}$. For monoculture controls for ELISA assays, 200,000 SAEC were seeded in six well dishes. 40,000 WI-38 would be seeded in companion six well dishes. Media would be changed on both cell types the next day, and Transwells ${ }^{\circledR}$ would be transferred to WI-38-seeded dishes on Day 3. If cells were to be treated, they were exposed to DM or MWCNT-7 at $1.2 \mu \mathrm{g} / \mathrm{mL}$, or later, $0.16 \mu \mathrm{g} / \mathrm{cm}^{2}$ on Day 4, and endpoints were assessed $24 \mathrm{~h}$ on Day 5. The system was extremely demanding to utilize and maintain consistently; unexplained cell death remained an issue over the several months we attempted using the system. Since we knew the SAEC were a relatively delicate cell type, we opted to move forward into using BEAS-2B as an alternative. BEAS-2B are hardier, SV-40 transformed human bronchial epithelial cells that have been grown in a wide variety of medias by others including DMEM F-12, RPMI 1640, BEGM, KGM, LHC-9 and others, which suggested that perhaps these would be hardy enough to withstand co-culture treatment [204-207]. As we had great difficulty with SAEC in co-culture, we developed a 4 day experimental design in which BEAS2B and WI-38 would be seeded the same day. WI-38 would be seeded at 100,000 cells first and allowed to seed 6 hours in EMEM. BEAS-2B would be seeded at 100,000 cells on pre-wet Transwell ${ }^{\circledR}$ membranes placed on top of WI-38. Cells would also be seeded in monoculture at 150,000 per well for controls. The co-culture system would become more established over the next day, and BEAS-2B would be exposed to $0.16 \mu \mathrm{g} / \mathrm{cm}^{2}$ DM or MWCNT-7 Day 3. Endpoints would be assessed $24 \mathrm{~h}$ later on Day 4. Additional earlier preliminary experimentation was carried out in the BEAS-2B-WI-38 system to determine if various methodologies could improve the system. BEAS-2B and WI-38 co-cultures were set up as follows: Beas2B and WI-38 seeded together on Day 1 in DMEM F-12 and EMEM, respectively; BEAS-2B and WI-38 seeded together on Day 1 in DMEM F-12 and DMEM F-12, respectively (results shown in Supplemental Figure 1,7 and 1.8.); BEAS-2B and WI-38 seeded separately on Day 1 in DMEM F-12 and EMEM, respectively, and brought together on Day 2 when all media was changed to DMEM F-12; and WI-38 seeded on Day 1 in EMEM, brought together with BEAS-2B seeded on Day 2 in DMEM F-12. Upon visual 
inspection, the cells appeared to exhibit morphology most similar to monoculture when both cell types were in DMEM F-12, which was used for CCL2 ELISAs.

\section{3.. Flow Cytometry Assessment of Cell Markers}

Several preliminary experiments were carried out utilizing flow cytometry to assess various cell markers including epithelial markers CD-326 (EpCAM) and CD-227 (MUC1) (Affymetrix, Santa Clara, CA), and fibroblast markers vimentin (Cell Signaling Technology, Danvers, MA), and S100A4 (also known as FSP-1) (Abcam, Cambridge, UK). The CD-326 antibody, despite carrying out a titration experiment, did not respond as expected and was removed from analysis. Briefly, SAEC were seeded on Transwells ${ }^{\circledR}$ for three separate purposes to observe "pre co-culture" conditions prior to the addition of WI-38 with SABM media in and under the Transwells ${ }^{\circledR}$, co-culture conditions with the addition of WI-38 on Day 3 of the experiment, and alone on Transwells ${ }^{\circledR}$ with the addition of WI-38 EMEM media only in the basolateral chamber on Day 3. WI-38 were seeded in the bottom of Transwells ${ }^{\circledR}$ plates on Day 1, and allowed to seed prior to introduction of the Transwells ${ }^{\circledR}$ back into the system on Day 3 during which the SAEC would also be serum starved. Samples were collected on Day 5 of experimentation. First, all samples were subjected to labeling with LIVE/DEAD fixable dead stain per manufacturer's protocol, then a BD Cytofix/Cytoperm Fixation/Permeabilization Kit (Becton Dickinson, Franklin Lakes, NJ) was utilized per manufacturer's protocol to prepare cells for fixation and labeling with CD-227, vimentin, and S100A4. After labeling, cells were stored in PBS $+1 \% \mathrm{BSA}$ at $4^{\circ} \mathrm{C}$ until assessment by flow cytometry utilizing a BD LSRFortessa (Becton Dickinson). Cells were gated using forward scatter and side scatter, and only live cells were gated for analysis of CD-227, vimentin, and S100A4. Dot plots provide percentages of each marker assessed under all three culture conditions.

Due to unpredictable technical difficulties with SAEC, BEAS-2B were also utilized in a similar experiment. BEAS-2B were seeded on Transwells ${ }^{\circledR}$ for three separate purposes to observe "pre co-culture" conditions prior to addition of WI-38, co-culture conditions with the addition of WI-38, and alone on 
Transwells ${ }^{\circledR}$ with the addition of WI-38 EMEM media only below them. WI-38 were seeded on Day 1 in the basolateral chamber of Transwells ${ }^{\circledR}$ dishes similar to above, and allowed to seed overnight prior to introduction of the Transwells ${ }^{\circledR}$ back into the systems. Samples were collected on Day 4 of experimentation. First, all samples were subjected to labeling with LIVE/DEAD fixable dead stain per manufacturer's protocol, then a BD Cytofix/Cytoperm Fixation/Permeabilization Kit (Becton Dickinson, Franklin Lakes, NJ) was utilized per manufacturer's protocol to prepare cells for fixation and labeling with CD-227, Vimentin, and S100A4. After labeling, cells were stored in PBS $+1 \% \mathrm{BSA}$ at $4^{\circ} \mathrm{C}$ until assessment by flow cytometry utilizing a BD LSRFortessa (Becton Dickinson). Cells were gated using forward scatter and side scatter, and only live cells were gated for further analyses. Cells were further gated for S100A4+ cells, and results are shown in contour plots assessing of S100A4+/CD-227+, S100A4+/Vimentin+, and S100A4+/Vimentin+/CD-227+ cell groups. BEAS-2B and WI-38 were assessed the as described above again, but with WI-38 maintained and treated with DMEM F-12 to better understand the impact of EMEM versus DMEM F-12 on BEAS-2B. The pre co-culture samples were also replaced by a simple monoculture control on plastic dishes for each cell type (as opposed to the Transwells ${ }^{\circledast}$ ) to check if the Transwell ${ }^{\circledast}$ itself was affecting BEAS-2B expression of surface markers.

\subsection{Enzyme Linked Immunosorbent Assay}

Media was collected from SAEC, BEAS-2B, or WI-38 under various growing conditions described above, centrifuged at max speed, and assayed for C-C motif chemokine 2 (CCL2) Conditioned media from each biological replicate were collected and assayed in triplicate for C-C motif chemokine 2 (CCL2) using DuoSet ELISA Development Systems (R\&D Systems, Minneapolis, MN) according to manufacturer's protocol. 


\subsection{Statistical Analysis}

Statistical analyses for the data were performed using GraphPad Prism 7.00. One Way ANOVA tests comparing across all means for sample groups were utilized, and Tukey's Multiple Comparison's Test were used to determine significance between treatment groups, unless otherwise noted. 


\section{Appendix II}

Supplemental Figures, Results, and Methods 


\section{Supplemental Figures:}

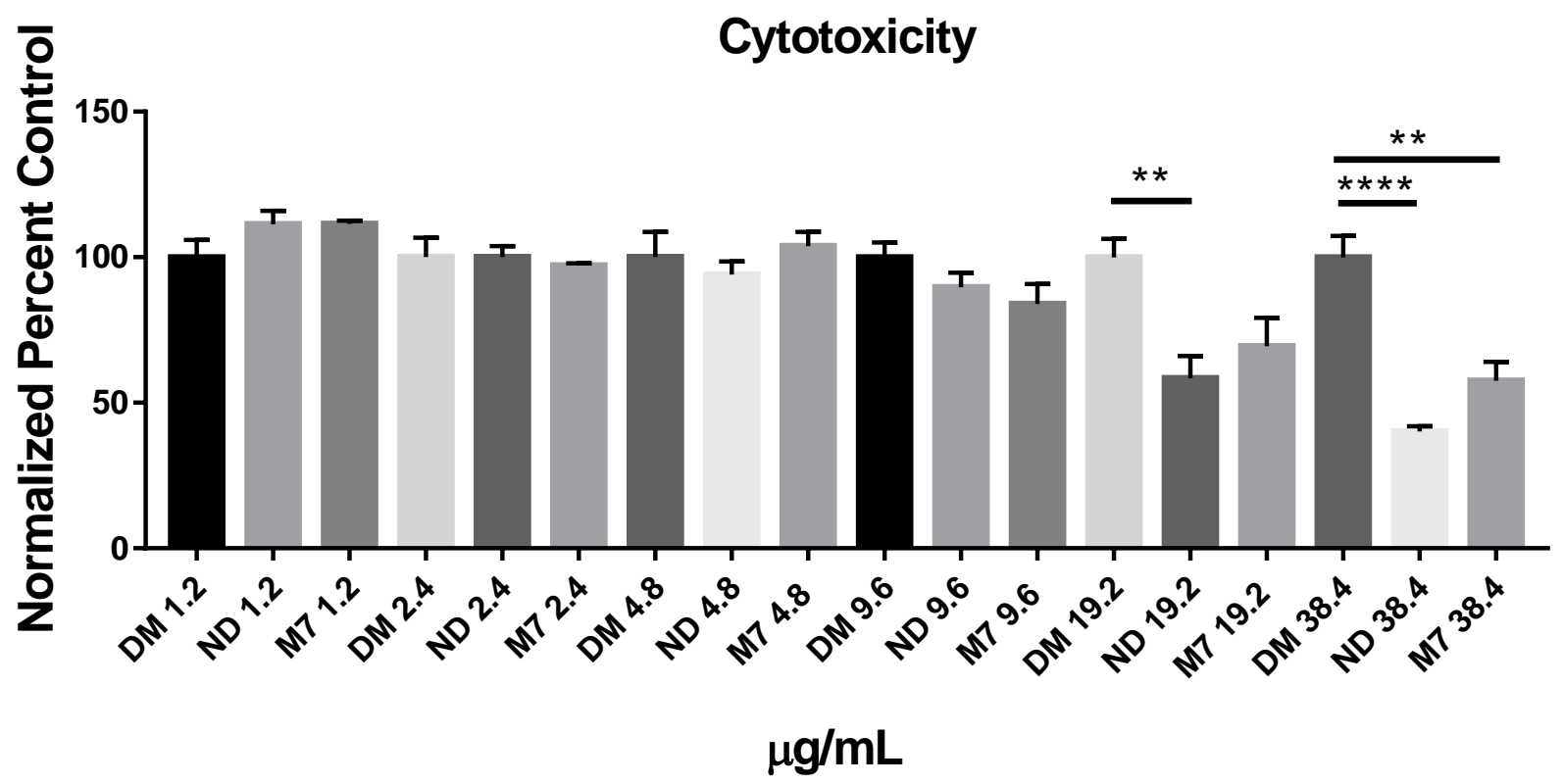

Supplemental Figure 2.1. BEAS-2B proliferation is altered at high exposures to ND-MWCNT and MWCNT-7. BEAS-2B were treated with varying concentrations of DM, ND-MWCNT, or MWCNT-7 for a $24 \mathrm{~h}$ period in three independent experiments. CellTiter $96^{\circledR}$ Aqueous One Solution was added 2 hours prior to end of exposure and absorbance was measured at 490nm. Results indicate that ND-MWCNT and MWCNT-7 decreased BEAS-2B proliferation at 19.2 and $38.4 \mu \mathrm{g} / \mathrm{mL}$ as assessed by One-Way ANOVA and Tukey's multiple comparison test statistical analysis $(* *, p<0.01, * * * *, p<0.0001)$. 


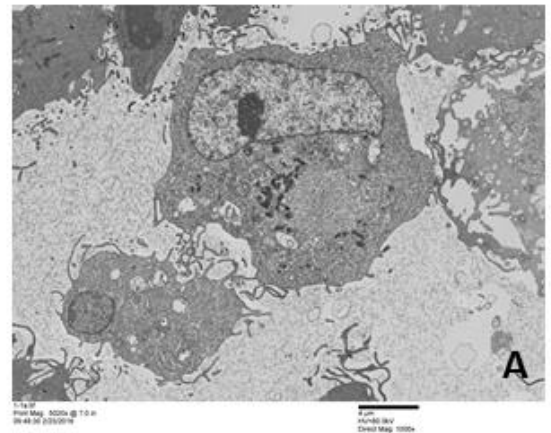

DM
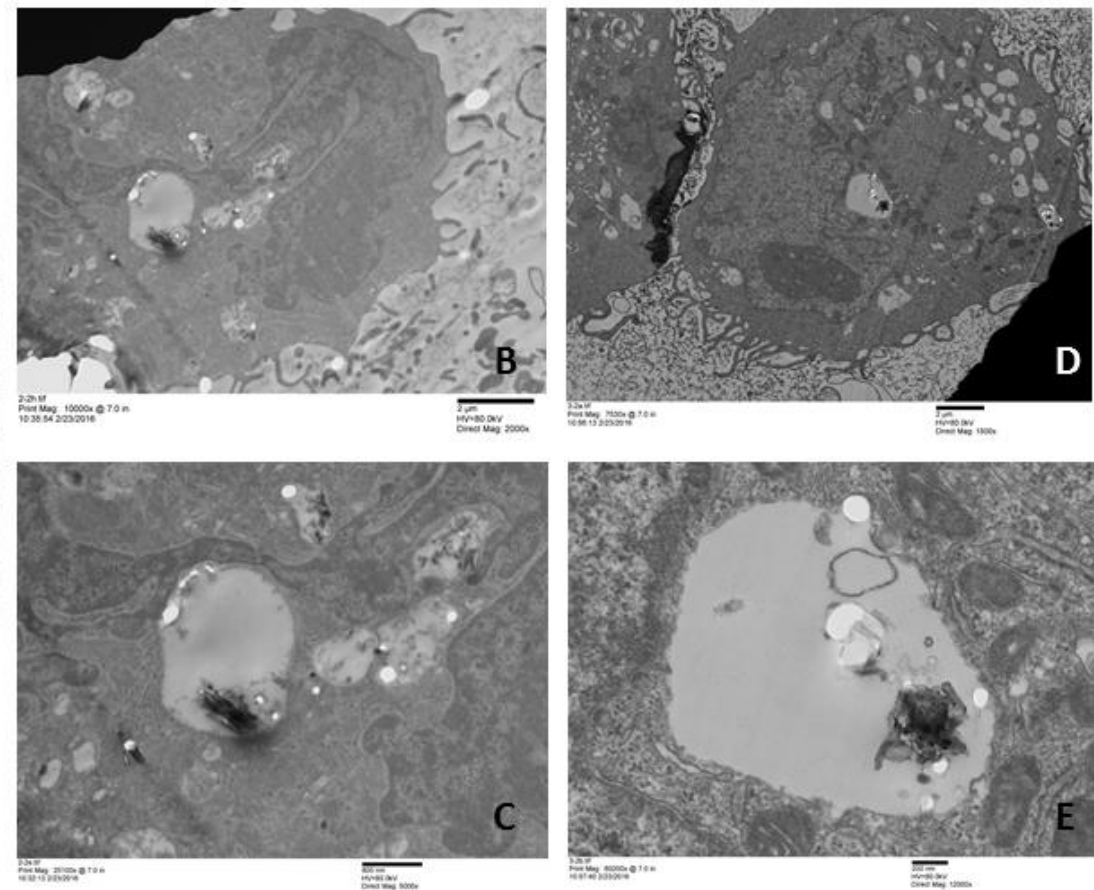

ND

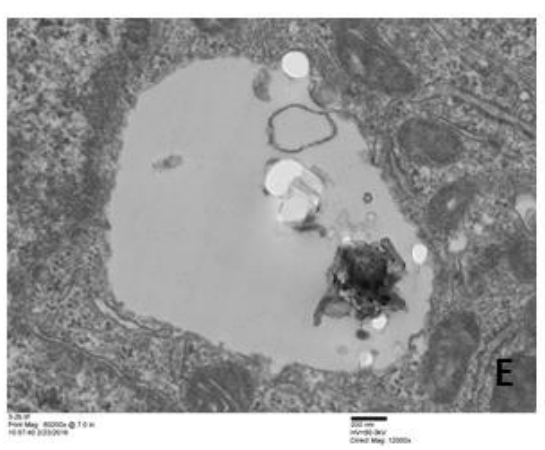

M7

\section{$4.8 \mu \mathrm{g} / \mathrm{mL}$}

Supplemental Figure 2.2. BEAS-2B engulf ND-MWCNT or MWCNT-7. TEM analysis of BEAS-2B exposed to $4.8 \mu \mathrm{g} / \mathrm{mL}$ ND-MWCNT or MWCNT-7 over 24 hours. 


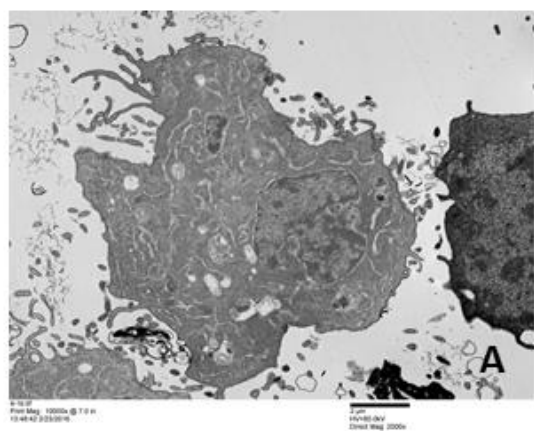

DM
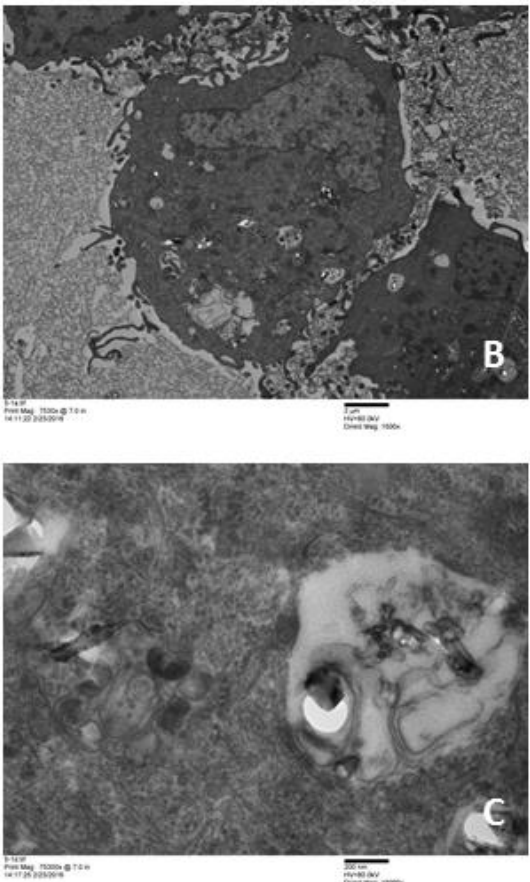

ND
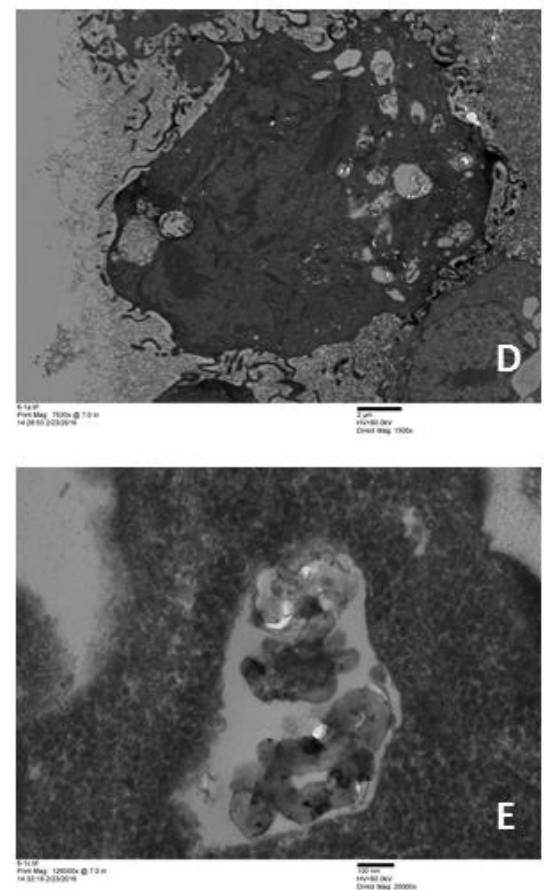

M7

$9.6 \mu \mathrm{g} / \mathrm{mL}$

Supplemental Figure 2.3. BEAS-2B engulf ND-MWCNT or MWCNT-7. TEM analysis of BEAS-2B exposed to $9.6 \mu \mathrm{g} / \mathrm{mL}$ ND-MWCNT or MWCNT-7 over 24 hours. 
4.8 or $9.6 \mu \mathrm{g} / \mathrm{mL}$ NDMWCNT or MWCNT-7

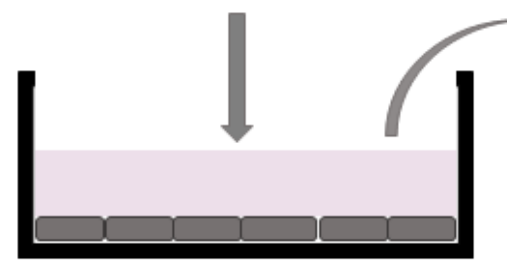

Beas-2B
Treat WI-38 48h with conditioned media

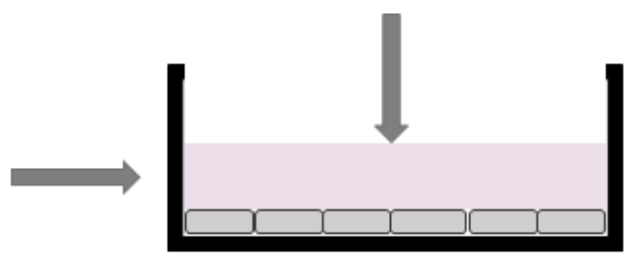

WI-38

Supplemental Figure 2.4. Experimental design for treating WI-38 with conditioned media. A

conditioned media model using BEAS-2B and WI-38 was used to assess preliminary endpoints. BEAS-2B were treated with 4.8 or $9.6 \mu \mathrm{g} / \mathrm{mL}$ of DM, ND-MWCNT, or MWCNT-7 for $24 \mathrm{~h}$. Conditioned media was collected, spun down, and added to WI-38 for $48 \mathrm{~h}$ prior to further assessment. 


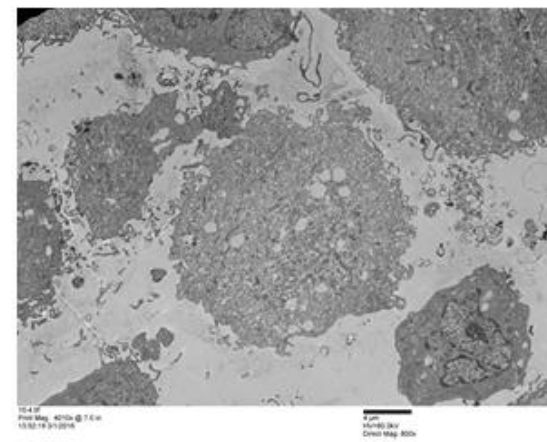

DM

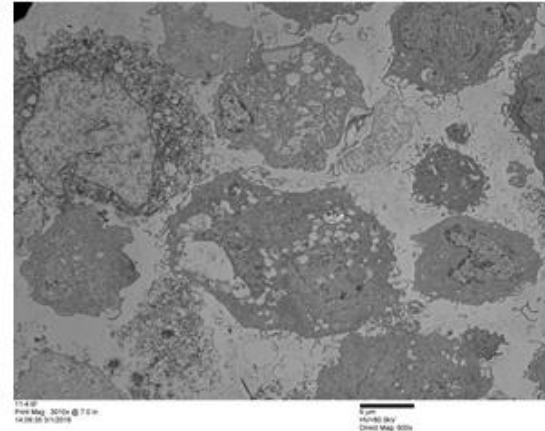

ND

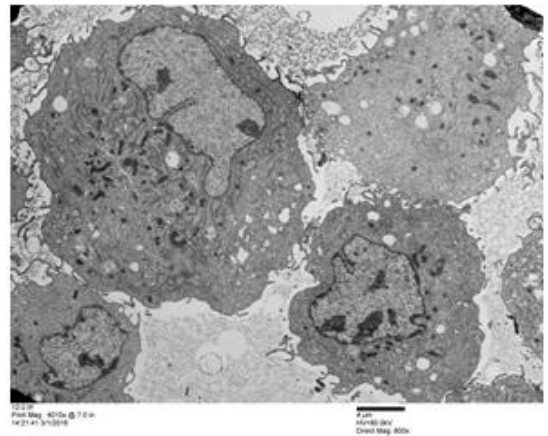

M7

\section{$4.8 \mu \mathrm{g} / \mathrm{mL}$}

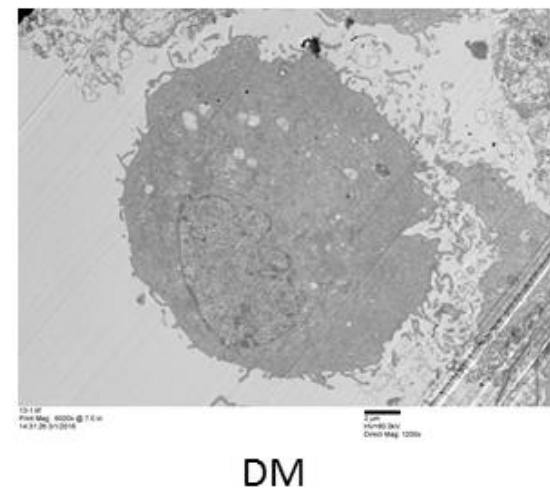

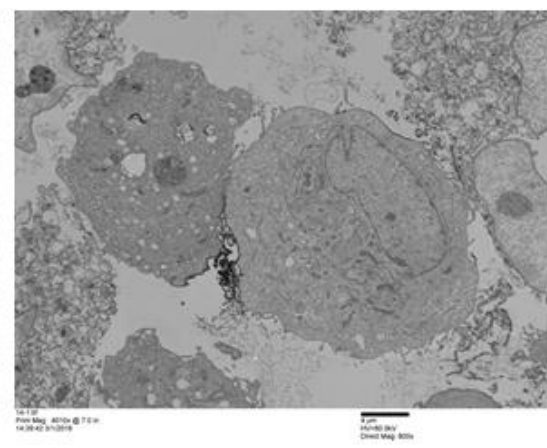

ND

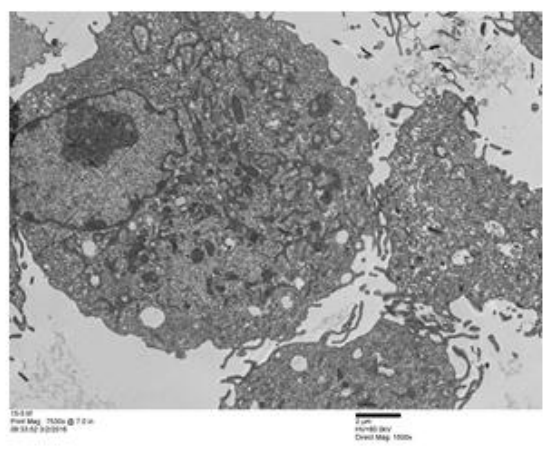

M7

\section{$9.6 \mu \mathrm{g} / \mathrm{mL}$}

Supplemental Figure 2.5. WI-38 exposed to BEAS-2B-conditioned media show limited to no presence of ND-MWCNT or MWCNT-7 in TEM images. Representative images shown suggest limited presence of ND-MWCNT or MWCNT-7, hence downstream effects are likely to be a result of cell signaling molecules in the conditioned media. 
CCL-2

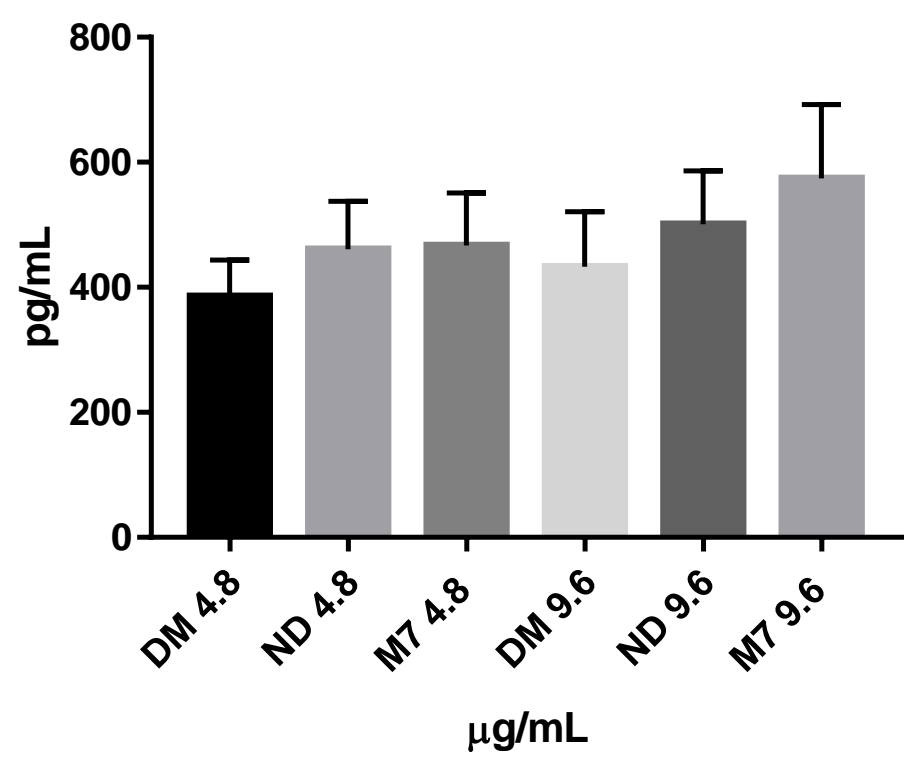

Supplemental Figure 2.6. Direct exposure to ND-MWCNT or MWCNT-7 did not induce CCL2 production in BEAS-2B. BEAS-2B media samples were collected 24 hours after exposure to 4.8 or $9.6 \mu \mathrm{g} / \mathrm{mL} \mathrm{DM}$, ND-MWCNT, or MWCNT-7, spun down to remove debris and extraneous CNT, and assayed by ELISA in three independent experiments. Results suggest that CCL2 production is not altered in BEAS-2B regardless of exposure. 


\section{PDGF-A}

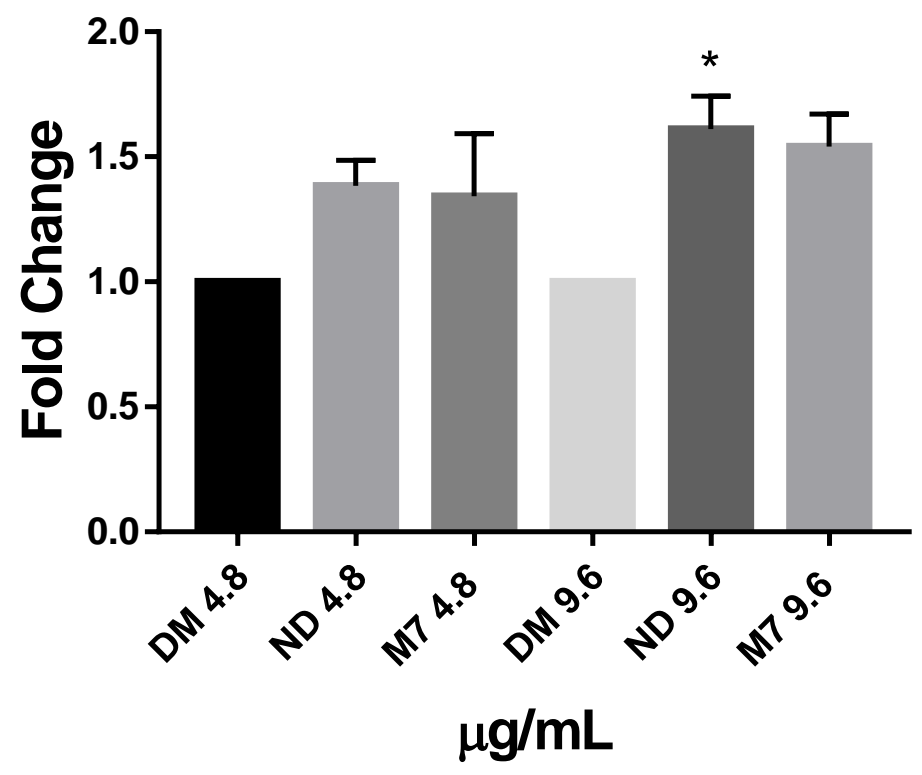

Supplemental Figure 2.7. BEAS-2B exposed to ND-MWCNT or MWCNT-7 increase gene expression of

PDGF-A. BEAS-2B were exposed to 4.8 or $9.6 \mu \mathrm{g} / \mathrm{mL}$ DM, ND-MWCNT, or MWCNT-7 over 24 hours.

Total RNA was collected, cDNA created, and qRT-PCR performed to analyze gene expression changes

after CNT exposure in three independent experiments. Results suggest that ND-MWCNT may

significantly increase expression of PDGF-A at $9.6 \mu \mathrm{g} / \mathrm{mL}(*, \mathrm{p}<0.05)$. 


\section{PDGF-AA}

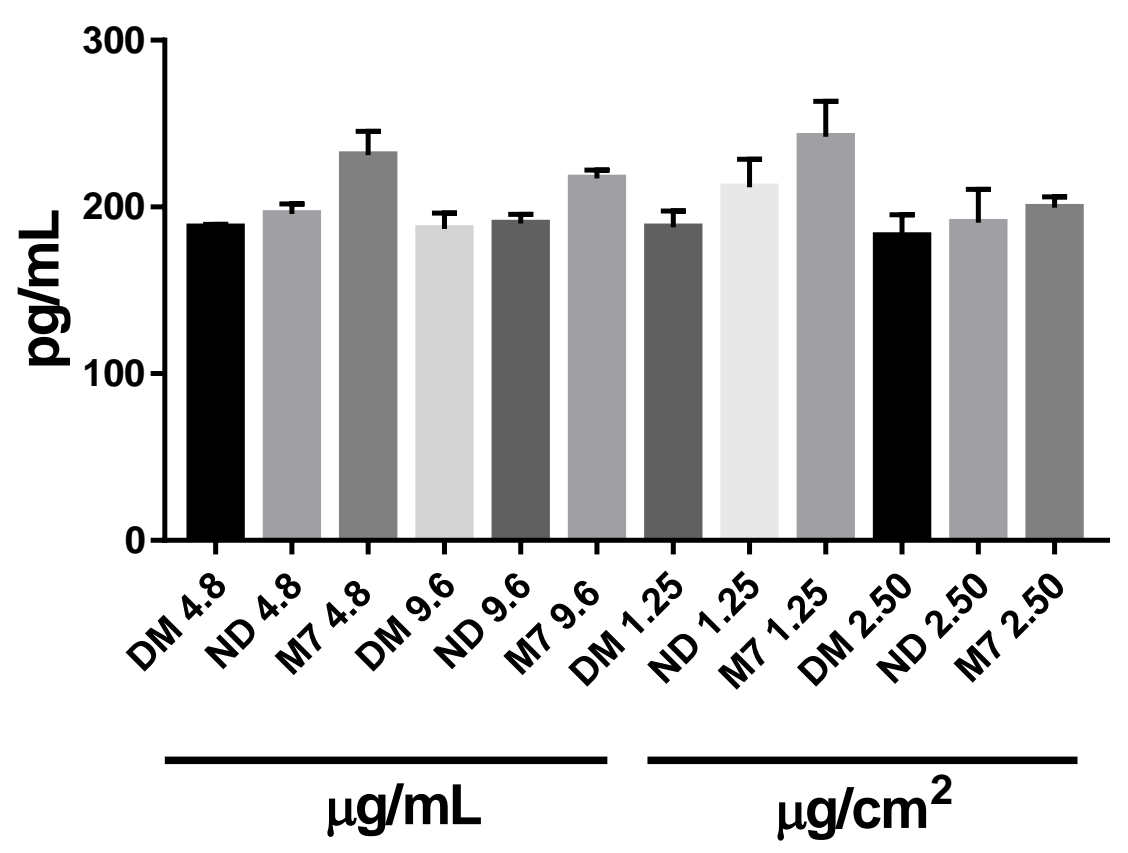

Supplemental Figure 2.8. BEAS-2B protein expression of PDGF-AA is not significant across

concentration-based and surface area-based exposures to ND-MWCNT or MWCNT-7. Media samples

were collected 24 hours after exposure to 4.8 or $9.6 \mu \mathrm{g} / \mathrm{mL}$, or 1.25 or $2.50 \mu \mathrm{g} / \mathrm{cm}^{2} \mathrm{DM}$, ND-MWCNT, or MWCNT-7, spun down to remove debris and extraneous CNT, and assayed by ELISA. Results suggest

that there are no significant changes between exposure conditions or treatments. ${ }^{*}, \mathrm{p}<0.05$ 
A

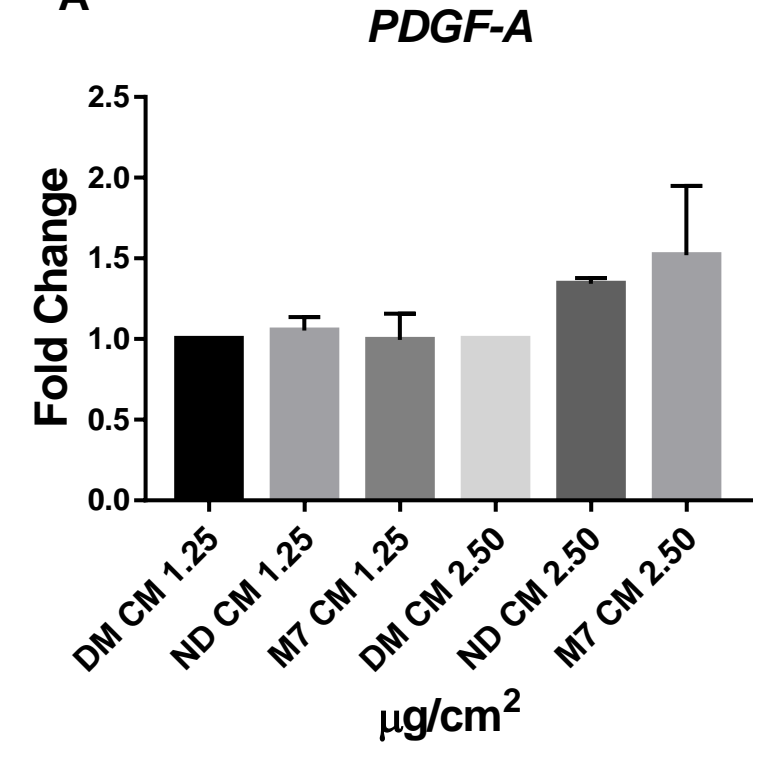

C

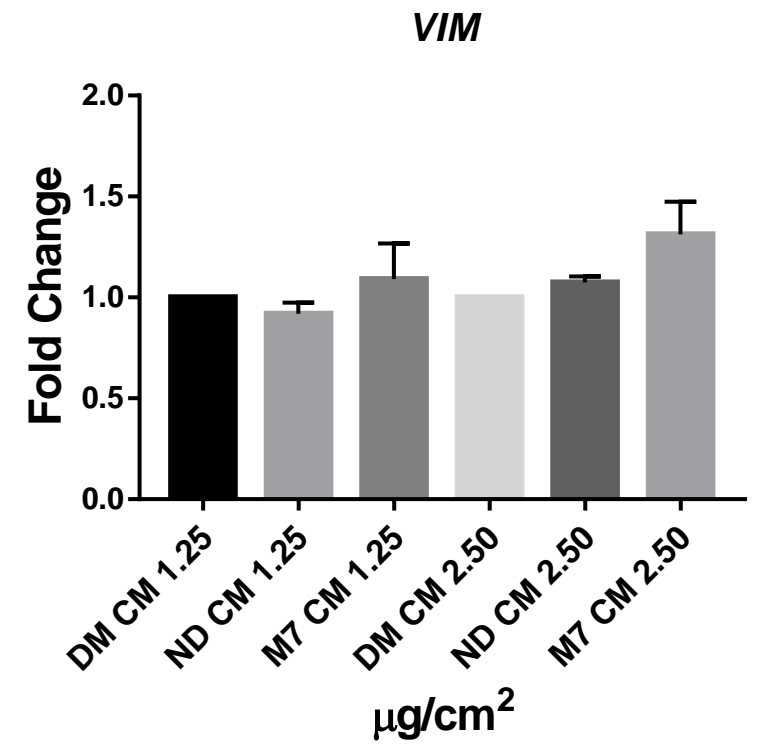

B

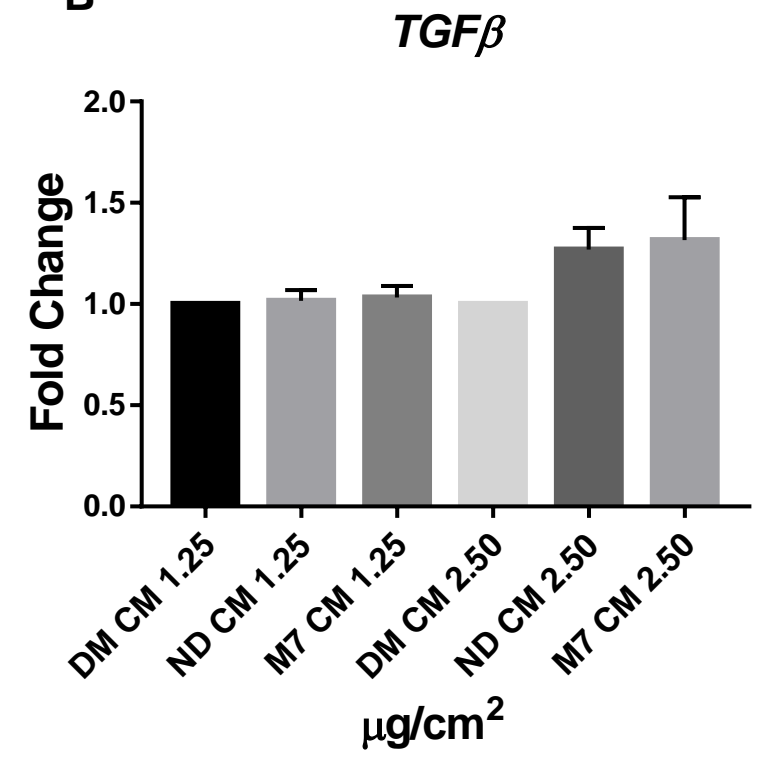

D

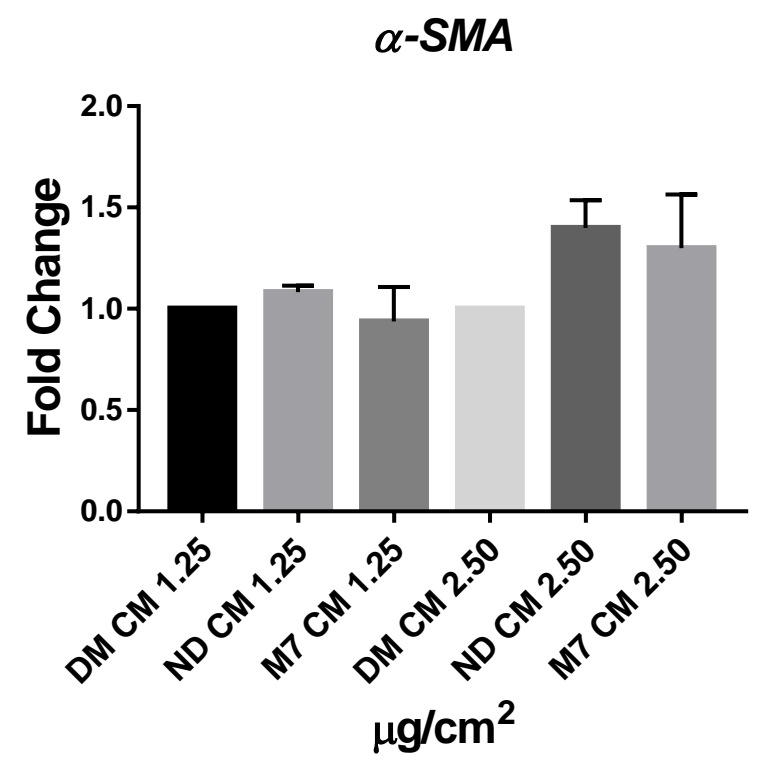

Supplemental Figure 2.9. Changing doses did not lead to significant cell responses in WI-38. An

attempt to normalize exposures utilizing surface area dosing instead of concentration based dosing did not change gene expression of $\alpha$-SMA, TGF $\beta$, Vimentin, or PDGF-A in WI-38, suggesting that conditioned media was ineffective for the study of WI-38 activity. 


\section{Supplemental Results:}

\subsection{Direct exposure of BEAS-2B with ND-MWCNT and MWCNT-7 are cytotoxic at high exposures.}

A CellTiter $96^{\circledR}$ Aqueous One (MTS) assay was carried out in three independent experiments to determine the effects of ND-MWCNT and MWCNT-7 on BEAS-2B and establish exposure doses for further study. BEAS-2B were exposed to $1.2,2.4,4.8,9.6,19.2$, or $38.4 \mu \mathrm{g} / \mathrm{mL}$ DM, ND-MWCNT or MWCNT-7 for $24 \mathrm{~h}$. No significant differences were observed between particles within the same exposure dose. BEAS-2B exposed to $19.2 \mu \mathrm{g} / \mathrm{mL}$ ND-MWCNT exhibited significant cytotoxicity compared to control ( $p<0.0018$ ), as well as $38.4 \mu \mathrm{g} / \mathrm{mL}$ ND-MWCNT and MWCNT-7 compared to control ( $p<$ $0.0001, p<0.0014)$ (Supplemental Figure 2.1.). These doses were subsequently removed from further study as we were not interested in studying significantly cytotoxic exposures in BEAS-2B. Since we were planning to use a conditioned media approach, we decided to focus on the 4.8 and $9.6 \mu \mathrm{g} / \mathrm{mL}$ doses as well, with the assumption that they would elicit a larger cytokine response in BEAS-2B that could elicit cellular responses in WI-38. 2 ..

\subsection{TEM assessment suggests that BEAS-2B interact with ND-MWCNT and MWCNT-7.}

BEAS-2B were exposed to 4.8 or $9.6 \mu \mathrm{g} / \mathrm{cm}^{2}$ DM, ND-MWCNT or MWCNT-7 for $24 \mathrm{~h}$ and prepared for assessment by TEM. ND-MWCNT and MWCNT-7 were primarily found in bundles or clumps that appeared to be endocytosed and found within vesicles (Supplemental Figure 2.2. and 2.3.).

\subsection{Direct exposure to ND-MWCNT or MWCNT-7 did not alter CCL2 protein production in BEAS-2B.}

Similar to the co-culture system, we wished to determine if BEAS-2B would produce CCL2 in monoculture conditioned media, especially upon using increased exposure doses to ND-MWCNT and MWCNT-7 compared to the co-culture experiments. Results suggested BEAS-2B did not significantly alter their CCL2 production regardless or exposure or dose as assessed using conditioned media in three independent ELISAs (Supplemental Figure 2.4.). 


\subsection{Direct exposure to ND-MWCNT or MWCNT-7 increase PDGF-A gene expression, but not protein}

production in BEAS-2B.

Since CCL2 did not appear to be induced by ND-MWCNT or MWCNT-7 in BEAS-2B, we chose to assess a different protein commonly associated with fibrogenic signaling, platelet-derived growth factor-AA (PDGF-AA). PDGF-A gene expression was increased over 1.5 fold in BEAS-2B after exposure to NDMWCNT or MWCNT-7, but only ND-MWCNT increase was found to be statistically significant by OneWay ANOVA analysis from three independent experiments (Supplemental Figure 2.7. A). Based upon this, a preliminary PDGF-AA ELISA was later performed to determine if protein production followed suit. However, results suggested that regardless of concentration or surface area-based dose, PDGF-AA protein expression did not significantly change (Supplemental Figure 2.7. B).

\subsection{BEAS-2B conditioned media applied to WI-38 show limited to no presence of ND-MWCNT or}

\section{MWCNT-7.}

Since co-culture of SAEC or BEAS-2B and WI-38 were found to be limited by cell type, cell viability, and media compatibility issues, we proposed the use of a 1:1 conditioned media approach in which we would expose BEAS-2B $24 \mathrm{~h}$ to 4.8 or $9.6 \mu \mathrm{g} / \mathrm{mL}$ DM, ND-MWCNT, or MWCNT-7, centrifuge the media at maximum speed in a chilled centrifuge, and apply conditioned media (CM) in a 1:1 ratio with fresh EMEM to WI-38 for $48 \mathrm{~h}$ prior to further assessment (Supplemental Figure 2.4.). TEM images of WI-38 exposed to CM over $48 \mathrm{~h}$ suggests limited or no presence of ND-MWCNT or MWCNT-7 within the cells, suggesting that any observed downstream effects were likely to be from soluble cell signaling molecules produced by the BEAS-2B (Supplemental Figure 2.5.).

\subsection{Surface area-based dosing did not lead to significant responses in WI-38}

While trouble-shooting the conditioned media approach, it became apparent that if one considered changing the amount of media applied to BEAS-2B to "concentrate" the cytokines within the conditioned media for instance, one would also concomitantly change the total $\mu \mathrm{g}$ of CNT applied to the 
cells as well. While this may appear obvious, exposing cells to concentration-based dosing was and still is a publishable methodology in nanotoxicology. However, this method lends itself to inherent issues if a researcher changes the surface of the plate he or she executes experiments in or changes the total volume of media applied to cells during an exposure. With this in mind, as described in the methods of this chapter, surface area based dosing was utilized. This change increased exposures from $4.8 \mu \mathrm{g} / \mathrm{mL}$ $\left(0.69 \mu \mathrm{g} / \mathrm{cm}^{2}\right)$ and $9.6 \mu \mathrm{g} / \mathrm{mL}\left(1.37 \mu \mathrm{g} / \mathrm{cm}^{2}\right)$ in $60 \mathrm{~mm}$ dishes with $3 \mathrm{~mL}$ media/dish to $1.25 \mu \mathrm{g} / \mathrm{cm}^{2}$ and $2.50 \mu \mathrm{g} / \mathrm{cm}^{2}$ which reflected exposures of BEAS-2B assayed by a pro-inflammatory cytokine kit. In order to assess if changing the dosing scheme would elicit a subsequent response in WI-38 exposed to BEAS2B conditioned media from 1.25 and $2.50 \mu \mathrm{g} / \mathrm{cm}^{2}$ CNT exposures, qRT-PCR for $\alpha-S M A$, vimentin, PDGFAA, and TGF $\beta 1$ was carried out on RNA from two independent experiments. Results showed no significant changes in gene expression for any of the genes regardless of exposure. With this in mind, the next logical step was to search for a method that was functional, reproducible, and gave careful consideration to dosimetry regardless of cell dish surface area.

\section{Supplemental Methods:}

\subsection{Conditioned Media Experimental Design}

BEAS-2B were exposed to 4.8 or $9.6 \mu \mathrm{g} / \mathrm{mL}$ DM, ND-MWCNT, or MWCNT-7 for $24 \mathrm{~h}$. Media was collected and centrifuged at maximum speed 8 minutes, decanted into another microfuge tube, and centrifuged at maximum speed an additional 8 minutes. Media was then applied to WI-38 in 1:1 ratio with fresh EMEM and assessed $48 \mathrm{~h}$ later for various endpoints. Later, the same process was followed, but BEAS-2B were treated with 1.25 or $2.50 \mu \mathrm{g} / \mathrm{cm}^{2}$.

\subsection{Transmission Electron Microscopy}

BEAS-2B interaction with and engulfment of ND-MWCNT and MWCNT-7 were analyzed by TEM. BEAS2B were grown to confluence and exposed to 4.8 or $9.6 \mu \mathrm{g} / \mathrm{mL} \mathrm{DM}$, ND-MWCNT, or MWCNT-7 for $24 \mathrm{~h}$. Additionally, WI-38 exposed to BEAS-2B conditioned media were assessed to check if CNT remained in 
conditioned media applied to WI-38 after centrifugation. Cells were trypsinized with Trypsin-EDTA (Gibco, Waltham, MA) per manufacturer guidelines and harvested by centrifugation at $400 \times \mathrm{g}$ for $5 \mathrm{~min}$. Cells were fixed in Karnovksy's fixative (2.5\% glutaraldehyde and 3\% paraformaldehyde in $0.1 \mathrm{M}$ sodium cacodylate, $\mathrm{pH} 7.4$ ), and $4 \%$ agarose was added to the pellet and lightly agitated such that agarose surrounded the pellet. Excess solid agarose was trimmed away placed in fresh Karnovsky's fixative over a day. Pellets were post-fixed in $1 \%$ osmium tetraoxide, and stained with $1 \%$ tannic acid and $0.5 \%$ uranyl acetate. The cells were dehydrated by sequential washings in $50,70,90$, and $100 \%$ ethanol and embedded in LX-112 (Ladd; Williston, VT). Ultrathin sections $70 \mathrm{~nm}$ thick were cut using a Leica UC7 ultramicrotome, placed on copper mesh grids, stained with $4 \%$ uranyl acetate ad lead citrate, and examined with a JEOL JEM-1400 transmission electron microscope (Peabody, MA). Images were taken using a mid-mount CCD digital camera system (Advanced Microscopy Techniques Corporation XR81M-B camera, Woburn, MA).

\subsection{Dose response cytotoxicity assay using CellTiter 96 Aqueous One Solution}

A dose-response assessment of cellular proliferation was carried out by seeding BEAS-2B in 96-well plates for $24 \mathrm{~h}$, followed by exposure to DM, ND-MWCNT, or MWCNT-7 for $24 \mathrm{~h}$ at 1.2, 2.4, 4.8, 9.6, 19.2 or $38.4 \mu \mathrm{g} / \mathrm{mL}$. $20 \mu \mathrm{L}$ of CellTiter $96^{\circledR}$ Aqueous One Solution (Promega; Madison, WI) was added to each well during the last 2 hours of exposure. Absorbance was read at $490 \mathrm{~nm}$ using a BioTek Synergy H1 plate reader (Winooski, VT). Three independent experiments were performed; treatment groups were averaged by technical triplicate and compared by One-Way ANOVA analysis and Tukey's Multiple Comparison test.

\subsection{Enzyme Linked Immunosorbent Assay}

Media was collected from BEAS-2B directly exposed to 4.8 or $9.6 \mu \mathrm{g} / \mathrm{mL}$, and later, 1.25 or $2.50 \mu \mathrm{g} / \mathrm{cm}^{2}$ centrifuged at max speed, and assayed in triplicate for CCL2. Conditioned media from each biological 
replicate were collected and assayed in triplicate for CCL2 and PDGF-AA expression levels using DuoSet ELISA Development Systems (R\&D Systems, Minneapolis, MN) according to manufacturer's protocol.

\subsection{Dosimetry of ND-MWCNT and MWCNT-7 in in vitro systems}

The conditioned media system and other in vitro methodologies employed in Chapter 3 also benefitted from a better understanding of surface area based dosimetry. While trouble-shooting the conditioned media project presented within this supplementary information and Chapter 3 , it became clear that surface area dosing would be essential to improving and normalizing results regardless of the surface area of the dish used experiment to experiment. Calculations below show how we arrived at using 1.25 and $2.50 \mathrm{\mu g} / \mathrm{cm}^{2}$ doses in BEAS-2B and how different concentration-based and surface area based doses are depending upon the surface area of a dish, which could have significant implications in how we interpret and design nanotoxicology studies.

Assuming one treats a 48 well plate with a surface area of $0.95 \mathrm{~cm}^{2}$ with $250 \mu \mathrm{L}$ of media with a concentration of $4.8 \mu \mathrm{g} / \mathrm{mL}$ (which is what was used for the MSD pro-inflammatory plate experiment in Chapter 3), see below:

Eq. $1 \frac{4.8 \mu \mathrm{g}}{\mathrm{mL}} \times 0.250 \mathrm{~mL}=1.2 \mu \mathrm{g} \quad \frac{1.2 \mu \mathrm{g}}{0.95 \mathrm{~cm}^{2}} \approx 1.26 \frac{\mu \mathrm{g}}{\mathrm{cm}^{2}}$

I decided to $1.25 \mu \mathrm{g} / \mathrm{cm}^{2}$ based upon ease of understanding in light of results for $9.6 \mu \mathrm{g} / \mathrm{mL}$

Eq. $2 \frac{9.6 \mu \mathrm{g}}{\mathrm{mL}} \times 0.250 \mathrm{~mL}=2.4 \mu \mathrm{g} \quad \frac{2.4 \mu \mathrm{g}}{0.95 \mathrm{~cm}^{2}}=2.50 \frac{\mu \mathrm{g}}{\mathrm{cm}^{2}}$

The doses used for the remainder of these studies and in Chapter 3 were 1.25 and $2.50 \mu \mathrm{g} / \mathrm{cm}^{2}$, especially under the assumption that these doses were required to elicit a pro-inflammatory or profibrotic signaling response in BEAS-2B that could also activate fibroblasts.

\subsection{Enzyme Linked Immunosorbent Assay}

Media was collected from BEAS-2B exposed to 4.8 or $9.6 \mu \mathrm{g} / \mathrm{mL}$ or 1.25 or $2.50 \mu \mathrm{g} / \mathrm{cm}^{2}$, centrifuged at max speed, and assayed in technical triplicate. Conditioned media from each biological replicate were 
collected and assayed in triplicate for $\mathrm{C}-\mathrm{C}$ motif chemokine 2 (CCL2) and platelet-derived growth factorAA protein expression levels using DuoSet ELISA Development Systems (R\&D Systems, Minneapolis, MN) according to manufacturer's protocol.

\subsection{Quantitative Real-Time PCR}

RNA was isolated from WI-38 treated with BEAS-2B conditioned media exposed to 1.25 or $2.50 \mu \mathrm{g} / \mathrm{cm}^{2}$ using a Qiagen RNeasy Mini Kit according to the manufacturer's protocol (Qiagen, Hilden, Germany), and concentration was determined using a NanoDrop 1000 Spectrophotometer (NanoDrop Technologies, Wilmington, DE). $1 \mu \mathrm{g}$ of RNA per sample was converted into complementary DNA (cDNA) using a High Capacity cDNA Reverse Transcription Kit from Applied Biosystems (Life Technologies, Carlsbad, CA). The manufacturer's suggested thermal cycling conditions were used as follows: $25^{\circ} \mathrm{C}$ for 10 minutes, 2 cycles of $37^{\circ} \mathrm{C}$ for 60 minutes, and $85^{\circ} \mathrm{C}$ for 5 seconds, and held at $4^{\circ} \mathrm{C}$. Quantitative realtime PCR reactions were performed using an Applied Biosystems 7500 Real-Time PCR instrument. As per manufacturer guidelines, cDNA was diluted 1:10 in nuclease free water and mixed with TaqMan Gene Expression Assays diluted 1:10 in TaqMan Universal PCR Master Mix, all from Applied Biosystems. Gene expression assay numbers utilized are provided as follows: 18S (Hs99999901_s1), ACT2A (Hs00426835_g1), VIM (Hs00958111_m1), PDGFA (Hs00964426_m1), and TGFß1 (Hs00998133_m1). qRT-PCR analysis for each sample was carried out in duplicate and normalized to the $18 \mathrm{~S}$ housekeeping gene. Thermal cycling conditions were as follows: $50^{\circ} \mathrm{C}$ for $2 \mathrm{~min}, 95^{\circ} \mathrm{C}$ for $10 \mathrm{~min}$, followed by 40 cycles of $95^{\circ} \mathrm{C}$ for $15 \mathrm{~s}$ and $60^{\circ} \mathrm{C}$ for $10 \mathrm{~min}$. Fold change was determined using the $2^{-\Delta \Delta \mathrm{Ct}}$ method.

\subsection{Statistical Analysis}

Statistical analyses for the data were performed using GraphPad Prism 7.00. One-Way ANOVA tests comparing across all means for sample groups were utilized, and Tukey's Multiple Comparison's Test were used to determine significance between treatment groups, unless otherwise noted. 


\section{References}

1. National Nanotechnology Initiative. 2013; Available from: http://www.nano.gov/.

2. Nel, A., et al., Toxic Potential of Materials at the Nanolevel. Science, 2006. 311(5761): p. 622627.

3. Castranova, V., Overview of current toxicological knowledge of engineered nanoparticles. Journal of Occupational and Environmental Medicine, 2011. 53: p. S14-S17.

4. Oberdörster, G., E. Oberdörster, and J. Oberdörster, Nanotoxicology: An Emerging Discipline Evolving from Studies of Ultrafine Particles. Environmental Health Perspectives, 2005. 113(7): p. 823-839.

5. Nel, A.E., et al., A Multi-Stakeholder Perspective on the Use of Alternative Test Strategies for Nanomaterial Safety Assessment. ACS nano, 2013. 7(8): p. 6422-6433.

6. Collins, F.S., G.M. Gray, and J.R. Bucher, Transforming Environmental Health Protection. Science (New York, N.Y.), 2008. 319(5865): p. 906-907.

7. Teeguarden, J.G., et al., Particokinetics In Vitro: Dosimetry Considerations for In Vitro Nanoparticle Toxicity Assessments. Toxicological Sciences, 2007. 95(2): p. 300-312.

8. National Research Council, C.o.T.T., et al., Toxicity testing in the 21st century: $a$ vision and a strategy. 2007, Washington, DC: National Academies Press.

9. Russell, W.M.S., R.L. Burch, and C.W. Hume, The principles of humane experimental technique. 1959.

10. Snyder-Talkington, B.N., et al., New Perspectives for In Vitro Risk Assessment of Multi-Walled Carbon Nanotubes: Application of Coculture and Bioinformatics. Journal of toxicology and environmental health. Part B, Critical reviews, 2012. 15(7): p. 468-492.

11. Nel, A.E., Implementation of alternative test strategies for the safety assessment of engineered nanomaterials. J Intern Med, 2013. 274(6): p. 561-77. 
12. Sayes, C.M., K.L. Reed, and D.B. Warheit, Assessing toxicity of fine and nanoparticles: comparing in vitro measurements to in vivo pulmonary toxicity profiles. Toxicological sciences, 2007. 97(1): p. $163-180$.

13. Seagrave, J., J.L. Mauderly, and S.K. Seilkop, In vitro relative toxicity screening of combined particulate and semivolatile organic fractions of gasoline and diesel engine emissions. Journal of Toxicology and Environmental Health, Part A, 2003. 66(12): p. 1113-1132.

14. Boublil, L., L. Martinon, and A. Baeza-Squiban, The Secretome of Human Bronchial Epithelial Cells Exposed to Fine Atmospheric Particles Induces Fibroblast Proliferation. Challenges (20781547), 2013. 4(2): p. 188-200.

15. Hussain, S., et al., Inflammasome activation in airway epithelial cells after multi-walled carbon nanotube exposure mediates a profibrotic response in lung fibroblasts. Particle and Fibre Toxicology, 2014. 11: p. 28-28.

16. Park, A.-M., et al., Conditioned media from lung cancer cell line A549 and PC9 inactivate pulmonary fibroblasts by regulating protein phosphorylation. Archives of Biochemistry and Biophysics, 2012. 518(2): p. 133-141.

17. Snyder-Talkington, B.N., et al., Multi-walled carbon nanotube-induced gene expression in vitro: Concordance with in vivo studies. TOXICOLOGY, 2015. 328: p. 66-74.

18. Fromigué, O., et al., Gene expression profiling of normal human pulmonary fibroblasts following coculture with non-small-cell lung cancer cells reveals alterations related to matrix degradation, angiogenesis, cell growth and survival. Oncogene, 2003. 22(52): p. 8487-8497.

19. Snyder-Talkington, B.N., et al., Multi-walled carbon nanotubes induce human microvascular endothelial cellular effects in an alveolar-capillary co-culture with small airway epithelial cells. Particle and Fibre Toxicology, 2013. 10: p. 35-35. 
20. Rohr, S.M., A. Heires, and A. Floreani, BASAL LEVELS OF NERVE GROWTH FACTOR ARE HIGHER IN CO-CULTURED HUMAN A-549 TYPE II ALVEOLAR EPITHELIAL CELLS WITH HUMAN FIBROBLASTS THAN IN EITHER CELL LINE CULTURED ALONE. Chest, 2005. 128(4): p. 168.

21. Nakamura, Y., et al., Bronchial epithelial cells regulate fibroblast proliferation. AJP - Lung Cellular and Molecular Physiology, 1995. 269(3): p. 377.

22. Skibinski, G., J.S. Elborn, and M. Ennis, Bronchial epithelial cell growth regulation in fibroblast cocultures: the role of hepatocyte growth factor. American Journal of Physiology - Lung Cellular and Molecular Physiology, 2007. 293(1): p. 69-76.

23. Prasad, S., C.M. Hogaboam, and G. Jarai, Deficient repair response of IPF fibroblasts in a coculture model of epithelial injury and repair. Fibrogenesis \& tissue repair, 2014. 7(1): p. 7-7.

24. Li, R.B., et al., Surface Charge and Cellular Processing of Covalently Functionalized Multiwall Carbon Nanotubes Determine Pulmonary Toxicity. ACS NANO, 2013. 7(3): p. 2352-2368.

25. Bauer, R.N., et al., Interaction with epithelial cells modifies airway macrophage response to ozone. Am J Respir Cell Mol Biol, 2015. 52(3): p. 285-94.

26. Lehmann, A.D., et al., An in vitro triple cell co-culture model with primary cells mimicking the human alveolar epithelial barrier. European Journal of Pharmaceutics and Biopharmaceutics, 2011. 77(3): p. 398-406.

27. Rothen-Rutishauser, B.M., S.G. Kiama, and P. Gehr, A Three-Dimensional Cellular Model of the Human Respiratory Tract to Study the Interaction with Particles. American Journal of Respiratory Cell and Molecular Biology, 2005. 32(4): p. 281-289.

28. Sisler, J.D., et al., Small airway epithelial cells exposure to printer-emitted engineered nanoparticles induces cellular effects on human microvascular endothelial cells in an alveolarcapillary co-culture model. Nanotoxicology, 2015. 9(6): p. 769-779. 
29. Lai, X., et al., Protein expression profiles of intestinal epithelial co-cultures: effect of functionalised carbon nanotube exposure. International journal of biomedical nanoscience and nanotechnology, 2013. 3(1-2): p. 10.1504/IJBNN.2013.054508.

30. Müller, L., et al., Oxidative stress and inflammation response after nanoparticle exposure: differences between human lung cell monocultures and an advanced three-dimensional model of the human epithelial airways. Journal of The Royal Society Interface, 2009.

31. Greer, R.M., et al., Epithelial-mesenchymal co-culture model for studying alveolar morphogenesis. Organogenesis, 2014. 10(4): p. 340-349.

32. Pageau, S.C., et al., The effect of stromal components on the modulation of the phenotype of human bronchial epithelial cells in 3D culture. Biomaterials, 2011. 32(29): p. 7169-7180.

33. Sauer, U.G., et al., In vivo-in vitro comparison of acute respiratory tract toxicity using human 3D airway epithelial models and human A549 and murine $3 T 3$ monolayer cell systems. Toxicology in vitro : an international journal published in association with BIBRA, 2013. 27(1): p. 174-190.

34. Huh, D., G.A. Hamilton, and D.E. Ingber, From Three-Dimensional Cell Culture to Organs-onChips. Trends in cell biology, 2011. 21(12): p. 745-754.

35. Huh, D., et al., <strong $>$ Reconstituting Organ-Level Lung Functions on a Chip $</$ strong $>$. Science, 2010. 328(5986): p. 1662-1668.

36. Hinderliter, P.M., et al., ISDD: A computational model of particle sedimentation, diffusion and target cell dosimetry for in vitro toxicity studies. Particle and Fibre Toxicology, 2010. 7(1): p. 120.

37. Cohen, J.M., J.G. Teeguarden, and P. Demokritou, An integrated approach for the in vitro dosimetry of engineered nanomaterials. PARTICLE AND FIBRE TOXICOLOGY, 2014. 11(1): p. 2020. 
38. DeLoid, G., et al., Estimating the effective density of engineered nanomaterials for in vitro dosimetry. NATURE COMMUNICATIONS, 2014. 5: p. 3514.

39. Alarifi, S. and D. Ali, Mechanisms of Multi-walled Carbon Nanotubes-Induced Oxidative Stress and Genotoxicity in Mouse Fibroblast Cells. Int J Toxicol, 2015. 34(3): p. 258-65.

40. Porter, D.W., et al., Mouse pulmonary dose-and time course-responses induced by exposure to multi-walled carbon nanotubes. Toxicology, 2010. 269(2): p. 136-147.

41. He, X., et al., Multiwalled Carbon Nanotubes Induce a Fibrogenic Response by Stimulating Reactive Oxygen Species Production, Activating NF-kB Signaling, and Promoting Fibroblast-toMyofibroblast Transformation. Chemical Research in Toxicology, 2011. 24(12): p. 2237-2248.

42. Wang, X., et al., The Dispersal State of Multi-walled Carbon Nanotubes Elicits Pro-Fibrogenic Cellular Responses that Correlate with Fibrogenesis Biomarkers and Fibrosis in the Murine Lung. ACS nano, 2011. 5(12): p. 9772-9787.

43. Vietti, G., D. Lison, and S. van den Brule, Mechanisms of lung fibrosis induced by carbon nanotubes: towards an Adverse Outcome Pathway (AOP). Particle and Fibre Toxicology, 2016. 13(1): p. 1-23.

44. Gangwal, S., et al., Informing Selection of Nanomaterial Concentrations for ToxCast in Vitro Testing Based on Occupational Exposure Potential. Environmental Health Perspectives, 2011. 119(11): p. 1539-1546.

45. Oberdörster, G., Nanotoxicology: in Vitro-in Vivo Dosimetry. Environmental Health Perspectives, 2012. 120(1): p. A13-A13.

46. Han, J.H., et al., Monitoring multiwalled carbon nanotube exposure in carbon nanotube research facility. Inhal Toxicol, 2008. 20(8): p. 741-9. 
47. Dahm, M.M., et al., Occupational exposure assessment in carbon nanotube and nanofiber primary and secondary manufacturers: mobile direct-reading sampling. Ann Occup Hyg, 2013. 57(3): p. 328-44.

48. Chen, B.T., et al., Multi-walled carbon nanotubes: sampling criteria and aerosol characterization. Inhalation toxicology, 2012. 24(12): p. 798-820.

49. Oberdörster, G., et al., Principles for characterizing the potential human health effects from exposure to nanomaterials: elements of a screening strategy. Particle and Fibre Toxicology, 2005. 2(1): p. 1-35.

50. Singh, S., et al., Endocytosis, oxidative stress and IL-8 expression in human lung epithelial cells upon treatment with fine and ultrafine TiO2: role of the specific surface area and of surface methylation of the particles. Toxicol Appl Pharmacol, 2007. 222(2): p. 141-51.

51. Sager, T.M. and V. Castranova, Surface area of particle administered versus mass in determining the pulmonary toxicity of ultrafine and fine carbon black: comparison to ultrafine titanium dioxide. Particle and Fibre Toxicology, 2009. 6: p. 15-15.

52. Monteiller, C., et al., The pro-inflammatory effects of low-toxicity low-solubility particles, nanoparticles and fine particles, on epithelial cells in vitro: the role of surface area. Occup Environ Med, 2007. 64(9): p. 609-15.

53. Stoeger, T., et al., Instillation of Six Different Ultrafine Carbon Particles Indicates a Surface Area Threshold Dose for Acute Lung Inflammation in Mice. Environmental Health Perspectives, 2006. 114(3): p. 328-333.

54. Birch, M.E., et al., Properties that Influence the Specific Surface Areas of Carbon Nanotubes and Nanofibers. The Annals of occupational hygiene, 2013. 57(9): p. 1148-1166.

55. Naseh, M.V., et al., Functionalization of carbon nanotubes using nitric acid oxidation and DBD plasma. World Academy of Science, Engineering and Technology, 2009. 49: p. 177-179. 
56. Chakraborty, S., et al., Surface Area Measurement of Functionalized Single-Walled Carbon Nanotubes. The Journal of Physical Chemistry B, 2006. 110(49): p. 24812-24815.

57. Erdely, A., et al., Carbon nanotube dosimetry: from workplace exposure assessment to inhalation toxicology. Particle and Fibre Toxicology, 2013. 10: p. 53-53.

58. lijima, S., Helical microtubules of graphitic carbon. nature, 1991. 354(6348): p. 56-58.

59. Oberlin, A., M. Endo, and T. Koyama, Filamentous growth of carbon through benzene decomposition. Journal of Crystal Growth, 1976. 32(3): p. 335-349.

60. Zhang, X., et al., Ultrastrong, stiff, and lightweight Carbon-Nanotube fibers. Advanced Materials, 2007. 19(23): p. 4198-4201.

61. Tsuruoka, S., F.R. Cassee, and V. Castranova, A new approach to design safe CNTs with an understanding of redox potential. PARTICLE AND FIBRE TOXICOLOGY, 2013. 10(1): p. 44-44.

62. Karousis, N., N. Tagmatarchis, and D. Tasis, Current progress on the chemical modification of carbon nanotubes. Chemical Reviews, 2010. 110(9): p. 5366-5397.

63. Vardharajula, S., et al., Functionalized carbon nanotubes: biomedical applications. International Journal of Nanomedicine, 2012. 7: p. 5361-5374.

64. Duclaux, L., Review of the doping of carbon nanotubes (multiwalled and single-walled). Carbon, 2002. 40(10): p. 1751-1764.

65. Bianco, A., K. Kostarelos, and M. Prato, Applications of carbon nanotubes in drug delivery. Current Opinion in Chemical Biology, 2005. 9(6): p. 674-679.

66. Chatterjee, N., et al., Potential toxicity of differential functionalized multiwalled carbon nanotubes (MWCNT) in human cell line (BEAS2B) and Caenorhabditis elegans. J Toxicol Environ Health A, 2014. 77(22-24): p. 1399-408. 
67. Ursini, C.L., et al., Comparative cyto-genotoxicity assessment of functionalized and pristine multiwalled carbon nanotubes on human lung epithelial cells. Toxicol In Vitro, 2012. 26(6): p. 831-40.

68. Li, R., et al., The Surface Charge and Cellular Processing of Covalently Functionalized Multiwall Carbon Nanotubes Determine Pulmonary Toxicity. ACS nano, 2013. 7(3): p. 2352-2368.

69. Dumortier, H., et al., Functionalized Carbon Nanotubes Are Non-Cytotoxic and Preserve the Functionality of Primary Immune Cells. Nano Letters, 2006. 6(7): p. 1522-1528.

70. Carrero-Sanchez, J., et al., Biocompatibility and toxicological studies of carbon nanotubes doped with nitrogen. Nano Letters, 2006. 6(8): p. 1609-1616.

71. Sager, T.M., et al., Effect of multi-walled carbon nanotube surface modification on bioactivity in the C57BL/6 mouse model. Nanotoxicology, 2014. 8(3): p. 317-327.

72. Mihalchik, A.L., et al., Effects of nitrogen-doped multi-walled carbon nanotubes compared to pristine multi-walled carbon nanotubes on human small airway epithelial cells. Toxicology, 2015. 333: p. 25-36.

73. Aqel, A., et al., Carbon nanotubes, science and technology part (I) structure, synthesis and characterisation. Arabian Journal of Chemistry, 2012. 5(1): p. 1-23.

74. Eatemadi, A., et al., Carbon nanotubes: properties, synthesis, purification, and medical applications. Nanoscale Research Letters, 2014. 9(1): p. 393-393.

75. Terrones, M., SCIENCE AND TECHNOLOGY OF THE TWENTY-FIRST CENTURY: Synthesis, Properties, and Applications of Carbon Nanotubes. Annual Review of Materials Research, 2003. 33(1): p. 419-501.

76. Dong, J., et al., Pathologic and molecular profiling of rapid-onset fibrosis and inflammation induced by multi-walled carbon nanotubes. ARCHIVES OF TOXICOLOGY, 2015. 89(4): p. 621-633. 
77. Mercer, R.R., et al., Pulmonary fibrotic response to aspiration of multi-walled carbon nanotubes. Particle and Fibre Toxicology, 2011. 8: p. 21-21.

78. Mercer, R.R., et al., Distribution and fibrotic response following inhalation exposure to multiwalled carbon nanotubes. Particle and Fibre Toxicology, 2013. 10: p. 33-33.

79. Mercer, R.R., et al., Extrapulmonary transport of MWCNT following inhalation exposure. Particle and Fibre Toxicology, 2013. 10: p. 38-38.

80. Porter, D.W., et al., Acute pulmonary dose-responses to inhaled multi-walled carbon nanotubes. Nanotoxicology, 2013. 7(7): p. 1179-1194.

81. Sargent, L.M., et al., Promotion of lung adenocarcinoma following inhalation exposure to multiwalled carbon nanotubes. Particle and Fibre Toxicology, 2014. 11: p. 3-3.

82. Siegrist, K.J., et al., Genotoxicity of multi-walled carbon nanotubes at occupationally relevant doses. Particle and Fibre Toxicology, 2014. 11: p. 6-6.

83. Kasai, T., et al., Thirteen-week study of toxicity of fiber-like multi-walled carbon nanotubes with whole-body inhalation exposure in rats. Nanotoxicology, 2015. 9(4): p. 413-422.

84. Takagi, A., et al., Induction of mesothelioma in p53+/-mouse by intraperitoneal application of multi-wall carbon nanotube. The Journal of toxicological sciences, 2008. 33(1): p. 105-116.

85. Fujitani, T., et al., Teratogenicity of multi-wall carbon nanotube (MWCNT) in ICR mice. J Toxicol Sci, 2012. 37(1): p. 81-9.

86. Stapleton, P.A., et al., Impairment of coronary arteriolar endothelium-dependent dilation after multi-walled carbon nanotube inhalation: a time-course study. International journal of molecular sciences, 2012. 13(11): p. 13781-13803.

87. Aragon, M., et al., MMP-9-Dependent Serum-Borne Bioactivity Caused by Multiwalled Carbon Nanotube Exposure Induces Vascular Dysfunction via the CD36 Scavenger Receptor. Toxicol Sci, 2016. 150(2): p. 488-98. 
88. Donaldson, K., et al., Asbestos, carbon nanotubes and the pleural mesothelium: a review of the hypothesis regarding the role of long fibre retention in the parietal pleura, inflammation and mesothelioma. Particle and Fibre Toxicology, 2010. 7: p. 5-5.

89. Poland, C.A., et al., Carbon nanotubes introduced into the abdominal cavity of mice show asbestos-like pathogenicity in a pilot study. Nat Nano, 2008. 3(7): p. 423-428.

90. Hwang, C.Y., Size and shape of airborne asbestos fibres in mines and mills. British Journal of Industrial Medicine, 1983. 40(3): p. 273-279.

91. Snyder-Talkington, B.N., et al., Systematic Analysis of Multiwalled Carbon Nanotube-Induced Cellular Signaling and Gene Expression in Human Small Airway Epithelial Cells. Toxicological Sciences, 2013. 133(1): p. 79-89.

92. Wang, L., et al., Neoplastic-like transformation effect of single-walled and multi-walled carbon nanotubes compared to asbestos on human lung small airway epithelial cells. Nanotoxicology, 2014. 8(5): p. 485-507.

93. Jia, G., et al., Cytotoxicity of carbon nanomaterials: single-wall nanotube, multi-wall nanotube, and fullerene. Environmental science \& technology, 2005. 39(5): p. 1378-1383.

94. Murphy, F.A., et al., Length-Dependent Retention of Carbon Nanotubes in the Pleural Space of Mice Initiates Sustained Inflammation and Progressive Fibrosis on the Parietal Pleura. The American Journal of Pathology, 2011. 178(6): p. 2587-2600.

95. Murphy, F.A., et al., The mechanism of pleural inflammation by long carbon nanotubes: interaction of long fibres with macrophages stimulates them to amplify pro-inflammatory responses in mesothelial cells. Particle and Fibre Toxicology, 2012. 9: p. 8-8.

96. Pauluhn, J., Subchronic 13-week inhalation exposure of rats to multiwalled carbon nanotubes: toxic effects are determined by density of agglomerate structures, not fibrillar structures. Toxicol Sci, 2010. 113(1): p. 226-42. 
97. Endo, M., et al., Applications of carbon nanotubes in the twenty-first century. Philosophical Transactions of the Royal Society of London. Series A: Mathematical, Physical and Engineering Sciences, 2004. 362(1823): p. 2223-2238.

98. Jain, S., et al., Toxicity of Multiwalled Carbon Nanotubes with End Defects Critically Depends on Their Functionalization Density. Chemical Research in Toxicology, 2011. 24(11): p. 2028-2039.

99. Fenoglio, I., et al., Structural defects play a major role in the acute lung toxicity of multiwall carbon nanotubes: physicochemical aspects. Chemical research in toxicology, 2008. 21(9): p. 1690-1697.

100. Tsuruoka, S., et al., Differentiation of chemical reaction activity of various carbon nanotubes using redox potential: Classification by physical and chemical structures. Carbon, 2015. 95: p. 302-308.

101. Muller, J., et al., Structural defects play a major role in the acute lung toxicity of multiwall carbon nanotubes: toxicological aspects. Chemical research in toxicology, 2008. 21(9): p. 1698-1705.

102. Hamilton, R.F., et al., Effect of MWCNT size, carboxylation, and purification on in vitro and in vivo toxicity, inflammation and lung pathology. Particle and Fibre Toxicology, 2013. 10: p. 57-57.

103. Hamilton, R.F., et al., Purification and sidewall functionalization of multiwalled carbon nanotubes and resulting bioactivity in two macrophage models. Inhalation toxicology, 2013. 25(4): p. 199-210.

104. Tabet, L., et al., Coating carbon nanotubes with a polystyrene-based polymer protects against pulmonary toxicity. Particle and Fibre Toxicology, 2011. 8: p. 3-3.

105. Mercer, R.R., et al., Distribution and persistence of pleural penetrations by multi-walled carbon nanotubes. Particle and Fibre Toxicology, 2010. 7: p. 28-28.

106. Occupational Exposure to Carbon Nanotubes and Nanofibers, N.I.f.O.S.a. Health, Editor. 2013. 
107. Pacurari, M., et al., Multi-Walled Carbon Nanotube-Induced Gene Expression in the Mouse Lung: Association with Lung Pathology. Toxicology and applied pharmacology, 2011. 255(1): p. 18-31.

108. Ding, L., et al., Molecular characterization of the cytotoxic mechanism of multiwall carbon nanotubes and nano-onions on human skin fibroblast. Nano Letters, 2005. 5(12): p. 2448-2464.

109. Pacurari, M., et al., Cell Permeability, Migration, and Reactive Oxygen Species Induced by MultiWalled Carbon Nanotubes in Human Microvascular Endothelial Cells. Journal of toxicology and environmental health. Part A, 2012. 75(2): p. 112-128.

110. Mishra, A., et al., Assessment of pulmonary fibrogenic potential of multiwalled carbon nanotubes in human lung cells. Journal of Nanomaterials, 2012. 2012: p. 4.

111. Snyder-Talkington, B.N., et al., System-based Identification of Toxicity Pathways Associated With Multi-Walled Carbon Nanotube-Induced Pathological Responses. Toxicology and applied pharmacology, 2013. 272(2): p. 476-489.

112. Sharifi, T., et al., Nitrogen doped multi walled carbon nanotubes produced by CVD-correlating XPS and Raman spectroscopy for the study of nitrogen inclusion. Carbon, 2012. 50(10): p. 35353541.

113. Ayala, P., et al., The doping of carbon nanotubes with nitrogen and their potential applications. Carbon, 2010. 48(3): p. 575-586.

114. Tsuruoka, S., et al., ROS evaluation for a series of CNTs and their derivatives using an ESR method with DMPO. Journal of physics. Conference series, 2013. 429(12029).

115. Zhao, M., et al., Differences in cytocompatibility and hemocompatibility between carbon nanotubes and nitrogen-doped carbon nanotubes. Carbon, 2011. 49(9): p. 3125-3133.

116. Elías, A.L., et al., Viability Studies of Pure Carbon-and Nitrogen-Doped Nanotubes with Entamoeba histolytica: From Amoebicidal to Biocompatible Structures. Small, 2007. 3(10): p. 1723-1729. 
117. Boncel, S., et al., Tunable chemistry and morphology of multi-wall carbon nanotubes as a route to non-toxic, theranostic systems. Biomaterials, 2011. 32(30): p. 7677-7686.

118. Porter, D., et al., A biocompatible medium for nanoparticle dispersion. Nanotoxicology, 2008. 2(3): p. 144-154.

119. Piao, C.Q., et al., Immortalization of human small airway epithelial cells by ectopic expression of telomerase. Carcinogenesis, 2005. 26(4): p. 725-731.

120. Apopa, P.L., et al., Iron oxide nanoparticles induce human microvascular endothelial cell permeability through reactive oxygen species production and microtubule remodeling. Particle and fibre toxicology, 2009. 6(1): p. 1.

121. Huerta-García, E., et al., Titanium dioxide nanoparticles induce strong oxidative stress and mitochondrial damage in glial cells. Free Radical Biology and Medicine, 2014. 73: p. 84-94.

122. Pichardo, S., et al., Oxidative stress responses to carboxylic acid functionalized single wall carbon nanotubes on the human intestinal cell line Caco-2. Toxicology in Vitro, 2012. 26(5): p. 672-677.

123. Shi, J., et al., Endothelial cell injury and dysfunction induced by silver nanoparticles through oxidative stress via IKK/NF-KB pathways. Biomaterials, 2014. 35(24): p. 6657-6666.

124. Zhang, Y. and B. Yan, Cell cycle regulation by carboxylated multiwalled carbon nanotubes through p53-independent induction of p21 under the control of the BMP signaling pathway. Chemical research in toxicology, 2012. 25(6): p. 1212-1221.

125. Hunter, T., Protein kinases and phosphatases: the yin and yang of protein phosphorylation and signaling. Cell, $1995.80(2)$ : p. 225-236.

126. Marshall, C., Specificity of receptor tyrosine kinase signaling: transient versus sustained extracellular signal-regulated kinase activation. Cell, 1995. 80(2): p. 179-185.

127. Kuzmany, H., et al., Functionalization of carbon nanotubes. Synthetic Metals, 2004. 141(1): p. 113-122. 
128. Meier, M.S., et al., Tearing open nitrogen-doped multiwalled carbon nanotubes. J. Mater. Chem., 2008. 18(35): p. 4143-4145.

129. Maldonado, S., S. Morin, and K.J. Stevenson, Structure, composition, and chemical reactivity of carbon nanotubes by selective nitrogen doping. Carbon, 2006. 44(8): p. 1429-1437.

130. Kundu, S., et al., Electrocatalytic activity and stability of nitrogen-containing carbon nanotubes in the oxygen reduction reaction. The Journal of Physical Chemistry C, 2009. 113(32): p. 1430214310.

131. Arrigo, R., et al., Dynamic surface rearrangement and thermal stability of nitrogen functional groups on carbon nanotubes. Chemical Communications, 2008(40): p. 4891-4893.

132. Deng, S., et al., A glucose biosensor based on direct electrochemistry of glucose oxidase immobilized on nitrogen-doped carbon nanotubes. Biosensors and Bioelectronics, 2009. 25(2): p. 373-377.

133. Sharma, K. and M. Shukla, Molecular modeling of the mechanical behavior of carbon fiber-amine functionalized multiwall carbon nanotube/epoxy composites. New Carbon Materials, 2014. 29(2): p. 132-142.

134. Donaldson, K. and C.A. Poland, Nanotoxicity: challenging the myth of nano-specific toxicity. Current opinion in biotechnology, 2013. 24(4): p. 724-734.

135. Ray, P.D., B.-W. Huang, and Y. Tsuji, Reactive oxygen species (ROS) homeostasis and redox regulation in cellular signaling. Cellular signalling, 2012. 24(5): p. 981-990.

136. Belousov, V., G. Enikolopov, and N. Mishina, Compartmentalization of ROS-mediated signal transduction. Russian Journal of Bioorganic Chemistry, 2013. 39(4): p. 341-355.

137. Boonstra, J. and J.A. Post, Molecular events associated with reactive oxygen species and cell cycle progression in mammalian cells. Gene, 2004. 337: p. 1-13. 
138. Verbon, E.H., J.A. Post, and J. Boonstra, The influence of reactive oxygen species on cell cycle progression in mammalian cells. Gene, 2012. 511(1): p. 1-6.

139. Pacurari, M., et al., Cell permeability, migration, and reactive oxygen species induced by multiwalled carbon nanotubes in human microvascular endothelial cells. Journal of Toxicology and Environmental Health, Part A, 2012. 75(2): p. 112-128.

140. Ye, S.-F., et al., ROS and NF-KB are involved in upregulation of IL-8 in A549 cells exposed to multiwalled carbon nanotubes. Biochemical and biophysical research communications, 2009. 379(2): p. $643-648$.

141. Rawlings, J.S., K.M. Rosler, and D.A. Harrison, The JAK/STAT signaling pathway. Journal of cell science, 2004. 117(8): p. 1281-1283.

142. Hirano, S., et al., Uptake and cytotoxic effects of multi-walled carbon nanotubes in human bronchial epithelial cells. Toxicology and applied pharmacology, 2010. 249(1): p. 8-15.

143. Pacurari, M., et al., Oxidative and molecular interactions of multi-wall carbon nanotubes (MWCNT) in normal and malignant human mesothelial cells. Nanotoxicology, 2008. 2(3): p. 155170.

144. Wynn, T.A., Integrating mechanisms of pulmonary fibrosis. The Journal of experimental medicine, 2011. 208(7): p. 1339-1350.

145. McAnulty, R.J., Fibroblasts and myofibroblasts: their source, function and role in disease. The international journal of biochemistry \& cell biology, 2007. 39(4): p. 666-671.

146. Selman, M. and A. Pardo, Idiopathic pulmonary fibrosis: an epithelial/fibroblastic cross-talk disorder. Respiratory research, 2001. 3(1): p. 1.

147. Lynch, J.P., Idiopathic pulmonary fibrosis. Vol. 185;185.;. 2004, New York: Marcel Dekker.

148. Todd, N.W., I.G. Luzina, and S.P. Atamas, Molecular and cellular mechanisms of pulmonary fibrosis. Fibrogenesis Tissue Repair, 2012. 5(1): p. 11. 
149. Wang, P., et al., Multiwall Carbon Nanotubes Directly Promote Fibroblast-Myofibroblast and Epithelial-Mesenchymal Transitions through the Activation of the TGF-6/Smad Signaling Pathway. Small, 2015. 11(4): p. 446-455.

150. Vietti, G., et al., Towards predicting the lung fibrogenic activity of nanomaterials: experimental validation of an in vitro fibroblast proliferation assay. Particle and Fibre Toxicology, 2013. 10: p. $52-52$.

151. Manke, A., L. Wang, and Y. Rojanasakul, Pulmonary toxicity and fibrogenic response of carbon nanotubes. Toxicology mechanisms and methods, 2013. 23(3): p. 196-206.

152. Gasser, M., et al., Pulmonary surfactant coating of multi-walled carbon nanotubes (MWCNTs) influences their oxidative and pro-inflammatory potential in vitro. Particle and Fibre Toxicology, 2012. 9(1): p. 1-13.

153. Zhao, Y., B.L. Allen, and A. Star, Enzymatic degradation of multiwalled carbon nanotubes. The journal of physical chemistry. A, 2011. 115(34): p. 9536-9544.

154. Sakai, N. and A.M. Tager, Fibrosis of two: epithelial cell-fibroblast interactions in pulmonary fibrosis. Biochimica et Biophysica Acta (BBA)-Molecular Basis of Disease, 2013. 1832(7): p. 911921.

155. Stumm, C.L., et al., Lung remodeling in a mouse model of asthma involves a balance between TGF-61 and BMP-7. PloS one, 2014. 9(4): p. e95959.

156. Kobayashi, T., et al., Bidirectional role of IL-6 signal in pathogenesis of lung fibrosis. Respiratory research, 2015. 16(1): p. 99.

157. Car, B.D., et al., ELEVATED IL-8 AND MCP-1 IN THE BRONCHOALVEOLAR LAVAGE FLUID OF PATIENTS WITH IDIOPATHIC PULMONARY FIBROSIS AND PULMONARY SARCOIDOSIS. AMERICAN JOURNAL OF RESPIRATORY AND CRITICAL CARE MEDICINE, 1994. 149(3): p. 655-659. 
158. Takizawa, H., et al., Increased IL-6 and IL-8 in bronchoalveolar lavage fluids (BALF) from patients with sarcoidosis: correlation with the clinical parameters. Clinical \& Experimental Immunology, 1997. 107(1): p. 175-181.

159. Mutsaers, S.E., IL-6 FAMILY PROTEINS IN THE REGULATION OF PULMONARY FIBROSIS. WOUND REPAIR AND REGENERATION, 2010. 18(4): p. A62-A62.

160. Dockery, D.W. and C.A. Pope, Acute respiratory effects of particulate air pollution. Annual review of public health, 1994. 15(1): p. 107-132.

161. Polk, W.W., et al., Aerosol generation and characterization of multi-walled carbon nanotubes exposed to cells cultured at the air-liquid interface. Particle and Fibre Toxicology, 2015. 13: p. 20.

162. Briand, J.-P., et al., Cellular uptake of functionalized carbon nanotubes is independent of functional group and cell type. Nature Nanotechnology, 2007. 2(2): p. 108-113.

163. Zhang, X., et al., Tuning the cellular uptake and cytotoxicity of carbon nanotubes by surface hydroxylation. Journal of Nanoparticle Research, 2011. 13(12): p. 6941-6952.

164. Wang, X., et al., Quantitative techniques for assessing and controlling the dispersion and biological effects of multiwalled carbon nanotubes in mammalian tissue culture cells. ACS nano, 2010. 4(12): p. 7241-7252.

165. Petersen, E.J., Q. Huang, and J.W.J. Weber, Relevance of octanol-water distribution measurements to the potential ecological uptake of multi-walled carbon nanotubes. Environmental toxicology and chemistry / SETAC, 2010. 29(5): p. 1106-n/a.

166. Guidance on Sample Preparation and Dosimetry for the Safety Testing of Manufactured Nanomaterials, O.f.E.C.-o.a. Development, Editor. 2012.

167. Keane, M.P., et al., The CXC chemokines, IL-8 and IP-10, regulate angiogenic activity in idiopathic pulmonary fibrosis. The Journal of Immunology, 1997. 159(3): p. 1437-1443. 
168. Le, T.T.T., et al., Blockade of IL-6 Trans Signaling Attenuates Pulmonary Fibrosis. JOURNAL OF IMMUNOLOGY, 2014. 193(7): p. 3755-3768.

169. Saito, F., et al., Role of Interleukin-6 in Bleomycin-Induced Lung Inflammatory Changes in Mice. American Journal of Respiratory Cell and Molecular Biology, 2008. 38(5): p. 566-571.

170. Fatkhutdinova, L.M., et al., Fibrosis biomarkers in workers exposed to MWCNTs. Toxicology and Applied Pharmacology, 2016. 299: p. 125-131.

171. Shvedova, A.A., et al., Integrated Analysis of Dysregulated ncRNA and mRNA Expression Profiles in Humans Exposed to Carbon Nanotubes. PloS one 2016. 11(3): p. e0150628.

172. Erdely, A., et al., Cross-Talk between Lung and Systemic Circulation during Carbon Nanotube Respiratory Exposure. Potential Biomarkers. Nano Letters, 2009. 9(1): p. 36-43.

173. Yoshida, M., et al., A histologically distinctive interstitial pneumonia induced by overexpression of the interleukin 6, transforming growth factor beta 1, or platelet-derived growth factor B gene. Proceedings of the National Academy of Sciences of the United States of America, 1995. 92(21): p. $9570-9574$.

174. Shahar, I., et al., Effect of IL-6 on Alveolar Fibroblast Proliferation in Interstitial Lung Diseases. Clinical Immunology and Immunopathology, 1996. 79(3): p. 244-251.

175. Rose-John, S., et al., Structural and functional studies on the human interleukin-6 receptor. Binding, cross-linking, internalization, and degradation of interleukin- 6 by fibroblasts transfected with human interleukin-6-receptor cDNA. Journal of Biological Chemistry, 1991. 266(6): p. 38413846.

176. Olman, M.A., et al., Pulmonary edema fluid from patients with early lung injury stimulates fibroblast proliferation through IL-16-induced IL-6 expression. The Journal of Immunology, 2004. 172(4): p. 2668-2677. 
177. Fries, K.M., M.E. Felch, and R.P. Phipps, Interleukin-6 is an autocrine growth factor for murine lung fibroblast subsets. American Journal of Respiratory Cell and Molecular Biology, 1994. 11(5): p. 552.

178. Maiorano, G., et al., Effects of Cell Culture Media on the Dynamic Formation of Protein-Nanoparticle Complexes and Influence on the Cellular Response. ACS Nano, 2010. 4(12): p. 7481-7491.

179. Burch, H.J., et al., Doping of carbon nanotubes with nitrogen improves protein coverage whilst retaining correct conformation. Nanotechnology, 2008. 19(38): p. 384001.

180. Misra, S.K., et al., The complexity of nanoparticle dissolution and its importance in nanotoxicological studies. Science of The Total Environment, 2012. 438: p. 225-232.

181. Gardner, D.E., Toxicology of the lung. 2006, Boca Raton, FL: CRC/Taylor \& Francis.

182. Riddick, T.M., Control of colloid stability through zeta potential. Blood, 1968. 10(1).

183. Fenoglio, I., et al., Reactivity of carbon nanotubes: free radical generation or scavenging activity? Free Radical Biology and Medicine, 2006. 40(7): p. 1227-1233.

184. Mitchell, L.A., et al., Pulmonary and systemic immune response to inhaled multiwalled carbon nanotubes. Toxicol Sci, 2007. 100(1): p. 203-14.

185. Manke, A., S. Luanpitpong, and Y. Rojanasakul, Potential Occupational Risks Associated with Pulmonary Toxicity of Carbon Nanotubes. Occupational medicine \& health affairs, 2014. 2: p. 1000165.

186. Yu, M., et al., MWCNTs Induce ROS Generation, ERK Phosphorylation, and SOD-2 Expression in Human Mesothelial Cells. Int J Toxicol, 2016. 35(1): p. 17-26.

187. Jiang, Y., et al., Modulation of apoptotic pathways of macrophages by surface-functionalized multi-walled carbon nanotubes. PLoS One, 2013. 8(6): p. e65756. 
188. Vietti, G., et al., Towards predicting the lung fibrogenic activity of MWCNT: Key role of endocytosis, kinase receptors and ERK 1/2 signaling. Nanotoxicology, 2015: p. 1-13.

189. Dong, J. and Q. Ma, In vivo activation of a Thelper 2-driven innate immune response in lung fibrosis induced by multi-walled carbon nanotubes. Archives of Toxicology, 2016: p. 1-18.

190. Mangum, J.B., et al., Co-culture of primary pulmonary cells to model alveolar injury and translocation of proteins. In vitro cellular \& developmental biology, 1990. 26(12): p. 1135-1143.

191. Prasad, R.Y., et al., Cellular interactions and biological responses to titanium dioxide nanoparticles in HepG2 and BEAS-2B cells: Role of cell culture media. Environmental and Molecular Mutagenesis, 2014. 55(4): p. 336-342.

192. Ryman-Rasmussen, J.P., et al., Inhaled Multiwalled Carbon Nanotubes Potentiate Airway Fibrosis in Murine Allergic Asthma. American Journal of Respiratory Cell and Molecular Biology, 2009. 40(3): p. 349-358.

193. Bonner, J.C., Regulation of PDGF and its receptors in fibrotic diseases. Cytokine \& growth factor reviews, 2004. 15(4): p. 255-273.

194. Ingram, J.L., et al., IL-13 and IL-16 promote lung fibroblast growth through coordinated upregulation of PDGF-AA and PDGF-R $\alpha$. The FASEB journal, 2004. 18(10): p. 1132-1134.

195. Knight, D.A., et al., The role of gp130/IL-6 cytokines in the development of pulmonary fibrosis: critical determinants of disease susceptibility and progression? Pharmacology and Therapeutics, 2003. 99(3): p. 327-338.

196. Liu, X., et al., The CC Chemokine Ligand 2 (CCL2) Mediates Fibroblast Survival through IL-6. American Journal of Respiratory Cell and Molecular Biology, 2007. 37(1): p. 121-128.

197. Moodley, Y.P., et al., Inverse effects of interleukin-6 on apoptosis of fibroblasts from pulmonary fibrosis and normal lungs. American journal of respiratory cell and molecular biology, 2003. 29(4): p. 490-498. 
198. Moodley, Y.P., et al., Fibroblasts Isolated from Normal Lungs and Those with Idiopathic Pulmonary Fibrosis Differ in Interleukin-6/gp130-Mediated Cell Signaling and Proliferation. The American Journal of Pathology, 2003. 163(1): p. 345-354.

199. Deshmane, S.L., et al., Monocyte chemoattractant protein-1 (MCP-1): an overview. J Interferon Cytokine Res, 2009. 29(6): p. 313-26.

200. Shinoda, H., et al., Elevated CC chemokine level in bronchoalveolar lavage fluid is predictive of a poor outcome of idiopathic pulmonary fibrosis. Respiration, 2009. 78(3): p. 285-92.

201. Mercer, P.F., et al., Pulmonary Epithelium Is a Prominent Source of Proteinase-activated Receptor-1-inducible CCL2 in Pulmonary Fibrosis. American Journal of Respiratory and Critical Care Medicine, 2009. 179(5): p. 414-425.

202. Okuma, T., et al., C-C chemokine receptor 2 (CCR2) deficiency improves bleomycin-induced pulmonary fibrosis by attenuation of both macrophage infiltration and production of macrophage-derived matrix metalloproteinases. J Pathol, 2004. 204(5): p. 594-604.

203. Agostini, C. and C. Gurrieri, Chemokine/Cytokine Cocktail in Idiopathic Pulmonary Fibrosis. Proceedings of the American Thoracic Society, 2006. 3(4): p. 357-363.

204. Schulz, C., et al., Differences in LPS-Induced Activation of Bronchial Epithelial Cells (BEAS-2B) and Type II-Like Pneumocytes (A-549). Scandinavian Journal of Immunology, 2002. 56(3): p. 294-302.

205. Veranth, J.M., et al., Cytokine responses of human lung cells (BEAS-2B) treated with micron-sized and nanoparticles of metal oxides compared to soil dusts. Particle and fibre toxicology, 2007. 4(1): p. 1.

206. Park, E.-J., et al., Oxidative stress induced by cerium oxide nanoparticles in cultured BEAS-2B cells. Toxicology, 2008. 245(1): p. 90-100. 
207. Xia, T., et al., Interlaboratory Evaluation of in Vitro Cytotoxicity and Inflammatory Responses to Engineered Nanomaterials: The NIEHS Nano GO Consortium. ENVIRONMENTAL HEALTH PERSPECTIVES, 2013. 121(6): p. 683-690. 


\section{Amy L. Mihalchik}

amihalch@mix.wvu.edu

412-480-0853

\section{OBJECTIVE}

$\mathrm{PhD}$ candidate with experience in inhalation toxicology and nanotoxicology seeking a position in toxicology.

\section{EDUCATION}

West Virginia University (PhD) - Morgantown, WV

August 2011 - ongoing

- Doctor of Philosophy in Pharmaceutical and Pharmacological Sciences

- Completed coursework in cancer cell biology, cancer therapeutics, environmental health, scientific writing, nanoscience, and pharmaceutical science

- Field: Nanotoxicology

- Advisor: Dr. Yong Qian

Washington \& Jefferson College (BA) - Washington, PA

August 2007 - May 2011

- Bachelor of Arts in General Biology

- Minor in English

\section{RESEARCH EXPERIENCE}

National Institute for Occupational Safety and Health, Morgantown, WV - Guest Researcher/Worksite Student

Dissertation: The effects of pristine and functionalized multi-walled carbon nanotubes on lung epithelial and fibroblast cells

2012 - present

- Studied effects of pristine multi-walled carbon nanotubes (MWCNT) and nitrogen-doped MWCNT

(ND-MWCNT) at occupationally relevant doses on Human Lung Small Airway Epithelial Cells.

- Carried out pioneering research on the impact of MWCNT physicochemical properties on the pulmonary system in a public health molecular toxicology lab

- Provided an interface for translational interdisciplinary research between government and academic biomedical scientists and material scientists to address scientific issues surrounding MWCNT safety.

- Determined that MWCNT and ND-MWCNT affect Reactive Oxygen Species production, cell cycle progression, and cell signaling processes in lung cells.

- Studied effects of pristine MWCNT and ND-MWCNT on human bronchial epithelial cells and normal lung fibroblasts.

- Investigated mechanisms of MWCNT-induced pulmonary fibrosis in in vitro system utilizing lung epithelial and fibroblast cells.

- Determined that MWCNT and ND-MWCNT induce dose and particle-dependent proinflammatory responses in epithelial cells that may impact fibroblast signaling and proliferation.

- Experienced in utilizing and developing in vitro co-culture systems.

- Used lung epithelial-endothelial co-culture to address toxicity of pristine MWCNT

- Developed preliminary lung epithelial-fibroblast culture system to study mechanism of MWCNT and ND-MWCNT-induced pulmonary fibrosis

- Also utilized various other techniques including Western Blotting, Immunoprecipitation, Confocal Microscopy, ELISA, Flow Cytometry, Cell Cycle Analysis, Cell Proliferation Analysis, RNA

Extraction, and qRT-PCR.

West Virginia University, Morgantown, WV - Doctoral Research Rotations 
Research rotations in the laboratories of Dr. Bingyun Li, Dr. Steven Frisch, and Dr. Letha Sooter

- Assisted on a project assessing the toxicity and potential therapeutic uses of nanoparticles in the Department of Orthopedics.

- Became proficient in cell culture and basic molecular biology techniques through a breast cancerfocused research project.

- Assisted on a project to identify specific molecular recognition elements (MREs) to detect malignant prostate cancer cells using systematic evolution of ligands by exponential enrichment (SELEX).

\section{AWARDS \& GRANTS}

- Society of Toxicology Student Travel Award March 2015

- Integrated Graduate Education and Research Training (IGERT) Fellow August 2014 - Present

- WV NANOSafe Graduate Fellow August 2013- August 2014

\section{PUBLICATIONS}

\section{Conference Publications:}

Mihalchik, A.L. (2015). Effects of Pristine and Nitrogen-Doped Multiwalled Carbon Nanotubes (NDMWCNT) on Reactive Oxygen Species (ROS) and Cell Cycle Progression. In The Toxicologist, abstract of work presented at the Society of Toxicology 54th Annual Meeting, San Diego, CA, March 23-26. pp. 267.

Mihalchik A.L., Porter, D., Castranova, V., Tsuruoka, S., Endo, M., and Qian, Y. (2014).

Effects of MWCNT and Nitrogen-Doped MWCNT in Lung Epithelial Cells. In The Toxicologist, abstract of work presented at the Society of Toxicology 53rd Annual Meeting, Phoenix, AZ, March 23-27. pp. 185.

\section{Publications:}

Mihalchik, A.L., Ding, W., Porter, D., McLoughlin, C., Schwegler-Berry, D., Sisler, J.D., Stefaniak, A., SnyderTalkington, B.N., Cruz-Silva, R., Terrones, M., Tsuruoka, S., Endo, M., Castranova, V., and Qian, Y. (2015). Effects of nitrogen-doped multi-walled carbon nanotubes compared to pristine multi-walled carbon nanotubes on human small airway epithelial cells. Toxicology, 333: 25-36.

\section{CONFERENCES \& PRESENTATIONS}

Mihalchik, A.L., (2015, October) Addressing toxicity: in vitro caveats to the understanding of nanoparticle research and human health. Oral talk presented at the REN@WVU-NEEP Symposium, Morgantown, WV.

Mihalchik, A.L., (2015, April) Nanoparticles: it's the little things in life that count. Oral talk presented at Davis \& Elkins College in Elkins, WV.

Mihalchik, A.L., Ding, W., Porter, D., McLoughlin, C., Schwegler-Berry, D., Sisler, J.D., Stefaniak, A., SnyderTalkington, B.N., Cruz-Silva, R., Terrones, M., Tsuruoka, S., Endo, M., Castranova, V., and Qian, Y. (2015, March) Effects of Pristine and Nitrogen-Doped Multiwalled Carbon Nanotubes (ND-MWCNT) on Reactive Oxygen Species (ROS) and Cell Cycle Progression. Poster session presented at the Society of Toxicology 54th Annual Meeting, San Diego, CA.

Mihalchik A.L., Ding, W., Porter, D., McLoughlin, C., Schwegler-Berry, D., Sisler, J.D., Stefaniak, A., SnyderTalkington, B.N., Cruz-Silva, R., Terrones, M., Tsuruoka, S., Endo, M., Castranova, V., and Qian, Y. (2015, February) Effects of nitrogen-doped multi-walled carbon nanotubes compared to pristine multi-walled carbon nanotubes on human small airway epithelial cells. Poster session presented at the West Virginia University E.J. Van Liere Memorial Convocation \& HSC Research Day, Morgantown, WV. 
Mihalchik A.L., McLoughlin, C., Schwegler-Berry, D., Farcas M., Shvedova, A., Porter, D., Tsuruoka, S., Endo, M., Castranova, V., and Qian, Y. (2014, June) Nitrogen-doped Multi-walled Carbon Nanotube-induced Effects in Human Small Airway Epithelial Cells. Poster session presented at the West Virginia University Bench to Bedside: Translational Pharmacy Meeting, Morgantown, WV.

Mihalchik A.L. McLoughlin, C., Schwegler-Berry, D., Farcas M., Shvedova, A., Porter, D., Tsuruoka, S., Endo, M., Castranova, V., and Qian, Y. (2014, July) Nitrogen-doped Multi-walled Carbon Nanotube-induced Effects in Human Small Airway Epithelial Cells. Poster session presented at the NIOSH Intramural Science Meeting, Morgantown, WV.

\section{PROFESSIONAL AFFILIATIONS}

- Society of Toxicology Member

- Allegheny-Erie Society of Toxicology Chapter Member

- American Association of Pharmaceutical Scientists Member

- Member of Rho Chi Academic Honor Society in Pharmacy Member

\section{LEADERSHIP \& SERVICE}

- School of Pharmacy Graduate Research Advisory Committee - Student Representative September 2015 - present

- Morgantown Children's Museum Science Days 2014-Present Volunteer

- WVU American Association of Pharmaceutical Scientists Chapter Secretary 2014 - 2015

- Teaching Assistant in undergraduate Immunology and Microbiology lab courses 2014 - 2015

- West Virginia Science Bowl 2014, 2015 Volunteer

- Morgantown Boys and Girls Club 2013-2014 Volunteer

- Planned and coordinated scientific content

- Developed and actively participated in a public health laboratory outreach day with local high school students

- Led local children in 4-H in nanotechnology outreach activities at summer day camp

\section{REFERENCES}

\begin{tabular}{|l|l|l|l|}
\hline Name & Title & Phone & Email \\
\hline Yong Qian & $\begin{array}{l}\text { Research } \\
\text { Biologist }\end{array}$ & $304-285-6286$ & yaq2@cdc.gov \\
\hline Vince Castranova & Professor & $304-594-1602$ & vcastran@hsc.wvu.edu \\
\hline Kimberly Quedado & $\begin{array}{l}\text { IGERT Grant } \\
\text { Coordinator }\end{array}$ & $304-293-7382$ & kimberly.quedado@mail.wvu.edu \\
\hline
\end{tabular}

